University of Tennessee Health Science Center

UTHSC Digital Commons

\title{
$5-2009$
}

\section{Targeted Delivery of Surface Modified Nanoparticles: Modulation of Inflammation for Acute Lung Injury}

Hari R. Desu

University of Tennessee Health Science Center

Follow this and additional works at: https://dc.uthsc.edu/dissertations

Part of the Pharmacy and Pharmaceutical Sciences Commons

\section{Recommended Citation}

Desu, Hari R. , "Targeted Delivery of Surface Modified Nanoparticles: Modulation of Inflammation for Acute Lung Injury" (2009). Theses and Dissertations (ETD). Paper 54. http://dx.doi.org/10.21007/ etd.cghs.2009.0066.

This Dissertation is brought to you for free and open access by the College of Graduate Health Sciences at UTHSC Digital Commons. It has been accepted for inclusion in Theses and Dissertations (ETD) by an authorized administrator of UTHSC Digital Commons. For more information, please contact jwelch30@uthsc.edu. 


\title{
Targeted Delivery of Surface Modified Nanoparticles: Modulation of Inflammation for Acute Lung Injury
}

\begin{abstract}
The objective of the study is to demonstrate alleviation of pulmonary inflammation associated with acute lung injury (ALI) using novel surface modified nanoparticles. ALI is characterized by three main pathological events: I) pulmonary edema, II) excessive pro-inflammatory cytokines and chemokines production from alveolar epithelial and endothelial cells and III) leukocyte migration from blood circulation into alveoli. Currently, there is no FDA approved pharmacological treatment available except the supportive mechanical ventilation therapy. Numerous clinical trials involving pharmacological therapies aimed at different pathological targets turned unsuccessful. National Institute of Heart Lung and Blood Institute (NHLBI) cited underappreciation of drug delivery systems as one of the attributed drawbacks.
\end{abstract}

During $A L I$, the $\alpha \vee \beta 6 / \alpha \vee \beta 5$ integrins of alveolar epithelium and endothelium, respectively play a critical role in mediating pulmonary edema in interleukin-1b (IL-1b) stimulated ALI models. A transient blockade of both integrins by antibodies (Ab) or small Arg-Gly-Asp (RGD) peptide sequences may provide new causal therapies for pulmonary edema. A transcription factor, nuclear factor-kB (NF-kB) is associated with the regulation of a battery of genes that encode for pro-inflammatory cytokines, chemokines and cell adhesion molecules (CAMs). Glucocorticoids (GCs) exert their anti-inflammatory effects by NF-kB transcriptional interference mechanisms. Besides the above inflammatory mechanisms, leukocyte migration is another hallmark feature responsible for part of ALI pathogenesis. Leukocyte migration is dictated by sequential activation of adhesion molecules and their ligands on both leukocytes and endothelial cells (ECs). Intercellular adhesion molecule-1 (ICAM-1) located on alveolar endothelium plays a significant role in the recruitment of leukocytes into alveoli. Blockade of ICAM-1 receptors using antiICAM-1 antibodies may impede leukocyte migration into alveoli.

In view of high mortality associated with ALI, there is an urgent need for a drug delivery system that inhibits majority of pathological events. In drug targeting research, liposomes are considered as versatile for their ability to carry drug payloads and flexible enough to modulate their surface for targeting purposes. In the current investigation, I developed surface modified stable liposome formulation for delivery of methylprednisolone succinate (a glucocorticoid; MPS) to the lung. Liposomes were targeted to the disease sites through different routes of administration to avail the spatial expression of the receptors in response to IL- $1 \mathrm{~b}$ stimulus. MPS- $\mathrm{L}^{\mathrm{CRGD}}$ targeted to avb6 integrins of alveolar epithelium attenuated pulmonary edema. MPS- $\mathrm{L}^{\mathrm{Ab}}$ were able to modulate neutrophil migration. Moreover, MPS released from targeted liposomes inhibited the expression of pro-inflammatory cytokines and chemokines. The research work demonstrated the concept of using drug encapsulated and surface modified nanoparticles for therapeutic intervention of ALI.

\section{Document Type}

Dissertation

Degree Name

Doctor of Philosophy $(\mathrm{PhD})$

Program

Pharmaceutical Sciences 


\section{Research Advisor}

George C. Wood, Ph.D.

\section{Keywords}

Acute lung injury, drug targeting, liposomes, lyophilization, nanoparticles, peptide

\section{Subject Categories}

Medicine and Health Sciences | Pharmacy and Pharmaceutical Sciences 
Targeted Delivery of Surface Modified Nanoparticles:

Modulation of Inflammation for Acute Lung Injury

\author{
A Dissertation \\ Submitted To \\ The Graduate Studies Council \\ The University of Tennessee \\ Health Science Center
}

In Partial Fulfillment

Of the Requirements for the Degree

Doctor of Philosophy

From The University of Tennessee

By

Hari R. Desu

May 2009 
Copyright (C) 2009 by Hari R. Desu

All rights reserved 


\section{DEDICATION}

This dissertation is dedicated to

My grand parents, parents, brothers and wife

For their endless love and support 


\section{ACKNOWLEDGEMENTS}

I extend my gratitude to many people without whom this dissertation could not be accomplished. First, I thank my advisor, Prof. George C. Wood and Prof. Laura A. Thoma for giving me the opportunity to work in Parenteral Medications Laboratories (PML). I am very grateful for this precious opportunity to work under their professional guidance and consistent encouragement. I would also like to thank the other members of my dissertation committee, for all the advice, assistance and confidence that they have afforded me: Dr. Mostafa W. Gaber, Dr. Ram I. Mahato, Dr. Bob M. Moore, Dr. Atul J. Shukla and Dr. Charles R. Yates.

My gratitude is extended to the fellow members (current and past) of Wood's lab, Dr. Murali K. Divi, Vinayagam and Dr. Himanshu Bhattacharjee, who have helped me in different ways. In particular, I want to thank Dr. Murali K. Divi and Vinayagam for their consistent support. I have been lucky enough to be associated with other PML group members, Frank Horton, Dr. Robert J. Nolly, Barry Braganza, Dr. Charles T. May, Jennifer Hart, Gwendolyn Stornes, Dr. Vivian Loveless and Dr. Hassan Almoazen with whom, I shared pleasant experiences. Many people outside of the lab have provided a lot of assistance and advice, for which I am extremely grateful.

I would like to thank the University of Tennessee Health Science Center and the entire faculty and staff in the Department of Pharmaceutical Sciences for their support during my graduate study. I extend my thanks to Parenteral Drug Association (PDA) for providing predoctoral fellowship. 


\begin{abstract}
The objective of the study is to demonstrate alleviation of pulmonary inflammation associated with acute lung injury (ALI) using novel surface modified nanoparticles. ALI is characterized by three main pathological events: I) pulmonary edema, II) excessive pro-inflammatory cytokines and chemokines production from alveolar epithelial and endothelial cells and III) leukocyte migration from blood circulation into alveoli. Currently, there is no FDA approved pharmacological treatment available except the supportive mechanical ventilation therapy. Numerous clinical trials involving pharmacological therapies aimed at different pathological targets turned unsuccessful. National Institute of Heart Lung and Blood Institute (NHLBI) cited underappreciation of drug delivery systems as one of the attributed drawbacks.

During ALI, the $\alpha \mathrm{v} \beta 6 / \alpha \mathrm{v} \beta 5$ integrins of alveolar epithelium and endothelium, respectively play a critical role in mediating pulmonary edema in interleukin-1 $\beta$ (IL-1 $\beta$ ) stimulated ALI models. A transient blockade of both integrins by antibodies $(\mathrm{Ab})$ or small Arg-Gly-Asp (RGD) peptide sequences may provide new causal therapies for pulmonary edema. A transcription factor, nuclear factor- $\kappa \mathrm{B}(\mathrm{NF}-\kappa \mathrm{B})$ is associated with the regulation of a battery of genes that encode for pro-inflammatory cytokines, chemokines and cell adhesion molecules (CAMs). Glucocorticoids (GCs) exert their anti-inflammatory effects by NF- $\kappa \mathrm{B}$ transcriptional interference mechanisms. Besides the above inflammatory mechanisms, leukocyte migration is another hallmark feature responsible for part of ALI pathogenesis. Leukocyte migration is dictated by sequential activation of adhesion molecules and their ligands on both leukocytes and endothelial cells (ECs). Intercellular adhesion molecule-1 (ICAM-1) located on alveolar endothelium plays a significant role in the recruitment of leukocytes into alveoli. Blockade of ICAM-1 receptors using antiICAM-1 antibodies may impede leukocyte migration into alveoli.
\end{abstract}

In view of high mortality associated with ALI, there is an urgent need for a drug delivery system that inhibits majority of pathological events. In drug targeting research, liposomes are considered as versatile for their ability to carry drug payloads and flexible enough to modulate their surface for targeting purposes. In the current investigation, I developed surface modified stable liposome formulation for delivery of methylprednisolone succinate (a glucocorticoid; MPS) to the lung. Liposomes were targeted to the disease sites through different routes of administration to avail the spatial expression of the receptors in response to IL-1 $\beta$ stimulus. MPS- ${ }^{\text {cRGD }}$ targeted to $\alpha v \beta 6$ integrins of alveolar epithelium attenuated pulmonary edema. MPS- $\mathrm{L}^{\mathrm{Ab}}$ were able to modulate neutrophil migration. Moreover, MPS released from targeted liposomes inhibited the expression of pro-inflammatory cytokines and chemokines. The research work demonstrated the concept of using drug encapsulated and surface modified nanoparticles for therapeutic intervention of ALI. 


\section{TABLE OF CONTENTS}

Chapter 1. Introduction to Targeted Liposomes ................................................................... 1

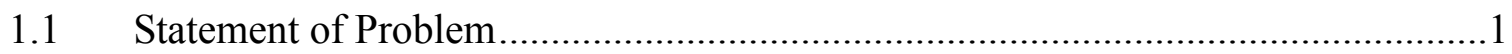

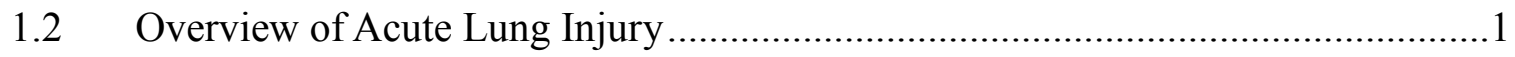

1.2.1 Pulmonary Cytokines and Inflammation in ALI......................................... 3

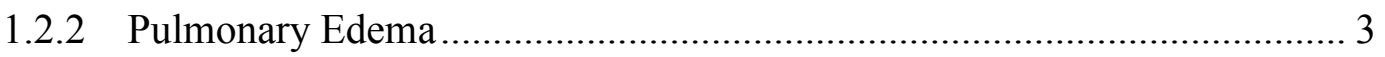

1.2.3 Leukocyte Migration in Pulmonary Vasculature ..................................... 5

1.2.4 Relevance of ALI Models in Therapeutic Intervention ............................... 8

1.2.5 Pharmacological Interventions of ALI in Preclinical and Clinical

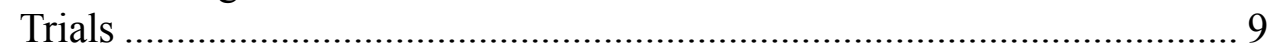

1.2.6 Significance of Drug Delivery in ALI Pathogenesis................................. 12

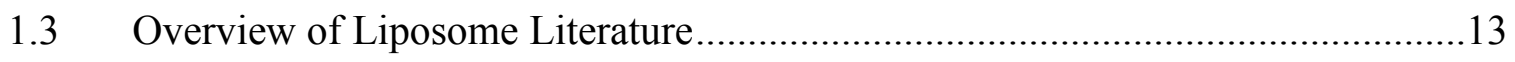

1.3.1 Basic Liposome Composition .............................................................. 14

1.3.2 Physico-chemical Properties of Drugs: Liposome Encapsulation ............. 16

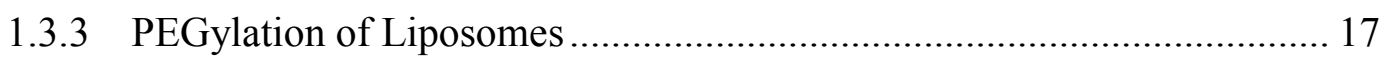

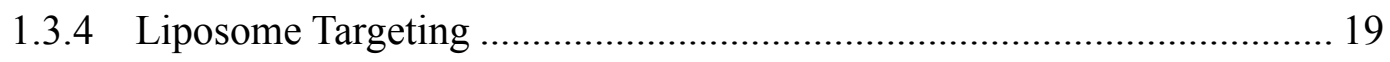

1.3.5 Methods for Attaching Targeting Ligands to Liposomes.......................... 20

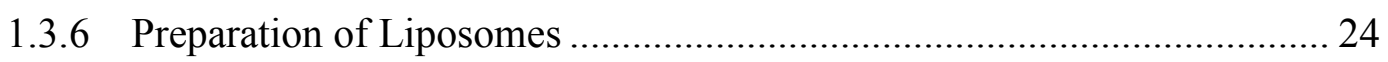

1.3.7 Liposome In Vivo Kinetics: Systemic Administration and

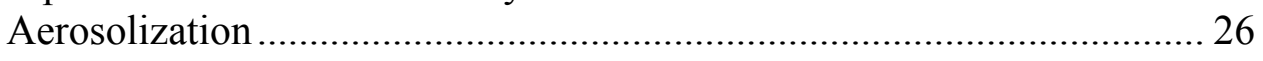

1.3.8 Liposomes: Clinical and Diagnostic Applications .................................... 27

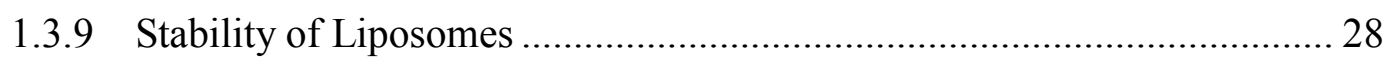

1.3.10 Lyophilization: Stabilization of Liposomes …………………………..... 31

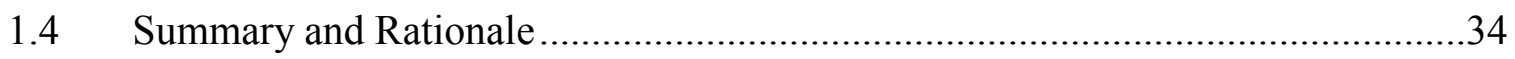

Chapter 2. Central Hypothesis and Specific Aims............................................................... 36

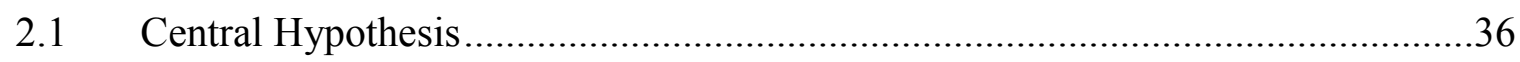

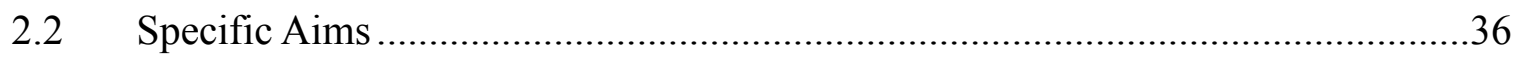

2.2.1 To Develop MPS Encapsulated Targeted Liposomes ................................ 36

2.2.2 To Lyophilize and Develop Stable MPS Encapsulated Targeted

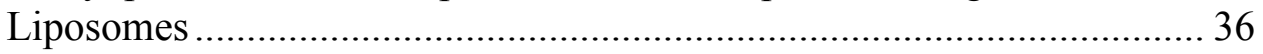

2.2.3 To Modulate Pulmonary Edema via MPS- $\mathrm{L}^{\mathrm{CRGD}}$ Targeting to $\alpha v \beta 6$ Integrins ............................................................................................ 37

2.2.4 To Impede Neutrophil Migration via MPS- ${ }^{\mathrm{Ab}}$ Targeting to ICAM-1

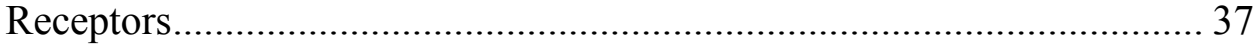




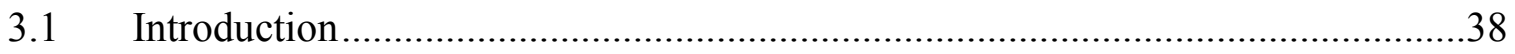

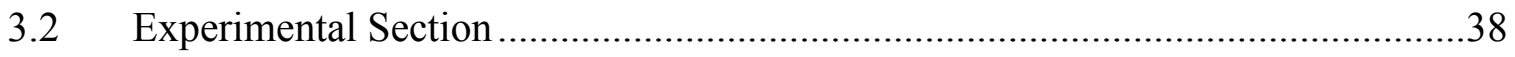

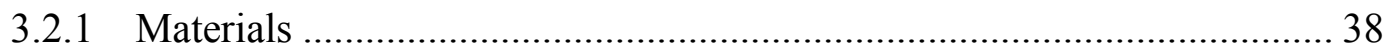

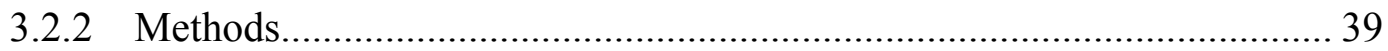

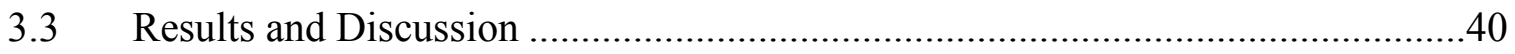

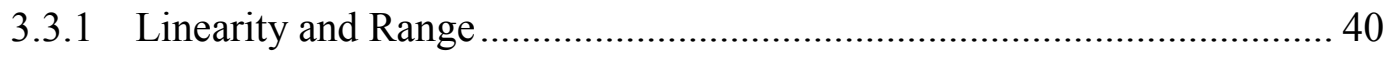

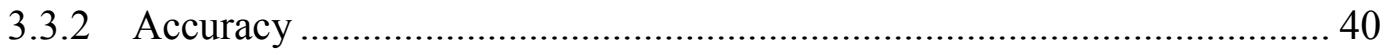

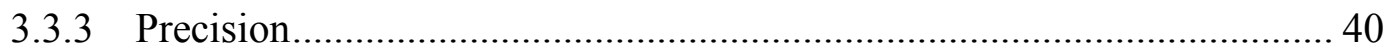

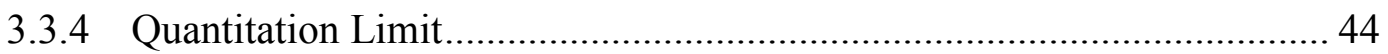

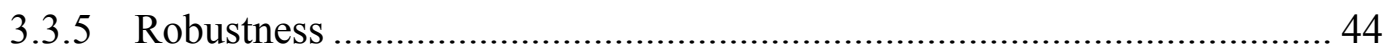

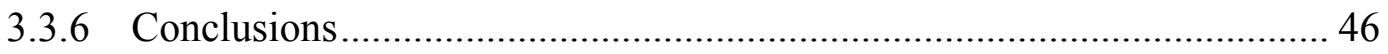

Chapter 4. MPS Encapsulation and Surface Modification of Liposomes .................. 47

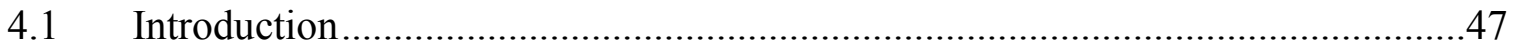

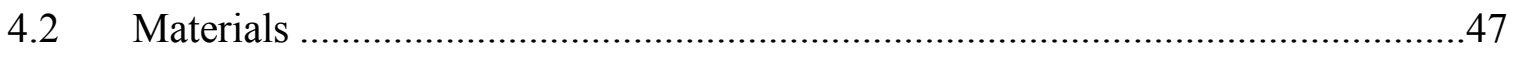

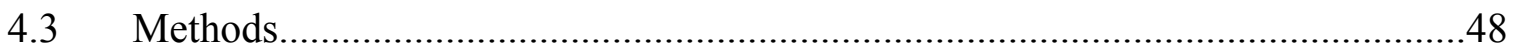

4.3.1 Preparation of MPS-L by Passive Loading (Bangham Method) .............. 48

4.3.2 Active Loading of Amphipathic MPS into Liposomes............................. 48

4.3.3 Synthesis of DSPE-PEG ${ }_{2000}$-maleimide-cRGD Conjugate........................ 49

4.3.4 Insertion of DSPE-PEG ${ }_{2000}$-maleimide-cRGD Conjugate into

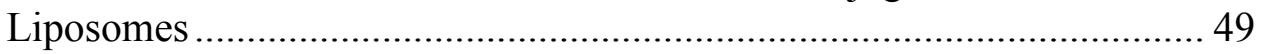

4.3.5 Chemical Characterization of Liposome Dispersion ................................. 51

4.3.6 Physical Characterization of Liposome Dispersion ..................................... 51

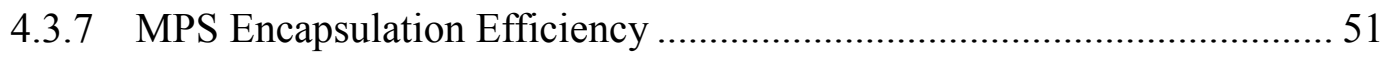

4.3.8 In Vitro Leakage Kinetics of MPS from Liposomes .................................. 53

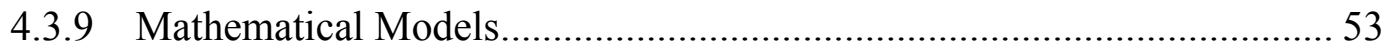

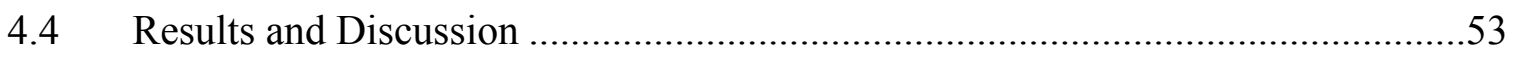

4.4.1 Passive Loading Strategies ............................................................... 53

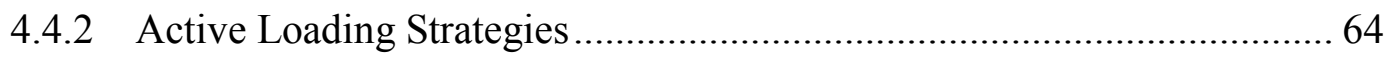

Chapter 5. Protection of Colloidal Properties of MPS- ${ }^{\mathrm{CRGD}}$...................................... 72

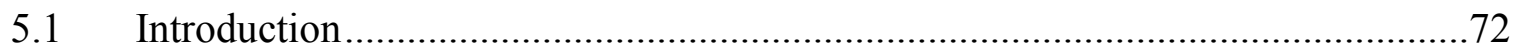

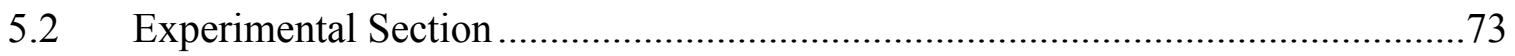

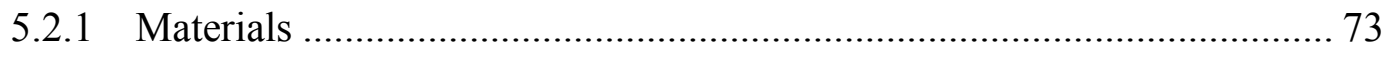

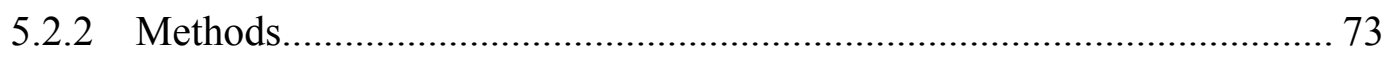

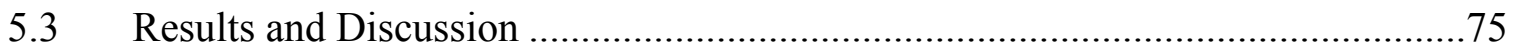


5.3.1 Disaccharides as Lyoprotectants: Evaluation of Appearance of MPS$\mathrm{L}^{\mathrm{cRGD}}$

5.3.2 Effect of Lyoprotectants on Particle Size............................................... 75

5.3.3 Effect of Lyoprotectants on the Thermotropic Phase Behaviour of HSPC before Lyophilization ................................................................ 77

5.3.4 Effect of Lyoprotectants on the Thermotropic Phase Behaviour of HSPC after Lyophilization.............................................................. 80

5.3.5 Amino Acids as Lyoprotectants: Influence of Lyoprotectant Concentration... 82

5.3.6 Influence of Freezing Temperature on the Performance of the

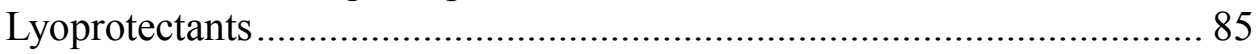

5.3.7 Influence of Lyoprotectants on MPS Leakage from MPS-L ................... 85

Chapter 6. Inhibition of Lung Inflammation Using MPS-L ${ }^{\text {cRGD }}$................................. 89

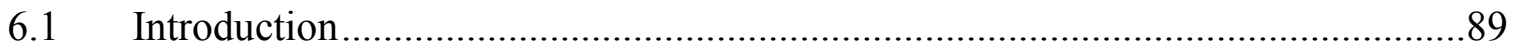

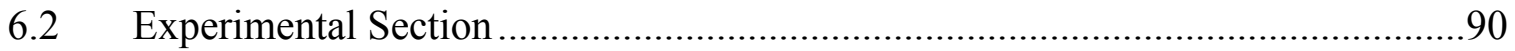

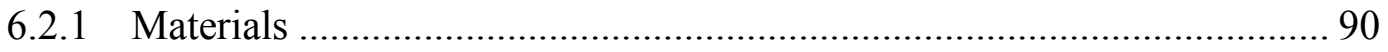

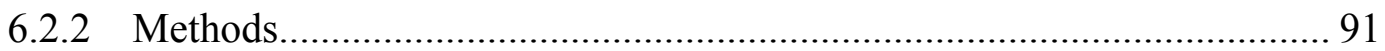

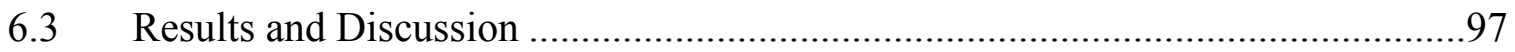

6.3.1 AlphaVBeta6 ( $\alpha$ v $\beta 6$ ) Integrin Receptor Expression in A549 Cells ......... 97

6.3.2 Cell Internalization of MPS-L ${ }^{\text {cRGD }}$ : CLSM Studies .................................. 99

6.3.3 Effect of MPS-L ${ }^{\text {cRGD }}$ on MCP-1 Expression in A549 Cells ..................... 99

6.3.4 Effect of MPS- $\mathrm{L}^{\text {cRGD }}$ on Monocytes Adhesion to IL-1 $\beta$ Stimulated A549 Monolayers........................................................................ 103

6.3.5 Effect of MPS-L ${ }^{\text {cRGD }}$ on ICAM-1 Expression in A549 Cells ................. 103

6.3.6 Effect of MPS-L ${ }^{\text {cRGD }}$ on Neutrophil Adhesion to A549 Monolayers...... 105

6.3.7 Effect of MPS-L ${ }^{\text {cRGD }}$ on Epithelial Permeability in IL-1 $\beta$ Induced A549 Cells ................................................................................... 105

6.3.8 MPS-L ${ }^{\text {cRGD }}$ Inhibits NF- $\kappa$ B Nuclear Translocation in A549 Cells ......... 108

6.3.9 Effect of MPS-L ${ }^{\mathrm{cRGD}}$ on BALF Protein Concentration in IL-1 $\beta$ Induced Rats............................................................................... 111

6.3.10 Effect of MPS-L ${ }^{\text {cRGD }}$ on MCP-1 Production in BALF of IL-1 $\beta$ Induced Rats................................................................................... 111

6.3.11 Effect of MPS-L ${ }^{\text {cRGD }}$ on TNF- $\alpha$ Production in BALF of IL-1 $\beta$

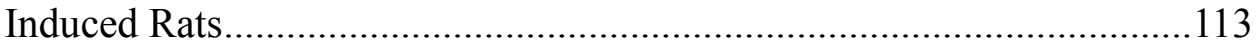

6.3.12 Effect of MPS-L ${ }^{\text {cRGD }}$ on Neutrophil Migration of IL-1 $\beta$ Induced Rats ...113

6.3.13 Effect of MPS-L ${ }^{\text {cRGD }}$ on Neutrophil Accumulation in Lung Parenchyma. 
Chapter 7. Inhibition of Lung Inflammation Using MPS-L ${ }^{\mathrm{Ab}}$

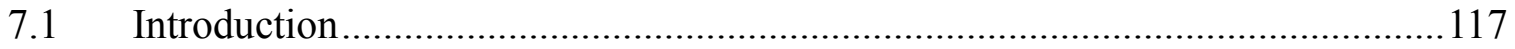

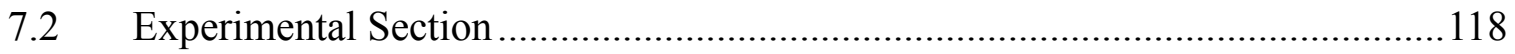

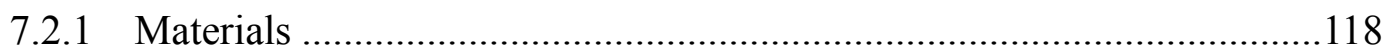

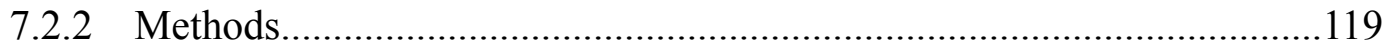

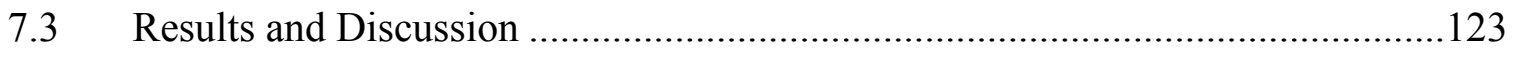

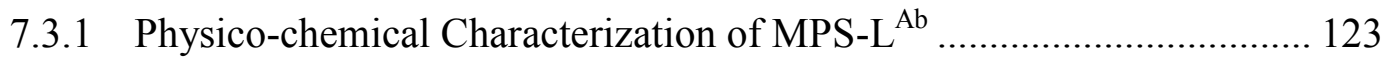

7.3.2 Effect of MPS-L ${ }^{\mathrm{Ab}}$ on BALF Protein Concentration in IL-1 $\beta$ Induced Rats ...................................................................................... 123

7.3.3 Effect of MPS-L ${ }^{\mathrm{Ab}}$ on MCP-1 Production in BALF of IL-1 $\beta$ Induced Rats ....................................................................................... 125

7.3.4 Effect of MPS-L ${ }^{\mathrm{Ab}}$ on TNF- $\alpha$ Production in BALF of IL-1 $\beta$ Induced

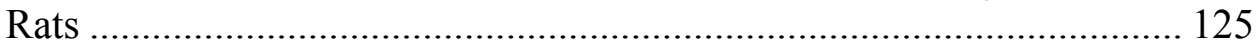

7.3.5 Effect of MPS- $\mathrm{L}^{\mathrm{Ab}}$ on Neutrophil Migration of IL-1 $\beta$ Induced Rats ..... 125

7.3.6 Effect of MPS-L ${ }^{\mathrm{Ab}}$ on Neutrophil Accumulation in Lung Parenchyma . 125

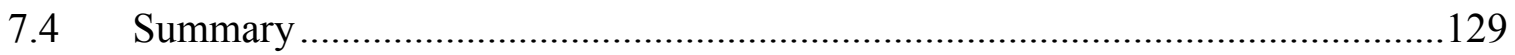

Chapter 8. Summary and Conclusions ................................................................... 130

List of References .................................................................................................... 132

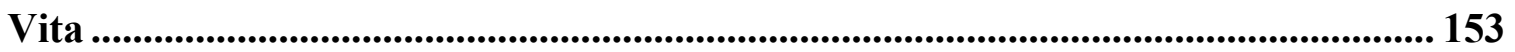




\section{LIST OF TABLES}

Table 1.1 Pharmacological agents in preclinical and clinical phases of drug

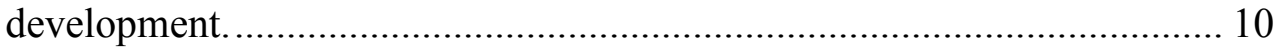

Table 1.2 Liposome products in clinical and preclinical studies............................... 15

Table 3.1 Chromatographic performance data of the method. ................................... 41

Table 3.2 Summary of linear regression data for calibration standards. .................... 42

Table 3.3 Summary of method accuracy results.................................................... 43

Table 3.4 Summary of method precision results. ................................................. 43

Table 3.5 Summary of robustness of the method. ……………............................... 45

Table 4.1 Parameters in the K-P equation for MPS release kinetics from MPS-L..... 60

Table 4.2 Values of $\mathrm{k}$ from the $\mathrm{t}^{0.7}$ law for MPS-L at different temperatures. ........... 63

Table 5.1 The effect of lyoprotectant concentration on the mean diameter of empty liposomes (after lyophilization)..................................................... 84

Table 5.2 The effect of lyophilization protocols on the mean diameter of

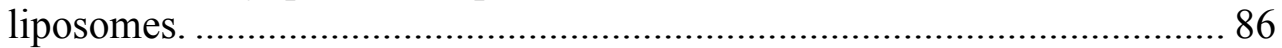

Table 5.3 The effect of lyoprotectant concentration of the mean diameter of

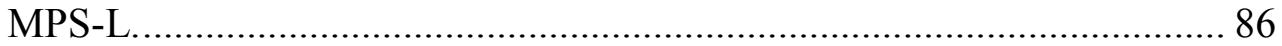

Table 6.1 Primer sequences for $\alpha \mathrm{v}, \beta 6$, MCP-1, ICAM-1 and $\beta$-actin. ..................... 93

Table 6.2 Physico-chemical properties of MPS- ${ }^{\text {CRGD }}$ used in in vitro and in vivo

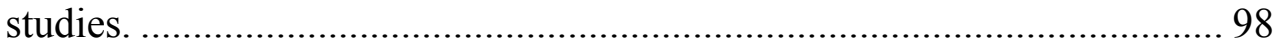

Table 7.1 Physico-chemical properties of MPS-L ${ }^{\mathrm{Ab}}$ used in in vivo studies............ 124 


\section{LIST OF FIGURES}

Figure 1.1 Schematic representation of the normal alveolus and the injured alveolus in the acute phase of ALI/ARDS.

Figure 1.2 Schematic illustration of the effect of interleukin-1 $\beta$ on the alveolar capillary barrier.

Figure 1.3 Schematic diagram of neutrophil and endothelial cell interactions in the pulmonary vasculature.

Figure 1.4 Schematic representation of various classes of liposomes. ...................... 18

Figure 1.5 Schematic representations of different coupling methods used for surface modification of liposomes.

Figure 1.6 Schematic representation of the liposomal loading of weak bases (A) and weak acids (B).

Figure 1.7 Schematic diagram of the Gouy-Chapman-Stern diffuse double layer theory.

Figure 1.8 Schematic representation of different stages of a lyophilization cycle. .... 32

Figure 3.1 Chromatogram of calibration standards of CHOL and HSPC.................. 41

Figure 3.2 Typical chromatogram of linearity of standards containing CHOL and HSPC.

Figure 4.1 Schematic representation of Michael addition reaction between the lipid, DSPE-PEG 2000 -maleimide and cRGD-peptide.

Figure 4.2 Schematic representation of the insertion of the targeting conjugate by post-insertion technique.

Figure 4.3 Schematic representation of the mathematical model based on Rippie's

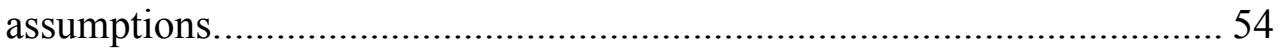

Figure 4.4 Graph depicting the MPS encapsulation efficiency as a function of drug/lipid ratios.

Figure 4.5 Graph depicting the MPS encapsulation efficiency as a function of CHOL fraction.

Figure 4.6 Graph depicting the MPS encapsulation efficiency as a function of PEGylated lipid incorporation.

Figure 4.7 Graph depicting the MPS encapsulation efficiency as a function of number of extrusion cycles.

Figure 4.8 Graph depicting the MPS release from liposome formulations containing different fractions of DSPE-PEG 2000 .

Figure 4.9 Graph depicting the MPS release from PEGylated liposome formulations with mean diameters of 100, 200, 400 and $800 \mathrm{~nm}$.

Figure 4.10 Graph depicting the MPS release rate constant of the liposome formulations with varied mean diameter, calculated from K-P equation. 
Figure 4.11 MPS release rate from PEGylated liposomes stored at different temperatures $4(\mathbf{\square}), 25(\square), 30(\Delta), 37(\boldsymbol{\Delta})$ and $40^{\circ} \mathrm{C}(*)$.

Figure 4.12 Histogram of MPS encapsulation driven by various acetate salt gradients in PEGylated liposomes.

Figure 4.13 Histogram of MPS encapsulation as a function of different ionic strengths of calcium acetate inside the lipoosmes. 66

Figure 4.14 Histogram of MPS encapsulation in liposomes as a function of intravesicular $\mathrm{pH}$.

Figure 4.15 MPS release from liposomes as a function of $\mathrm{pH}$ of the external medium at $25^{\circ} \mathrm{C}$. 68

Figure 4.16 MALDI-MS spectra of the (DSPE-PEG 2000 -maleimide)-cRGD-peptide conjugate.

Figure 5.1 Representative samples of the MPS-L ${ }^{\text {cRGD }}$ lyophilized with high (400 $\mathrm{mM}$; left) and low (50 mM; right) concentrations of trehalose.

Figure 5.2 Transmission electron micrographs ofMPS-L ${ }^{\text {cRGD }}$ before and after lyophilization (i.e. reconstituted) with trehalose.

Figure 5.3 Mean diameter of MPS-L ${ }^{\text {cRGD }}$ as a function of lyoprotectant concentration. 78

Figure 5.4 Thermograms of MPS-L ${ }^{\text {cRGD }}$ containing increasing concentrations of trehalose (A) and sucrose (B) prior to lyophilization.

Figure 5.5 Thermograms of lyophilized, rehydrated MPS- $\mathrm{L}^{\mathrm{cRGD}}$ formulations containing increasing concentrations of sugar.

Figure 5.6 Transmission electron micrographs of MPS-L before (A) and after (B) lyophilization with lysine as external lyoprotectant.

Figure 5.7 Graph depicting the mean diameter of MPS-L as a function of aminoacids as external lyoprotectants used for lyophilization.

Figure 5.8 Graph depicting the MPS leakage from MPS-L, lyophilized with aminoacids as external lyoprotectants.

Figure 6.1 RT-PCR and Western Blot analyses of concentration dependent inhibition of $\alpha v \beta 6$ integrin expression by MPS.

Figure 6.2 Confocal laser fluorescence microscopy of cell internalization of MPS-

L and MPS-L ${ }^{\text {cRGD }}$ in quiescent and IL-1 $\beta$ activated A549 cells.

Figure 6.3 ELISA and RT-PCR analyses of MCP-1 expression in A549 cells as a function of MPS encapsulated formulations.

Figure 6.4 Histogram of the monocyte adhesion as a function of MPS encapsulated formulation treatments in IL-1 $\beta$ activated A549 cells.

Figure 6.5 Densitometric RT-PCR and Western Blot analyses of ICAM-1 expression in A549 cells, treated with MPS-L ${ }^{\text {cRGD }}$.... 106

Figure 6.6 Histogram of neutrophil adhesion as a function of MPS encapsulated formulation treatments in IL-1 $\beta$ activated A549 cells.

Figure 6.7 Histogram of ${ }^{14} \mathrm{C}$-albumin permeability across A549 cells, pretreated with MPS encapsulated formulations and antibodies 
Figure 6.8 EMSA (left panel) of NF- $\kappa \mathrm{B}$ elicitation and Western Bot analysis of $\mathrm{I} \kappa \mathrm{B} \alpha$ and $\mathrm{p}-\mathrm{I} \kappa \mathrm{B} \alpha$ expression pattern in A549 cells, pretreated with MPS-L ${ }^{\mathrm{cRGD}}$ formulation.

Figure 6.9 Histogram depicting the inhibition of BALF protein after nebulization of MPS-L ${ }^{\mathrm{cRGD}}$ in male Sprague-Dawley rats.

Figure 6.10 Histogram of the inhibition of BALF MCP-1 levels after nebulization of MPS-L ${ }^{\text {CRGD }}$ in male Sprague-Dawley rats.

Figure 6.11 Histogram of inhibition of BALF TNF- $\alpha$ levels after nebulization of MPS-L ${ }^{\text {cRGD }}$

Figure 6.12 Histogram of inhibition of BALF levels of neutrophils after nebulization of MPS-L ${ }^{\text {CRGD }}$ in male Sprague-Dawley rats.

Figure 6.13 Histological sections of lung parenchyma after haematoxylin treatment to stain neutrophils.

Figure 7.1 Schematic representation of the coupling of anti-ICAM-1 antibody fragment tethered to the surface of liposomes.

Figure 7.2 SDS-PAGE analysis of DSPE-PEG $2000-\mathrm{mFab}$ ' fragment under nonreducing conditions.

Figure 7.3 Histogram of inhibition of BALF protein in male Sprague-Dawley rats after systemic administration of MPS- $\mathrm{L}^{\mathrm{Ab}}$

Figure 7.4 Histogram of inhibition of BALF MCP-1 levels in male SpragueDawley rats after systemic administration of MPS-L ${ }^{\mathrm{Ab}}$.

Figure 7.5 Histogram of inhibition of BALF TNF- $\alpha$ levels in male SpragueDawley rats after systemic administration of MPS-L ${ }^{\mathrm{Ab}}$.

Figure 7.6 Histogram of inhibition of BALF levels of neutrophils after nebulization of MPS- $\mathrm{L}^{\mathrm{Ab}}$ in male Spague-Dawley rats.

Figure 7.7 Histological sections of lung parenchyma after haematoxylin treatment to stain neutrophils. 


\section{LIST OF ABBREVIATIONS}

$\mathrm{Ab}$

ALI

ANOVA

ARDS

BALF

BDS

BSA

CAM

$\mathrm{ACN}$

CHOL

$\mathrm{Ci}$

CINC-1

CL

CLSM

cRGD-peptide

CTAC

$\mathrm{D}$

Da

DLS

DLVO

DMEM

DOPE

DPPC

DSC

DSPC

DSPE

DTT

DXR

ECs

EDC

EE

ELISA

ELSD

EMSA

EPC

FBS

FCS

FDA
Antibody

Acute lung injury

Analysis of variance

Acute respiratory distress syndrome

Broncho-alveolar lavage fluid

Bonded di-octyl silane

Bovine serum albumin

Cell adhesion molecule

Acetonitrile

Cholesterol

Curie

Cytokine induced neutrophil chemoattractant-1

Cardiolipin

Confocal laser scanning microscopy

Cyclic RGD-peptide

Cetyl triethyl ammonium chloride

Diffusion coefficient

Daltons

Dynamic light scattering

Derjaguin-Landau-Verwey-Overbeek

Dulbecco's modified eagle medium

Dioleoyl phosphoethanolamine

Dipalmitoyl phosphatidylcholine

Differential scanning calorimetry

Distearoyl phosphatidylcholine

Distearoyl phosphatidylethanolamine

Dithiothreitol

Doxorubicin

Endothelial cells

1-ethyl-3-(3-dimethylaminopropyl)-carbodiimide

Encapsulation efficiency

Enzyme linked immunosorbent assay

Evaporative light scattering detection

Electrophoretic mobility shift assay

Egg phosphatidylcholine

Fetal bovine serum

Fetal calf serum

Food and drug administration 
GCs

GM-CSF

ๆ

$\mathrm{H}_{3} \mathrm{PO}_{4}$

HEPES

HP

HPLC

HSPC

i.t.

IACUC

ICAM-1

IL-1b

$\mathrm{J}$

$\lambda$

LAP

$\operatorname{LogD}$

LPS

LTBP-1

MALDI-MS

MCP-1

MIP-1

MLVs

$\mu$

MPO

MPS

MPS-L

MRI

$\mathrm{mV}$

MWCO

NAC

NF-kB

NHS

NIH

NIRF

nm

PA

PBS

PEG

PEG-PE

PG
Glucocorticoids

Granulocyte macrophage colony-stimulating factor

Viscosity

Phosphoric acid

(N-[2-hydroxyethyl]piperazine-N'-[2-ethanesulfonic acid])

Helmholtz plane

High performance liquid chromatography

Hydrogenated soy phosphatidylcholine

Intra-tracheal

Institution Animal Care and Use Committee

Intercellular adhesion molecule-1

Interleukin- $1 \mathrm{~b}$

Joules

Wavelength

latency associated peptide

Partition coefficient

Lipopolysaccharide

latent TGF- $\beta$-binding protein-1

Matrix assisted laser desorption/ionization mass spectrometry

Monocyte chemoattractant protein-1

Macrophage inhibitory protein-1

Multilamellar vesicles

Micrometers

Myeloperoxidase

Methylprednisolone succinate MPS encapsulated liposomes

Magnetic resonance imaging

Milli volts

Molecular weight cut off

$\mathrm{N}$-acetyl cysteine

Nuclear factor-kappa beta

N-hydroxysulfosuccinimide

National institutes of health

Near infrared fluorescence imaging

Nanometers

Phosphatidic acid

Phosphate buffer saline

Polyethylene glycol

PEGylated phosphatidylethanolamine

Phosphatidylglycerol 
Phosphatidylinositol

PL

Ionization constant

PS

Plain liposomes

PSG

Phosphatidylserine

RAD

RES

RGD

RNA

ROS

RPMI

RSD

RT-PCR

$\mathrm{S} / \mathrm{N}$

Penicillin-Streptomycin-Gentamycin

Arginine-alanine-D-aspartic acid

Reticular endothelial system

Arginine-Glycine-D-Aspartic acid

Ribo nucleic acid

Reactive oxygen species

Royal park memorial institute

Relative standard deviation

Reverse transcriptase polymerase chain reaction

SATA

Signal to noise ratio

$\mathrm{SD}$

Succinimidyl-S-acetylthioacetate

SDS-PAGE

SEM

Standard deviation

SOD

SPDP

SUV

TCEP

TEER

TEM

TFA

$\mathrm{Tg}$

$\mathrm{Tg}^{\prime}$

TGF-B

$\mathrm{Tm}$

TNF- $\alpha$

TSA

$\zeta$

Zavg

Sodium dodecyl sulfate-polyacrylamide gel electrophoresis

Standard error of mean

Superoxide dismutase

N-hydroxysuccinimide 3-(2-pyridyldithio)propionate

Small unilamellar vesicles

Tris(2-carboxyethyl)-phosphine $\mathrm{HCl}$

Transendothelial resistance

Transmission electron microscopy

Trifluoro acetic acid

Glass transition temperature

Glass transition temperature of frozen matrix

Transforming growth factor-beta

Phase transition temperature

Tumor necrosis factor-alpha

Total surface area

Zeta potential

Mean diameter 


\section{CHAPTER 1. INTRODUCTION TO TARGETED LIPOSOMES}

\subsection{Statement of Problem}

In 1967, at the Colorado General Hospital, Ashbaugh and colleagues were the first to describe patients with a variety of underlying illnesses characterized by tachypnea, hypoxemia resistant to oxygenation by regular ventilator support, decreased lung compliance, diffuse bilateral infiltrates on chest radiographs and surfactant abnormalities. Together, the above characteristics are defined as acute respiratory distress syndrome (ARDS). ${ }^{1}$ Another term, acute lung injury was used in synonymous with ARDS in most cases, but could only be applied to a broad range of pathological abnormalities in the lung where as ARDS should be reserved for the most severe cases of lung injury. ${ }^{2}$

ALI is a major cause of acute respiratory failure with high morbidity and mortality in critically ill patients. ${ }^{3}$ Recent epidemiologic data indicate that the incidence of ALI defined by consensus physiologic criteria may account for 36,000 deaths per year in U.S.A. ${ }^{4}$ Although, there is evidence that mortality in patients with ALI may have declined over the last 10 to 15 years, it remains high (30-40\%) and is an important cause of pulmonary and non-pulmonary morbidity in ICU patients. ${ }^{5}$ ALI develops in patients of all ages from a variety of clinical disorders. These conditions can be divided into two categories based on whether the insult was direct (pulmonary) or indirect (extrapulmonary). Direct injury can be described as a condition in which the lung epithelium is directly injured. Causes of such lung injury include pneumonia, aspiration of gastric contents, inhalation of toxic substances and pulmonary contusion. ${ }^{6}$ Direct causes of lung injury lead to a syndrome with more predictability and to a short latent period than do indirect causes. ${ }^{7}$ Indirect lung injuries are conditions that activate a systemic inflammatory response. Multiple trauma, burns, sepsis, fat emboli and hemorrhagic shock are all associated with a high risk of developing ALI. So far, mechanical ventilation is the lone supportive therapy for the alleviation of symptoms associated with ALI.

\subsection{Overview of Acute Lung Injury}

The most important pathological finding in the lung during the early stages of ALI is severe pulmonary edema, due to increased permeability of the alveolar endothelial and epithelial barriers. As illustrated in Figure 1.1, the morphological picture has often been labeled as diffuse alveolar damage and includes cellular necrosis, inflammation and fibrosis. Although, pathogenesis of ALI remains unclear, indirect evidence suggests that pulmonary edema occurs after a direct activation due to acute lung inflammation, or as the pulmonary manifestation of a systemic inflammatory response. ${ }^{8}$ The inflammatory response in both cases involves alveolar migration of leukocytes, (e.g. macrophages, monocytes and neutrophils) which are activated by pro-inflammatory cytokines and chemokines. ${ }^{9}$ 


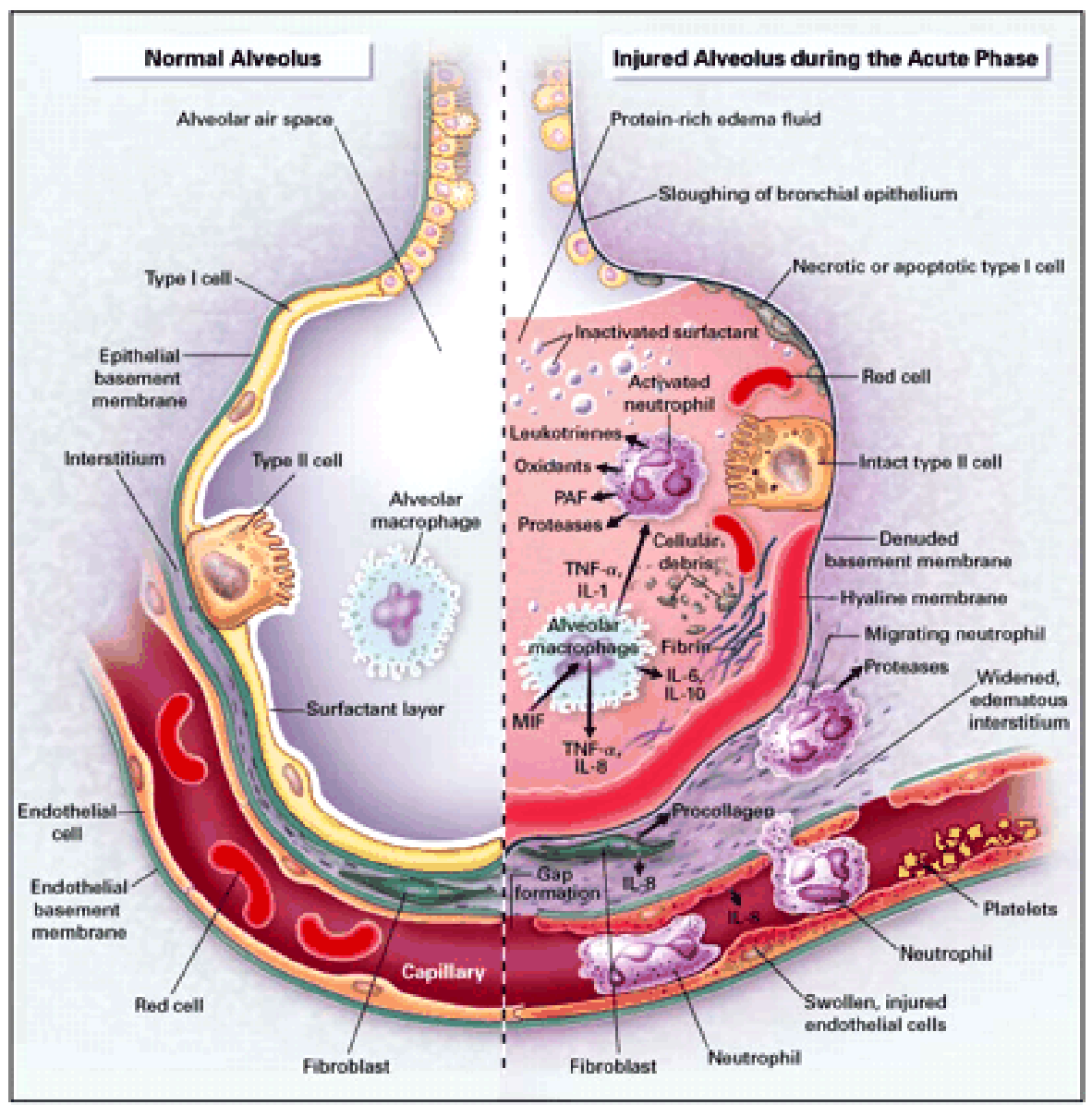

Figure 1.1 Schematic representation of the normal alveolus and the injured alveolus in the acute phase of ALI/ARDS.

In the acute phase of the syndrome (injured alveolus), sloughing of alveolar epithelial cells, with the formation of protein rich hyaline membranes on the denuded basement membrane is seen Neutrophils adhere to the injured capillary endothelium and extravasate into alveoli, which is filled with protein rich edema fluid. In the alveoli, alveolar macrophages secrete cytokines, which further stimulate chemotaxis and activate neutrophils. Neutrophils release oxidants, proteases, leukotrienes, and other proinflammatory mediators, such as platelet activating factor. The influx of protein rich edematous fluid into the alveolus leads to the inactivation of surfactant.

Reproduced with permission. Ware, L.B. \& Matthay, M.A. The acute respiratory distress syndrome. N Engl J Med 342, 1334-1349 (2000). ${ }^{4}$ 


\subsubsection{Pulmonary Cytokines and Inflammation in ALI}

A complex network of cytokines and other pro-inflammatory compounds initiate and amplify the inflammatory response in ALI. Different cell types, such as endothelial, epithelial and leukocytes produce cytokines and chemokines. Excessive or unregulated release of cytokines and chemokines might result in unwanted systemic and/or local effects that may cause ALI. The inflammatory process is regulated by the early response cytokines (e.g. tumor necrosis factor- $\alpha$ (TNF- $\alpha$ ), IL-1 $\beta$ and IL-6), which are suggested as key mediators in the pathogenesis of ALI. ${ }^{10}$ These early response cytokines induce an intricate cascade of events, which include the production and release of chemokines (e.g. IL-8, monocyte chemoattractant protein-1 (MCP-1), macrophage inhibitory protein-1 (MIP-1) and cytokine induced neutrophil chemoattractant-1 (CINC-1)) from endothelial cells, epithelial cells or leukocytes. ${ }^{4,11-13}$ In addition, cytokines and chemokines cause sequestration of leukocytes by inducing adhesion molecules (e.g. integrins, selectins and members of immunoglobulin superfamily) expression on epithelial and endothelial cells, and further activate the leukocytes, which trigger an extensive inflammation process. ${ }^{14-17}$

\subsubsection{Pulmonary Edema}

In the normal lung, fluid moves continuously outward from vessels to the interstitial space according to the permeability of the capillary membrane as well as the net difference between hydrostatic and protein osmotic pressures. Non-cardiogenic pulmonary edema occurs when the permeability of the alveolar epithelial/endothelial membrane increases because of direct or indirect lung injury resulting in a marked increase in the amount of fluid and protein leaving the vascular space. As illustrated in Figure 1.2, nature of the inflammatory stimulus/injury determines the mechanistic pathway for an increase in permeability across alveolar epithelium or endothelium, thus contributing to pulmonary edema.

\subsubsection{Alveolar Epithelial Integrin Receptors: Pulmonary Edema}

Pittet et al. showed that lipopolysaccharide (LPS) and IL-1 $\beta$ cause an increase in lung vascular permeability. Both the stimuli exert this effect through activation of transforming growth factor-beta (TGF- $\beta$ ) via $\alpha v \beta 6$ integrin dependent mechanism in alveolar epithelium. Under normal conditions, TGF- $\beta$ is latent and is non-covalently associated with latency associated peptide (LAP) and latent TGF- $\beta$-binding protein-1 (LTBP-1). Activation of TGF- $\beta$ by integrin receptors is critical in the propagation of lung edema in ALI. ${ }^{18-20}$ The $\alpha v \beta 6$ integrin binds to RGD domain of the LAP of TGF- $\beta$ complex and activates latent TGF- $\beta .{ }^{19}$ Although, the exact mechanism of $\alpha v \beta 6$ integrin mediated TGF- $\beta$ activation is still not clear, it is likely that the activation process involves multiple coordinated mechanisms.

Pittet et al. demonstrated that $\alpha \mathrm{v} \beta 6$ integrin mediated TGF- $\beta$ activation is dependent on cytoskeletal integrity. Inhibition of actin assembly by cytochalasin D and $\beta 6$ subunit 


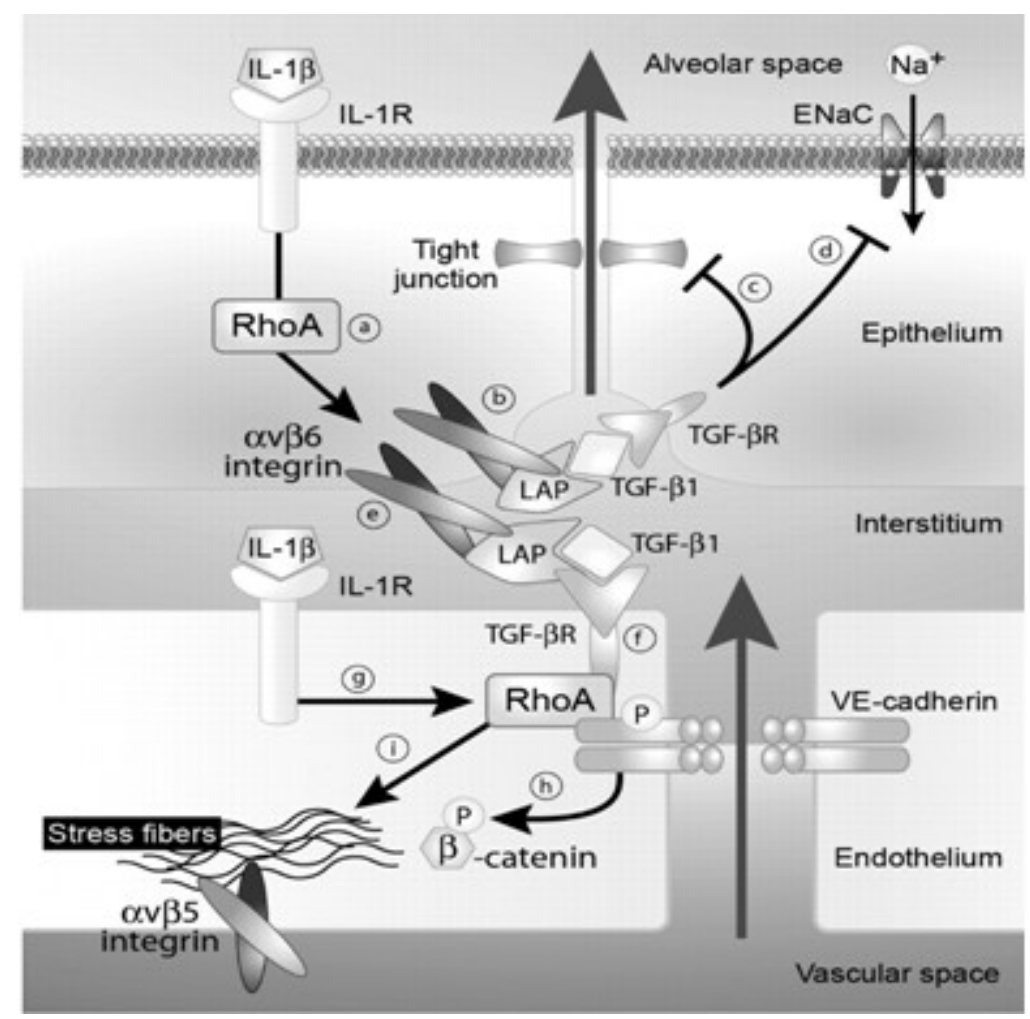

Figure 1.2 Schematic illustration of the effect of interleukin-1 $\beta$ on the alveolar capillary barrier.

The model demonstrates IL- $1 \beta$ signaling pathway that leads to an increase in lung epithelial and endothelial permeability. IL-1 $\beta$ causes an increase in RhoA activity leading to TGF- $\beta$ activation via $\alpha \mathrm{v} \beta 6$ integrins. TGF- $\beta$ activation induces an increase in permeability and an inhibition of the $\mathrm{Na}^{+}$driven fluid transport in alveolar epithelial cells. In endothelial cells, IL-1 $\beta$ activates RhoA and increases lung endothelial permeability via the phosphorylation and endocytosis of $\beta$-catenin and $\alpha v \beta 5$ integrin dependent stress fiber formation in lung endothelial cells.

Reproduced with permission. Ganter, M.T. et al. Interleukin-1 \{beta\} causes acute lung injury via $\{$ alpha $\}$ v $\{$ beta $\} 5$ and $\{$ alpha $\}$ v $\{$ beta $\} 6$ integrin-dependent mechanisms. Circ Res 102, 804-812 (2008). ${ }^{21}$ 
cytoplasmic truncation mutants prevent integrin interaction with actin cytoskeleton, each abolishes TGF- $\beta$ activation by $\alpha v \beta 6 .{ }^{19}$ Jenkins et al. demonstrated that upon external stimulus, PAR1 receptor signals to the $\alpha v \beta 6$ integrins via RhoA and Rho kinase. ${ }^{20}$ Because of the well-known effects of RhoA in re-organizing actin, these findings are consistent with the observation that $\alpha \mathrm{v} \beta 6$ mediated TGF- $\beta$ activity is completely inhibited by an inhibitor of actin polymerization, cytochalasin D. In fact, blockade of any component in the sequence of events in actin polymerization leads to inhibition of TGF- $\beta$ activity. A complete blockade of TGF- $\beta$ activity was observed using $\alpha v \beta 6$ antibodies. Recently, Ganter et al. demonstrated a complete inhibition of pulmonary edema using anti- $\alpha v \beta 6$ integrin and anti-TGF- $\beta$ antibodies in IL- $1 \beta$ instilled ALI model. ${ }^{21}$

\subsubsection{Alveolar Endothelial Integrin Receptors: Pulmonary Edema}

Physical passage of solutes through the endothelial barrier is thought to occur via paracellular pathways or through receptor activated transcytosis. ${ }^{22}$ Direct modification of actin cytoskeleton in endothelial cells is important for increasing paracellular permeability. One frequently cited model describes paracellular gap formation as a consequence of imbalanced competition between cytoskeletal, adhesive cell-cell and cell-matrix forces. ${ }^{23,24}$ Actomyosin mediated generation of tension leads to alteration of cell shape and formation of paracellular gaps. Stress fibers have been shown to form in endothelial cells, stimulated by several vasoactive mediators. ${ }^{21,25,26}$ In TGF- $\beta$ stimulated model, TGF- $\beta$ activates RhoA signaling cascade in endothelial cells. The TGF- $\beta$ induced activation of the RhoA/Rho-kinase signaling cascade in endothelial cells increases Factin content, which contributes to an increase in stress fiber formation, interendothelial gap formation and subsequent decrease in endothelial monolayer resistance. ${ }^{27}$ Ganter et al. demonstrated that IL-1 $\beta$ activates RhoA, and increases lung endothelial permeability via $\alpha v \beta 5$ integrin dependent stress fiber formation in lung endothelial cells. Furthermore, stress fiber formation induced by the agonists was attenuated by blockade of $\alpha \mathrm{v} \beta 5$ integrin, suggesting a mechanism for $\alpha v \beta 5$ integrins to regulate paracellular endothelial permeability in the lung. A complete inhibition of transendothelial permeability was observed using anti- $\alpha \mathrm{v} \beta 5$ integrin antibodies. ${ }^{21}$

In summary, $\alpha v \beta 5 / \alpha v \beta 6$ integrins play a critical role in mediating the development of pulmonary edema in LPS or IL- $1 \beta$ or TGF- $\beta$ stimulated ALI models. Taken together, transient blockade of both integrins by antibodies or small RGD peptides may provide new causal therapies for pulmonary edema.

\subsubsection{Leukocyte Migration in Pulmonary Vasculature}

Circulating leukocytes infiltrate from blood circulation into tissues under both normal and pathologic circumstances. As illustrated in Figure 1.3, leukocyte migration from the vasculature occurs by a multi-step process, dictated by the sequential activation of adhesion molecules and their ligands on both leukocytes and ECs. ${ }^{28,29}$ Leukocytes 


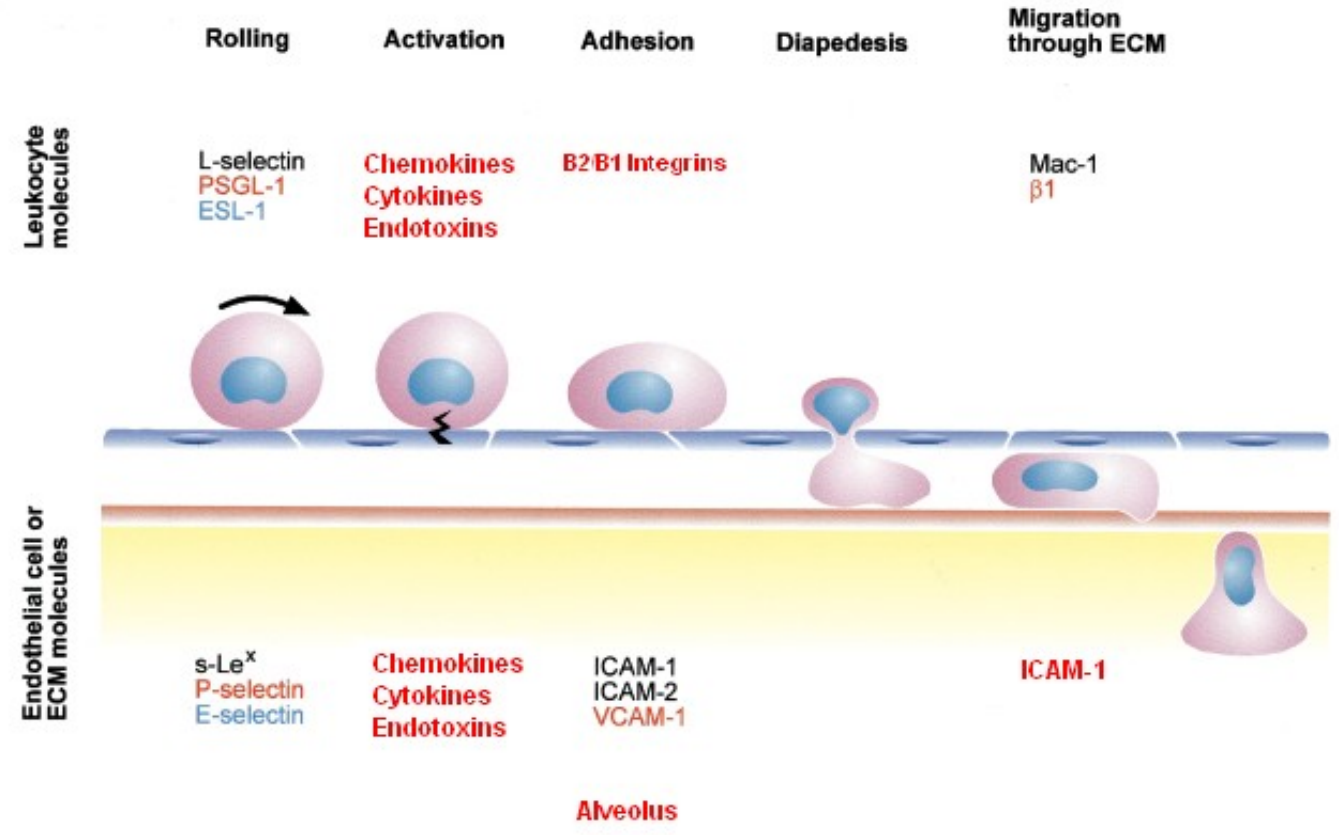

Figure 1.3 Schematic diagram of neutrophil and endothelial cell interactions in the pulmonary vasculature.

E-selectin upregulation induces rolling of neutrophils, which adhere to endothelial cells activated by ICAM-1. Followed by transendothelial migration, neutrophils infiltrate into alveoli. Schematic diagram also shows various receptors and ligands involved in the neutrophil migration process. 
infiltrate by these sequence dependent mechanisms but differ in their responses to chemotactic (e.g. MCP-1) and cytokine signals (e.g. TNF- $\alpha$ or IL-1 $\beta$ ), particularly in their qualitative and quantitative expression of adhesion molecules (e.g. selectins, integrins and members of immunoglobulin superfamily). ${ }^{30-32}$ Leukocyte trafficking in pulmonary circulation differs from systemic vasculature with respect to: I) the marginated pool of leukocytes, II) the site of transendothelial migration and III) requirements of adhesion molecules for leukocyte migration. Leukocyte migration begins with the "capture" by the vessel wall of leukocytes from flowing blood and is followed by their "rolling" to a small extent along the vessel wall. About $97 \%$ of pulmonary vascular leukocytes $(7-8 \mu \mathrm{m})$ are found in the capillary network where vessels are too small to allow rolling $(5-6 \mu \mathrm{m}) .{ }^{33}$ Despite the lack of rolling in pulmonary capillaries, selectins may be involved in the leukocyte-endothelial cell interactions. ${ }^{34-36}$ Only after appropriate stimuli from alveolar capillary ECs, leukocytes become firmly adhered to ECs and are positioned for migration from blood vessels into lung parenchyma.

The initiating signal for the next step of migration is firm adhesion, which is postulated to be either a receptor mediated event in response to pro-inflammatory cytokines/chemokines or an event propagated from signals from activated selectins. ${ }^{36-38}$ Cytokines/chemokines on inflamed endothelium interact with their receptors on leukocytes. These interactions result in the activation of leukocyte integrins. Activated integrins interact with members of the immunoglobulin superfamily (e.g. ICAM-1) displayed on the inflamed endothelium, and these interactions cause the rolling cells to arrest on endothelium. Transmigration of arrested leukocytes across the endothelium is not as well understood. However, it is generally accepted that leukocytes migrate across the endothelium by penetrating junctions that lie within the intercellular cleft. Leukocytes move across blood vessel walls by passing between ECs (diapedesis). ${ }^{39}$ After transmigration, leukocytes move into the interstitium along a gradient of chemoattractant toward the source of the chemoattractant. In lungs, leukocytes then migrate across the epithelium to reach the apical side. Molecular requirements of transepithelial migration are not well understood but most likely involve a multi-step process that requires sequential interactions of leukocyte adhesion molecules with their ligands on the epithelial cells. ${ }^{40,41}$

Leukocyte migration depends on: I) nature of inflammatory stimuli and II) route of exposure of stimuli to the alveolar capillary bed. Doerschuk et al. demonstrated that neutrophil migration occurs through at least two distinct pathways. One pathway requires $\beta 2$-integrins, whereas the other does not, and the distinction depends on the stimulus. ${ }^{42}$ Gram negative bacteria (e.g. E.coli) or LPS elicit neutrophil migration via pathways predominately mediated by $\beta 2$ integrins. ${ }^{42}$ Alveolar endothelial ICAM-1, which serves as a ligand for neutrophil $\beta 2$ integrins is upregulated in response to LPS stimulus. In contrast, gram-positive bacteria (e.g. S.pneumoniae) elicit pulmonary neutrophil migration independent of $\beta 2$ integrins. ${ }^{43}$ In a situation where neutrophil migration is $\beta 2$ integrin independent, antibody inhibition of $\beta 1$ integrin or specific $\alpha$-subunits $(\alpha 2, \alpha 4$, $\alpha 5$ and $\alpha 6$ ) blocks neutrophil migration into alveoli. ${ }^{44}$ Moreover, gram-negative stimuli appear to elicit greater TNF- $\alpha$ production than do gram-positive stimuli. Taken together, integrin antibody studies and disparate cytokine production elicited by various stimuli 
suggest that the adhesion molecule requirements for migration might depend on the type and quantity of cytokines and chemokines produced in response to inflammatory stimulus.

Shang and Issekutz identified a role for $\beta 2$ integrins, as well as $\alpha 4, \alpha 5$ and $\alpha 6 \beta 1$ integrins. ${ }^{45}$ With a cocktail of blocking antibodies to these integrins, neutrophil migration was inhibited by $60 \% .{ }^{46}$ Recently, a few studies indicated exceptions to the $\beta 2$ integrin dependent neutrophil migration in LPS stimulated models. These studies reported the significance of $\alpha 4$ and/ or $\alpha 5$ integrins along with $\beta 2$ integrins. ${ }^{47,48}$ Intratracheal (i.t.) instillation of LPS (E.coli) results in $\beta 2$ integrin dependent migration, while intravenous administration results in sequestration that is $\beta 2$-integrin independent. ${ }^{42,49}$ Airway instillation of stimuli via trachea would presumably induce inflammation along airway walls containing vessels of the bronchial circulation as well as pulmonary capillary beds. Conversely, models that use intralobular instillation may deliver inflammatory stimuli anatomically closer to alveolar regions, and may therefore induce relatively more neutrophil migration from the pulmonary vasculature.

Clinical and experimental studies have provided circumstantial evidence of the occurrence of neutrophil mediated injury in ALI. Broncho-alveolar lavage fluid (BALF) in normal subjects yields normally only a few neutrophils. ${ }^{50}$ However, a subpopulation of circulating neutrophils is always present within the pulmonary microvasculature. Evidence shows that percentage of BALF neutrophils is markedly increased in ALI affected patients. ${ }^{51}$ The migrated neutrophils release myeloperoxidase (MPO) and reactive oxygen species (ROS) in alveoli, which can be potentially harmful to alveolar epithelium. ${ }^{52}$ When produced in excessive amounts or when anti-oxidant defense is insufficient, free radicals can damage DNA, lipids, proteins and carbohydrates. ${ }^{53}$ Alongside neutrophils, monocytes play a critical role in the propagation of inflammatory response. ${ }^{54,55}$ From blood circulation, they migrate into lungs and differentiate into alveolar macrophages, which amplify the inflammatory response through the production and secretion of chemokines and other pro-inflammatory mediators.

\subsubsection{Relevance of ALI Models in Therapeutic Intervention}

Different animal models of ALI are in use to investigate pathological mechanisms of lung injury. ${ }^{56}$ However, none of the ALI models adequately reproduces the full characteristics of human ALI, and therefore, the choice of a particular model is made after considering the specific features of each model. Most ALI models target the primary tissues, alveolar epithelium or endothelium or both. Modeling strategies are an attempt to target specific tissue. From a practical standpoint, there are three general types of model systems: I) models in which the lung is injured directly by a noxious stimulus. These include intratracheal or intranasal administration of bacteria or bacterial products, such as LPS or pro-inflammatory cytokines, IL-1 $\beta$; administration of an acid, such as $\mathrm{HCl}$ to reproduce aspiration; ${ }^{57,58}$ administration of high inspired fractions of oxygen $;{ }^{59}$ depletion of surfactant by serial lavage with $0.9 \% \mathrm{NaCl}^{60}$ or exposure to mechanical stretch using mechanical ventilation with high tidal volumes, ${ }^{61}$ II) models in which the lung is injured 
indirectly. This category includes models based on reproducing sepsis, such as cecal ligation and puncture (CLP) ${ }^{62}$ systemic administration of LPS or bacterial infusion ${ }^{63}$ and III) combination models. Most commonly used model includes saline lavage followed by mechanical ventilation, or CLP followed by hemorrhage.

Exposure to moderate doses of LPS is widely used as a prototypic ALI model,${ }^{64}$ while administration of high doses of LPS is considered as a relevant model of ARDS ${ }^{65}$ Early phase (4-6 h) of LPS instillation (i.t.) is characterized by an increase in BALF leukocytes, albumin and pro-inflammatory cytokines/chemokines, and late phase (24-48 h) is characterized by normalization of BALF cytokines and increase in BALF leukocyte counts. ${ }^{66}$ Systemic administration of LPS in animals induces a septic shock resulting in injury to various organs, including the liver ${ }^{67}$ and the lung. ${ }^{68}$ Following the systemic administration of LPS, the capillary endothelium is the initial site of injury, which results in apoptosis of endothelial cells. ${ }^{69}$ Neutrophil migration occurs before epithelial permeability changes or alveolar barrier disruption..$^{70}$ Other hallmark features include elevated concentrations of cytokines/chemokines in blood circulation and coagulation abnormalities. ${ }^{71}$ Altogether, these animal models clearly demonstrate that direct (pulmonary) or indirect (systemic) challenges by endotoxins may cause lung tissue damage that are severe enough to qualify as ALI/ARDS. In addition, animal models provide a critical method for testing potential new therapies in a proof-of-principle fashion.

\subsubsection{Pharmacological Interventions of ALI in Preclinical and Clinical Trials}

The pathophysiology of ALI is increasingly well understood; however, very little success was achieved with regard to new treatment modalities. The spate of trial failures may relate to participant heterogeneity or to intervention strategy. Important considerations for trial failures are the timing of intervention (pre-ALI, the early exudative phase, the intermediate fibro-proliferative phase or later), mechanism of drug delivery (intravenous, enteral, inhaled or intrapulmonary instillation). As new information regarding the pathogenesis of ALI evolves, more specific therapies are being studied, including separate considerations for direct or indirect forms of ALI. Table 1.1 is a summary of the therapies under preclinical and various phases of clinical development.

\subsubsection{Anti-inflammatory Strategies: Corticosteroids}

Transcription factor, NF-kB is associated with the regulation of a battery of genes that encode for cytokines, chemokines and adhesion molecules. ${ }^{72,73} \mathrm{NF}-\mathrm{kB}$ is a heterodimeric protein composed of the DNA binding proteins, p65 and p50 constitutively present in the cytoplasm in an inactive form stabilized by the binding to the inhibitory protein, IKB $\alpha .^{74}$ Cellular activation by adverse stimuli leads to phosphorylation and degradation of I $\kappa \mathrm{B} \alpha .{ }^{75}$ The liberated NF- $\kappa \mathrm{B}$ then translocates into the nucleus and binds to promoter regions of target genes to initiate the synthesis of the pro-inflammatory cytokines, chemokines and adhesion molecules. 
Table 1.1 Pharmacological agents in preclinical and clinical phases of drug development.

\begin{tabular}{ll}
\hline Stage of study & Pharmacological intervention \\
\hline Preclinical & Cyclooxygenase inhibitors: Eltenac \\
& Keratinocyte growth factor (KGF) \\
& Neutrophil elastase inhibitors: EPI-HNE-4 \\
Phase I & Anti-oxidant: OxSODrol \\
& Cytoprotective therapies: CPC-111 \\
& Soluble complement receptors: TP-20 \\
Phase II & Anti-coagulants: NAPc2 \\
& Anti-inflammatory: PAF-AH/Pafase \\
& Inhaled nitric oxide \\
& B2-agonists \\
& Soluble complement receptors: TP-10 \\
& Liquid ventilation: LiquiVent \\
& Albumin and furosemide \\
& Nutrition \\
& Surfactants: Venticute ${ }^{\mathrm{TM}}$, HL-10, lucinactant \\
& Prostaglandins: Prostacyclin/PGI2, PGE1/TLC C-53 \\
& Growth Factors: GM-CSF/leukine \\
& Anti-coagulants: rhAPC/drotrecogin alfa, TFPI \\
& Corticosteroids \\
\hline
\end{tabular}

G-CSF: Granulocyte colony-stimulating factor; GM-CSF: Granulocyte macrophage colony-stimulating factor; hLS: Human lung surfactant; HNE: Human neutrophil elastase; KGF: Keratinocyte growth factor; NAC: N-acetylcysteine; NAP: Anticoagulation protein; rhAPC: Recombinant human activated protein C; PAF-AH: Platelet activating factor acetylhydroxylase; TFPI: Tissue factor pathway inhibitor. 
Glucocorticoids mediate NF- $\kappa \mathrm{B}$ transcription interference by one of the following mechanisms $\left.:^{75} \mathrm{I}\right)$ physically interacting with the p65 subunit and formation of an inactive (GR-NF- $\kappa \mathrm{B}$ ) complex, ${ }^{76} \mathrm{II}$ ) inducing the transcription of the inhibitory protein, $\mathrm{I} \kappa \mathrm{B} \alpha{ }^{76-78}$ III) impairing TNF- $\alpha$ induced degradation of I $\mathrm{B} \alpha^{79}$ and (IV) competing for limited amounts of GR $\alpha$ co-activators, such as steroid receptor co-activator- $1 .{ }^{80}$ Through one of the above mechanisms, GCs suppress the production of cytokines by target cells (e.g., endothelial, epithelial and leukocytes) in response to inflammatory stimuli. Furthermore, they downregulate the expression of chemokines and adhesion molecules on these cells. $^{72,81-83}$ Studies in vitro demonstrate a direct effect of GCs on cultured ECs and isolated neutrophils to inhibit expression of adhesion molecules. ${ }^{84-86}$ Several case series reports ${ }^{87,88}$ suggested that glucocorticoids could lower mortality in some patients with severe ALI when administered several days after ALI onset. In a small, randomized, placebo-controlled trial important clinical outcomes were better in patients randomized to receive methylprednisolone in the late phase of ALI. ${ }^{89}$

\subsubsection{Prostaglandin Agonists/Inhibitors}

Prostaglandin E1 is a vasodilator that blocks platelet aggregation and decreases neutrophil activation. This agent showed promise in experimental and preliminary clinical studies of lung injury. ${ }^{90}$ However, a multi-center study ${ }^{91}$ reported no evidence of reduced mortality in those treated with prostaglandin E1. Liposomal delivery of prostaglandin E1 was also not beneficial in a phase II study. ${ }^{92}$ Ketoconazole, a potent inhibitor of thromboxane and leukotriene synthesis was reported to prevent the development of ALI in high risk surgical patients. However, an NIH-sponsored multicenter phase III trial ${ }^{93}$ showed no decrease in mortality for ketoconazole treatment $(35 \%)$ vs. the placebo group (34\%).

\subsubsection{Phosphodiesterase Inhibitors}

Pentoxifylline is a phosphodiesterase inhibitor that inhibits neutrophil chemotaxis and activation. ${ }^{94}$ In animal studies, lisofylline inhibited release of TNF- $\alpha$, IL-1 $\beta$ and IL- 6 , and attenuated shock induced lung injury in mice. ${ }^{95}$ However, phase III trial ${ }^{96}$ by the NIHARDS Network in ALI patients showed no beneficial effects of lisofylline.

\subsubsection{Anti-oxidants}

Evidence suggests that ROS plays a major role in mediating injury to the endothelial barrier of the lung in ALI. ${ }^{97}$ In normal individuals, oxidant productions are normally balanced by a number of anti-oxidants, including water soluble molecules, such as glutathione and lipid soluble molecules, such as Vitamin E ( $\alpha$-tocopherol). ${ }^{98,99} \mathrm{~N}$ acetylcysteine (NAC), a synthetic anti-oxidant mediates its effect as a precursor for gluthatione. ${ }^{98}$ NAC was shown to protect lung tissue against oxidative damage in preclinical models. ${ }^{100}$ However, clinical studies failed to improve survival in ALI 
patients. ${ }^{101}$ Vitamin E, considered as the principal anti-oxidant improved immune responsiveness, and reduced the incidence and severity of infectious diseases. ${ }^{102}$

\subsubsection{Anti-IL-8 Therapy}

One approach is to reduce the number of neutrophils that migrate into the extravascular space of the lung by interfering with neutrophil adhesion to the lung endothelium or by reducing the release of chemotactic factors in the extravascular space. Monoclonal antibodies that neutralize IL-8 reduced acid-induced lung injury in rabbits. ${ }^{103}$ Clinical trials of anti-IL-8 therapy for prevention in high risk patients or in early ALI may soon be warranted.

\subsubsection{Surfactants}

Surfactant is a lipoprotein complex produced by alveolar type II cells. Alveolar surfactant secretion reduces surface tension, and prevents alveoli from collapsing. ${ }^{104}$ Whether reduced in amount or dysfunctional in condition, surfactant abnormalities may lead to problems, such as ventilation-perfusion mismatch, hypoxemia and reduced lung compliance. ${ }^{105}$ Surfactant replacement therapy in animal models of ALI improved oxygenation, lung mechanics and survival. ${ }^{104,106}$

\subsubsection{Miscellaneous Therapies}

Many other therapeutic interventions are under preclinical and clinical investigations. Partial liquid ventilation uses perfluorocarbons to maintain open lung units and improve oxygenation. ${ }^{107}$ The use of activated protein $\mathrm{C}^{108}$ and GM-CSF ${ }^{109}$ are all moving forward in large scale clinical trials. $\beta 2$ agonists ${ }^{110}$ and fluid balance manipulation ${ }^{111}$ appear promising despite varied conclusions from previous trials. Various ligand based pharmaceuticals (e.g. inhibitors of the upregulated receptors on alveolar epithelium or endothelium or leukocytes) are in different stages of drug development.

Citing the multiple pathways involved in ALI pathogenesis, an 'all-out' therapy that contributes to the resolution of edema, and inhibition of leukocyte migration and proinflammatory mediators' production is most desirable rather than just limiting one or the other.

\subsubsection{Significance of Drug Delivery in ALI Pathogenesis}

Numerous clinical trials involving pharmacological therapies aimed at different pathological targets turned unsuccessful. One of the attributed drawbacks is the underappreciation of drug delivery systems in the management of ALI. Very few investigations were directed at drug delivery perspective in the treatment of ALI. However, the results 
are promising both in the alleviation of the syndrome and improving the survival of patients. As described previously, the alveolar epithelium and endothelium play an important role in the vicious circle of production of pro-inflammatory mediators and leukocyte recruitment. An attractive therapeutic approach is to selectively target antiinflammatory drugs to activated epithelium/endothelium, thereby increasing the effectiveness of the targeted drug and simultaneously diminishing systemic side effects. To selectively deliver drugs into activated alveolar epithelial/endothelial cells, integrins and ICAM-1 receptors are suitable targets because of spatial and temporal expression in response to inflammatory stimulus. Furthermore, both integrin and ICAM-1 receptors are internalizing molecules, which results in the intracellular degradation of the drugtargeting systems and drug release, thereby leading to local anti-inflammatory activity. Thus, targeting to integrin/ICAM-1 receptors facilitates multi-purpose: I) specific delivery of drugs for inhibition of pro-inflammatory mediators' production and II) blockade of receptors for inhibition of pulmonary edema/leukocyte migration.

Optimal targeted drug delivery systems incorporate the ability to preferentially recognize and localize to sites of injury, while simultaneously avoiding uptake into normal tissue beds. ${ }^{112}$ Therefore, active targeted drug delivery systems are investigated to improve therapeutic efficiency and reduce side effects associated with systemic administration. Liposomes are studied extensively as potential vehicles for selective drug targeting, achieved by modulation of specific and non-specific cellular and molecular interactions. Liposomal drug delivery systems have received considerable attention due to their specific advantages: I) encapsulation of small and large molecules with a wide range of hydrophobicity levels and $\mathrm{pKa}$ values, II) prolong and target therapeutic agents by modification of liposome surface and III) minimize clinical drug dose and reduce toxicity effects. The surface chemistry of liposomes can be controlled by the selection of particular phospholipid moieties to incorporate several different molecules in well defined molar ratios. As a result, liposomes can be engineered to provide great diversity of structures with exceptional design control. Liposomes also have a relatively high encapsulation volume available for carrying therapeutic agents compared with other drug carrying nano sized vehicles. ${ }^{113}$ Therefore, liposomes are considered as potential targeted drug delivery systems.

In the current investigation, we will apply the liposome drug delivery perspective to the pathological mechanisms, and improve therapeutic benefits in the treatment of ALI. It is perhaps this requirement that is most tenuous, but the one with the great potential.

\subsection{Overview of Liposome Literature}

Most of the medical applications of liposomes are in cancer and inflammation areas. Ligand targeted liposomes perform best against targets in the vasculature or readily accessible from the vasculature, e.g. as an adjuvant therapy in the treatment of residual and micro-metastatic disease following primary therapy of solid tumors by surgery or radiation. ${ }^{114}$ Over the next decade, the number of liposome based pharmaceutical products will continue to expand, justifying the investment in research and development 
over the previous years. Table 1.2 illustrates the liposome products in preclinical and clinical phases of development.

\subsubsection{Basic Liposome Composition}

Liposomes are spherical self-closed vesicular structures. The size of a liposome ranges from $20 \mathrm{~nm}$ to $1-5 \mu \mathrm{m}$, and they may be composed of one or several concentric membranes, each with a thickness of about $4 \mathrm{~nm}$. Liposomes possess unique properties owing to the amphipathic character of the lipids, which make them suitable for drug delivery. Liposomes, prepared using a lipid or combination of lipids carry water soluble drugs in their aqueous compartments and lipid soluble drugs in their lipid bilayers. The amphipathic phospholipid molecules, with distinct hydrophobic and hydrophilic domains, spontaneously form bilayers in aqueous media as a consequence of the interaction between the polar head group of the phospholipid and water. The charge, phase transition, hydrogen-bonding capacity, membrane rigidifying potential and functional groups of phospholipids all affect the retention of the encapsulated drug.

The inclusion of cholesterol (CHOL) stabilizes liposomal phospholipid membranes against disruption by plasma proteins ${ }^{115,116}$ and results in decreased binding of plasma opsonins responsible for rapid clearance of liposomes from circulation. ${ }^{117} \mathrm{Cullis}$ and coworkers $^{118}$ showed that CHOL-free liposomes bound 3-4 times more protein than CHOL containing liposomes. This increase in CHOL caused a substantial increase in clearance from the blood of mice. CHOL-free liposomes were shown to aggregate rapidly in the absence of steric stabilization, and further leads to increased clearance. ${ }^{119}$ The fluidity of the membrane also affects the rate of clearance, with non-pegylated liposomes composed of unsaturated phospholipids and CHOL display rapid clearance rates than those containing saturated phospholipids. ${ }^{120-122}$ In the absence of CHOL, neutral phosphatidylcholine liposomes composed of saturated lipids were cleared more rapidly. ${ }^{118}$ However, high CHOL concentrations (30 mole\%) resulted in the formation of a highly ordered crystalline state, which decreased protein binding and dramatically increased circulation life times. ${ }^{118}$ The rate of liposome clearance varied positively with the phase transition temperature of the phosphatidylcholine. ${ }^{118}$ This is likely due to increased protein binding to exposed hydrophobic domains originating from packing defects in gel phase membranes. Substitution of sphingomyelin for the high phase transition distearoyl phosphatidylcholine (DSPC) increased circulation life times in CHOL containing liposomes. ${ }^{123}$ This is likely due to the highly cohesive membranes ${ }^{124}$ formed as a result of intermolecular hydrogen bonds between sphingomyelin and neighboring $\mathrm{CHOL}^{122,125,126}$ or even other sphingomyelin ${ }^{127}$ molecules. Liposomes composed of hydrogenated sphingomyelin and CHOL were shown to improve circulation life times of vincristine encapsulated liposomes. ${ }^{128}$

Charged lipids affect liposome clearance, although the relationship is often very complex. ${ }^{122}$ In general, low concentration of charged lipids provide sufficient electrostatic repulsion to prevent liposome aggregation upon addition of hydrophobic drugs to the membrane. ${ }^{129}$ However, the inclusion of high concentration of either anionic 
Table 1.2 Liposome products in clinical and preclinical studies.

\begin{tabular}{|c|c|c|}
\hline Product & Application & Company; trial phase \\
\hline \multicolumn{3}{|l|}{ Clinical studies } \\
\hline \multicolumn{3}{|l|}{ Drug delivery } \\
\hline Caelyx & Bladder cancer & Schering Plough; EU \\
\hline Doxil & Ovarian cancer & Ortho Biotech \\
\hline Doxil+VAD & Myeloma & Millennium \\
\hline Doxil+VAD & Multiple myeloma & Millennium; III; J\&J; III \\
\hline Doxil+Yondelis & Ovarian cancer & J\&J; III \\
\hline Myocet & Breast cancer & Sopherion Therapeutics; III \\
\hline Liposomal cisplatin & Recurrent ovarian cancer & Sequus; II \\
\hline Aroplatin ${ }^{\mathrm{TM}}$ & B-cell lymphoma & Antigenics; I \\
\hline Annamycin & Leukemia & Aronex Pharm; II \\
\hline Paclitaxel & Head and neck cancer & Neopharm; II and III \\
\hline Vincristine & Lung cancer & Inex Pharm; II \\
\hline Topoisomerase inhibitor & Lung and ovarian cancer & OSI Pharm; II \\
\hline Mitoxanthrone & Other cancers & Neopharm; II \\
\hline Nystatin & Leukemia & Pfizer; III \\
\hline \multicolumn{3}{|l|}{ DNA delivery } \\
\hline $\begin{array}{l}\text { Interleukin-2 plasmid DNA } \\
\text { Antigen delivery }\end{array}$ & Kidney and prostate cancer & Vical; II \\
\hline MUC-1 peptide; BLP25 & Lung cancer & Merck; III \\
\hline \multicolumn{3}{|l|}{ Preclinical studies } \\
\hline \multicolumn{3}{|l|}{ Drug delivery } \\
\hline Adriamycin & Sarcoma & \\
\hline Doxorubicin & Neuroblastoma & \\
\hline Vincristine & B-cell lymphoma & \\
\hline Prednisolon & Autoimmunity & \\
\hline Clodronate & Autoimmunity & \\
\hline Glucocorticoids & Arthritis & \\
\hline Glucocorticoids & ALI & \\
\hline Budesonide & Asthma & \\
\hline Tacrolimus & Skin disorders & \\
\hline Interleukin-2 & Immunotherapy & \\
\hline \multicolumn{3}{|l|}{ DNA delivery } \\
\hline Bcl-2 antisense ODNs & Cancer & \\
\hline Interferon-b DNA & Glioma & \\
\hline
\end{tabular}

VAD denotes vincristine, adriamycin and doxorubicin (DXR) combination 
or cationic lipids into liposomal membrane increases clearance of liposomes from the circulation. High concentration of anionic lipids increases accumulation in liver and spleen. ${ }^{122,130}$ In addition to liver and spleen, cationic lipids enhance uptake by vascular endothelial cells of lung, ${ }^{131}$ tumor vasculature ${ }^{132}$ and blood brain barrier. ${ }^{133}$ In anionic liposomes, net negative charge of liposomes is dependent on the specific anionic lipid utilized in the formulation, with low concentrations of anionic gangliosides, phosphatidylglycerol (PG), phosphatidylinositol (PI) and PEGylated phosphatidylethanolamine (PEG-PE) result in substantial reduction in clearance. ${ }^{120,122,130,134}$ The presence of anionic lipids, such as phosphatidic acid (PA), phosphatidylserine (PS) and cardiolipin (CL) caused an increase in the rate of clearance from the blood. ${ }^{122,130,135}$ This discrepancy is in part due to the differences in protein/opsonins binding to membranes containing different anionic lipids. Liposomes comprised of the anionic lipids exhibited high plasma protein binding, while liposomes composed of PG, PI and PEG-PE show less protein binding. ${ }^{130}$ The presence of PS on liposomal surface resulted in receptor-mediated clearance. ${ }^{136}$ Therefore, caution should be exercised in the selection of charged lipid components in liposomal therapeutics.

The phase transition temperature (Tm) of the phospholipid component influences drug retention. The phospholipid undergoes a shift from a well ordered gel phase to a fluid and disordered liquid crystalline state at its phase transition temperature. In general, liposome membranes are more permeable above the Tm of the particular phospholipid. For unsaturated phospholipids, physiological temperature is above the phase transition, and therefore, the drug retention is compromised. On the other hand, saturated phospholipids, such as DSPC or hydrogenated soy phosphatidylcholine (HSPC) have a phase transition temperature $\left(50-58^{\circ} \mathrm{C}\right)$ above the physiological temperature, and therefore, the liposome formulations are stable. The clearance rates of doxorubicin loaded pegylated liposomes were correlated with the Tm of the phosphatidylcholine (EggPC $>$ DPPC $>$ HSPC), reflecting the increased membrane permeability, and thus leakage of doxorubicin for the EggPC and dipalmitoyl phosphatidylcholine (DPPC) formulations. ${ }^{137}$

Sphingomyelin stabilizes membranes in the presence of CHOL. It imparts compact packing of membrane lipids ${ }^{138}$ with a decrease in permeability to water and other solutes. ${ }^{139}$ The high cohesiveness and reduced permeability of the membrane could be due in part to the strong hydrogen bonds between the amide nitrogen of sphingomyelin and the hydroxyl group of CHOL. ${ }^{126}$ In addition, due to the amide linkage in its backbone, sphingomyelin is also less sensitive to $\mathrm{pH}$-dependent hydrolysis. ${ }^{140}$ Substitution of dihydrosphingomyelin for sphingomyelin was shown to improve the stability of vincristine encapsulation. Thus, the ability of sphingomyelin and dihydrosphingomyelin to reduce the membrane permeability of certain drugs can play an important role in regulating the rate of drug release for certain small molecule drugs.

\subsubsection{Physico-chemical Properties of Drugs: Liposome Encapsulation}

The physico-chemical properties of the drug to be incorporated into liposomes play an important role in determining its rate of release. Encapsulated drugs are classified as: (I) 
water insoluble hydrophobic drugs, (II) amphipathic weak bases, (III) amphipathic weak acids, (IV) highly water and membrane impermeable small molecular weight drugs and (V) polyions, such as nucleic acids. The most commonly studied drugs are the amphipathic weak bases (e.g. camptothecins, anthracyclines). Typically, weak bases are loaded into liposomes using ammonium sulfate gradient method, which even significantly improved the pharmacokinetics of the encapsulated drug. However, within a given class of drugs, the drug release rates can vary dramatically. Doxorubicin has an improved toxicity and anti-tumor efficacy profiles. However, another member of the same class, mitoxantrone is released more slowly compared to doxorubicin, its activity is compromised and requires a modified lipid composition to promote release of the drug and improve efficacy. ${ }^{141}$ Weakly acidic drugs can be loaded using reverse $\mathrm{pH}$ gradients (i.e., $\mathrm{pH}$ lower on liposome exterior), including acetate gradient method. ${ }^{142}$ Drugs that contain both weakly basic and weakly acidic groups pose a challenge, but are often overcome depending on the $\mathrm{pKa}$ and number of the various ionizable groups. A drug that contains both a weakly basic amine and a phenolic hydroxyl group, with its relatively high $\mathrm{pKa}$, is still viable for gradient method. The presence of low $\mathrm{pKa}$ acids, make it difficult to find a $\mathrm{pH}$ where both the acid and the amine would be neutralized, enabling the drug to readily penetrate the liposomal membrane and accumulate in the liposome interior. Hydrophobic drugs represent a third class of drugs that are formulated in liposomes. Lipophilic drugs rapidly transfer between liposomal membranes and various biological interfaces, including cellular membranes and plasma proteins. ${ }^{143}$ Therefore, these liposomes often demonstrate some improvement in pharmacokinetic parameters. Highly water soluble molecules include both small molecules and polyionic compounds, such as nucleic acid based therapeutics. Encapsulation of highly water soluble agents may not be amenable to gradient-based drug loading methods due to their minimal membrane permeability. However, nucleic acids, although highly water soluble can be efficiently complexed with cationic lipids to form highly condensed lipidic nanoparticles. ${ }^{144}$ These examples illustrate the barriers and opportunities for delivery of various classes of drugs.

\subsubsection{PEGylation of Liposomes}

Conventional liposomes formed by natural phospholipids and CHOL are generally recognized by the body's immune system as foreign bodies and are often destroyed before significant amounts of drug reach the intended disease site. Liposomes are engulfed by phagocytic cells of the reticular endothelial system (RES), and therefore, drugs accumulate in the liver, spleen or bone marrow. Attachment of ligands, such as polyethylene glycol (PEG) to a liposome surface results in the formation of sterically stabilized liposomes, which prolong drug residence time in blood circulation. ${ }^{145,146}$ This is the patented delivery form, termed stealth ${ }^{\circledR}$ liposomes (ALZA). As shown in Figure 1.4, targeted liposomes usually involve coating with functionalized ligands that bind specifically with receptors on cell surface. The stealth ${ }^{\circledR}$ liposomes maintain chemical stability of the drug in the body and lengthen drug circulation half-life. ${ }^{147,148}$ Therefore, incorporation of steric stabilizing surface functional moiety became an essential component of liposome formulation development. Among the various ligands 


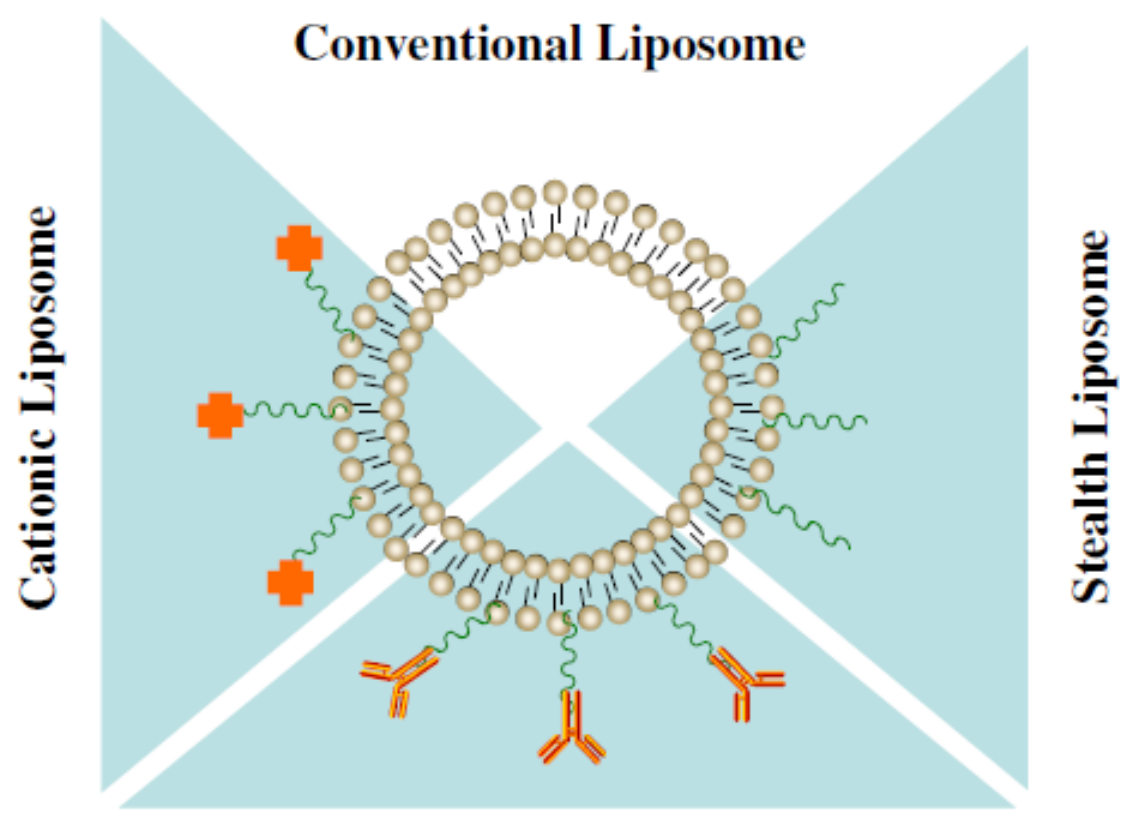

Targeted Liposome

Figure 1.4 Schematic representation of various classes of liposomes.

Conventional liposomes are PEGylated to obtain long circulating liposomes. Conventional liposomes or PEGylated liposomes are surface modified with proteins (e.g. antibodies) to obtain targeted liposomes. Cationic liposomes are obtained by incorporation of cationic lipids (e.g. lipofectamine). 
investigated for improving the blood circulation half-life of liposomes, PEG is the most widely used stabilizer in pharmaceutical applications because of its advantages, including non-biodegradability, high solubility in aqueous solution, ability to bind to a large number of water molecules, high flexibility of its polymer chain, and non-immunogenic and limited accumulation in RES system. ${ }^{21}$ In addition, PEG prevents the adsorption of proteins (opsonins) responsible for phagocytic removal, and thus provides the ability to adjust the liposome physico-chemical (such as drug loading and leakage) and biological properties (e.g. blood circulation and tissue distribution). ${ }^{149,150}$

\subsubsection{Liposome Targeting}

\subsubsection{Passive Targeting}

Targeting of drugs to specific cells and substrates is a critical objective of drug delivery. The size control of liposomes and nature of liposome surface (e.g. surface/steric effect) may be used to optimize passive targeting. ${ }^{122}$ The enhanced accumulation of liposomes at inflammation and tumor sites is due to the leaky vasculature system, ${ }^{151,152}$ which is unable to prevent small species $(<100 \mathrm{~nm})$ crossing the capillary membrane. Even though, only a small fraction of liposomes eventually accumulate at target sites, prolonged circulation indirectly enhances accumulation of liposome associated drugs in the targeted tissues.

\subsubsection{Active Targeting}

Active targeting of liposomes to specific cell receptor sites involves modifying the liposome surface with the incorporation of specific ligands, e.g. peptides, antibodies, immunoglobulins, lectins and other proteins. ${ }^{153}$ A number of key issues must be addressed before active targeting of liposomes using ligand-receptor interactions in vivo. ${ }^{154}$ Targeted liposomes must circulate in blood long enough to perfuse into the target organ and eventually interact with cells. Otherwise, liposomes will be cleared without making contact with the cell targets. ${ }^{155}$ Targeted cell receptor expression should be high compared to non-targeted cells, and receptors must provide sufficient specificity and affinity. Therefore, knowledge of the density of the receptors in target tissue compared with non-targeted sites and the degree of blood perfusion are useful to design targeted liposomes. ${ }^{122}$ In addition, targeting moieties coupled to the surface of liposomes must be sufficiently stable in vivo. In some cases, PEG incorporated into liposomes affects the targeting action and interferes with target (e.g. receptor) recognition. To overcome the barrier of PEG, attachment of targeting ligand to the distal end of a PEG molecule precludes steric hindrance and improves targeting properties of liposomes ${ }^{156,157}$ For example, attachment of a targeting ligand to the maleimide residue of DSPE-PEGmaleimide. ${ }^{119,158}$ The PEG-PE liposomes with targeting moieties retain long survival times in blood circulation and demonstrate target recognition in vivo. ${ }^{159}$ In addition, 
reports suggested that PEG chain length and charge density are crucial for targeted liposomes. ${ }^{119}$

\subsubsection{Methods for Attaching Targeting Ligands to Liposomes}

Over the years, different strategies evolved to impart active targeting to liposomes. All these strategies are based on two chemical methods for attaching ligands to the liposomal surface: covalent and non-covalent coupling. Figure 1.5 describes various covalent reactions used for coupling targeting ligands to liposome surface. Generally, ligands are bound to the surface of liposomes through hydrophobic anchors with functional groups. Long-chain fatty acids, such as palmitic acid ${ }^{160}$ and phospholipids, such as $\mathrm{PE}^{161,162}$ are generally used as anchors. These anchors are incorporated into liposomal bilayers during the formation of liposomes. There are two approaches to covalently attach the ligand to the anchor. The first consists of carrying out the reaction between the ligand and the anchor, and mixing the resulting ligand with other constituents of the liposome. ${ }^{163}$ In the second case, the anchor is already included in the liposome bilayer, and the coupling reaction occurs on the surface of preformed liposomes. ${ }^{164}$

\subsubsection{Coupling of Ligands to the Surface of Liposomes Using thio-ether Bonds}

The reaction between thiol and maleimide groups is an efficient reaction that gives a stable thio-ether bond. Native thiol groups are present in some proteins, but in many others, thiol functions are either absent or present in insufficient amounts. Thiol groups are added either via hetero-bi-functional cross-linking agents ${ }^{165,166}$ or by reducing disulfide bonds. N-hydroxysuccinimide 3-(2-pyridyldithio)propionate (SPDP) and succinimidyl-S-acetylthioacetate (SATA) ${ }^{165,167}$ are commonly used as crosslinkers. Both offer one primary reactive amine residue for coupling with the ligand. In both cases, thiol functions are not directly available, and a deprotection of these functions under mild conditions is necessary before the reaction with liposomes. In the case of SPDP, dithiothreitol (DTT) or an alternative reducing reagent is used to reduce the disulfide bond to thiol function. SATA offers one protected thiol function, which is deacetylated with hydroxylamine. N-(4-(p-Maleimidophenyl)butyryl)phosphatidylethanolamine (MPB-PE) is another commonly used functionalized anchor. ${ }^{168}$ The extended spacer arm between the phospholipid head group and the maleimide moiety reduces the possibility of a steric hindrance at the bilayer interface, and thereby ensures favorable thiol reactivity. Once the liposomes with maleimide groups are formed, the ligands with thiol functions are coupled to the liposomes by simple addition reaction. ${ }^{166,168}$ Now, there is commercial abundance of functionalized lipids with terminal maleimide groups for ease of conjugation with thiol groups of antibodies.

Immunoliposome technology can be combined with sterically stabilized liposomes to give long circulating vesicles capable of delivering antibodies/immunoglobulins to target cells. There are two ways of obtaining immunoliposomes: (I) antibodies are bound to the surface of liposome in parallel with $\mathrm{PEG}^{168}$ and (II) antibodies are linked to the distal end 


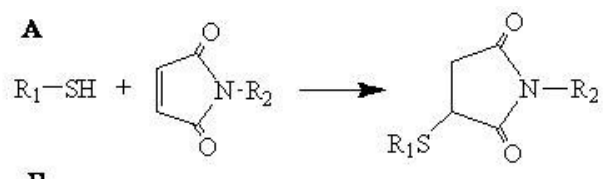

B

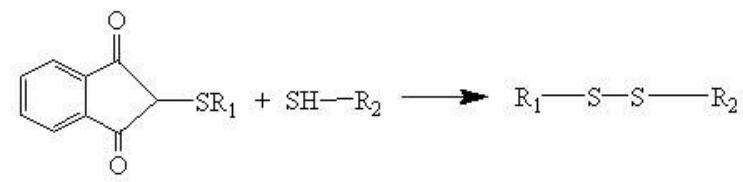

C
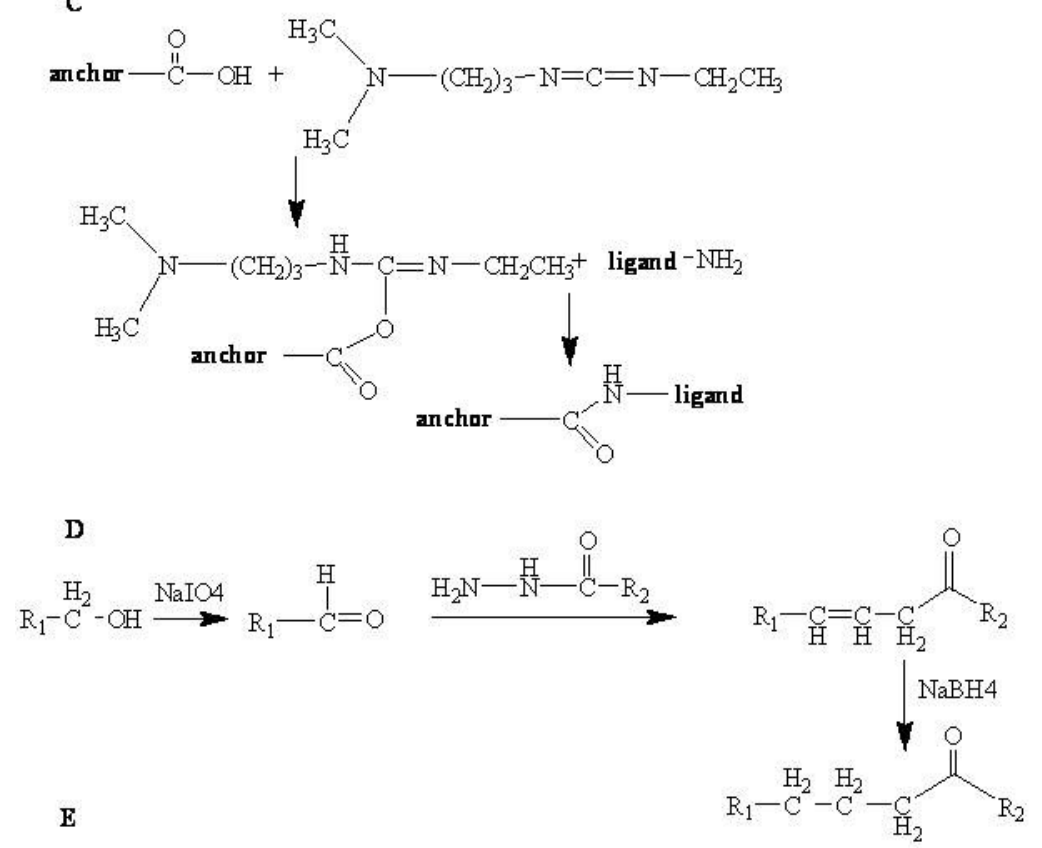

E

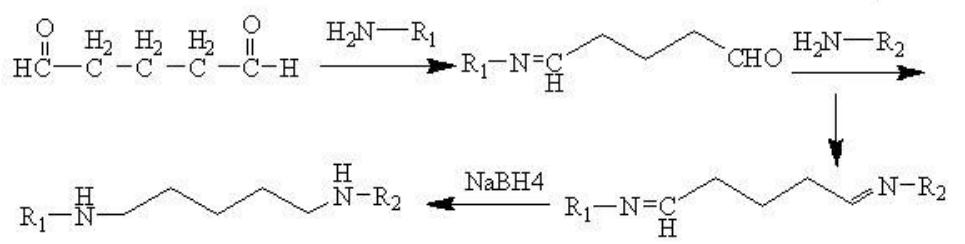

Figure 1.5 Schematic representations of different coupling methods used for surface modification of liposomes.

Reaction between maleimide and thiol functions (A), formation of a disulfide bond (B), reaction between carboxylic acid and primary amine group $(\mathrm{C})$, reaction between hydrazide and aldehyde functions (D), cross-linking between two primary amine functions (E). 
of PEG chains. ${ }^{169}$ For both approaches, PEG is incorporated into the bilayer via an anchor, such as distearoyl phosphatidylethanolamine (DSPE). ${ }^{168,170}$ When antibodies are coupled to the termini of PEG, (maleimidomethyl)cyclohexanecarboxylate-PEG-DSPE (MCC-PEG-DSPE) or maleimido-phenylpropionate-PEG-DSPE (MP-PEG-DSPE) are used. ${ }^{171,172}$ Thiol groups of the Fab ${ }^{s}$ fragments ${ }^{171}$ or monoclonal antibodies (entire IgG) are then attached to the distal end of PEG chains via the maleimide groups. It is also possible to link maleimido antibodies to liposomes offering thiol functions. ${ }^{170,173}$ Antibodies can also be attached either directly to the surface of the vesicles via PDPdioleoyl phosphoethanolamine (PDP-DOPE) ${ }^{170}$ or to the PEG terminus via PDP-PEG$\mathrm{DSPE}^{170}$ or PDP-PEG-PE. ${ }^{169}$ Maleimido antibodies are obtained using a hetero-bifunctional cross-linker, succinimidyl 4-[p-maleimidophenyl] butyrate (SMPB) ${ }^{169,170,173}$ that is reactive with amino and sulfhydryl groups of antibodies. High coupling efficiency is possible when ligands are coupled to the distal ends of PEG chains. ${ }^{170}$

\subsubsection{Attachment of Ligands to Liposomes via a Disulfide Linkage}

One of the most rapid and easy coupling chemistries involves the conjugation of two thiol functions to form a disulfide bond. However, disulfide bonds are relatively unstable under the reductive conditions in serum. ${ }^{174}$ For this reason, the disulfide linkage is progressively replaced by other more stable ones. As seen before, thiolated ligands are generated either by reduction of disulfide bonds ${ }^{174}$ or using SATA or SPDPlinkers. ${ }^{175}$ The thiolated ligands are then made to react with the pyridyldithio moiety of the anchor (PEPDP) to form a disulfide linkage. Several studies demonstrated that the coupling method is efficient in attaching antibody to the liposomes without denaturation of the ligand. ${ }^{175}$

\subsubsection{Cross-linking between Carboxylic Acid Functions and Primary Amines of the Ligand}

Ligands are attached to the surface of liposomes by an amide bond using an anchor functionalized with carboxylic acid end groups. Distearoyl-N-(3-carboxypropionoyl poly(ethylene glycol)succinyl)phosphatidylethanolamine (DSPE-PEG-COOH) offers carboxylic acid groups at the distant end of PEG chains. ${ }^{156,176}$ The coupling reaction is carried out in the presence of N-hydroxysulfosuccinimide (NHS) and 1-ethyl-3-(3dimethylamino-propyl)carbodiimide (EDC) to form an acyl amino ester, which will

subsequently react with the primary amine of the ligand, yielding an amide bond. ${ }^{176,177}$ The advantage of this method is that no prior ligand modification is required, thus reducing the risk of denaturation and loss of specific activity.

\subsubsection{Binding of Ligands to Liposomes via Hydrazone Bond}

Antibodies are covalently bound through their carbohydrate moieties to hydrazide groups grafted onto the liposomal surface to form a hydrazide bond. A mild oxidation of the carbohydrate groups on the constant region of the heavy chain of the immunoglobulin is 
required to produce aldehyde groups. These react with hydrazide groups of the anchor. ${ }^{178}$ The carbohydrate groups are oxidized either by galactose oxidase or by sodium periodate. ${ }^{178}$ Once the oxidized antibodies are formed, they are either directly coupled to the lipid bilayer containing a hydrazide-hydrophobic anchor, such as lauric acid hydrazide ${ }^{178}$ or to the distal end of the PEG chains of sterically stabilized liposomes. ${ }^{170,179}$ In the latter case, a functionalized PEG-lipid hydrazide, Hz-PEG-DSPE ${ }^{180}$ is used. This coupling method is not very efficient because only a small percentage of antibodies $(17 \%)$ were attached to the liposomal membranes. ${ }^{170}$

\subsubsection{Cross-linking between Primary Amines on Liposomes and Primary Amines of the Ligand}

In this method, direct amine-amine cross-linking is performed. Two homo bi-functional cross-linkers are used: glutaraldehyde and suberimidate. ${ }^{181}$ The advantage of this coupling approach is that no prior modification is required to add functional groups to the ligand. The coupling method consists of first activating the primary amine of PE on the liposome surface via the cross-linker and subsequently coupling the ligand. High coupling efficiency ( 60 mole $\%$ ) of antibodies to liposomes was reported. ${ }^{182}$ Despite the fact that the coupling reaction is efficient, the use of homo bi-functional cross-linkers is rarely exploited owing to the uncontrollable homo-polymerization of ligand during the cross-linking reaction.

\subsubsection{Multi-step Attachment Using the Avidin-Biotin Interaction}

The avidin-biotin strategy is a popular and versatile tool for active targeting. ${ }^{157,183,184}$ Anita et al demonstrated the use of a biotinylated PEG-DSPE linker to form a noncovalent bond between streptavidin-conjugated monoclonal antibody (mAb) (e.g. OX26 $\mathrm{mAb}$ raised against the rat transferrin receptor) and liposomes. ${ }^{184}$ Furthermore, biotinylated antibodies are easily obtained. ${ }^{185,186}$ Huwyler et al demonstrated specific targeting of daunomycin to the rat brain using OX26 antibody conjugated liposomes. ${ }^{157}$

\subsubsection{Non-covalent Methods}

An alternative means to conjugate ligands to liposomes is the use of non-covalent methods. The advantage of these methods is easy to be carried out without the need of aggressive reagents. A simple method is to merely add the ligand to the mixture of phospholipids during the preparation of the liposomes. However, the percentage of ligand attached to the carrier is relatively low (4-40\%) and aggregation of the liposomes is frequently observed. Furthermore, the amount of ligand linked to the liposome is not easily controllable and the correct orientation of the antibodies is not ensured. Finally, detachment of the antibody in vivo might occur. 


\subsubsection{Preparation of Liposomes}

\subsubsection{Passive Loading}

Over the last 60 years, numerous methods are reported for the preparation of liposomes. On a small scale, Bangham method is the commonly employed method. In this method the lipid film is hydrated with drug solution/suspension to encapsulate or incorporate drug moieties. ${ }^{154}$ Usually, hydrophilic drugs should have a large encapsulated volume. On the other hand, the encapsulation efficiency of hydrophobic drugs depends on the quantity and quality of lipids. ${ }^{187,188}$ In an appropriate preparation method, concentration of lipids and drugs, drug/lipid ratio, presence of functionalized lipids, $\mathrm{pH}$, buffer and process parameters play a critical role in the encapsulation efficiency of drugs. ${ }^{122}$

\subsubsection{Active Loading: Gradient Loading Strategies}

The drug encapsulation method is important in determining the in vivo stability of liposomes. Hydrophobic drugs are usually encapsulated passively by hydration of the lipid film with drug solution. ${ }^{189,190}$ Some therapeutic agents, including small molecules and proteins can be complexed with charged lipid components. ${ }^{144,191}$ However, one of the most widely used encapsulation methods employs transmembrane gradients to efficiently load and subsequently stabilize weakly basic amphipathic drugs inside the core of liposomes. These include: (I) simple $\mathrm{pH}$ gradients using citric acid solutions, ${ }^{192,193}$ (II) ammonium ion gradients employing citrate ${ }^{194}$ or sulfate, ${ }^{195}$ (III) alkyl, ${ }^{196}$ (di-alkyl) or trialkyl ammonium salts, ${ }^{197}$ (IV) transition metal concentration gradients $\left(\mathrm{Cu}^{2+}, \mathrm{Mn}^{2+}\right.$, $\mathrm{Zn}^{2+}$ and $\left.\mathrm{Mg}^{2+}\right)^{198-200}$ and (V) transmembrane gradients of drug solubility. ${ }^{201}$ The cation entrapped in the liposome interior plays a role either in establishing a $\mathrm{pH}$ gradient across the membrane that drives the accumulation of weakly basic drugs into the liposome interior, or directly exchanging with the drug molecule. The counter ion plays an important role in stabilizing the formulation to premature leakage of encapsulated drugs. Figure 1.6 depicts an example of active loading and subsequent stabilization process of doxorubicin using ammonium sulfate gradient method. ${ }^{202}$

Liposomal formulation of the camptothecin prodrug, irinotecan, displayed a different pharmacokinetic behavior in lieu of the encapsulation method. ${ }^{203}$ Using manganese sulfate gradient method, Bally et al. ${ }^{203}$ prepared irinotecan liposome formulation, whose drug release half-life improved from $8.3 \mathrm{~h}$ to $56.8 \mathrm{~h} .{ }^{197}$ The intraliposomal drug concentration also affects the stability of liposomal drugs prepared using gradient loading methods. At low drug/lipid ratio, doxorubicin was retained in the liposomes due to the strength of the residual gradient in the carrier. ${ }^{203}$ A high drug/lipid ratio resulted in the exhaustion of $\mathrm{pH}$-gradient across the membrane leading to an increased leakage of the drug. Similarly, studies with high ionic concentration gradient using ammonium sulfate improved doxorubicin formulation stability. ${ }^{202}$ Insoluble drug precipitates formed due to the complexation of drug and intraliposomal ions may acquire desirable zero-order release rates. This could be due to the fact that the dissolved drug maintains constant 


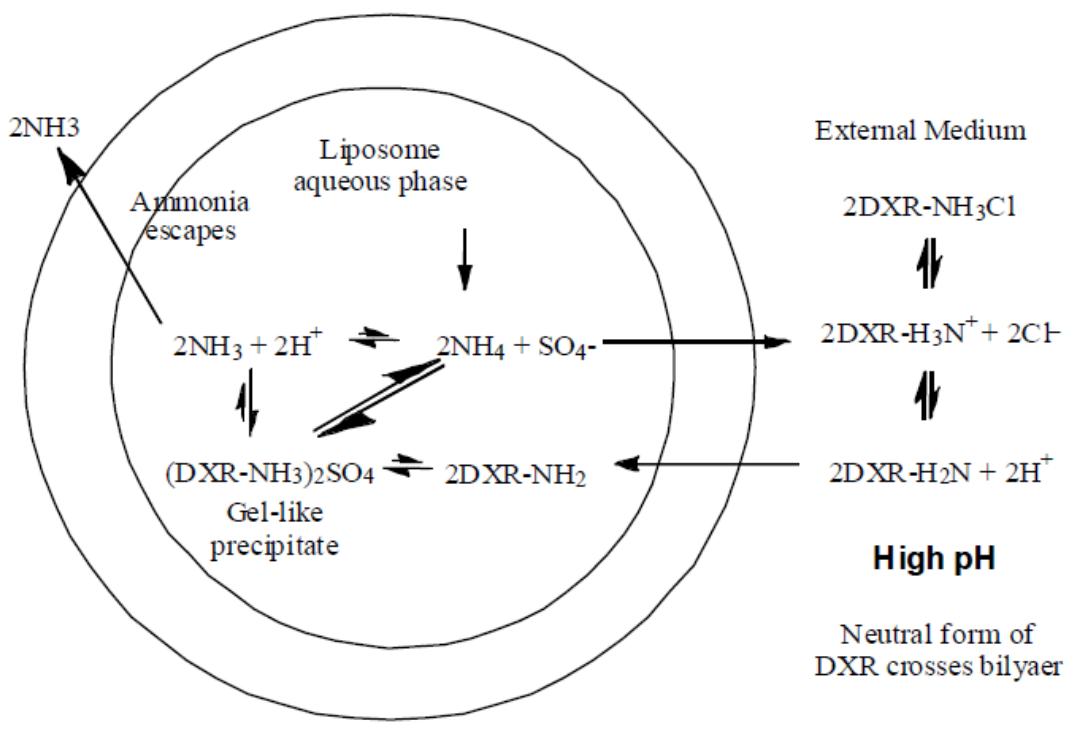

A. DOXIL loading by ammonium ion gradient m ethod

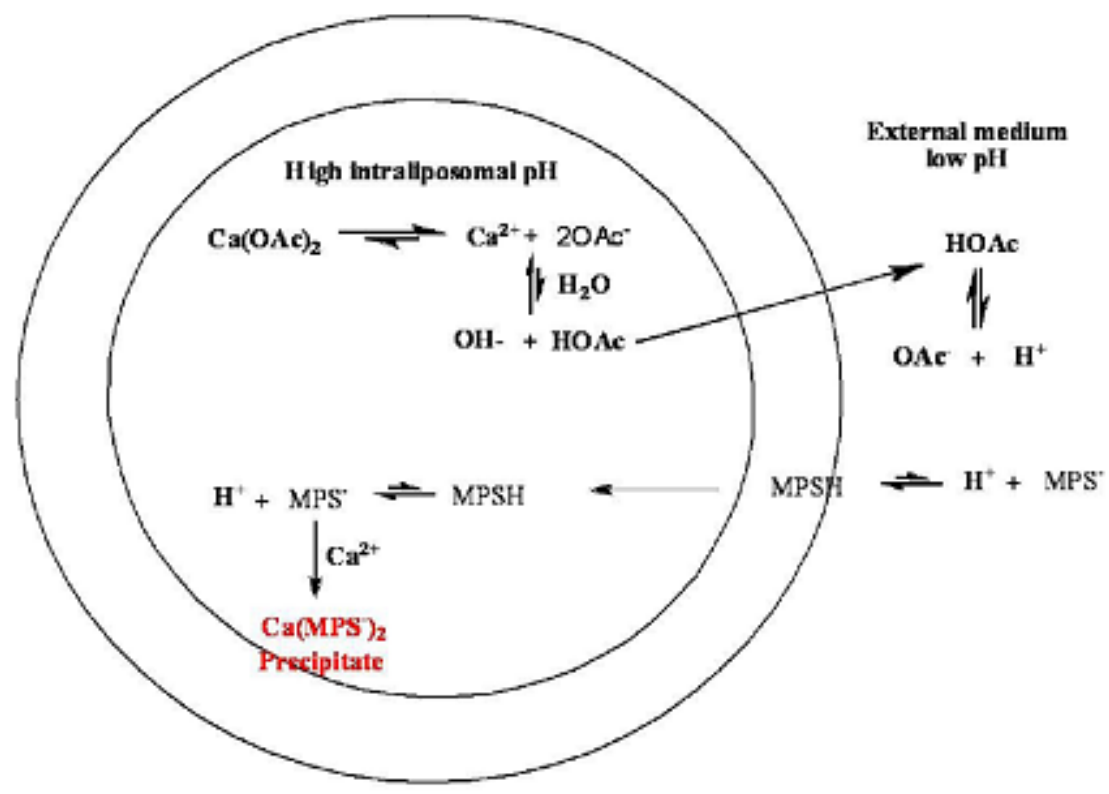

B. MPS active loading by calcium acetate gradient method

Figure 1.6 Schematic representation of the liposomal loading of weak bases (A) and weak acids (B).

In the former case, liposomal aqueous core is acidic, which causes the formation of a gellike precipitate of DXR. In the latter case, unionized MPS diffuses across the lipid bilayers and forms an insoluble calcium-MPS complex inside the liposome core. 
equilibrium with the precipitated drug fraction of the liposomes. ${ }^{204}$ In addition, the potency of the drug determines the selection of drug/lipid ratios for the preparation of liposomes. Many liposome formulations display dose-dependent pharmacokinetics; therefore, a high drug/lipid ratio will result in low lipid doses, and thus may contribute to rapid clearance of liposomes. Even pegylated liposomes are vulnerable for fast clearance from the body. Therefore, high drug/lipid ratios may be viable for low to moderate potency drugs, such as the prodrugs, ${ }^{197}$ but not for potent drugs.

Low concentration of anionic lipids was shown to enhance the stability of encapsulated drugs using transmembrane electrochemical gradient. ${ }^{205,206}$ Fluoxuridine retention increased upon inclusion of only $20 \mathrm{~mole} \%$ of distearoyl phosphatidylglycerol (DSPG) in DSPC liposomes, and in a separate study, the inclusion of $15-55$ mole $\%$ of DSPG in liposomes improved the retention of vincristine using a methyl ammonium gradient. ${ }^{206}$ It was hypothesized that the inclusion of negatively charged lipids increased the membrane partition coefficient of weakly basic drugs and improved their retention in liposomes maintaining a transmembrane $\mathrm{pH}$-gradient. However, the presence of a negative charge at the membrane interface in other formulations caused leakage of encapsulated drug. ${ }^{207}$ Therefore, physico-chemical properties of drugs and phospholipids play a critical role in the selection of active loading strategy for encapsulation of drugs.

\subsubsection{Liposome In Vivo Kinetics: Systemic Administration and Aerosolization}

The rate of in vivo drug release is an extremely important parameter since it influences the rate of clearance of the drug from the general circulation, bioavailability, and activity of the drug at its site of action and targeting ability of the drug. ${ }^{122}$ In vivo encapsulated drug concentration can be determined either directly by measuring purified liposomal drug in plasma ${ }^{197,208}$ or indirectly by assuming that the clearance rate of the unencapsulated drug is sufficiently faster than the clearance rate of the liposomes (by simply following changes in the plasma drug-to-lipid ratios). ${ }^{197}$ However, challenges pose for hydrophobic drugs that demonstrate significant protein binding. For some drugs, the rate of in vivo drug release can be controlled by manipulation of physico-chemical properties of the liposomes.

Different applications may require distinct release kinetics and different particles sizes. ${ }^{209}$ Following an intravenous injection, the first capillaries to be encountered by a delivery vehicle are in the lung tissue, which tends to mechanically entrap large particles, such as multilamellar vesicles (MLVs). The lung thus represents a unique site for drug delivery if large liposomes are employed, but care has to be taken to avoid pulmonary emboli. Administration of liposomal formulations to the epithelial cells in the lower airways of the lung by nebulization requires smaller particles $(\sim 300 \mathrm{~nm})$ that are in the respirable range. ${ }^{210}$ To deliver imaging agents, a rapid burst will achieve maximum contrast between the circulation system and the target tissues.

The "first pass" organ to be encountered after systemic administration is the liver, which can thus be targeted with fast-release liposomes. Large vesicles are generally more 
rapidly eliminated from the bloodstream, whereas small ones circulate for longer, especially those with neutral or positively charged, rigid bilayers. Liver, lung and spleen may be circumvented using stealth liposomes. Usually, liposomes do not pass through the endothelial barrier of the vascular system; however, under pathological conditions, small particles $(<100 \mathrm{~nm})$ are able to extravasate through the poorly formed neovasculature of some solid tumors. ${ }^{211}$ Regardless of the circulation time of an intravenously injected liposomal dose, the majority will eventually be taken up by the mononuclear phagocyte system. Using alternate parenteral routes, such as intraperitoneal, subcutaneous or intramuscular, large liposomes generally tend to be retained longer at the site of injection, while small ones are mainly taken up by the draining lymph system.

Aerosolized liposomal preparations became a feasible route for delivering drugs to the lung. A combined aerosol of liposomal paclitaxel and cyclosporin A achieved better results in the treatment of pulmonary metastases of renal-cell carcinoma. ${ }^{212}$ Spray-dried liposome powder formulations were used as carriers for superoxide dismutase (SOD). ${ }^{213}$ Improved delivery of rifampicin by aerosolized liposomes to alveolar macrophages might become significant in the treatment of tuberculosis. ${ }^{214}$ Aerosolized liposomal budesonide is effective against experimental asthma in mice. ${ }^{215}$ Aerosol formulations of liposomal 9nitrocamptothecin are non-toxic, and efficiently treated melanoma and lung metastases in mice. ${ }^{216}$ Liposomal paclitaxel in aerosol effectively treated pulmonary metastases in a murine renal carcinoma model. ${ }^{217}$ Nebulization was recently proposed as a means to deliver liposomal aerosols. ${ }^{218}$

\subsubsection{Liposomes: Clinical and Diagnostic Applications}

Recent advances in liposome technology have led to the development of "stealth liposomes" whose prolonged circulation lifetime improved the therapeutic benefits. For cancer and inflammation diseases, therapeutic potential could be improved by receptor specific targeting and cell specific delivery of therapeutic moieties. Despite some progress, one of the current challenges is the targeting specificity and affinity of the binding motif to the disease sites. In an ideal targeted delivery system, high binding specificity avoids adverse effects to the surrounding tissues within the vessel and/or downstream of the injury site, while high binding affinity enables competition with natural ligands. As targeted liposomal drug delivery systems are now becoming feasible, it offers significant potential for local delivery of anti-cancer, anti-inflammatory, anticoagulants.

In addition to clinical applications, liposome formulations are used for as contrast agents for experimental diagnostic imaging of liver, spleen, brain, cardio-vascular system, tumours, inflammation and infections. Gamma-scintigraphy, near infrared fluorescence imaging (NIRF) and magnetic resonance imaging (MRI) require a sufficient quantity of radionuclide or fluorescent dye or paramagnetic metal to be associated with the liposome. There are two possible routes to improve the efficacy of liposomes as contrast mediums for gamma-scintigraphy or MRI or NIRF: I) increasing the quantity of carrier-associated marker and II) enhancing the signal intensity. To increase the load of liposomes with 
reporter metals, amphiphilic chelating polymers, such as N, $\alpha$-(DTPA-polylysyl)glutaryl phosphatidyl ethanolamine were introduced. These polymers easily incorporate into the liposomal membrane and markedly increase the number of chelated $\mathrm{Gd}$ or In atoms attached to a single lipid anchor. In the case of NIRF, fluorescent dyes are incorporated into the lipid bilayers of liposomes. Imaging approach using contrast liposomes allows the visualization of pathological areas. Liposomes loaded with imaging agents are also used for the in vivo monitoring of tissue pharmacokinetics of liposomal drugs.

\subsubsection{Stability of Liposomes}

The shelf-life of liposomes is determined by physical and chemical aspects of liposomes. ${ }^{122}$ Physical processes, such as aggregation and fusion result in loss of liposome encapsulated drug and changes in size. In principle, this process is reversible; however, fusion of liposomes further triggers coalescence of liposomes. As coalescence is an irreversible process, liposomes cannot be retrieved.

\subsubsection{Physical Stability}

Stable liposome suspensions require a repulsive interaction that is at least comparable to the magnitude and range of vander Waals forces. ${ }^{219,220}$ Repulsive interactions at the surface of liposomes are a function of: I) electric potential (zeta potential, $\zeta$ ) at the plane of shear and II) surface charge density $(\sigma)$. The zeta potential is calculated from electrophoretic mobility by Equation 1.1 $\mathbf{1}^{154,221}$

$$
\zeta=\eta / \varepsilon_{0} \varepsilon_{\mathrm{r}}
$$

Equation 1.1

The surface charge density calculation is based on Gouy-Chapman double layer theory. ${ }^{222-225}$ The theory is applicable to unabsorbed particle surfaces. However, ions adsorb on the surface of liposomes giving rise to a Stern layer so that the double layers must be described by the Gouy-Chapman-Stern theory (Figure 1.7). ${ }^{225}$ Later on, the problems associated with the distortion of the electrical double layers due to the motion of the charged liposomes (e.g. PE/PEG-PE liposomes) and the influence of surface conductivity on electrophoretic mobility superceded the Gouy-Chapman-Stern theory with Derjaguin-Landau-Verwey-Overbeek (DLVO) theory.

Jones $^{223}$ stated that the classic DLVO model is applicable for determining the stability of liposomes, considering that many of the physico-chemical properties of liposomes resemble that of conventional colloidal particles. The total interaction potential is a function of the repulsive and the attractive components. The short range attractive forces between liposomes are of van der Waals forces, while the long range repulsive forces are electrostatic repulsive forces. These forces are calculated using the Poisson-Boltzmann equation. The repulsive force arising from the overlap of the electrical double layers surrounding charged liposomes is the main reason for the stability of liposome dispersion. ${ }^{226}$ The electrical forces increase exponentially as liposomes approach one 


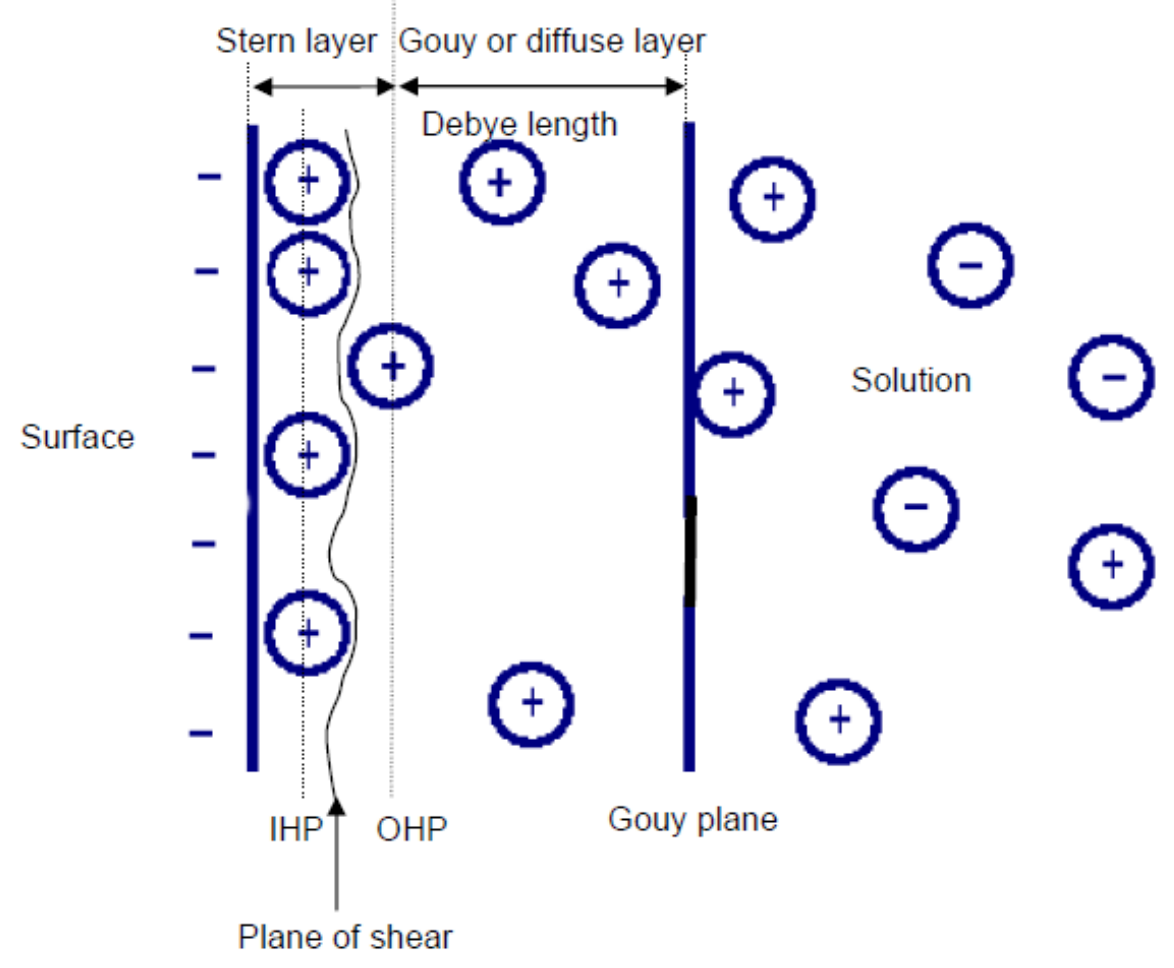

Figure 1.7 Schematic diagram of the Gouy-Chapman-Stern diffuse double layer theory.

The theory states that ions do have finite size so cannot approach the surface closer than a few nm. The first ions of the Gouy-Chapman diffuse double layer are not at the surface, but at some distance away from the surface. This distance will usually be taken as the radius of the ion. According to Stern, it is possible that some of the ions are specifically adsorbed by the surface in the plane of shear and this layer has become known as the Stern Layer. The potential at the OHP is considered as the zeta potential. The double layer is formed in order to neutralize the charged surface, and in turn causes an electrokinetic potential between the surface and any point in the mass of the suspending liquid. This voltage difference is on the order of millivolts, and is referred to as the surface potential. The magnitude of the surface potential is related to the surface charge and the thickness of the double layer. From the surface, the potential drops off linearly in the Stern layer and then exponentially through the diffuse layer, approaching zero at the imaginary boundary of the double layer. The potential curve indicates the strength of the electrical force between the particles and the distance. The particle (liposome) mobility is related to the dielectric constant and viscosity of the suspending liquid, and to the electrical potential at the boundary between the moving particle and the liquid.

Adapted with permission. Jones, M.N. The surface properties of phospholipid liposome systems and their characterisation. Adv Colloid Interface Sci 54, 93-128 (1995). ${ }^{223}$ 
another, and the attractive forces increase as an inverse power of separation. Other forces also contribute to the interaction between liposomes. Addition of other interactions expands the DLVO model to an extended DLVO interparticle model. According to the extended DLVO theory, the total force $\left(\mathrm{F}_{\mathrm{T}}\right)$ between the liposomes is given by Equation 1.2:

$$
\mathrm{F}_{\mathrm{T}}=\mathrm{F}_{\mathrm{vdw}}-\mathrm{F}_{\text {hfo }}+\mathrm{F}_{\text {est }}+\mathrm{F}_{\text {und }}+\mathrm{F}_{\text {hgd }}+\mathrm{F}_{\text {steric }} \quad \text { Equation } 1.2
$$

As described previously, $\mathrm{F}_{\mathrm{vdw}}$ and $\mathrm{F}_{\text {est }}$ represent van der Waals and double layer repulsive forces between colloidal particles. ${ }^{225}$ For neutral liposomes e.g. EPC liposomes, the bilayers can approach within the order of 5-20 nm, and under these conditions, the repulsive hydration force $\left(\mathrm{F}_{\mathrm{hgd}}\right)$ is the major repulsive interaction between lipid bilayers. At small separations, a repulsive interaction may arise from out of plane motions or undulations which give rise to an undulation force $\left(F_{u n d}\right) . F_{\text {und }}$ is only of importance for fluid bilayers, e.g. egg lecithin bilayers account for approximately $25 \%$ of the van der Waals forces. ${ }^{227}$ The hydrophobic force $\left(\mathrm{F}_{\mathrm{hfo}}\right)$ is a long-range force and operates at a greater distance than $\mathrm{F}_{\mathrm{vdw}}{ }^{228}$

The electrophoretic mobility of PEGylated liposomes is very low when compared to nonpegylated liposomes, and the electrophoretic mobility show little variation with DSPEPEG levels of liposomes in the range 5-10 mole $\%$. The inclusion of CHOL has little or no effect on the mobility. The low electrophoretic mobility corresponds to low zeta potentials. For PEGylated liposomes, a steric force will arise when the surface polymer chains interact. Several theoretical approaches to the interaction between surfaces with adsorbed polymers were proposed..$^{229-231}$ There are two limiting cases of repulsive forces; at low polymer density where the polymer separation exceeds the polymer coil size ('mushroom model'), and at high polymer density when the polymer chains take up extended conformations ('brush model'). It is noted that bridging between two polymer coated surfaces involves polymer interdiffusion and entanglements that lead to attractive interaction. ${ }^{232-234}$ Needham et al. ${ }^{235}$ reported that steric stabilization is a result of the combination of electrostatic and steric forces.

The electrostatic component results from a net charge on the liposome surface and/or the charge associated with the adsorbed polymer (e.g. PEG chains). And the steric component arises from the chain-chain exclusion of adsorbed polymeric material on one surface from the other surface. The coalescence kinetics in colloidal systems was performed by von Smoluchowski. ${ }^{236,237}$ The total number of vesicles at time t, $\mathrm{N}$ may be approximated by Equation 1.3:

$$
\mathrm{N}=6 \varphi / \pi \mathrm{d}^{3} \quad \text { Equation } 1.3
$$

Where $\mathrm{d}$ is vesicle diameter and $\varphi$ is the liposome volume fraction. For liposome systems, the entrapped aqueous volume is estimated by $\mathrm{N}_{\mathrm{VOL}}$ method. ${ }^{238}$ The aggregation process in colloidal systems is a second-order process. Mean diameter $\left(\mathrm{D}_{\mathrm{t}}\right)$ of liposomes at time, $t$ is determined from Equation 1.4: 


$$
D_{t}=\left[d^{3}+(6 k \varphi / \pi) t\right]^{1 / 3}
$$

\section{Equation 1.4}

Where $\mathrm{d}_{0}$ is the initial vesicle diameter at $\mathrm{t}=0$ and $\mathrm{D}_{\mathrm{t}}$ is the liposome diameter at time $\mathrm{t}$. From DLS studies, mean liposome diameters can be estimated. By plotting $\mathrm{d}^{3}$ versus time, we can calculate the rate constant for aggregation, $\mathrm{k}=6 \mathrm{k} \varphi / \pi$.

\subsubsection{Chemical Stability}

As phospholipids form the backbone of the bilayer, their chemical stability is important. Two types of chemical degradation reactions affect the performance of phospholipid bilayers: hydrolysis of the ester bonds linking the fatty acids to the glycerol backbone and peroxidation of unsaturated acyl chains. The oxidation and hydrolysis of lipids may lead to the appearance of short-chain lipids, and then soluble derivations will form in the membrane resulting in the decrease of the quality of liposome products. The chemical stability of liposomes is improved by inclusion of anti-oxidants, such as $\alpha$-tocopherol and saturated phospholipids.

\subsubsection{Lyophilization: Stabilization of Liposomes}

Lyophilization of liposomes can prevent hydrolysis of the phospholipids and physical degradation of liposomes during storage. In addition, it may stabilize the particle size and leakage of drugs encapsulated inside the liposomes. Lyophilization of liposomes results in an elegant dry cake, which can be reconstituted to obtain the original liposomal suspension. On the other hand, the lyophilization process may induce physical changes in liposomes, such as leakage of the encapsulated drug and changes in the vesicle size. The interaction between the hydrophilic phospholipid head groups and water molecules plays a key role in the formation of liposomal bilayers. Thus, removing water from the liposomes by lyophilization presents a challenge. Evidence suggests that excipients, such as disaccharides (e.g. sucrose and trehalose) protect the liposomes during the lyophilization process (lyoprotectants). ${ }^{239}$ As illustrated in Figure 1.8, lyophilization process is comprised of three phases: (I) freezing cycle, (II) primary drying and (III) secondary drying. The parameters of each phase depend on the selected formulation. In the freezing phase, buffer components and lyoprotectants form an amorphous matrix in and around the liposomes that prevents fusion, and protects the liposomes against rupture due to the growth of ice crystals. As the temperature of the frozen matrix is lowered, the viscosity of the frozen matrix increases, until it forms a glass with low molecular mobility. The temperature at which this occurs is called Tg', glass transition temperature of the frozen matrix. The $\mathrm{Tg}$ ' is a function of frozen matrix composition, but liposomes do not contribute to the value of $\mathrm{Tg},{ }^{241,242}$ The freezing cycle carried at a slow rate allows formation of pores and facilitates sublimation during primary drying phase. This in turn minimizes the leakage of contents from liposomes after rehydration of the lyophilized sample. Another option to increase water evaporation at the sublimation front is annealing process. 


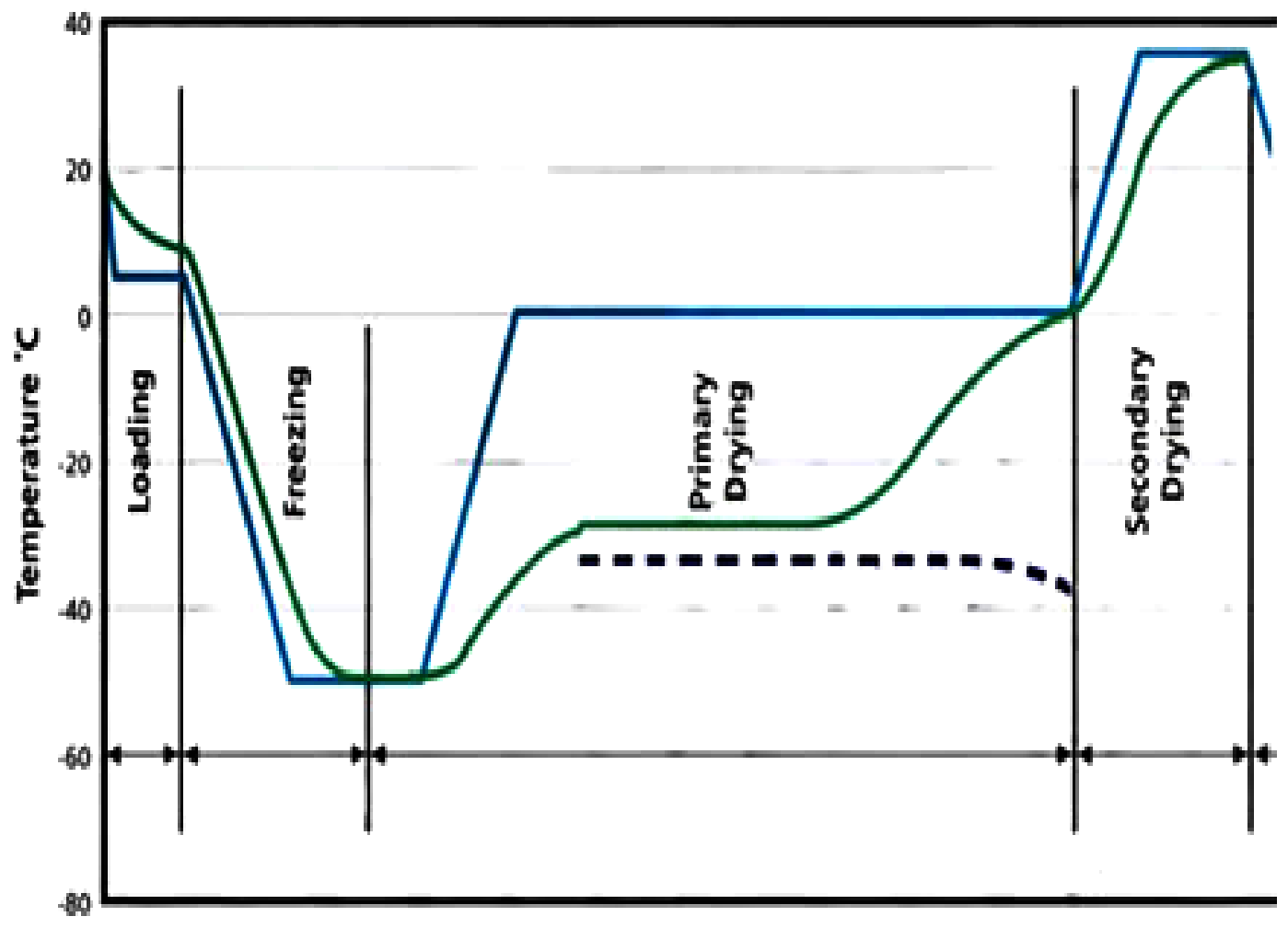

Figure 1.8 Schematic representation of different stages of a lyophilization cycle.

It describes the relation between the product's (green solid line)/shelf (blue solid line) temperatures to the chamber pressure inside the lyophilizer. The product is frozen below the glass transition temperature of the amorphous product matrix ( ${ }^{\prime}$ '). Primary drying is also carried below $\mathrm{Tg}$ ' to avoid product cake collapse and crystallization of the product matrix. Secondary drying is performed to remove residual moisture of the product. Vacuum during the primary drying is usually 100-300 mTorr and 50 mTorr in secondary stage.

Adapted with permission. Gea, G.H. Thermodynamic Lyophilization Control-TLC. Process Analytical Technology 12, 1-9 (2008). ${ }^{240}$ 
Crystallization of the ice can be optimized further by "annealing" of the frozen samples: the samples are heated to a temperature above Tg' and are cooled again before the start of the lyophilization process. The energy that becomes available for the frozen matrix may induce further crystallization of unfrozen water in the amorphous matrix ("devitrification"). In addition, small crystals may re-crystallize into larger ones. The result of annealing is often an increase in sublimation rate during primary drying. ${ }^{243} \mathrm{In}$ addition, liposomes may be better protected against lyophilization stress. The effect of annealing may depend on the specific formulation (e.g., excipients, lipid composition, and vesicle size). ${ }^{244}$

During primary drying, the ice crystals are sublimated, resulting in the porous cake structure of the freeze-concentrated matrix. The temperature at the sublimation front is maintained $5^{\circ} \mathrm{C}$ below $\mathrm{Tg}$ '. The product temperature at the sublimation front is determined by local pressure, according to the ice vapor pressure-temperature. The set point of the chamber pressure is chosen at a value that corresponds to an ice temperature of about $4^{\circ} \mathrm{C}$ below Tg'. The shelf temperature should be higher than the ice temperature in order to transfer the heat to the sublimation front for drying. The driving force of the drying process is the difference in temperature, and therefore, difference in water vapor pressure between the condenser of the lyophilizer and the sublimation front. Heat transfer from the shelf to the sublimation front occurs via conduction by glass and ice as well as by gas molecules. ${ }^{245}$ In conclusion, careful control of chamber pressure and shelf temperature as well as knowledge of the sample $\mathrm{Tg}$ ' are prerequisites for successful lyophilization.

In the secondary drying phase, the residual water content of the amorphous matrix is further decreased. Followed by the primary drying phase, when the product temperature approaches the shelf temperature, the secondary drying phase can be initiated. In the secondary drying phase, the pressure is reduced to a minimal value and the shelf temperature can be gradually increased. Reduction of the water content of the cake occurs during primary drying, but the cake is further dried to $<1 \% \mathrm{w} / \mathrm{w}$ of the total moisture content. Reduction of the water content increases the glass transition temperature ( $\mathrm{Tg}$ ) of the cake, and this allows for a gradual increase in shelf temperature. The shelf temperature is a key parameter for obtaining low residual water content. Vials can be closed under vacuum or after (partially) releasing the vacuum with nitrogen gas or argon.

The optimal liposome size for high retention of a water soluble compound after freezedrying is $0.1-0.2 \mu \mathrm{m} .{ }^{246}$ Rigid liposomes are more vulnerable to high cooling rates $\left(0.5^{\circ} \mathrm{C} / \mathrm{min}\right)$ than liposomes with flexible bilayers, but give a high retention $(70-90 \%$ $\mathrm{w} / \mathrm{v}$ ) of the encapsulated hydrophilic compounds. Rigid liposomes are usually composed of saturated phospholipids. Addition of CHOL (e.g. $30 \mathrm{~mole} \%$ of the total lipid content) may increase the resistance of liposomal bilayers to lyophilization stress. Lyoprotectants form an amorphous, glassy matrix in and around the liposomes. Interaction between the lyoprotectant and the phospholipid head groups is important for preventing leakage during rehydration of liposomes. ${ }^{247}$ Recently, a different mechanism ${ }^{247-249}$ was proposed by which sugars may reduce the stress on bilayers during drying. Bilayers dried without lyoprotectant approach each other and the suction of water by the phospholipid causes a 
lateral compressive stress. The mere presence of a glass between adjacent bilayers during drying provides spacing between the bilayers, and in combination with the attraction of water by the sugars (osmotic properties), the compressive stress on the bilayers is reduced. The preferred lyoprotectants are disaccharides, such as sucrose and trehalose. Trehalose has a high $\operatorname{Tg}\left(100-115^{\circ} \mathrm{C}\right)$ in the dried state $(<0.5 \% \mathrm{w} / \mathrm{w}$ residual water $)$ than sucrose $\left(70^{\circ} \mathrm{C}\right)$, which may be an advantage for the storage stability of the dried product. Lyoprotectants provide optimal protection of the liposomes if present at high sugar/phospholipid ratios. In addition, the lyoprotectant should be present on both inside and outside the liposomes. ${ }^{250} \mathrm{~A}$ total solute concentration of more than $30 \% \mathrm{w} / \mathrm{v}$ may hinder the drying process. The use of crystallizing excipients, such as glycine and mannitol is not recommended for the lyophilization of liposomes. ${ }^{251}$ A crystalline excipient can facilitate the primary drying process and improve the appearance of the freeze-dried cake. However, the successful use of crystalline excipients for the lyophilization of liposomes is not reported so far, and the lack of an amorphous phase in and around the liposomes may damage the bilayers. The use of low molecular weight excipients, such as sodium chloride, glucose and amino acids should be minimized. Such excipients lower the Tg' of the freeze-concentrate and further, the Tg of the lyophilized cake. A low Tg' requires lyophilization at a low product temperature, which is an inefficient process. A decrease in product temperature of $5^{\circ} \mathrm{C}$ may double the primary drying duration.

An acceptable shelf-life is a pre-requisite for successful introduction of liposome formulations into therapy. With advances in formulation and lyophilization process research, the shelf-life of the specialized targeted liposomes could be improved.

\subsection{Summary and Rationale}

Pulmonary edema, pro-inflammatory mediators' production and leukocyte migration are the hallmark features of acute lung injury. In the current investigation, human ALI features were mimed in IL-1 $\beta$ stimulated rat model. Pulmonary edema and leukocyte migration are mediated via $\alpha \mathrm{v} \beta 6$ integrin and ICAM-1 receptors upregulated on alveolar epithelium or endothelium, respectively. This poses a serious challenge to find a common ground for targeting to each of the receptors. Citing a spatial and disparate expression of receptors, the current problem was addressed using different targeting motifs administered by nebulization and systemic routes of administration. In response to IL- $1 \beta$ stimulation, $\alpha v \beta 6$ integrins of alveolar epithelium interact with RGD domain of the LAPTGF- $\beta$ complex inducing an increase in permeability across alveolar epithelium. Therefore, RGD sequence was identified as the targeting motif to intervene the interaction of $\alpha v \beta 6$ integrins with LAP-TGF- $\beta$ complex.

Our first strategy is to utilize the cRGD-peptide as the targeting motif on the liposome surface. Due to the apical expression of $\alpha v \beta 6$ integrins on the alveolar epithelium, MPS$\mathrm{L}^{\mathrm{CRGD}}$ were nebulized to exert direct impact on epithelium for inhibition of pulmonary edema. Also, ICAM-1 receptors are upregulated on alveolar endothelium to aid in the neutrophil migration into alveoli during ALI. An anti-ICAM-1 antibody might disrupt the 
interaction of ICAM-1 receptors with $\beta 2$ integrins of neutrophils, and thus impedes neutrophil migration. Our second strategy is to conjugate antibody on the MPS encapsulated liposomes to target ICAM-1 receptors, which are accessible by systemic administration. Both the strategies deliver MPS to the targeted tissues for inhibition of pro-inflammatory cytokine and chemokine production. Each of these strategies has the potential to modulate ALI pathogenesis. The success of the strategies depends on the answers of the following central questions: (I) how efficient are the conjugation reactions of MPS-L ${ }^{\text {cRGD }}$ and MPS-L ${ }^{\mathrm{Ab}}$, (II) how efficient are the MPS-L $\mathrm{L}^{\mathrm{cRGD}}$ and MPS-L ${ }^{\mathrm{Ab}}$ to interact with their counter receptors, (III) how efficient are the MPS-L ${ }^{\mathrm{cRGD}}$ and MPS-L ${ }^{\mathrm{Ab}}$ to internalize in the targeted cells and (IV) how stable are the MPS-L ${ }^{\text {cRGD }}$ and MPS-L ${ }^{\mathrm{Ab}}$ in vitro and in vivo. Through addressal of each of these critical issues, the underlying ALI pathogenesis can be resolved. 


\section{CHAPTER 2. CENTRAL HYPOTHESIS AND SPECIFIC AIMS}

\subsection{Central Hypothesis}

The overall goal of the proposed research is to reduce inflammation associated with ALI. To achieve the goal, I propose to design new liposome drug delivery systems that selectively target and deliver methylprednisolone succinate to the inflamed alveolar epithelium and endothelium. It is well accepted that RGD domain and ICAM-1 receptors play a critical role in mediating pulmonary edema and neutrophil migration respectively. Based on this, the proposed research is focused on the design and development of liposomes that utilize the cRGD-peptide and anti-ICAM-1 antibody tethered to the liposome surface as the targeting motifs. The proposed research is based on the central hypothesis that "methylprednisolone succinate encapsulated targeted liposomes bind to integrin or ICAM-1 receptors and modulate ALI pathogenesis".

Completion of these studies provided a promising drug delivery system for receptor mediated inflammatory diseases. Therefore, in order to test the hypothesis, I propose the following specific aims:

\subsection{Specific Aims}

\subsubsection{To Develop MPS Encapsulated Targeted Liposomes}

The purpose of this specific aim was to prepare MPS encapsulated liposomes, surface modified by cRGD-peptide and anti-ICAM-1 antibody. In the former case, (DSPEPEG2000-maleimide)-cRGD-peptide conjugate was synthesized by Michael addition reaction and the conjugate was incorporated into MPS encapsulated liposomes by postinsertion method. In the latter case, anti-ICAM-1 antibody was fragmented, and the generated mFab' fragments were coupled to the distal ends of MPS encapsulated liposomes. Since stable colloidal properties of liposomes are critical for the application, size distribution and zeta potential of the prepared liposomes were characterized by dynamic light scattering studies.

\subsubsection{To Lyophilize and Develop Stable MPS Encapsulated Targeted Liposomes}

The purpose of this specific aim was to improve the shelf-life of the targeted liposomes. MPS-L ${ }^{\mathrm{cRGD}}$ and MPS- $\mathrm{L}^{\mathrm{Ab}}$ formulations were prepared in the presence of intra and extraliposomal lyoprotectants. The formulations were then subjected to lyophilization process to preserve the liposomes as dry powder. As a result of lyophilization, the original liposome properties were retained with minimal or no damage to the reconstituted liposomes. 


\subsubsection{To Modulate Pulmonary Edema via MPS-L ${ }^{\text {cRGD }}$ Targeting to $\alpha v \beta 6$ Integrins}

An IL-1 $\beta$ instilled rat ALI model was used to investigate the effect of MPS-L ${ }^{\text {cRGD }}$ on pulmonary edema. MPS- $\mathrm{L}^{\mathrm{cRGD}}$ were nebulized to bind to $\alpha \mathrm{v} \beta 6$ integrins of alveolar epithelium and inhibit pulmonary edema. Simultaneously, the effect of MPS on inhibition of pro-inflammatory mediators production was investigated. BALF samples were collected for analysis of protein, cytokines, chemokines and neutrophils.

\subsubsection{To Impede Neutrophil Migration via MPS-L ${ }^{\mathrm{Ab}}$ Targeting to ICAM-1 Receptors}

MPS-L ${ }^{\mathrm{Ab}}$ were targeted to ICAM-1 receptors of alveolar endothelium to attenuate neutrophil migration into alveoli. In this study, MPS-L ${ }^{\mathrm{Ab}}$ were administered by intravenous route prior to IL- $1 \beta$ instillation in rats. MPS- ${ }^{A b}$ disrupt the interaction of $\beta 2$ integrins of neutrophils with endothelial ICAM-1 receptors, and thereby attenuate neutrophil migration. BALF samples were collected for analysis of neutrophils and protein content. 


\section{CHAPTER 3. HPLC METHOD FOR DETERMINATION OF LIPIDS IN LIPOSOMES}

\subsection{Introduction}

Liposomes are lipid vesicles prepared by dispersing lipids in an aqueous phase. Liposomes were used as a carrier of drugs. ${ }^{252}$ One of the most significant advantages of using liposomes as a drug delivery carrier is the reduced toxicity and improved therapeutic efficacy. While different lipids could be used for formulating drugs in liposomes; invariably most formulations contain phospholipids and CHOL. Most commonly used phospholipids are egg phosphatidylcholine, hydrogenated soy phosphatidylcholine, 1, 2-distearoyl-sn-glycero-3-phosphocholine and 1, 2-dipalmitoylsn-glycero-3-phosphocholine. Several liposome formulations contain different anticancer agents, e.g., paclitaxel, doxorubicin and amphotericin. ${ }^{253,254}$ These formulations contain HSPC, CHOL and $\alpha$-tocopherol in product-specific ratios. There are several reported high performance liquid chromatography (HPLC) methods in the literature that use silica columns with UV detection, wide and varied mobile phase compositions of organic solvents (acetonitrile, hexane, methanol and isopropanol) and water for detection and estimation of phospholipids. ${ }^{255,256}$ Separation of several classes of phospholipids was achieved using silica column by gradient elution with hexane-isopropanol-water mobile phase. ${ }^{257,258}$ While estimation of CHOL and other phospholipids in liposome formulation has been reported, ${ }^{259,260}$ some methods require lipid extraction from samples prior to injecting into HPLC system, ${ }^{261}$ whereas others use complicated gradient elution for separation. ${ }^{259}$ Recently, evaporative light scattering detection (ELSD) has been used for estimation of CHOL and other phospholipids in liposome based formulations. ${ }^{262}$ While the methods that use ELSD are considered universal, they are cumbersome to reproduce, calibration of standards require non-linear regression analysis due to varying sensitivity of the detector and often give results with high variations compared to UV detection methods. In this report, we describe a rapid HPLC method for separation and simultaneous estimation of HSPC and CHOL components of liposome formulations. This method uses a C8 column, isocratic mobile phase and UV detection at $205 \mathrm{~nm}$. The results generated show that this method can also be used to quantitate $\alpha$-tocopherol, an anti-oxidant used in liposome formulations.

\subsection{Experimental Section}

\subsubsection{Materials}

CHOL used for calibration standard was purchased from National Institute of Standards and Technology (NIST, Gaithersburg, MD). HSPC and CHOL were purchased from Avanti Polar Lipids (Alabaster, AL) and were of research grade. Anti-oxidant, $\alpha-$ tocopherol was purchased from Sigma (St.Louis, MO). HPLC grade methanol, water, 
monobasic ammonium phosphate and $85 \% \mathrm{w} / \mathrm{v}$ phosphoric acid $\left(\mathrm{H}_{3} \mathrm{PO}_{4}\right)$ were purchased from Fisher Scientific (Suwannee, GA).

\subsubsection{Methods}

\subsubsection{High Performance Liquid Chromatography}

The HPLC system was equipped with Waters Alliance System (Waters ${ }^{\circledR}$, Milford) consisting of vacuum degasser, temperature controlled well-plate auto-sampler, column thermostat, quaternary pump and photo diode array detector. Chromatographic analysis was performed using a Hypersil BDS C8 $\left(15 \mathrm{~cm} \times 4.6 \mathrm{~mm}\right.$ i.d., $5 \mu \mathrm{m}$ particle size, $80^{\circ} \mathrm{A}$ pore size) column (Alltech, Deerfield, IL) at column temperature, $60^{\circ} \mathrm{C}$. The analytical column was protected with a disposable pre-column (Alltech, Deerfield, IL). Mobile phase flow rate was set to $1.0 \mathrm{~mL} / \mathrm{min}$ with a run time, $30 \mathrm{~min}$. The diode array detector was operated at $205 \mathrm{~nm}$ with $4 \mathrm{~nm}$ of bandwidth and slit setting, off reference mode and 2 $\mathrm{s}$ response time setting. Injection volume was set at $50 \mu \mathrm{L}$. An isocratic mobile phase containing $50 \mathrm{mM}$ solution of ammonium phosphate buffer (pH 3.0)-methanol (15:85\% v/v) was used.

\subsubsection{Preparation of Reagents}

Ammonium phosphate buffer $(50 \mathrm{mM}, \mathrm{pH} 3.0)$ was prepared by dissolving ammonium phosphate in water and adjusting the $\mathrm{pH}$ to 3.0 with $85 \% \mathrm{w} / \mathrm{v} \mathrm{H}_{3} \mathrm{PO}_{4}$. The clear solution was filtered through a $0.45 \mu \mathrm{m}$ nylon membrane filter and stored at room temperature. Mobile phase was prepared by mixing the buffer solution and methanol in 15:85\% v/v. Standard stock solutions of CHOL $(1.0 \mathrm{mg} / \mathrm{mL})$ and HSPC $(2.0 \mathrm{mg} / \mathrm{mL})$ were prepared using methanol. Intermediate stock solutions were prepared by mixing the stock solutions of CHOL and HSPC in the product-specific ratio to bracket the target concentration for analysis. Calibration standards were prepared by further diluting the intermediate solution. Five levels of calibration standards were prepared by diluting the intermediate solution. Mobile phase was used as diluent for intermediate and calibration standard solutions. The calibration standards used for liposome based MPS analysis were prepared in the range of 20-200 and 40-400 $\mu \mathrm{g} / \mathrm{mL}$ for CHOL and HSPC, respectively. For other drug formulations, similar preparations were made based on the lipid ratio in the particular formulation.

\subsubsection{Preparation of Liposome Formulations for HPLC Analysis}

Liposome based formulations were prepared as described elsewhere. The samples for HPLC injections were prepared by diluting the liposome formulations with mobile phase. To bring the concentration of the samples into the calibration range, the required dilution depends on the expected concentration of CHOL and HSPC in samples. 


\subsection{Results and Discussion}

The HPLC method described here was developed for estimation of CHOL and HSPC (Figure 3.1) following the FDA and international conference on harmonization of technical requirements for registration of pharmaceuticals for human use (ICH) guidelines. ${ }^{263,264}$ Linearity, accuracy, precision, specificity, quantitation limit and robustness of the method were tested to ensure that the method is suitable for identification and quantitation of CHOL and HSPC components of liposome formulations. Chromatographic performance data for a typical run are presented in Table 3.1. Resolution of 2.0 or greater is desired for critical band pair. Critical resolution of 2.543 was observed between CHOL and HSPC peaks. Tailing factors for both CHOL and HSPC are $<1.20$. Retention factor in the range of $0.5<\mathrm{k}^{\prime}<20.0$ is desired to separate the first peak from void time and to avoid higher retention time for the last band. Retention time of 4.316 and 6.205 were found for CHOL and HSPC, respectively. Retention factors of the CHOL and HSPC peaks were optimized by varying the mobile phase composition. However, it was necessary to increase column temperature to $60^{\circ} \mathrm{C}$ to achieve acceptable resolution between critical band pair of CHOL and HSPC.

\subsubsection{Linearity and Range}

Linearity of the calibration standards was tested in the concentration rage, 20.0-200.0 and $40.0-400 \mu \mathrm{g} / \mathrm{mL}$ for CHOL and HSPC, respectively. Five levels of calibration standards were prepared at various concentration levels. Correlation coefficients (r) were found to be $>0.999$ for both CHOL and HSPC (Table 3.2). Typical chromatogram of a standard solution is shown in Figure 3.2.

\subsubsection{Accuracy}

The accuracy study was performed to determine the closeness between the true concentration value and the experimental results. CHOL and HSPC were spiked into the excipients that include other lipids, anti-oxidant and MPS, expected to be present in the formulations. Spike recovery was performed at three levels (500, 100 and 150\%) of the target concentration. For each level, three preparations were performed. Table 3.3 summarizes the results from accuracy experiments. Average recovery of 99.80 and $100.14 \%$ were observed for CHOL and HSPC, respectively, which is within $\pm 2.0 \%$ of normally accepted value.

\subsubsection{Precision}

Instrument precision was performed as part of each sequence run at the beginning of the sequence. Six injections of the target level of calibration standard were performed and the data were evaluated. Table 3.4 shows the mean retention and area response for both $\mathrm{CHOL}$ and HSPC. Relative standard deviations (RSDs) for both CHOL and HSPC are 


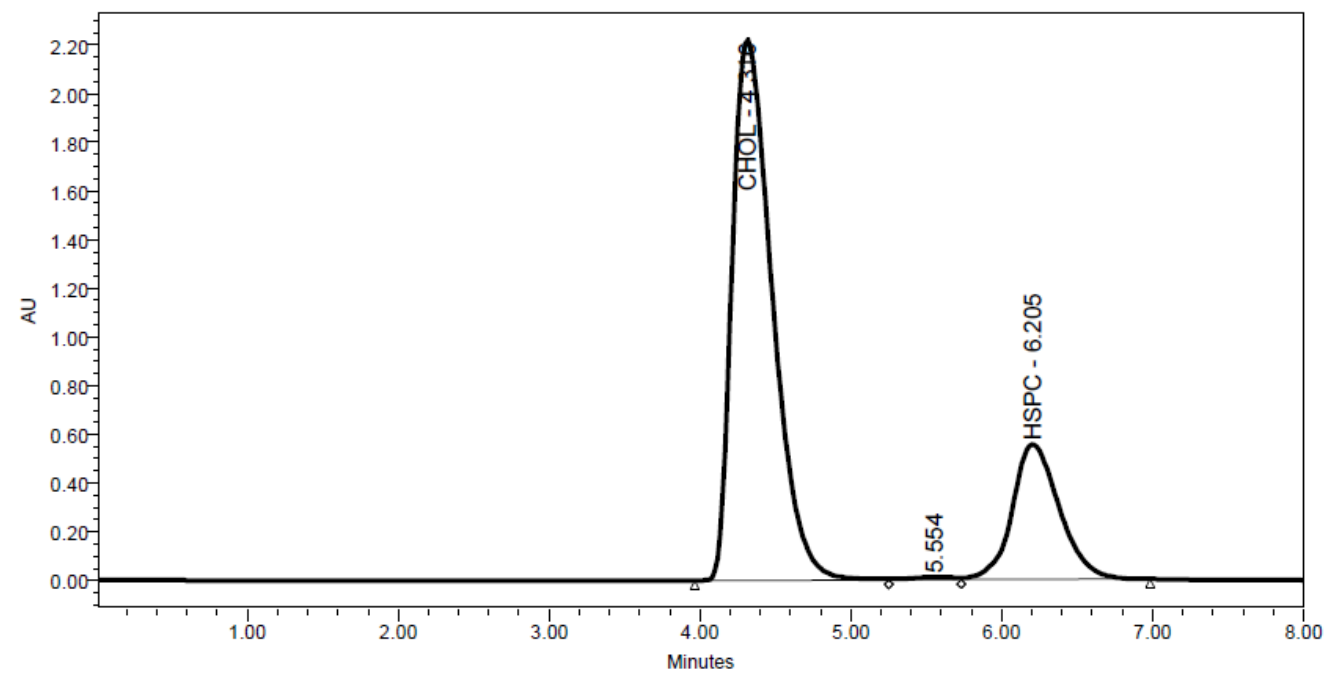

Figure 3.1 Chromatogram of calibration standards of CHOL and HSPC.

Table 3.1 Chromatographic performance data of the method.

\begin{tabular}{lccccc}
\hline Analyte & Retention time (min) & Tailing factor $^{\mathrm{a}}$ & Retention factor $^{\mathrm{b}}$ & Plate count $^{\mathrm{c}}$ & $\begin{array}{c}\text { Resolution between } \\
\text { critical band pair }\end{array}$ \\
\hline CHOL & 4.316 & 1.025 & 7.362 & 5440 & 2.543 \\
HSPC & 6.205 & 1.206 & 11.41 & 6826 & - \\
\hline
\end{tabular}

${ }^{\mathrm{a}}$ Tailing is defined as $\mathrm{W}_{0.05} / 2 \mathrm{t}_{\mathrm{w}}$, where $\mathrm{W}_{0.05}$ is peak width at $5 \%$ of peak height (min), $\mathrm{t}_{\mathrm{w}}$ is distance between peak front and peak retention measured at $5 \%$ of the peak height (min). ${ }^{b}$ Retention factor is defined as $\left(t_{R}-t_{0}\right) / t_{0}$, where $t_{R}$ is retention time of peak (min) and $t_{0}$ is void time (min). Void time $=0.5$ min for the method. ${ }^{c}$ Plate count is defined as $16\left(t_{R} / W_{B}\right)^{2}$, where $W_{B}$ is the tangent peak width (min). 
Table 3.2 Summary of linear regression data for calibration standards.

\begin{tabular}{lcccc}
\hline & \multicolumn{3}{c}{ Linear regression parameters } \\
\cline { 2 - 5 } Analyte & Intercept & Slope & Correlation coefficient $(\mathrm{r})$ & Coefficient of determination $\left(\mathrm{r}^{2}\right)$ \\
\hline CHOL & 6.641 & 11.239 & 0.99998 & 0.99995 \\
HSPC & 3.689 & 5.604 & 0.99999 & 0.99999 \\
\hline
\end{tabular}

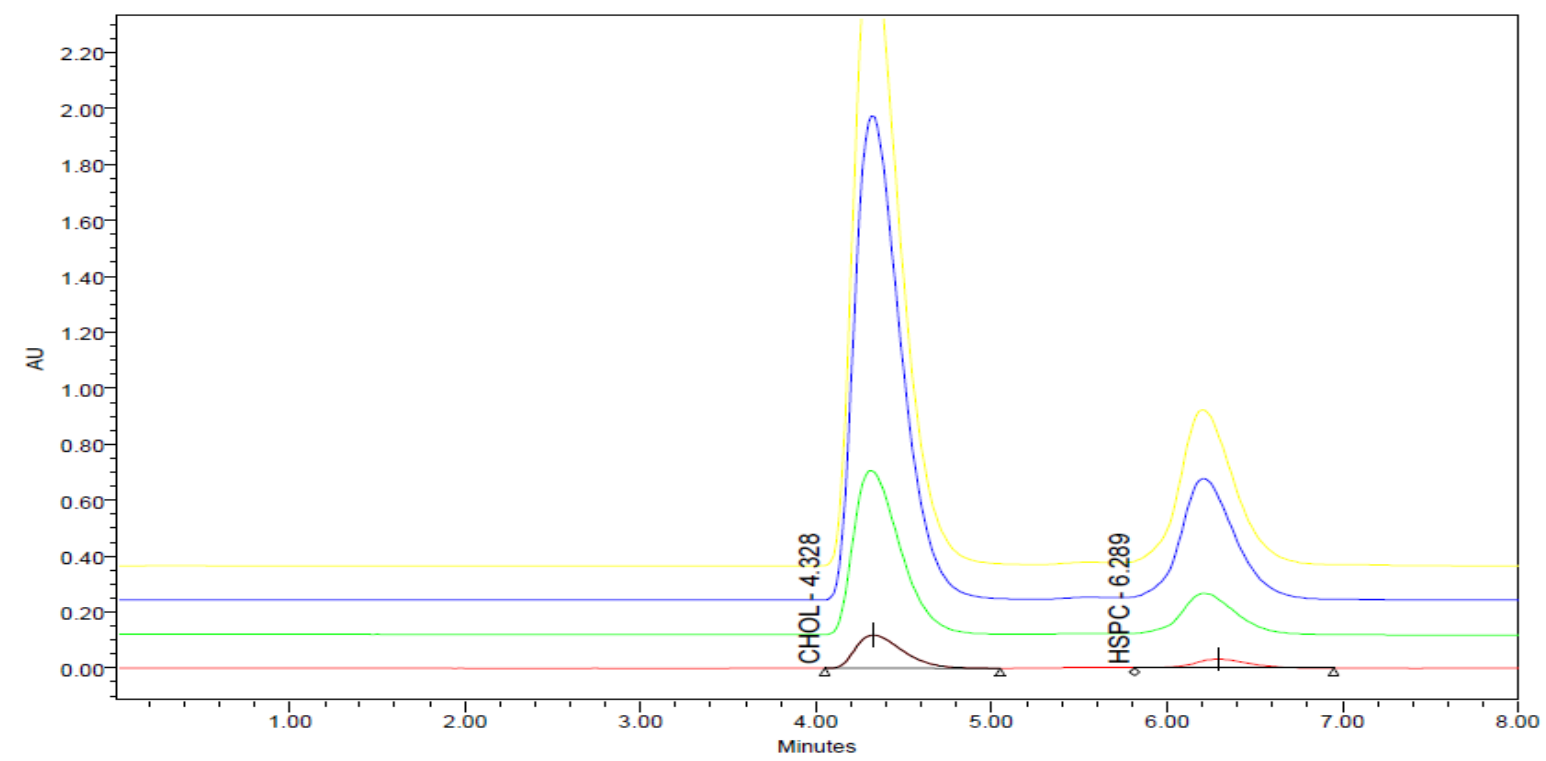

Figure 3.2 Typical chromatogram of linearity of standards containing CHOL and HSPC. 
Table 3.3 Summary of method accuracy results.

\begin{tabular}{cccccc}
\hline Analyte & $\begin{array}{c}\text { Recovery solution at } \\
\text { target level }(\%)\end{array}$ & $\begin{array}{c}\text { Theoretical } \\
\text { concentration }(\mu \mathrm{g} / \mathrm{mL})\end{array}$ & $\begin{array}{c}\text { Recovered average } \\
\text { concentration }(\mu \mathrm{g} / \mathrm{mL})\end{array}$ & $\begin{array}{c}\text { RSD }(\%) \\
\text { Analytical } \\
\text { recovery }^{\mathrm{a}}(\%)\end{array}$ \\
\hline \multirow{3}{*}{ CHOL } & 50 & 81.95 & $81.73(0.449)$ & 0.268 & 99.73 \\
& 100 & 163.900 & $163.71(0.507)$ & 0.115 & 99.88 \\
HSPC & 150 & 245.850 & $245.651(0.598)$ & 0.080 & 99.91 \\
& 50 & 112.682 & $112.931(0.416)$ & 0.220 & 100.22 \\
& 100 & 225.363 & $225.509(0.683)$ & 0.100 & 100.06 \\
\end{tabular}

${ }^{a}$ Results are based on six data points. Standard deviation is given in parenthesis.

Table 3.4 Summary of method precision results.

\begin{tabular}{lccc}
\hline & Analyte & Average concentration ${ }^{\mathrm{a}}(\mathrm{mg} / \mathrm{mL})$ & RSD (\%) \\
\hline Day 1 & CHOL & $22.433(0.276)$ & 1.16 \\
& HSPC & $31.692(0.730)$ & 0.216 \\
Day 2 & CHOL & $21.887(0.050)$ & 0.096 \\
\multirow{2}{*}{ Between days 1 and 2 } & HSPC & $31.888(0.307)$ & 1.075 \\
& HOL & $22.705(0.236)$ & 0.919 \\
& HSPC & $31.609(0.367)$ & 1.245 \\
\hline
\end{tabular}

${ }^{\mathrm{a}}$ Based on 12 data points. Standard deviation is given in parenthesis. 
$<2.0 \%$, which indicates sufficient instrument reproducibility of the method. For sample precision measurements, six replicate samples were prepared and analyzed on the first day. For each sample preparation, three injections were performed. On the second day, the same analyst prepared a second set of six samples from the same sample vial and independently analyzed on the same system. The RSD values are found to be $<2.0 \%$ for both CHOL and HSPC on each day and $<2.0 \%$ between days 1 and 2 . These results clearly indicate sufficient sample repeatability with this method.

\subsubsection{Quantitation Limit}

There are at least four different ways to determine quantitation limits of analyte, and signal-to-noise ratio $(\mathrm{S} / \mathrm{N})$ is one of the most commonly used procedures. We used this procedure for determining quantitation limits of CHOL and HSPC. Stock solutions of individual lipid components, CHOL and HSPC were progressively diluted and signal-tonoise ratios for $\mathrm{CHOL}$ and $\mathrm{HSPC}$ were determined until a minimum $\mathrm{S} / \mathrm{N}$ ratio of 10 was achieved. Quantitation limits of 0.5 and $10.0 \mu \mathrm{g} / \mathrm{mL}$ were observed for CHOL and HSPC, respectively. Previously, a detection limit of $1.5 \mu \mathrm{g} / \mathrm{mL}$ was reported for CHOL in liposome formulations. ${ }^{256}$

\subsubsection{Robustness}

Robustness is the capacity of a method to remain unaffected by small, deliberate variations in method parameters and measures reliability of the method. To test the robustness of the method, we deliberately varied four parameters, $\mathrm{pH}$ of the buffer, column temperature, mobile phase composition and flow rate. The buffer solutions with $\mathrm{pH}$ values of 2.8, 3.0 and 3.2 were used to study the effect of $\mathrm{pH}$ variation on the performance of the method. With each variation of buffer $\mathrm{pH}$, analysis of a sample solution was performed and the chromatographic results were evaluated. Data were collected at three column temperature settings, 58,60 and $62^{\circ} \mathrm{C}$ to study the effect of column temperature variation on the performance of the method. With each variation of column temperature, analysis of a sample solution was performed and the chromatographic results were evaluated. The mobile phase flow rates were set at 1.3, 1.5 and $1.7 \mathrm{~mL} / \mathrm{min}$ to study the effect of mobile phase flow rate variation on the performance of the method. Three different mobile phase compositions $(13: 87,15: 85$ and $17: 83(\mathrm{v} / \mathrm{v})$ of buffer-methanol) were used to study the effect of varying the mobile phase composition on the performance of the method. Robustness data from the above experiments are summarized in Table 3.5. The retention times for both CHOL and HSPC decreased with increasing column temperature, mobile phase flow rate and $\mathrm{pH}$ of the buffer, which is expected. The chromatographic performance of the method does not change significantly. The retention times for CHOL and HSPC increased with increasing polarity of the mobile phase composition. However, it is important to note that the overall performance of the method remained unaffected. 
Table 3.5 Summary of robustness of the method.

\begin{tabular}{|c|c|c|c|c|c|c|c|c|c|c|}
\hline \multicolumn{2}{|l|}{ Parameters } & \multirow[t]{2}{*}{$\begin{array}{c}\text { Critical } \\
\text { resolution }\end{array}$} & \multicolumn{2}{|c|}{ Tailing factor } & \multicolumn{2}{|c|}{ Plate count } & \multicolumn{2}{|c|}{ Overall RSD ${ }^{\mathrm{a}}(\%)$} & \multicolumn{2}{|c|}{$\begin{array}{c}\text { Correlation } \\
\text { coefficient (r) }\end{array}$} \\
\hline \multirow{4}{*}{$\begin{array}{l}\text { Column } \\
\text { temperature }\left({ }^{\circ} \mathrm{C}\right)\end{array}$} & & & CHOL & HSPC & CHOL & HSPC & CHOL & HSPC & $\mathrm{CHOL}$ & HSPC \\
\hline & 58 & 3.68 & 1.02 & & 6026 & 7136 & 1.24 & 1.36 & 0.99992 & 0.99978 \\
\hline & 60 & 3.60 & 1.02 & 1.24 & 5884 & 6927 & 0.98 & 0.41 & 0.99988 & 0.99989 \\
\hline & 62 & 3.40 & 1.04 & 1.23 & 5436 & 6638 & 1.69 & 0.38 & 0.99975 & 0.99971 \\
\hline \multirow{3}{*}{$\begin{array}{l}\text { Flow rate } \\
(\mathrm{mL} / \mathrm{min})\end{array}$} & 1.3 & 3.66 & 1.02 & 1.23 & 5997 & 7206 & 1.85 & 1.47 & 0.99984 & 0.99963 \\
\hline & 1.5 & 3.60 & 1.02 & 1.21 & 5883 & 6927 & 0.87 & 0.94 & 0.99996 & 0.99981 \\
\hline & 1.7 & 3.38 & 1.03 & 1.21 & 5513 & 6505 & 0.65 & 1.02 & 0.99977 & 0.99978 \\
\hline \multirow[t]{3}{*}{ Mobile phase } & $13: 87$ & 3.45 & 1.03 & 1.21 & 5201 & 6498 & 1.74 & 1.15 & 0.99983 & 0.99984 \\
\hline & $15: 85$ & 3.60 & 1.02 & 1.21 & 5884 & 6927 & 0.89 & 1.62 & 0.99993 & 0.99993 \\
\hline & $17: 83$ & 4.25 & 1.02 & 1.22 & 4986 & 6334 & 1.60 & 0.71 & 0.99971 & 0.99987 \\
\hline \multirow[t]{3}{*}{ Buffer $\mathrm{pH}$} & 2.8 & 3.38 & 1.04 & 1.22 & 5115 & 6728 & 1.29 & 0.68 & 0.99987 & 0.99975 \\
\hline & 3.0 & 3.60 & 1.02 & 1.22 & 5884 & 6928 & 0.71 & 0.95 & 0.99992 & 0.99989 \\
\hline & 3.2 & 3.36 & 1.04 & 1.22 & 4970 & 6725 & 0.80 & 1.09 & 0.99986 & 0.99977 \\
\hline
\end{tabular}

${ }^{a}$ For each sequence run, a calibration standard solution was injected at the beginning of the sequence, after the five levels of calibration standards (two injections) and at the end of the sequence (two injections) to verify the system suitability of the overall run sequence. The $\%$ RSD data are based on the response (area) of these 10 system suitability injections for each run. 


\subsubsection{Conclusions}

A rapid isocratic HPLC method for simultaneous quantitation of CHOL and HSPC in liposome formulations was developed. The method is linear, precise, accurate, sensitive, robust and specific for quantitation of CHOL and HSPC in liposome formulations. In addition, this method is applicable to several of the liposome formulations for quantitation of CHOL and HSPC, and does not require preprocessing of the samples. This method also improved the quantitation limits of CHOL compared to other reported methods. 


\section{CHAPTER 4. MPS ENCAPSULATION AND SURFACE MODIFICATION OF LIPOSOMES}

\subsection{Introduction}

Methylprednisolone sodium succinate treatment is widely used in the treatment of inflammatory diseases. ${ }^{85,265}$ MPS is more effective anti-inflammatory agent than are nonsteroidal anti-inflammatory drugs. However, the systemic administration of MPS is often accompanied by substantial side effects, especially when long-term treatment is used. Due to the fast clearance of MPS from the body, frequent high-dose intravenous (i.v.) and/or oral administration is required to achieve efficacy, but this affects many tissues and causes serious side effects. Therefore, improvement of the therapeutic efficacy would be beneficial. A systemic "targeted" administration will bring the MPS selectively to the inflamed areas and increases the therapeutic index of MPS. This can be achieved by MPS delivery via liposomes. Although, MPS delivery via liposomes is one of the earliest attempts to use liposomes as drug carriers, until now no liposomal MPS formulation has reached clinical application. A systemic liposome formulation needs to fulfill several key requirements in order to be efficacious enough to justify development as a drug: I) liposome formulation should accumulate at the inflammation site as a result of prolonged circulation due to reduction in uptake by the RES, II) low drug leakage rate during the circulation, III) liposomal formulation should be chemically and physically stable during storage, IV) liposomes should have a high [drug/lipid] ratio to avoid toxicity induced by high liposome dose and V) high drug encapsulation is required. Herein, I describe the development of MPS liposome formulations using passive and active loading strategies. In both the strategies, the influence of formulation composition and process variables were investigated comprehensively to improve the MPS encapsulation efficiency and meet the above criteria. However, the formulation based on a novel acting loading approach, calcium acetate transmembrane gradient efficiently loaded MPS into liposomes. Approximately, 63\% w/v MPS encapsulation efficiency was achieved using the acetate gradient method. In addition, MPS-L were surface modified with cRGDpeptide by post-insertion method to exert control on the incorporation of targeting ligand. In this method, an incorporation efficiency of $45.3 \%$ (molar) was achieved.

In this chapter, the influence of formulation composition, process parameters and loading strategies were investigated to obtain an optimized MPS encapsulated formulation for use in in vivo studies.

\subsection{Materials}

HSPC, 1, 2-dipalmitoyl-sn-glycerophosphoethanolamine-poly (ethylene glycol) $2000^{-}$ amine (DSPE-PEG 2000 ), 1, 2-dipalmitoyl-sn-glycerophosphoethanolamine-poly (ethylene

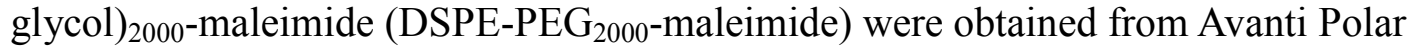
Lipids (Birmingham, AL). MPS and CHOL were purchased from Sigma (St.Louis, MO). cRGD-peptide was purchased from Peptide International Inc. (Louisville, KY). PBS and 
HEPES buffers were purchased from cellgro Inc. (Herndon, VA). All other chemicals were obtained from Sigma or Fisher Scientific.

\subsection{Methods}

\subsubsection{Preparation of MPS-L by Passive Loading (Bangham Method)}

Liposomes were prepared with a composition of HSPC: CHOL: DSPE-PEG 2000 in 50: 45: 3: 2 molar ratios. The lipids were solubilized in chloroform-methanol solution and subjected to evaporation to form a thin lipid film. Hydration of the lipid film was carried with HEPES buffer (50 mM, pH 6.5) containing MPS at $100 \mathrm{mg} / \mathrm{mL}$. The resulting MLVs were extruded through 100/200 nm sandwiched polycarbonate membrane filters using liposome extruder (LIPEX ${ }^{\mathrm{TM}}$, Northern Lipids, Canada). MLVs were subjected to the required number of extrusion passes to form small unilamellar vesicles (SUVs) in the size range, $100-120 \mathrm{~nm}$. This was followed by the incubation of SUVs with cRGDpeptide solution, $\mathrm{pH} 6.5$, overnight at $25^{\circ} \mathrm{C}$. The conjugation reaction was quenched with $100 \mu \mathrm{L}$ of $1 \mu \mathrm{M}$ of 1-ethyl-3-(3-dimethylaminopropyl)-carbodiimide (EDC; Sigma, MO). Unconjugated free RGD-peptide and free MPS were isolated by dialysis at $4^{\circ} \mathrm{C}$ for $6 \mathrm{~h}$ using equilibrium chambers (Harvard Apparatus, MA), separated by a 10,000 Da MWCO membrane. The dialysate was subjected to HPLC analyses to determine MPS encapsulation and cRGD-conjugation efficiency. The surface modified liposomes were stored at $4^{\circ} \mathrm{C}$ until further use.

\subsubsection{Active Loading of Amphipathic MPS into Liposomes}

Remote loading was performed using calcium acetate transmembrane gradient method. Ca.acetate $]_{\text {liposome }}>>[\mathrm{Ca} \text {.acetate }]_{\text {medium }}$ transmembrane gradient was used as the driving force for the remote loading of MPS, an amphipathic weak acid. Such a gradient is characterized by an excess of the membrane-impermeable $\mathrm{Ca}^{+2}$ ions over the membranepermeable acetic acid; the leakage of acetic acid of the liposomes leads to creation of a $\mathrm{pH}$ gradient in which the $\mathrm{pH}_{\text {liposome }}>>\mathrm{pH}_{\text {medium. In such liposomes, the amphipathic weak }}$ acid, such as MPS in its unionized form is diffused into the slightly alkaline intraliposomal aqueous phase. Inside the aqueous core, it is de-protonated and becomes negatively charged. Now, the ionized MPS forms a poorly soluble calcium-MPS complex, which precipitates in the intraliposomal aqueous phase. The loading process continues till the exhaustion of intraliposomal acetate ion concentration. Lipids, $\mathrm{HSPC} / \mathrm{CHOL} / \mathrm{DSPE}-\mathrm{PEG}_{2000}$ were dissolved in ethanol and then were hydrated with 200 $\mathrm{mM}$ calcium acetate at $65^{\circ} \mathrm{C}$. The large MLVs formed upon lipid hydration were extruded at $65^{\circ} \mathrm{C}$ through sandwiched polycarbonate filters $(100 / 200 \mathrm{~nm})$. Approximately, $100-120$ $\mathrm{nm}$ sized liposomes were obtained. To create the transmembrane calcium acetate gradient, the calcium acetate of the external liposome medium was replaced by $0.9 \%$ $\mathrm{NaCl}(\mathrm{w} / \mathrm{v})$ at $4^{\circ} \mathrm{C}$ by dialysis. MPS was dissolved in $0.9 \% \mathrm{NaCl}$ to a concentration of 19 $\mathrm{mM}(9 \mathrm{mg} / \mathrm{mL})$ and mixed with preformed liposome dispersion (30 $\mathrm{mM}$ phospholipids) 
in which a transmembrane calcium acetate gradient was created. The remote loading was achieved by incubation of the liposome dispersion at $48^{\circ} \mathrm{C}$ (near the Tm of HSPC, i.e. $52^{\circ} \mathrm{C}$ ) for $6 \mathrm{~min}$, and then liposomes were cooled to $4^{\circ} \mathrm{C}$ and dialyzed against $0.9 \% \mathrm{NaCl}$ to remove any residual traces of acetate ion. After dialysis, MPS and phospholipid concentrations were $12.03 \mathrm{mM}$ and $27.2 \mathrm{mM}$, respectively.

\subsubsection{Synthesis of DSPE-PEG 2000 -maleimide-cRGD Conjugate}

DSPE-PEG 2000 -maleimide-cRGD conjugate was synthesized and incorporated into MPS encapsulated liposomal bilayers for targeting to lungs via the integrin receptors. The conjugate was synthesized as described in Figure 4.1. Briefly, 11.34 mg of DSPE$\mathrm{PEG}_{2000}$-maleimide and $1.16 \mathrm{mg}$ of cRGD peptide were dissolved in $100 \mu \mathrm{L}$ of HEPES buffer (50 mM, pH 6.5) containing TCEP and $1 \mathrm{mM}$ disodium EDTA. The reaction was carried overnight at $25^{\circ} \mathrm{C}$ on a tube shaker (Barnstead/Thermolyne, IA). The product was dialyzed in 1000 Da MWCO Slide-A-Lyzer ${ }^{\circledR}$ dialysis cassettes (Pierce, Rockford, IL) against HEPES buffer ( $\mathrm{pH} 6.5)$ at $4^{\circ} \mathrm{C}$ for $6 \mathrm{~h}$. Followed by dialysis, the product was subjected to further de-salting on micro bio-spin chromatography columns (Bio-Rad, Hercules, CA). The product was then lyophilized and stored at $-20^{\circ} \mathrm{C}$ until further use. The product was characterized by vMALDI. For vMALDI analysis, the product $(\sim 1 \mathrm{nM})$ was purified with a ZipTip C18 spin column using the procedure recommended by the manufacturer (Millipore, Billerica, MA). The purified product solution was eluted with 2 $\mu \mathrm{L}$ of $\alpha$-cyano-4-hydroxycinnamic acid ( $\alpha$-CHCA) matrix solution $(2.5 \mathrm{mg} / \mathrm{mL}$ in ACN/Water/TFA mixture) on a micro-column. The product/matrix mixture was spotted on a vMALDI plate and air-dried at room temperature. The product was analyzed with vMALDI-LTQ mass spectrometer (ThermoFinnigan, San Jose, CA) operated in positiveion mode. For the vMALDI source, the crystal-positioning system and auto spectrum filter (ASF) were enabled and ASF threshold was set at 500 counts for an MS scan. Automatic gain control (AGC) was enabled to adjust the number of laser shots and maintain the quality of vMALDI spectra. For an MS scan, high-mass range (m/z 500 4000) and normal scan rate were used. Product spectra were acquired and analyzed using Xcalibur software (ThermoFinnigan, San Jose, CA). The amount of residual free cRGD peptide was determined by Ellman reaction, ${ }^{266}$ which quantifies the sulfhydryl groups of cRGD peptide.

\subsubsection{Insertion of DSPE-PEG 2000 -maleimide-cRGD Conjugate into Liposomes}

DSPE-PEG 2000 -maleimide-cRGD conjugate was micellized and inserted into liposomes to exert control on the number of targeting ligands per liposome. A micellar solution of the conjugate was prepared by a procedure described elsewhere. ${ }^{267,268}$ Briefly, $28.9 \mathrm{mg}$ of the lyophilized conjugate was solubilized in $1000 \mu \mathrm{L}$ of water to form micellar solution. The resulting micellar solution was lysed in 5\% w/v Triton-X surfactant solution and the total cRGD peptide content was determined by Ellman reaction. Based on the liposome 


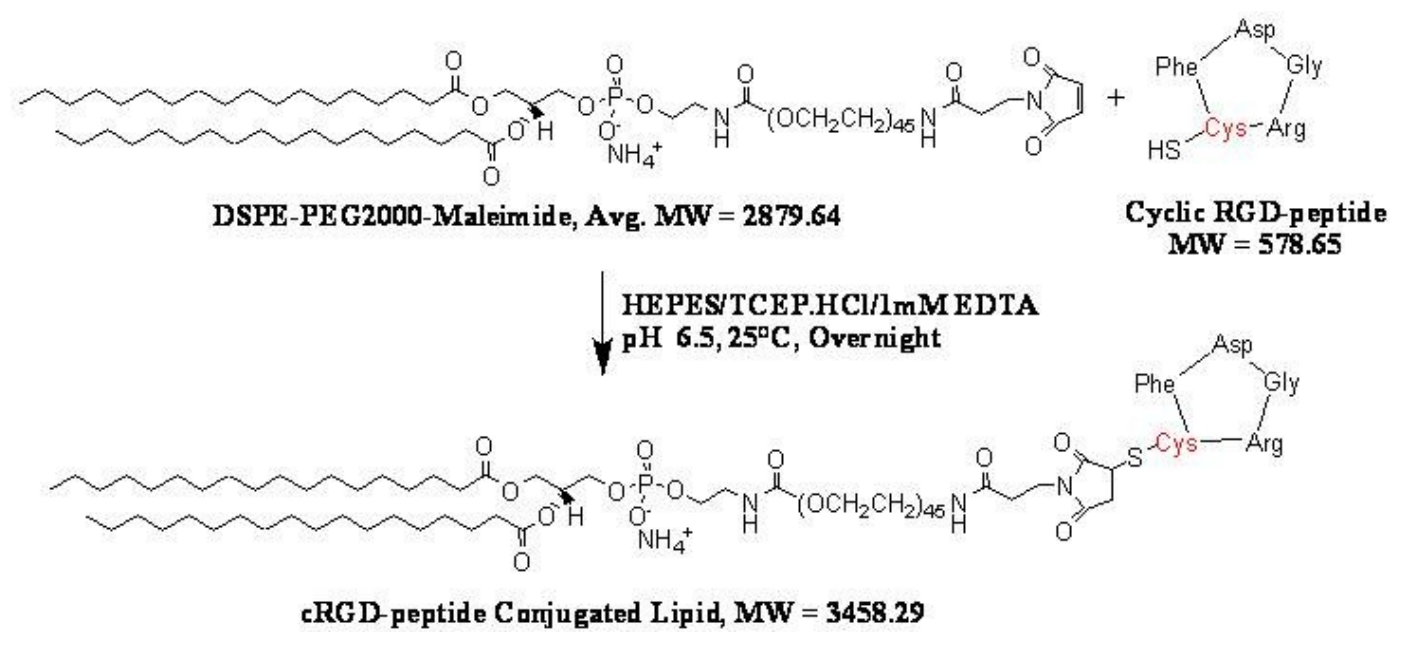

Figure 4.1 Schematic representation of Michael addition reaction between the lipid, DSPE-PEG 2000 -maleimide and cRGD-peptide.

The reaction was carried between equimolar concentrations of the lipid and the peptide. The synthesized conjugate was de-salted, and futher purified using dialysis cassettes (MWCO, $1000 \mathrm{Da}$ ) at $4^{\circ} \mathrm{C}$ for $6 \mathrm{~h}$. The peptide and phospholipid contents were estimated by Ellman reaction and phosphorus assay to determine the conjugation efficiency. The lipo-peptide conjugate was qualitatively characterized by MALDI-MS in positive ion mode. 
number, a specific micellar concentration of the conjugate was added to liposome suspension to obtain the required number $(1,500)$ of targeting ligands per liposome. The number of liposomes was determined by $\mathrm{N}_{\mathrm{VOL}}$ method. ${ }^{238}$ As illustrated in Figure 4.2, both micellar conjugate and liposomes were incubated together at $48^{\circ} \mathrm{C}$ for $6 \mathrm{~min}$ and stored overnight at $4^{\circ} \mathrm{C}$. The liposome formulation was then centrifuged $(1,500 \mathrm{xg}, 15$ min) in mini bio-spin chromatography columns to remove any unincorporated conjugate. Followed by centrifugation, liposome formulation was lysed with $5 \% \mathrm{w} / \mathrm{v}$ Triton-X surfactant solution, and cRGD peptide content was determined to estimate the conjugate incorporation efficiency.

In active loading strategy, MPS encapsulation was evaluated with respect to formulation composition and process parameters, before surface modification with cRGD-peptide.

\subsubsection{Chemical Characterization of Liposome Dispersion}

The total and intraliposome concentrations of MPS were quantified using RP-HPLC method. The concentrations of HSPC and CHOL were determined using HPLC method as described in Chapter 3.

\subsubsection{Physical Characterization of Liposome Dispersion}

Mean diameter (Zavg) and zeta potential of liposomes were evaluated by dynamic light scattering (DLS) studies using zetasizer ${ }^{\circledR}$ (Malvern Inc., Westborough, MA) equipped with a $4.5 \mathrm{~mW}$ diode laser as a light source operating at $670 \mathrm{~nm}$. Particle scattered photons were detected at $173^{\circ}$. Medium refractive index, 1.335 ; medium viscosity, 1.0 $\mathrm{mPa}$; and a dielectric constant of 80.0 were used as reference parameters for experimental investigations. Samples were suitably diluted with $0.22 \mu \mathrm{m}$ filtered HPLC water to avoid multi-scattering phenomena for Zavg and zeta potential determination. A Smoluchowski constant of 1.5 was used to measure zeta potential values from electrophoretic mobility. The mean diameter and zeta potential of liposome formulations were expressed in nanometers $(\mathrm{nm})$ and millivolts $(\mathrm{mV})$, respectively. Morphology was determined by negative staining of the SUV specimens on carbon support films by the single droplet procedure. $2 \% \mathrm{w} / \mathrm{v}$ aqueous solution of uranyl acetate $(\mathrm{pH} 6.5)$ was employed to stain the specimens. Specimens were studied using transmission electron microscope, functioning at $200 \mathrm{kV}$ (JEOL-FX 2000). A magnification of 50,000x was employed to acquire the electron micrograph.

\subsubsection{MPS Encapsulation Efficiency}

MPS encapsulation efficiency studies were carried to evaluate the MPS loading as a function of composition and process parameters. In passive loading strategy, formulation composition parameters include drug/lipid ratios, CHOL fraction and PEGylated lipid fraction. Process parameters include primarily, the effect of extrusion passes and pressure 
Step I: Micellization of Conjugate

cRGD-peptide or anti-ICAM-1 Ab conjugate

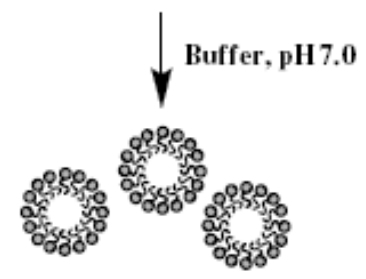

Conjugate Micelles

Step II: Post-insertion of Micelles into Pre-formed Liposomes

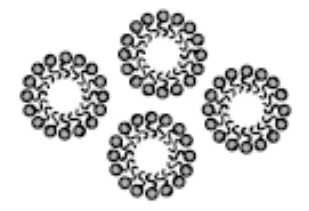

Conjugate Micelles

(1)
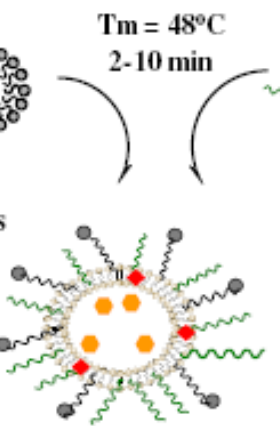

Targeted Liposomes

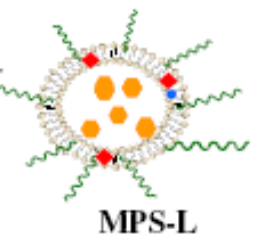

MPS-L rour - DSPE-PEG 200

mmo-Conjugate

Figure 4.2 Schematic representation of the insertion of the targeting conjugate by post-insertion technique.

The micelles of the conjugate were incubated with MPS encapsulated liposomes to yield targeted liposomes, MPS- $\mathrm{L}^{\mathrm{cRGD}}$. All targeting ligands on the external leaflet of the liposomes are presumed to be available for targeting. The conjugate insertion efficiency into liposomes was determined to be $34 \%$ (molar) as determined by Ellman reaction and phosphorus content analysis. 
on MPS encapsulation. Extrusion parameters do not have a significant influence on MPS encapsulation in active loading strategy as preformed small unilamellar liposomes were used for MPS incorporation. MPS encapsulation efficiency (\% EE) was calculated using the Equation 4.1

$$
\% \mathrm{EE}=\left(\mathrm{A}_{\mathrm{t}}-\mathrm{A}_{\mathrm{f}} / \mathrm{A}_{\mathrm{t}}\right) \quad \text { Equation } 4.1
$$

Where $A_{t}$ is the total amount of drug used for encapsulation and $A_{f}$ is the amount of free drug left in solution.

\subsubsection{In Vitro Leakage Kinetics of MPS from Liposomes}

The kinetics of MPS leakage in buffers was quantified using dialysis cassettes (MWCO, $10000 \mathrm{Da})$ equilibrated with HEPES buffer ( $\mathrm{pH} 7.4,50 \mathrm{mM})$. At definite time points, aliquots were taken and analyzed for MPS leakage.

\subsubsection{Mathematical Models}

Mathematical models were applied to describe the MPS release kinetics in liposome formulations. Higuchi and Korsenmeyer-Peppas (K-P) models were applied to describe the diffusion kinetics of MPS from liposomes, prepared by passive loading method. For active loading method (See Figure 1.6B), a mathematical model based on Rippie's assumptions was proposed to describe the drug release diffusion kinetics in liposomes (Figure 4.3). The same model was used to estimate the encapsulated drug $\left(A_{t}-A_{f}\right)$ in liposomes prepared by active loading method.

\subsection{Results and Discussion}

\subsubsection{Passive Loading Strategies}

\subsubsection{MPS Encapsulation Efficiency (\% EE): Influence of Drug/Lipid (D/L) Ratios}

Initial work was devoted for selection of phospholipid and characterizing its effect on MPS retention. Further work was extended to study the influence of D/L ratios on MPS encapsulation. HSPC/CHOL (55/45 mole\%; $27.2 \mathrm{mM}$ total lipids) liposomes were prepared with increasing amounts of MPS corresponding to D/L ratios from 0.1 to 0.8 . The liposomes were then dialyzed at $4^{\circ} \mathrm{C}$ for $6 \mathrm{~h}$ to remove unencapsulated drug, and the $\mathrm{D} / \mathrm{L}$ ratio was measured after quantitation of MPS inside the liposomes.As shown in Figure 4.4, D/L ratio as high as 0.6 (molar) was achieved. However, a stable encapsulation could not be achieved, and a substantial fraction $(\sim 92 \% \mathrm{w} / \mathrm{v})$ was desorbed into the external medium in $6 \mathrm{~h}$. 


$$
\mathrm{D}_{\mathrm{L}}{ }^{\mathrm{tot}}=[\mathrm{Du}]_{\mathrm{L}}+[\mathrm{Di}]_{\mathrm{L}}+\left[\mathrm{Ca}^{+2}-\mathrm{D}\right]_{\mathrm{L}}
$$

Where $\mathrm{D}_{\mathrm{L}}{ }^{\text {tot }},[\mathrm{Du}]_{\mathrm{L}},[\mathrm{Di}]_{\mathrm{L}}$ and $\mathrm{Ca}^{+2}-\mathrm{D}$ are total drug, unionized drug, ionized drug,and drug precipitate inside liposomes, respectively.

$$
[\mathrm{Di}]=[\mathrm{Du}] \cdot 10^{\mathrm{pH}-\mathrm{pKa}}
$$

Due to high $\mathrm{pH}$ inside the liposome, $\mathrm{K}^{\prime}<<\mathrm{K}$ leading to $[\mathrm{Du}]_{\mathrm{L} \ll}[\mathrm{Di}]_{\mathrm{L}}$ And due to high calcium gradient inside the liposomes, $[\mathrm{Di}]_{\mathrm{L},}\left[\mathrm{Ca}^{+2}-\mathrm{D}\right]_{\mathrm{L}}$ Therefore, at a given time, $\mathrm{D}_{\mathrm{L}}{ }^{\text {tot }}{ }\left[\mathrm{Ca}^{+2}-\mathrm{D}\right]_{\mathrm{L}}$.

Outside the liposomes, $\mathrm{D}_{0}{ }^{\text {tot }}$ is estimated by analysis of the dialysate according to the following equation $\mathrm{D}_{0}{ }^{\mathrm{tot}}=[\mathrm{Du}]_{\mathrm{O}}+[\mathrm{Di}]_{\mathrm{O}}$ which is written as $\mathrm{D}_{\mathrm{o}}{ }^{\text {tot }}=[\mathrm{Du}]_{\mathrm{O}}+[\mathrm{Du}]_{\mathrm{O}} \cdot 10^{\mathrm{pH}-\mathrm{pKa}}$

Where $\mathrm{D}_{0}{ }^{\text {tot }},[\mathrm{Du}]_{\mathrm{O}}$, and $[\mathrm{Di}]_{\mathrm{O}}$ are total drug, unionized and ionized drug concentrations outside the liposomes.

Figure 4.3 Schematic representation of the mathematical model based on Rippie's assumptions.

The model can be used to predict the drug diffusion in and out of particulate cores, where $\mathrm{pH}$ transmembrane gradient is maintained across the membranes. 


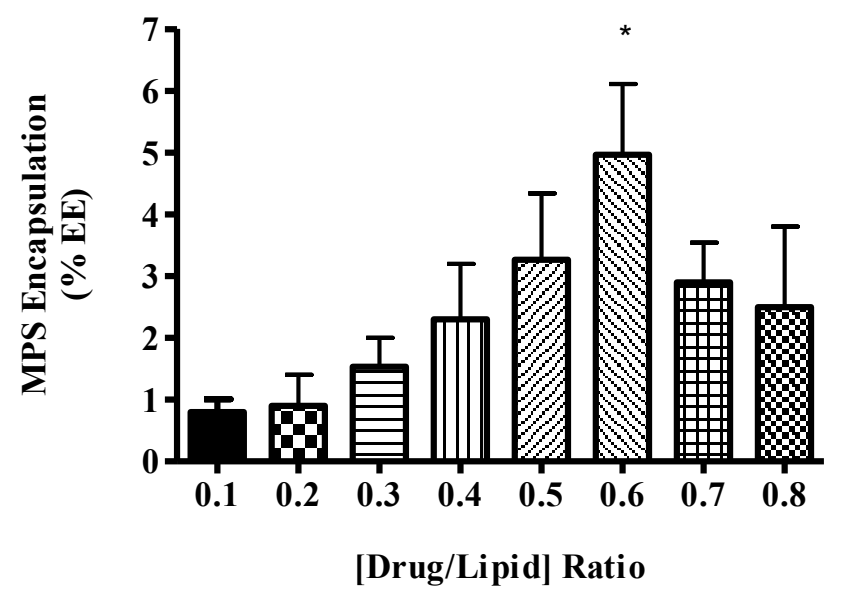

Figure 4.4 Graph depicting the MPS encapsulation efficiency as a function of drug/lipid ratios.

Each level is a mean \pm SEM of $\mathrm{n}=3$ samples. Total lipid content of MPS-L was $27.2 \mathrm{mM}$. $* p<0.05$ in comparison to $[\mathrm{D} / \mathrm{L}]$ at 0.1 or 0.2 . Statistical analysis was performed using Newman-Keuls test for multiple comparison (one-way ANOVA). 


\subsubsection{Influence of CHOL Incorporation on MPS encapsulation}

Basic composition of liposomes includes HSPC and CHOL. Being a saturated lipid, HSPC is stable to chemical degradation and avoids premature leakage of encapsulated drug from the liposomes in comparison to unsaturated phospholipids. CHOL was included in the formulation to enhance the rigidity of phospholipid bilayers. However, with CHOL, MPS entrapment efficiency was reduced, which may be due to phase separation of $\mathrm{CHOL}$ from the liposome formulation during hydration and extrusion processes. Phase separation and CHOL crystallization may induce pore formation in phospholipid bilayers and cause extensive drug leakage.As shown in Figure 4.5, formulation composition of $\mathrm{HSPC} / \mathrm{CHOL}$ at $55 / 45 \mathrm{~mole} \%$ showed a $\% \mathrm{EE}$ of 8.94 . Though, HSPC/CHOL at $65 / 35 \mathrm{~mole} \%$ showed the highest entrapment, a substantial amount of MPS leaked out in $6 \mathrm{~h}$ at $37^{\circ} \mathrm{C}$.

\subsubsection{Influence of PEG Incorporation on MPS Encapsulation}

PEG-lipids were incorporated into the liposome membrane to enhance the in vivo circulation half-life of liposomes. As shown in Figure 4.6, \% EE values containing 1-10 mole $\%$ of PEGylated lipid in the liposome formulations are in the range of $2-8 \% \mathrm{w} / \mathrm{v}$ of the MPS used for encapsulation. MPS encapsulation showed no significant effect till 5 mole\% of the incorporated PEGylated lipids. Usually, the PEGylated lipid concentration is limited to $5 \mathrm{~mole} \%$ in long circulating liposome formulations. In this range, MPS encapsulation efficiency is independent of PEGylated concentration.

\subsubsection{MPS Encapsulation: Influence of Extrusion Pressure}

Characteristics of the extrusion procedure (applied pressure, flow rate and pore size) as well as physical properties of the lipids and the lipid suspension (surface tension, bending modulus and concentration) may all play a role in the size and polydispersity of the vesicles produced. Understanding the role of these factors will contribute to the reproducibility achieved and should lead to production of more ideal vesicle solutions. Size and polydispersity of vesicles produced by extrusion depend on the number of times the solution is extruded, pore size of the extruded filters, ${ }^{269}$ lipid properties ${ }^{270}$ and

pressure. ${ }^{271}$ Optimization of extrusion pressure and cycles is critical for drug encapsulation. As shown in Figure 4.7, MPS encapsulation reduced with extrusion pressure.

\subsubsection{Drug Release Models}

The drug release data was analyzed with mathematical models, such as the Higuchi model and the Korsmeyer-Peppas equation. ${ }^{272}$ One of the most often used mathematical equations to describe the release rate of drugs from drug delivery systems is the Higuchi model, ${ }^{273}$ which is described by Equation 4.2: 


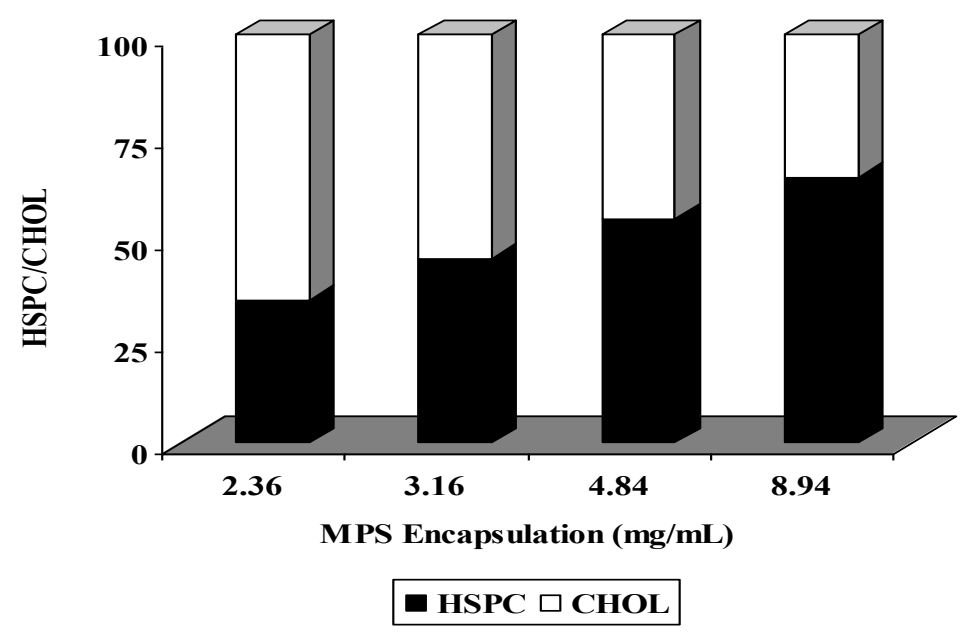

Figure 4.5 Graph depicting the MPS encapsulation efficiency as a function of CHOL fraction.

Each level is a mean \pm SEM of $n=3$ samples. Total lipid concentration was $27.2 \mathrm{mM}$.

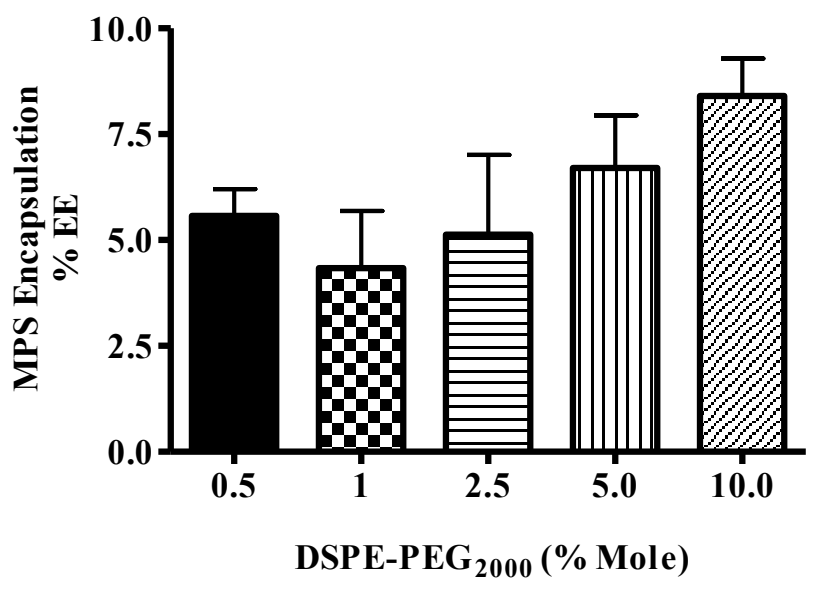

Figure 4.6 Graph depicting the MPS encapsulation efficiency as a function of PEGylated lipid incorporation.

Each level is a mean \pm SEM of $\mathrm{n}=3$ samples. From the graph, it is observed that there is no significant difference in MPS encapsulation as a function of PEGylated lipid concentration. 


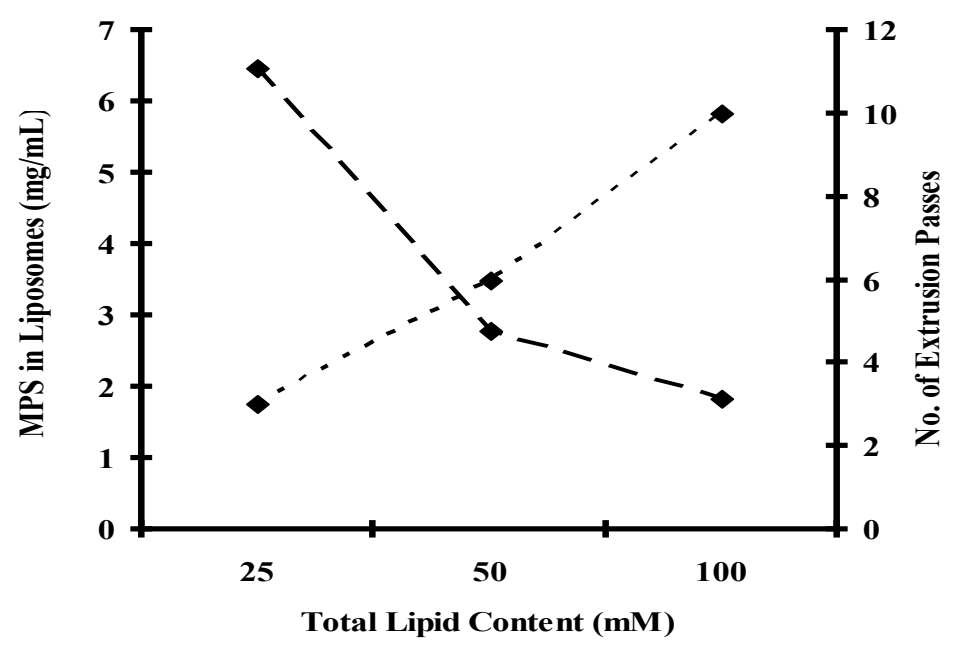

Figure 4.7 Graph depicting the MPS encapsulation efficiency as a function of number of extrusion cycles.

For all the levels of lipid content, an extrusion pressure of 250 psi was maintained. Each level is a mean \pm SEM of $n=3$ samples. 


$$
\mathrm{M}_{\mathrm{t}} / \mathrm{A}=\left[\mathrm{D}\left(2 \mathrm{C}_{0}-\mathrm{C}_{\mathrm{S}}\right) \mathrm{C}_{\mathrm{S}} \mathrm{t}\right]^{0.5} \text { for } \mathrm{C}_{0}>\mathrm{C}_{\mathrm{S}}
$$

\section{Equation 4.2}

The Higuchi equation was originally applied to dissolution and growth from solid particles. ${ }^{273}$ However, the Higuchi law was extended to colloid systems, such as emulsions. ${ }^{274,275}$ The K-P equation organizes the cases of the drug release from a dosage form, and the cumulative drug amount, $\mathrm{M}_{\mathrm{t}}$, released at time $\mathrm{t}$ is given by the Equation 4.3:

$$
\mathrm{M}_{\mathrm{t}} / \mathrm{M}_{0}=\mathrm{kt}^{\mathrm{n}} \text { up to } \mathrm{M}_{\mathrm{t}} / \mathrm{M}_{0} \leq 0.07
$$

Equation 4.3

where $\mathrm{M}_{0}$ is the initial amount of drug in the liposomes at time $\mathrm{t}=0, \mathrm{k}$ is the release constant and $\mathrm{n}$ is the release exponent, indicative of the drug release mechanism. If $\mathrm{n}=1$, the release is zero order. If $\mathrm{n}$ is between 0.5 and 1 , the release follows an anomalous transport (non-Fickian) mechanism, which is controlled by factors other than diffusion, including the drug encapsulation matrix and liposome membrane structure. If $n=0.5$, the drug release is controlled by Fickian diffusion, and the drug release can be modeled using Higuchi's law. All the factors influencing the relationships between $\mathrm{M}_{\mathrm{t}}$ and $\mathrm{t}$ in Equation 4.2 are represented by the release rate constant $\mathrm{k}$ in Equation 4.3. The rate limiting steps in MPS release include (I) MPS diffusion to the inside surface of the liposome and II) MPS diffusion through the lipid bilayer.

\subsubsection{Influence of PEG Incorporation on the Release Mechanism}

The percentage cumulative MPS release $\left.\left(\mathrm{M}_{\mathrm{t}} / \mathrm{M}_{\infty}\right) \times 100 \%\right)$ from liposomes containing PEGylated lipid was shown in Figure 4.8. As shown in Table 4.1, good correlation was obtained between experimental data and the K-P equation with $\mathrm{r}^{2}$ values greater than 0.97 . The release rate constant, $\mathrm{k}$ and the release exponent, $\mathrm{n}$ were obtained from the intercept and slope, respectively, of the plot of $\operatorname{Ln}\left(\mathrm{M}_{\mathrm{t}} / \mathrm{M}_{\infty}\right)$ versus $\mathrm{Ln}(\mathrm{t})$. It was found that the value of $n$ increased with PEG content in the liposomes. It is concluded that Fickian diffusion $(n=0.5)$ is the main release mechanism for liposomes with less than or equal to 2.5 mole\% DSPE-PEG 2000 in liposome formulations. While the release mechanism followed non-Fickian diffusion mechanism at DSPE-PEG 2000 greater than or equal to $5 \mathrm{~mole} \%$. This data shows that MPS release is controlled by the interfacial structure of the liposomes with tethered PEG chains at the surface.

In this case, liposomes can be considered as reservoir systems, where the drug was surrounded by a barrier, ${ }^{276}$ e.g. MPS molecules in liposomes composed of lipid membrane and PEG chains.Narasimhan et al. ${ }^{277}$ suggested that diffusion through the polymer (such as PEG) is the rate-limiting step. If the Fickian diffusion law is obeyed for MPS release from liposomes, the diffusion rate is proportional to: (I) membrane permeability, therefore, DSPE-PEG 2000 in liposomes can reduce the diffusion rate because HSPC bilayers are less rigid than PEG layers, (II) thickness of the bilayer; hence MPS diffusion from PEGylated liposomes. PEGylated liposomes exhibited high release rates. The phospholipid membrane generally is $\sim 2-4 \mathrm{~nm},{ }^{278}$ whereas the PEGylated coat of the liposome is $\sim 5 \mathrm{~nm}$, (III) drug concentration gradient across the liposome bilayer leads to 


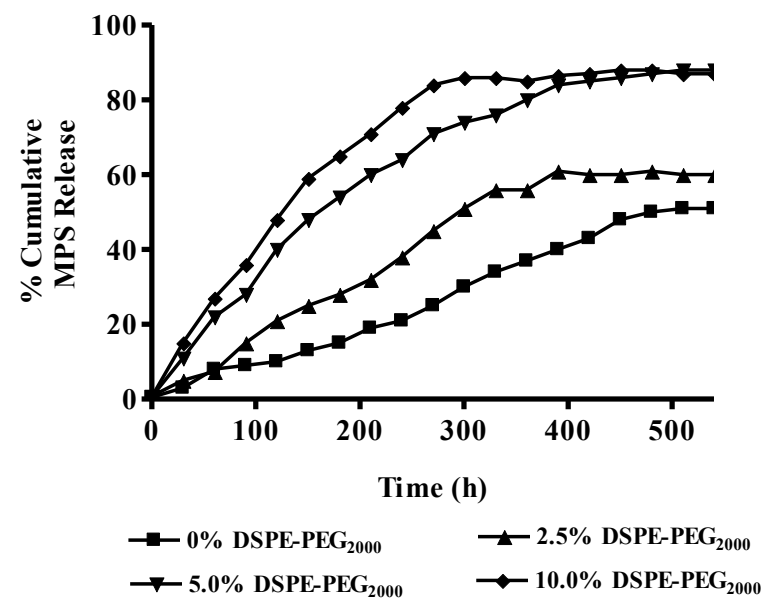

Figure 4.8 Graph depicting the MPS release from liposome formulations containing different fractions of DSPE-PEG 2000 .

The release studies were performed at $25^{\circ} \mathrm{C}$ using dialysis cassettes (10,000 Da MWCO). All the values are a mean \pm SEM of $n=3$ samples.

Table 4.1 Parameters in the K-P equation for MPS release kinetics from MPS-L.

\begin{tabular}{lcccc}
\hline DSPE-PEG 2000 mole $\%$ & $\mathrm{n}$ & Release mechanism & $\mathrm{k}\left(\mathrm{min}^{-\mathrm{n}}\right)$ & $\mathrm{r}^{2}$ \\
\hline 0 & 0.51 & Fickian diffusion & 0.12 & 0.98 \\
2.5 & 0.53 & Fickian diffusion & 0.05 & 0.98 \\
5 & 0.68 & Non-Fickian diffusion & 0.3 & 0.99 \\
10 & 0.71 & Non-Fickian diffusion & 0.01 & 0.97 \\
\hline
\end{tabular}


a high diffusion rate for high MPS encapsulation and (IV) surface area of liposomes, i.e. size of the liposomes.

Silvander et al. ${ }^{279}$ indicated that the PEGylated lipids impose restrictions on the molecular motion in the outer as well as the inner parts of the hydrocarbon region of the lipids. PEGylated lipids may cause an increased packing order at the water/hydrocarbon interface. At $5 \mathrm{~mole} \%$, both diffusion and PEGylated surfaces of liposomes controlled MPS release. The release rate constant, $\mathrm{k}$ decreased dramatically as the PEG was incorporated, and $\mathrm{k}$ values were not significantly different when the PEG-lipid in liposomes was $>5$ mole $\%$. The MPS release from liposomes with PEGylated lipid less than $5 \mathrm{~mole} \%$ followed a $\mathrm{t}^{0.5}$ dependence (Higuchi law) rather than a $\mathrm{t}^{0.7}$ law (for PEGlipid $>5$ mole $\%$ in liposomes).

\subsubsection{Influence of Mean Size of Liposomes on the Release Kinetics}

Liposome diameter has been reported to influence the pharmacokinetics and disposition of a drug, such as doxorubicin into the RES. ${ }^{280}$ The MPS release kinetics from PEGylated liposomes of different sizes is shown in Figure 4.9. The data was fit by the Higuchi law, which showed a good correlation of this model to MPS release from PEGylated liposomes $\left(r^{2}\right.$ is $\left.0.97-0.99\right)$. Higuchi law shows that the surface area of liposomes exposed to the release medium is part of the release rate constant, $\mathrm{k}$ calculated from the relationship between the cumulative drug release amount and $t^{0.5}$. Therefore, MPS release is related to the size/surface area of liposomes. The MPS release rate increased with decreasing liposome size because the total surface area (TSA) of liposomes was increased. TSA is equal to the average surface area (S) of liposomes multiplied by the number of liposomes in suspension, which is equal to the inverse of the volume of a liposome (V). Therefore, TSA is proportional to (A/V), i.e. $\left(4 \pi r^{2}\right) /\left(4 / 3 \pi r^{3}\right)=3 / r(r$ is the average radius of PEGylated liposomes). The MPS release rate constant as a function of $3 / \mathrm{r}$ is shown in Figure 4.10. An increase in the surface area of liposomes led to an increase in $\mathrm{k}$ values due to the increase in total surface area exposed to the release medium, and also led to an increase in MPS diffusion from liposomes. For a PEGylated liposome, Higuchi law is followed during the MPS release, and the release rate constant is proportional to the inverse of liposome radius. In Figure 4.10, the relationship is, $\mathrm{k}=$ $(3.9 / \mathrm{r})+0.0135$. When $\mathrm{r} \rightarrow \infty$, $\mathrm{k}$ is $0.0135 \mathrm{~min}^{-0.7}$, which is the situation of MPS release from a flat surface.

\subsubsection{Influence of Temperature on the Release Kinetics}

Temperature of the release medium may alter the viscosity of the liposome suspension, and thereby change the solubility and diffusion rate of drugs incorporated in liposomes. ${ }^{281}$ Figure 4.11 shows that MPS release as a function of temperature of the medium. Release data was fit to Equation 4.3 with $\mathrm{n}=0.7$ for PEGylated ( 5 mole\%) liposomes. As shown in Table 4.2, temperature $\left(25 \mathrm{vs} .37^{\circ} \mathrm{C}\right)$ has a significant effect on MPS release. An increase of temperature increases the value of the diffusion coefficient, 


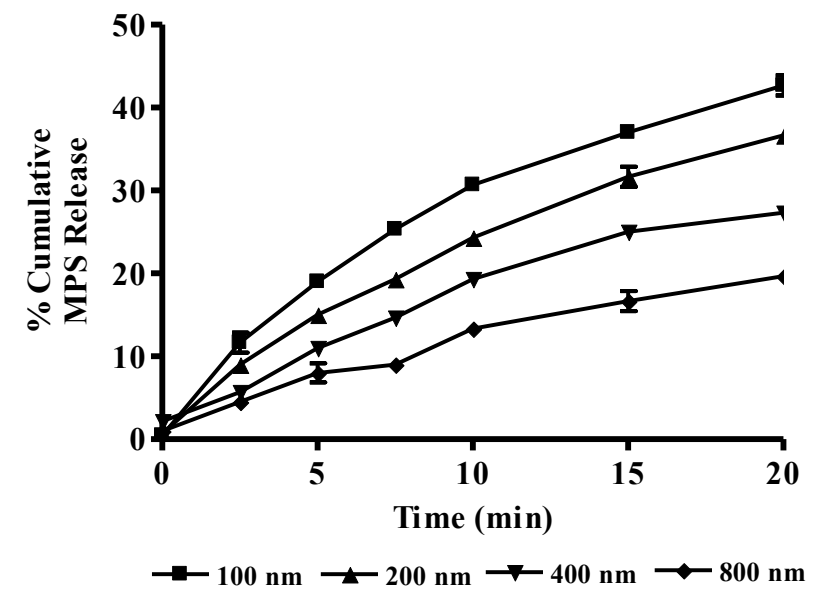

Figure 4.9 Graph depicting the MPS release from PEGylated liposome formulations with mean diameters of 100, 200, 400 and $800 \mathrm{~nm}$.

The total lipid content in all the formulations was $27.2 \mathrm{mM}$, and the studies were carried at the same stirring speed $(50 \mathrm{rpm})$ at $25^{\circ} \mathrm{C}$. All the values are a mean $\pm \mathrm{SEM}$ of $\mathrm{n}=3$ samples.

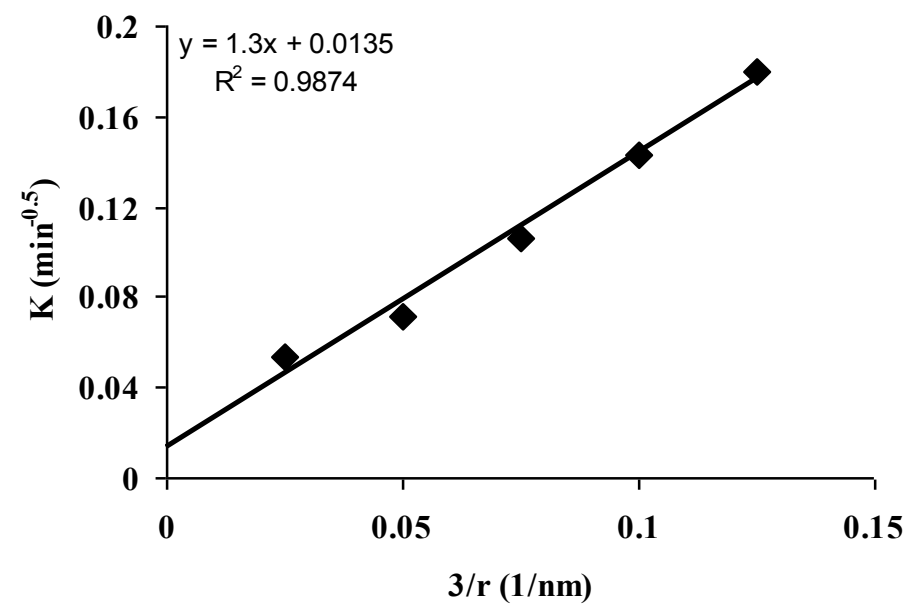

Figure 4.10 Graph depicting the MPS release rate constant of the liposome formulations with varied mean diameter, calculated from K-P equation.

The release rate constant is related to radius of the liposomes. The rate constants were obtained from the slopes of the MPS release from different sized liposome formulations. 


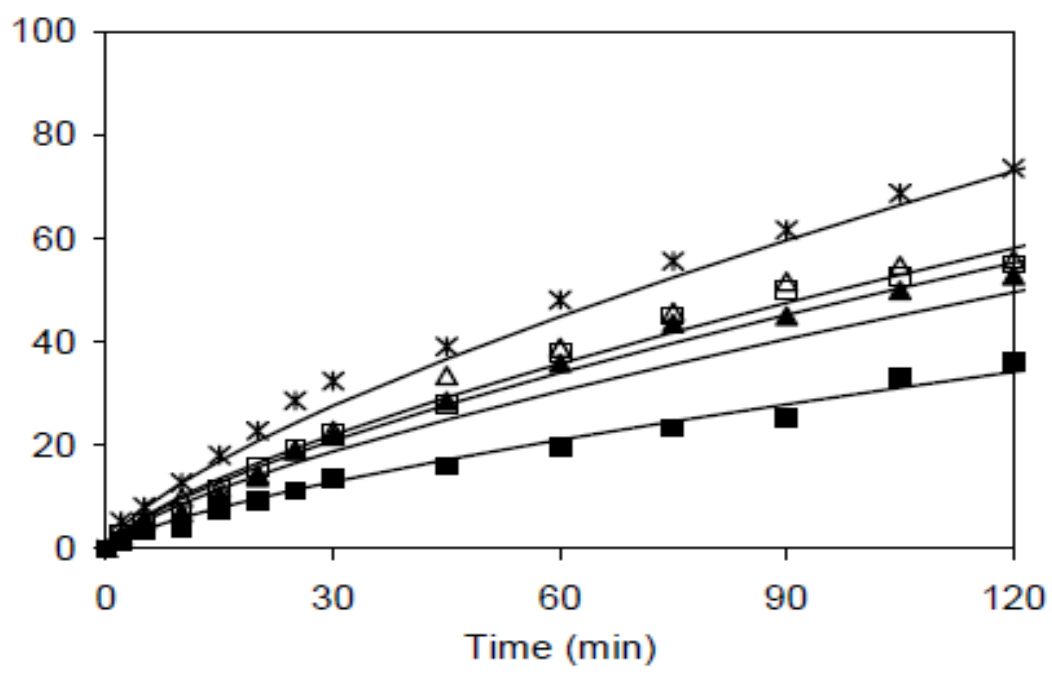

Figure 4.11 MPS release rate from PEGylated liposomes stored at different temperatures $4(\mathbf{\square}), 25(\square), 30(\Delta), 37(\boldsymbol{\Delta})$ and $40^{\circ} \mathrm{C}(*)$.

The MPS release rates were fit to K-P equation.

Table 4.2 Values of $\mathrm{k}$ from the $\mathrm{t}^{0.7}$ law for MPS-L at different temperatures.

\begin{tabular}{lc}
\hline Temperature $\left({ }^{\circ} \mathrm{C}\right)$ & $\mathrm{k}\left(\mathrm{min}^{-0.7}\right)$ \\
\hline 4 & 0.02 \\
25 & 0.04 \\
30 & 0.04 \\
37 & 0.08 \\
40 & 0.12 \\
\hline
\end{tabular}

The release study was conducted at $\mathrm{pH} 7.4$ and, the buffer has no added salt. 
$\mathrm{D}$, which relates the diffusion process to temperature (T) and viscosity $(\eta),{ }^{282}$ In Equation 4.4, kB is Boltzmann's constant and $r$ is the liposome radius. The temperature effect on the D value is also described by Equation 4.5.

$$
\begin{aligned}
& \mathrm{D}=\mathrm{kBT} /(6 \pi \eta \mathrm{r}) \\
& \mathrm{D}=\mathrm{D}_{0} \mathrm{e}^{(-\mathrm{Ead} / \mathrm{RT})}
\end{aligned}
$$

\section{Equation 4.4}

Equation 4.5

Where $\mathrm{D}_{0}$ is a constant, Ead is the activation energy for the diffusion process $(\mathrm{J} / \mathrm{mol}), \mathrm{R}$ is the universal gas constant $(\mathrm{J} / \mathrm{mol} \mathrm{K})$ and $\mathrm{T}$ is the absolute temperature. From Equation 4.4, $\mathrm{D}$ increases with increase in $\mathrm{T}$, which is in agreement with the experimental value. As shown in Figure 4.11, over the temperature range, 4 to $40^{\circ} \mathrm{C}$, MPS release showed Arrhenius behaviour. The activation energy, Ea for MPS release through the liposome bilayer was calculated from the temperature dependence of the release rate constant using the Arrhenius equation. ${ }^{283}$ For PEGylated liposomes, the diffusion process is not the only mechanism and Ea is larger than Ead. However, PEG chains impeded the drug diffusion.

\subsubsection{Summary for Passive Loading Strategy}

MPS encapsulation efficiency was $\sim 8 \% \mathrm{w} / \mathrm{v}$ using passive loading process. The K-P equation $(n=0.7)$ and the Higuchi model $(n=0.5)$ provided good fits for the experimental data of MPS release/leakage from PEGylated liposomes at PEGylated lipid concentrations, $<2$ and $>5$ mole $\%$, respectively. The high surface area of small liposomes led to increased MPS release. Changes in $\mathrm{pH}$ and salt concentration significantly influenced the MPS release rate, indicating that electrostatic forces between liposomes or between liposome and MPS molecules affect the drug release. In contrast to the assumption that PEG incorporation into the liposome formulation reduces MPS release rate, PEGylation increased the MPS release rate. A high molar ratio of PEGylated lipid in the liposome formulations resulted in increased release rate. In addition, the release mechanism was transformed from diffusion control to interfacial control based on the K$\mathrm{P}$ equation. These findings may be utilized for the improvement of the formulation, and gives an insight into the actions of PEGylated liposomes in the body.

\subsubsection{Active Loading Strategies}

\subsubsection{Dynamic Light Scattering (DLS) Studies for Particle Size and Zeta Potential}

The average diameter and zeta potential of extruded liposomes were $120 \pm 13 \mathrm{~nm}$ and $27 \pm 4.7 \mathrm{mV}$, respectively as determined by DLS studies, which indicate the formation of small and stable liposomes. MPS-L ${ }^{\text {cRGD }}$ were stable at $4{ }^{\circ} \mathrm{C}$. 


\subsubsection{Encapsulation of MPS by the Use of Salt Gradients}

MPS liposomes loaded with gradients of calcium acetate showed a higher EE than those loaded by sodium salts (Figure. 4.12). The liposomes showed a constant particle size and low polydispersity index during the loading process (data not shown). The EE declined in the line: calcium $>$ sodium $>$ ammonium. Among the acetate salts, only calcium showed a high MPS loading. All salt gradients resulted in active MPS loading, when compared to the passive loading, which is calculated by the ratio of encapsulated MPS amount to the total MPS concentration.

\subsubsection{Influence of Intravesicular Acetate Concentration on MPS Encapsulation}

Dried lipid was hydrated with various buffers containing 50,100, 200, 300 or $400 \mathrm{mM}$ calcium acetate ( $\mathrm{pH}$ was adjusted to 7.0 with $0.1 \mathrm{~N} \mathrm{NaOH}$ ). The size of the liposomes after extrusion was around 100-120 $\mathrm{nm}$. External acetate was removed using de-salting columns. After incubation of MPS with preformed liposomes at $48^{\circ} \mathrm{C}$, the encapsulation efficiency (EE) increased up to $63 \% \mathrm{w} / \mathrm{v}$ with a [drug/lipid] ratio of 0.4 at an acetate concentration of $200 \mathrm{mM}$ inside the liposomes. The lowest MPS EE (3.1\% w/v) was achieved with $50 \mathrm{mM}$ HEPES buffer, containing no acetate ions. (Figure 4.13)

In the study, a stable $\mathrm{pH}$ gradient over the loading period is maintained in situ by generation of acetate gradient, i.e. by a high intraliposomal acetate concentration and an acetate ion-free, extraliposomal buffer. Due to the acetate gradient, the $\mathrm{pH}$ value decreases outside the liposome from $\mathrm{pH} 4.3$ to a lower $\mathrm{pH}$, even though the extraliposomal buffer was set to a $\mathrm{pH}$ of 4.3. The in situ generation of the $\mathrm{pH}$ gradient from the higher permeation coefficient of acetic acid caused basification of the liposome interior. In the absence of an acetate gradient, there is no decrease in extraliposomal $\mathrm{pH}$ value. Instead, the internal $\mathrm{pH}$ adjusts to that of the outer $\mathrm{pH}$. Due to de-protonation and precipitation of MPS, high encapsulation efficiency was achieved. Due to the interaction of MPS with calcium ions, negatively charged MPS precipitates with calcium ions to form a gel-like structure within the liposomes. ${ }^{187,195,284}$ The solubility studies showed consistently poor solubility of MPS in a $200 \mathrm{mM}$ acetate solution in the investigated $\mathrm{pH}$ range of values close to the $\mathrm{pKa}$ of MPS $(\mathrm{pKa}=4.3)$, where solubility drops to almost zero.

\subsubsection{MPS Encapsulation via Calcium Acetate Gradient with Different Interior pH Values}

Liposomes were prepared with intraliposomal calcium acetate buffer $(200 \mathrm{mM})$ of various $\mathrm{pH}$ values ( $\mathrm{pH} 3,4.3,5,6$ and 7.0). Loading liposomes with MPS via acetate gradient is a new, potent and alternative remote loading method. Loading of MPS by means of salt gradients includes two synergistic effects, both of which result in the fact that MPS can no longer pass the lipid membrane. On one hand, loading is driven by deprotonation and charging of MPS within the liposome, and on the otherhand, by 


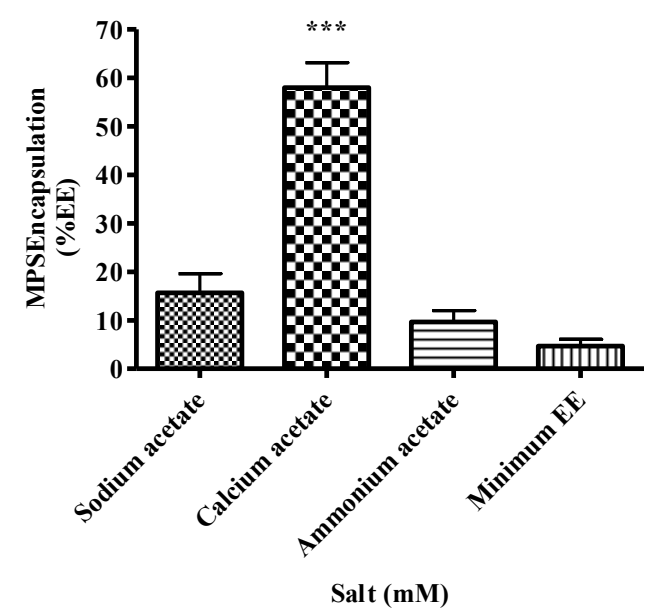

Figure 4.12 Histogram of MPS encapsulation driven by various acetate salt gradients in PEGylated liposomes.

The lipid formulations were prepared with a [drug/lipid] ratio of 0.4. The lipid film (27.2 $\mathrm{mM}$ phospholipid concentration) was hydrated with $200 \mathrm{mM}$ salt solution adjusted to $\mathrm{pH}$ $7.0(\mathrm{n}=3) . * * *$ indicates significant levels at $\mathrm{P}<0.001$ (ANOVA) in comparison with minimum EE.

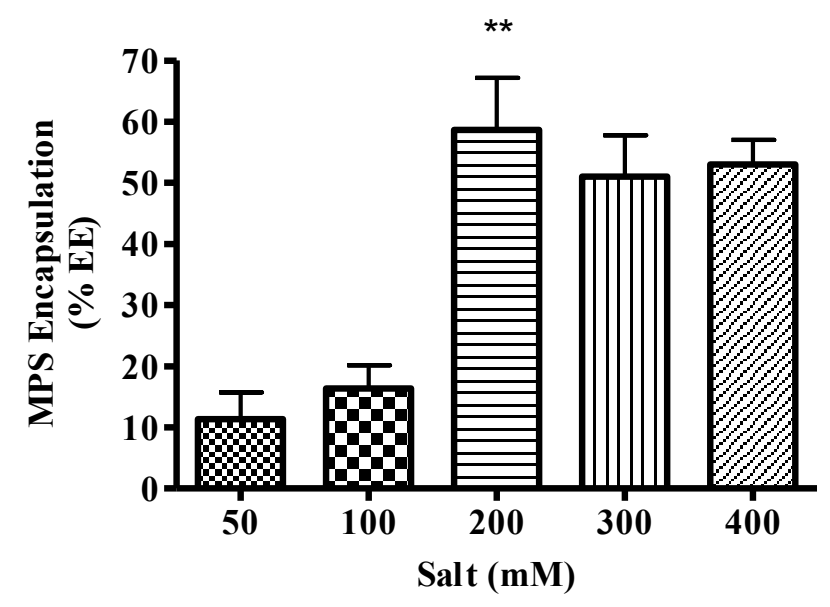

Figure 4.13 Histogram of MPS encapsulation as a function of different ionic strengths of calcium acetate inside the lipoosmes.

All the values are a mean $\pm \operatorname{SEM}(\mathrm{n}=3)$. ${ }^{* *}$ indicates significant levels at $\mathrm{P}<0.01$ (Bonferroni's one way ANOVA) in comparison with minimum EE. 
precipitation of MPS in the hydrophilic interior of the vesicle when the concentration of MPS solubility is exceeded. By means of a stable $\mathrm{pH}$-gradient, MPS is de-protonated in the basic interior of the liposome. When the extraliposomal $\mathrm{pH}$ is low, only a small part of the MPS is de-protonated. The free uncharged acid is then able to pass the membrane so that there is intra and extraliposomal adjustment in the concentration of the free acid of the drug. When the intraliposomal $\mathrm{pH}$ value is higher, the free intraliposomal MPS acid is de-protonated and new extraliposomal, unionized MPS can diffuse into the liposome according to the concentration gradient. Therefore, a "proton pool" outside the liposomes is necessary as a driving force for MPS loading.

As shown in Figure. 4.14, high $\mathrm{pH}$ values resulted in a higher EE of MPS. At $\mathrm{pH}$ values $<4.3$, the solubility of MPS decreases. After protonation of MPS in the liposome aqueous core, the complex is nearly insoluble in the calcium acetate solution. By using calcium acetate gradients with various $\mathrm{pH}$ values, it could be demonstrated that with the synergism of de-protonation and precipitation of MPS, higher encapsulation efficiencies can be achieved.

\subsubsection{Leakage of MPS Liposomes Loaded via Acetate Gradient}

MPS leakage from liposomes was examined under different $\mathrm{pH}$ conditions at $25^{\circ} \mathrm{C}$. These include contact with physiological fluids at $\mathrm{pH} 7.0, \mathrm{pH} 6.0$ of tumor tissues ${ }^{285}$ and $\mathrm{pH} 5.0$ after uptake into endosomes. The drug leakage properties of liposomes, which were loaded via transmembrane acetate gradient differ completely from vesicles, which are loaded with other ion gradients. The MPS release of liposomes, which were loaded via an acetate gradient showed a negligible leakage at low $\mathrm{pH}$, but leakage increased with $\mathrm{pH}$ (Figure 4.15). At pH 6.0 and 7.0, the drug release is influenced by the extraliposomal ratio of negatively charged MPS to neutral MPS. Furthermore, release experiments have been performed with these liposomes at $37^{\circ} \mathrm{C}$ (data not shown). $\mathrm{pH}$ dependent release from the liposomes significantly increased, which might be due to increased MPS solubility and/or membrane fluidity.

Besides efficient loading, the release of the drug is also a critical factor for a drug delivery system. As shown, the release of MPS from liposomes is highly dependent on the extraliposomal $\mathrm{pH}$ value. In this study, $\mathrm{pH}$ values, $7.0,6.0$ or 5.0 similar to the situations in most tissues, tumors and endosomes were chosen to imitate the various $\mathrm{pH}$ environments of the liposome from the point of application to the reaching of the target. In the case of MPS liposomes, a high extraliposomal $\mathrm{pH}$ value results in facilitated MPS release, whereas liposomes are not significantly influenced by the low extravesicular $\mathrm{pH}$ value.

\subsubsection{6 (DSPE-PEG 2000 -maleimide)-cRGD Conjugate Synthesis and Micelle Formation}

(DSPE-PEG 2000 -maleimide)-cRGD conjugate was micellized for later insertion into the preformed liposomes at well defined numbers of targeting ligands. Final product yield 


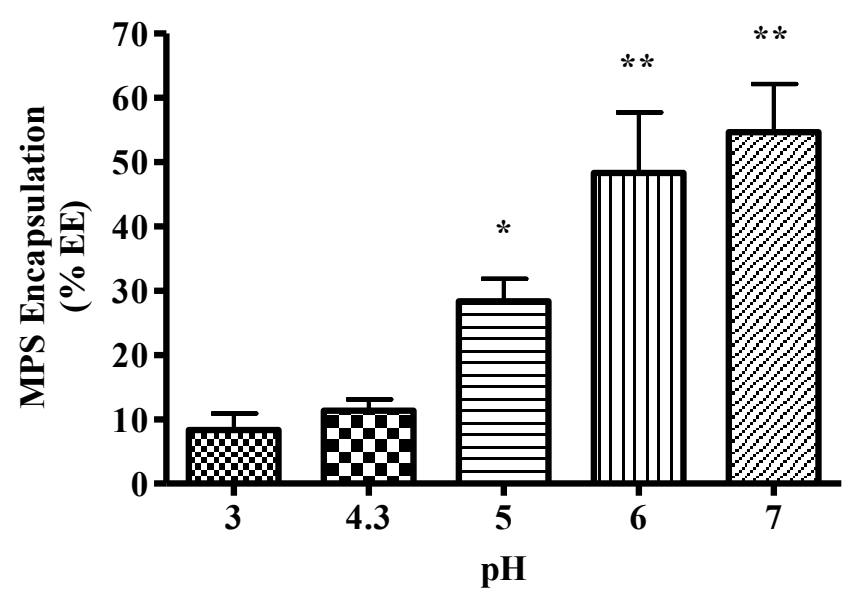

Figure 4.14 Histogram of MPS encapsulation in liposomes as a function of intravesicular $\mathrm{pH}$.

Liposomes were prepared with $200 \mathrm{mM}$ calcium acetate buffer of different intravesicular $\mathrm{pH}(\mathrm{n}=3) .{ }^{*}$ and $* *$ indicate significant levels at $\mathrm{P}<0.05$ and $\mathrm{P}<0.01$, respectively in comparison with MPS encapsulation at $\mathrm{pH} 3.0$ (Bonferroni's one-way ANOVA).

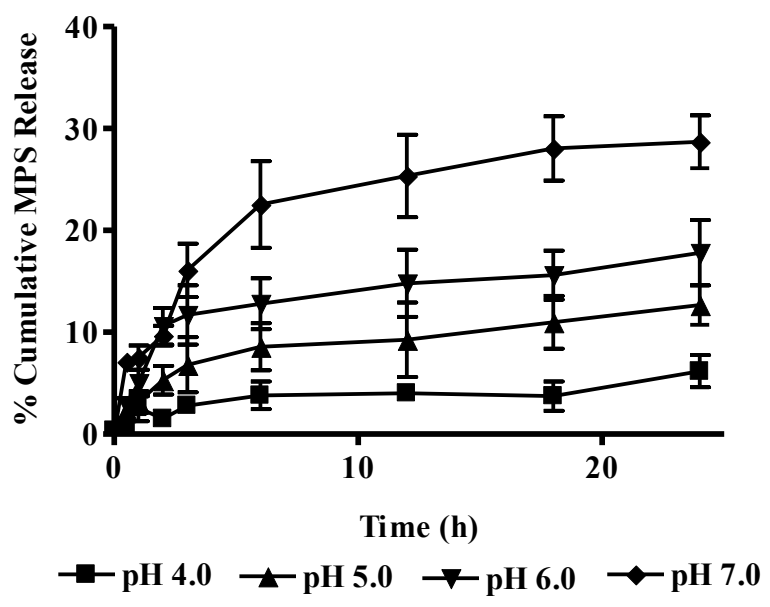

Figure 4.15 MPS release from liposomes as a function of $\mathrm{pH}$ of the external medium at $25^{\circ} \mathrm{C}$.

MPS was encapsulated by calcium acetate gradient. The drug release was analyzed in 50 $\mathrm{mM}$ HEPES, $140 \mathrm{mM} \mathrm{NaCl}$ buffers, $\mathrm{pH} 4.0,5.0,6.0$ and 7.0 at $25^{\circ} \mathrm{C}$. Data points are a mean $\pm \mathrm{SEM}$ of $\mathrm{n}=3$ samples. 
was 34\% (molar), as determined by Ellman reaction and phosphorus content of the lipid. The product was analyzed qualitatively by MALDI-MS. As shown in Figure 4.16, the peak corresponding to the lipid-peptide targeting conjugate was observed at 3458.29 Da. Mass spectra for the starting materials were 586 Da for cRGD-peptide and 2941 Da for DSPE-PEG 2000 -maleimide (data not shown).

\subsubsection{7 (DSPE-PEG 2000 -maleimide)-cRGD Conjugate Insertion into MPS-L}

In order to construct MPS-L bearing defined number of targeting ligands, (DSPE$\mathrm{PEG}_{2000}$-maleimide)-cRGD conjugate was incorporated into the lipid bilayers of MPS-L by the 'post-insertion' technique. Following dialysis, the unincorporated (DSPE-PEG $2000^{-}$ maleimide)-cRGD conjugate was estimated as described elsewhere. The number of cRGD peptides per liposome was determined by subtraction method. From the peptide and phospholipid analysis, the number of targeting ligands intended for insertion was $1500 \mathrm{cRGD}$ peptides per liposome. From the analysis, approximately 680 ligands were inserted into liposomes. This is equivalent to $45.3 \%$ (molar) of the targeting conjugate insertion efficiency.

\subsubsection{Summary for Active Loading Strategy}

The development of MPS-L for treating inflammatory diseases has been a long-sought goal, as evident from numerous studies starting in the 1970s and continuing to this day. However, although much progress has been made, some major limitations and restrictions still prevent the pharmaceutical development of MPS-L formulations for clinical use. From a historical perspective, in the early stage of research related to MPS-L, the main idea was to utilize their lipophilic nature and to encapsulate them in the liposome membrane. This form of encapsulation has encountered a major limitation of stability, since most GCs are not lipophilic enough, as is obvious from their $\log \mathrm{D}$ and mass aqueous solubility. For instance, CHOL, one of the liposome membrane components, has a $\log \mathrm{D}$ of 9.85 at $\mathrm{pH} 7.0$ and mass solubility of $6 \times 10^{-9} \mathrm{gm} / \mathrm{L}$, while hydrocortisone has a much lower $\log \mathrm{D}$ of 1.43 at $\mathrm{pH} 7.0$ and mass solubility of $0.16 \mathrm{gm} / \mathrm{L}{ }^{282}$ Therefore, such GCs are not suitable for "encapsulation" in the liposome bilayer, since upon dilution they will desorb to the aqueous medium. Mishina et al. ${ }^{195}$ demonstrated that $50 \% \mathrm{w} / \mathrm{v}$ of encapsulated methylprednisolone was released to the bulk medium during 10 days of storage. In the present study, obstacles associated with passive loading approach were overcome with active remote loading of MPS.

It seems that the cosmotropic properties of the counter-ion contribute to the solubility of MPS. The salts used for loading, i.e., ammonium, sodium and calcium are all components of the Hofmeister or lyotropic series. ${ }^{286,287}$ This series reflects the ranking of ions according to their binding strength of bulk water and to the concomitant decrease in the hydration of other compounds. The precipitation caused by these compounds is then termed as salt-out effect. This also explains the requirement of the high salt concentration of $200 \mathrm{mM}$ for liposome loading, since this concentration is necessary to withdraw the 


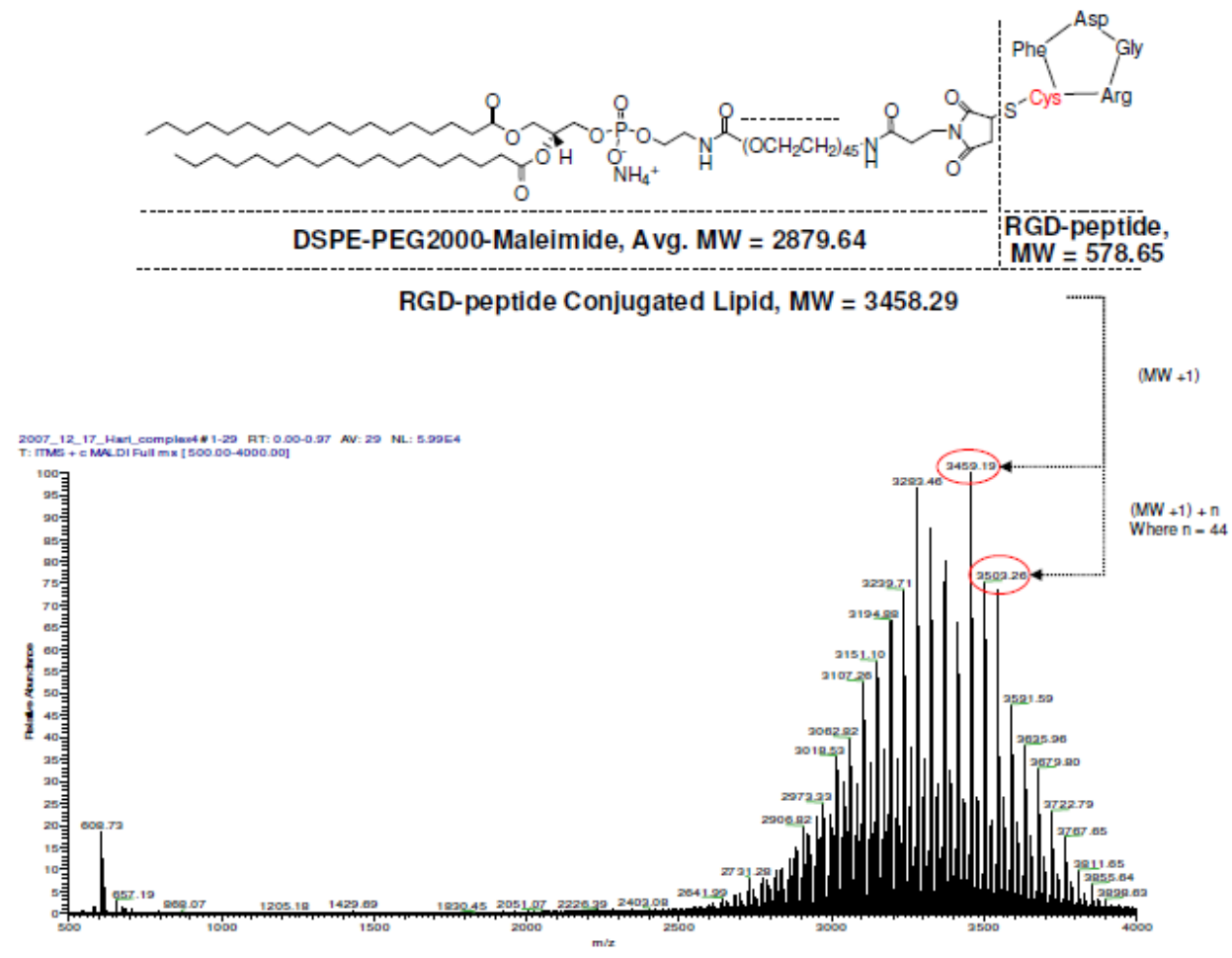

Figure 4.16 MALDI-MS spectra of the (DSPE-PEG 2000 -maleimide)-cRGD-peptide conjugate.

The product was characterized by vMALDI. For an MS scan, high-mass range (m/z 500 4000) and normal scan rate were used. Product spectra were acquired and analyzed using Xcalibur software. MADLI-MS analysis was performed in positive ion mode, and therefore, the mass obtained on the spectra is usually $\mathrm{M}+1$. 
hydration shell of MPS. This salt-out effect plays a role not only for proteins but for amphiphilic substances as well, which has been shown in other studies. ${ }^{288}$ The withdrawal of the hydration shell also explains the self-aggregation of MPS driven by hydrophobic interactions, which is highly dependent on the respective buffer composition.

In conclusion, the active loading process of MPS in liposomes via acetate gradient depends on various factors: intraliposomal salt concentration, water binding potential of the salt, the $\mathrm{pH}$ value in the liposome and the presence of acetate pool. The loading of MPS in liposomes via calcium acetate gradient represents a potent alternative procedure to the methods used to date. It allows fast and effective loading of the MPS into liposomes at $48^{\circ} \mathrm{C}$. Although, there is evident release of MPS at $37^{\circ} \mathrm{C}$, under long term $(3$ months) storage conditions at $4^{\circ} \mathrm{C}$, there is a minimal $(8 \% \mathrm{w} / \mathrm{v})$ release of MPS. We were able to demonstrate that the novel active loading method facilitates dual purposes: I) MPS encapsulation (63\% w/v encapsulation efficiency) and II) high targeting ligand insertion (45.3\% molar) into lipid bilayers. 


\section{CHAPTER 5. PROTECTION OF COLLOIDAL PROPERTIES OF MPS-L ${ }^{\text {CRGD }}$}

\section{$5.1 \quad$ Introduction}

Liposomes have been successfully employed for the encapsulation of a range of synthetic drugs and biologicals. ${ }^{289,290}$ However, the potential application of liposomes as therapeutic tools is still challenged by their inherent physical and chemical instabilities, which can result in increased bilayer permeability, subsequent drug loss and vesicle aggregation/fusion. These instabilities can be stimulated by chemical degradation (e.g. lipid oxidation and hydrolysis) and physical factors, such as heating or freezing. ${ }^{291}$ For instance, changes in the arrangement of phospholipids within the bilayer of small vesicles have been attributed to the loosely packed head-groups and tightly packed alkyl chains in the outer layer, with the opposite arrangement in the inner layer of the bilayer. ${ }^{292}$ This leads to a thermodynamically unstable state, which favors aggregation and/or fusion of the vesicles. Therefore, it is clear that the composition of the lipid bilayer, bilayer-drug interactions and the storage conditions of the liposomes will all influence liposome stability. $^{293-295}$ To circumvent these problems and achieve long term stability of liposomes, lyophilization has been used as an effective approach to render liposomes stable without compromising their physical state or encapsulation capacity. However, lyophilization of liposome systems without appropriate stabilizers will again lead to fusion of liposomes; a factor exploited by the dehydration-rehydration method. ${ }^{296}$ To promote liposomes stability during the lyophilization process, lyoprotectants, including saccharides (e.g. sucrose, trehalose) and aminoacids (e.g. lysine) are employed. ${ }^{297,298}$ A detailed review by Crowe and Crowe ${ }^{295}$ outlines the mechanism of stabilization offered by the saccharides. Briefly, the increase in liposome size and leakage of the encapsulated material in non-lyoprotected liposomes during lyophilization is attributed to two factors: phase separation of lipids around their gel-fluid transition temperature and fusion of the liposome membranes. Thus, aggregation of liposomes could be prevented by the formation of stable boundaries between the liposomes. The ability of lyoprotectants to form these stable boundaries has been attributed to their ability to replace the bound water around the bilayer via interaction with the polar region of the lipid head group (water replacement hypothesis). ${ }^{295,299}$ Alternatively, Koster et al. ${ }^{300}$ proposed the formation of a vitreous layer (glass formation around the bilayer) of the lyoprotectant around the bilayer, which depresses the transition temperature of the phospholipids thus preventing any drug leakage during gel to fluid phase transformations.

The role of various saccharides (glucose, sucrose, trehalose, etc.) has been investigated thoroughly as stabilizing lyoprotectants. ${ }^{250}$ Despite the detailed investigations, these systems have not been fully optimized, and in order to achieve effective lyoprotection and reproducible formulation parameters, high concentrations (30 mole \%) are required. Therefore, the aim of this work is to assess various lyoprotectants, including disaccharides and aminoacids as stabilizing moieties during the lyophilization of liposomes. Crowe et al. investigated the influence of disaccharides and aminoacids as lyoprotectants. ${ }^{301}$ It has been shown that sugars are able to protect membranes during dehydration. In addition, very little has been reported on the ability of sugars to stabilize 
targeted liposomes, whose surface is modified with peptides or proteins. The present study investigates the ability of disaccharides to protect lyophilized peptide or antibody conjugated liposomes. Parameters, such as particle size distribution, drug leakage and gel-to liquid phase transitions after rehydration were analyzed to investigate if the lyophilized formulations could be reconstituted without physico-chemical changes. Also, amino acids were investigated for use as lyoprotectants in simple liposome formulations.

\subsection{Experimental Section}

\subsubsection{Materials}

HSPC1, DSPE-PEG 2000 , DSPE-PEG 2000 -maleimide were purchased from Avanti Polar Lipids (Birmingham, AL). CHOL, arginine, histidine, lysine, trehalose, sucrose and MPS were obtained from Sigma (St. Louis, MO). cRGD peptide was purchased from Peptide International Inc. (Louisville, KY). IgG antibody was purchased from Chemicon (Minneapolis, MN). Acetonitrile was purchased from Fisher Scientific (Suwannee, GA). All chemicals used were of analytical grade.

\subsubsection{Methods}

\subsubsection{Preparation of Liposomes}

MPS encapsulation into liposomes was achieved by transmembrane calcium acetate gradient method. Liposomes were prepared with a lipid composition of HSPC, CHOL, ${ }_{\text {DSPE-PEG }} 2000$ and DSPE-PEG 2000 -maleimide in 50: 45:3: 2 molar ratios. Lipid (27.2 $\mathrm{mM}$ total phospholipids) solution in chloroform-methanol solution $(3: 1 \% \mathrm{v} / \mathrm{v})$ was subjected to vacuum drying at $40^{\circ} \mathrm{C}$ to form a thin dry film, which was hydrated with calcium acetate buffer $(200 \mathrm{mM})$ at $65^{\circ} \mathrm{C}$ to form MLVs. These vesicles were extruded through 200/100 nm sandwiched polycarbonate membrane filters using LIPEX ${ }^{\mathrm{TM}}$ extruder The MLVs were subjected to 6 extrusion passes to obtain liposomes with a mean diameter of 100-120 nm. To create a transmembrane calcium acetate gradient across the liposomes, the extraliposomal calcium acetate buffer was replaced with $0.9 \%$ saline solution by dialysis (10,000 Da MWCO). Followed by generation of calcium acetate gradient, liposomes were mixed and incubated with MPS solution $[19 \mathrm{mM}]$ at $48^{\circ} \mathrm{C}$ for 6 min and then cooled to $4^{\circ} \mathrm{C}$. Unencapsulated MPS and any traces of calcium acetate were removed by dialysis of MPS-L at $4^{\circ} \mathrm{C}$ for $6 \mathrm{~h}$. cRGD peptide-lipid conjugates were inserted into lipid bilayers for formation of targeted liposomes. Detailed procedure for preparation of targeted liposomes was described elsewhere. Empty liposomes were prepared by Bangham method without MPS encapsulation.

\subsubsection{Lyophilization of Liposomes}


Liposomes were mixed with different lyoprotectants (trehalose, sucrose, lysine, histidine and arginine) to obtain [lyoprotectant/lipid] ratios at 2, 4, 6 and 8:1. The liposome suspensions were then subjected to lyophilization. Vials were loaded into a lyophilizer (Virtis Genesis ES25) and run according to the protocols listed elsewhere.

\subsubsection{Determination of MPS Encapsulation and Retention in Liposomes}

The amount of MPS encapsulated in liposomes was determined after dialysis at $4^{\circ} \mathrm{C}$. Purified liposomes were dissolved and suitably diluted with ethanol before analysis. MPS determination was performed by RP-HPLC using a Nova-pak ${ }^{\circledR}$ C-18 column (3.9x150 $\mathrm{mm}, 4 \mu$ ) (Waters, MA). An isocratic method was run with a mobile phase comprised of sodium acetate: acetonitrile in $67: 33 \% \mathrm{v} / \mathrm{v}$. The $\mathrm{pH}$ of the mobile phase was adjusted to 4.8 with glacial acetic acid. The retention time of MPS was between 5-6 min with absorbance maximum at $250 \mathrm{~nm}$.

\subsubsection{DLS Studies for Particle Size and Zeta Potential}

Mean diameter and zeta potential of liposomes were evaluated by DLS studies as described elsewhere.

\subsubsection{Transmission Electron Microscopy}

Morphology of liposomes was determined by negative staining of the SUV specimens on carbon support films by the single droplet procedure. $2 \% \mathrm{w} / \mathrm{v}$ aqueous solution of uranyl acetate ( $\mathrm{pH}$ 6.5) was employed to stain the specimens.

\subsubsection{Differential Scanning Calorimetry (DSC)}

The gel-to-liquid phase transition temperature of the undiluted vesicles in liposome suspension was determined using DSC. Thermograms were obtained using a Q2000 series DSC (TA Instruments, New Castle, DE). Samples were run at $10^{\circ} \mathrm{C} / \mathrm{min}$ from $45^{\circ} \mathrm{C}$ to $80^{\circ} \mathrm{C}$. Q2000 series software was used for data analysis. The first of three scans of each sample $(n=3)$ was used for data analysis.

\subsubsection{Stability Studies of Lyophilized Liposomes}

To investigate the stability offered by the addition of lyoprotectants, lyophilized liposomal formulations were tested for their particle size and MPS retention for a period of 4 weeks. The lyophilized formulations were stored at $25^{\circ} \mathrm{C}$. The samples were then hydrated and drug leakage was studied. 


\subsubsection{Moisture Content Analysis using Karl Fisher Titration}

Residual moisture content of the lyophilized cakes was measured using the Karl Fisher titration method. ${ }^{302}$ A minimum of $100 \mathrm{mg}$ of pulverized cake was dispersed into an anhydrous methanol bath and titrated with Karl Fisher reagent (Acros, NJ).

\subsubsection{Statistical Analysis}

Statistical analysis was carried out by analysis of variance followed by Bonferroni t-test using GraphPad Prism ${ }^{\mathrm{TM}}$ software.

\subsection{Results and Discussion}

\subsubsection{Disaccharides as Lyoprotectants: Evaluation of Appearance of MPS-L ${ }^{\text {CRGD }}$}

In the present study, MPS- $\mathrm{L}^{\mathrm{CRGD}}$ were lyophilized in the presence of sucrose or trehalose at various concentrations. The lyophilized formulations were examined by visual inspection. A uniform cake matrix was obtained after lyophilization of MPS- ${ }^{\mathrm{CRGD}}$ in the presence of trehalose or sucrose at concentrations ranging from 200 to $400 \mathrm{mM}$ or at [lyoprotectant/lipid] ratios, $4: 1$ or 8:1. At lower concentrations $(<100 \mathrm{mM})$, a collapse of the cake was observed indicating that these concentrations of sugars were insufficient to prevent aggregation of MPS- ${ }^{\mathrm{CRGD}}$. This collapse is illustrated in Figure $\mathbf{5 . 1}$ with pictures of cakes containing $400 \mathrm{mM}$ and $50 \mathrm{mM}$ trehalose. The collapse was more pronounced for the formulations containing sucrose (data not shown). Formulations containing 200 $\mathrm{mM}$ trehalose and $400 \mathrm{mM}$ sucrose were readily rehydrated, and the process occurred within 10 min with no visual sign of aggregates (Figure 5.2). Formulations with lower concentrations of sugar were rehydrated more slowly, and the formulations with the lowest concentrations showed signs of aggregates even hours after rehydration. These results suggest that with sufficient amounts of trehalose or sucrose, it is possible to lyophilize MPS- $\mathrm{L}^{\mathrm{CRGD}}$ and create a stable dry cake. Furthermore, insufficient amounts of lyoprotectant cause slow rehydration. A successful lyophilization should enable the product to rapidly reabsorb solvent, and restore the substance to its original state.

\subsubsection{Effect of Lyoprotectants on Particle Size}

The particle size of MPS- ${ }^{\text {CRGD }}$ was analyzed to determine which sugar concentration was sufficient to preserve the average particle size upon lyophilization. The average particle size of MPS-L ${ }^{\mathrm{CRGD}}$ prior to lyophilization was $120 \pm 13 \mathrm{~nm}$. The average particle 


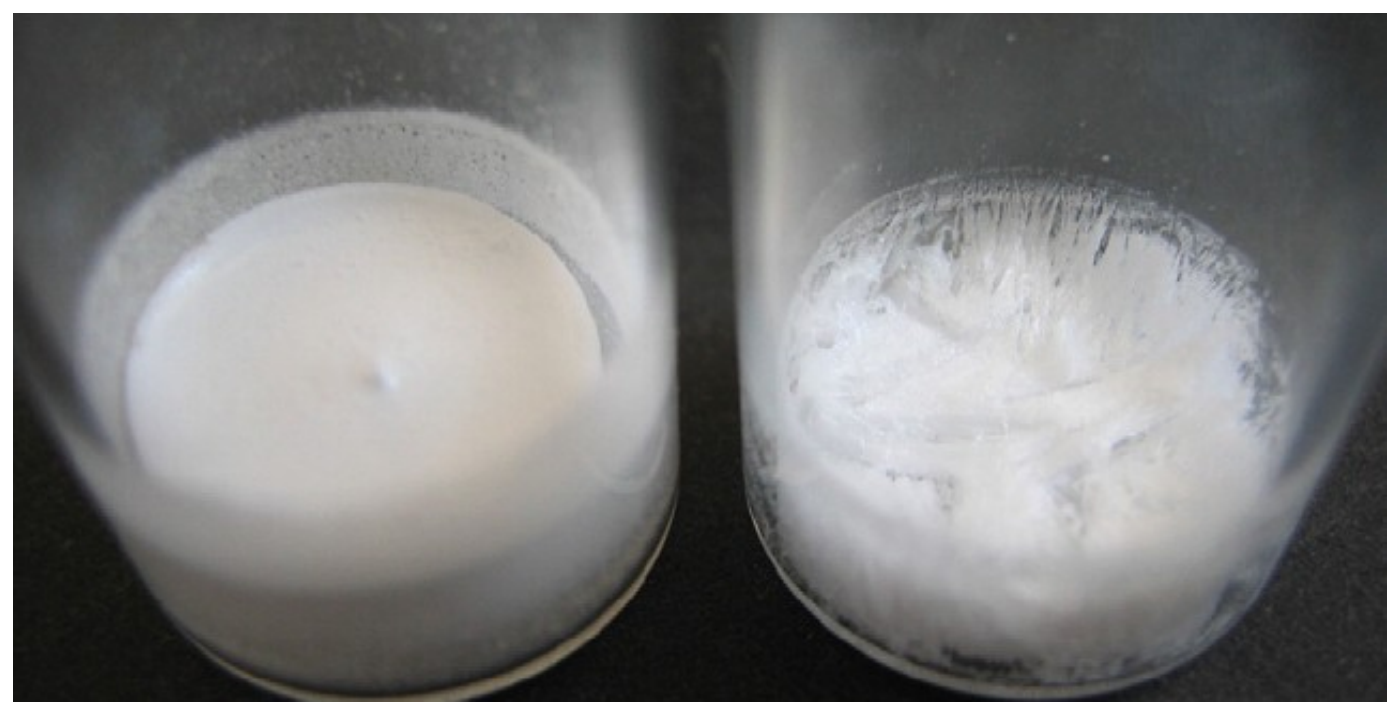

Figure 5.1 Representative samples of the MPS- $L^{\text {cRGD }}$ lyophilized with high (400 mM; left) and low (50 mM; right) concentrations of trehalose.

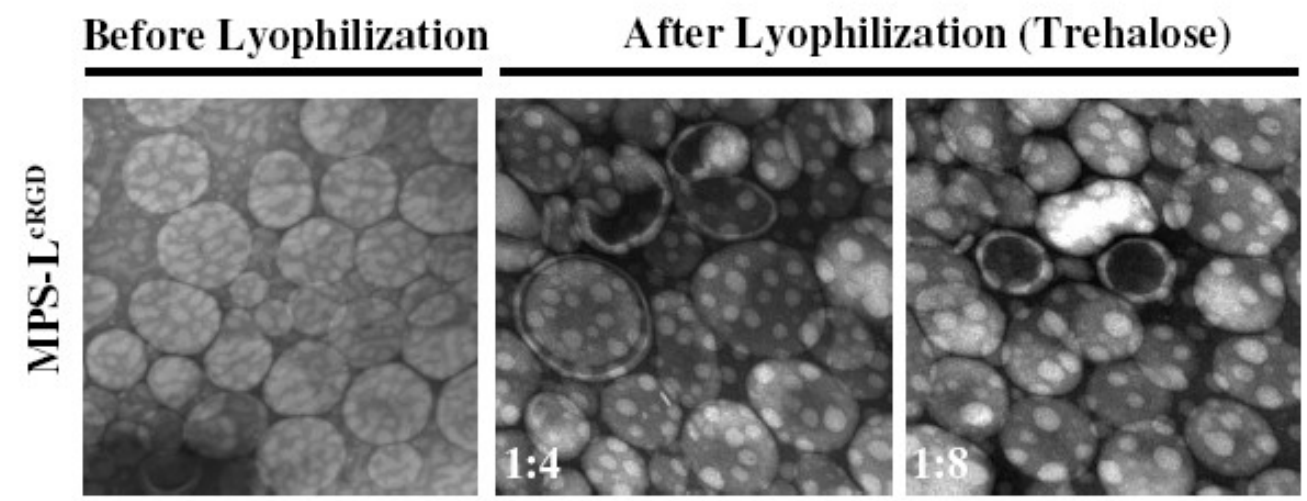

Figure 5.2 Transmission electron micrographs ofMPS- $\mathrm{L}^{\mathrm{cRGD}}$ before and after lyophilization (i.e. reconstituted) with trehalose.

Micrographs depict the morphology of the targeted liposomes lyophilized with $200(1: 4)$ and $400(1: 8) \mathrm{mM}$ of trehalose. 
size of the lyophilized formulations was measured $10 \mathrm{~min}$ after rehydration. The formulations with above $200 \mathrm{mM}$ trehalose regained their normal particle size after 10 min of rehydration $(\mathrm{P}>0.01)$. This was not the case for the formulations with lower concentrations of trehalose indicating that these liposomes were not fully rehydrated or that the membrane structures were not preserved during lyophilization. For sucrose, only at a concentration of $400 \mathrm{mM}$ sucrose, rehydration of the MPS-L ${ }^{\text {CRGD }}$ took place in 10 min. The particle size increase of the lyophilized formulations containing less than 100 $\mathrm{mM}$ sugar was 8-12 times over the original size. At low levels of sugar concentrations, not even $24 \mathrm{~h}$ of rehydration resulted in a normal particle size suggesting that aggregation is irreversible. Since MPS- $\mathrm{L}^{\text {cRGD }}$ is a monodisperse formulation, it was important to analyze if any changes in the particle size distribution could be detected.

As illustrated in Figure 5.3, MPS-L ${ }^{\text {cRGD }}$ with $\geq 200 \mathrm{mM}$ trehalose or $400 \mathrm{mM}$ sucrose, the particle size distribution did not change suggesting that the size distribution was preserved upon lyophilization. Formulations containing $400 \mathrm{mM}$ trehalose or sucrose were found to be more monodisperse than MPS-L ${ }^{\text {cRGD }}$ lyophilized formulations with low sugar concentrations. The results thus show that at trehalose concentrations $\geq 200 \mathrm{mM}$, the formulation can be reconstituted within 10 min without change in average particle diameter as well as particle size distribution, whereas sucrose only preserves the particle size at the highest concentration $(400 \mathrm{mM})$. The results also confirm the conclusion from the visual inspections, that much more sucrose than trehalose is needed to obtain a quick rehydration of lyophilized formulations.

\subsubsection{Effect of Lyoprotectants on the Thermotropic Phase Behaviour of HSPC before} Lyophilization

The thermotropic phase behaviour of HSPC in the presence of trehalose or sucrose was determined before lyophilization to investigate the effect of sugars on the liposome dispersions. The thermograms obtained are shown in Figure 5.4. The phase transition temperature for $\mathrm{HSPC}$ was observed at $52.62 \pm 0.02^{\circ} \mathrm{C}$. Low concentration trehalose slightly increased the Tm to $54.02 \pm 0.08^{\circ} \mathrm{C}$ in non-lyophilized MPS-L ${ }^{\mathrm{CRGD}}$ formulation. There was no apparent difference between Tm of the formulations containing different concentrations of trehalose. Addition of sucrose also did not affect the main gel-to-liquid phase transition temperature of HSPC. However, at $\geq 100 \mathrm{mM}$ trehalose, a shoulder was observed on the high temperature side of the peak, which developed into a distinct peak at $67.68^{\circ} \mathrm{C}$ with $400 \mathrm{mM}$ trehalose. The same pattern was observed with sucrose, though not so pronounced. The small increase in Tm for the formulations containing trehalose and the shoulder on the high temperature side of the peak could be explained by direct interaction. ${ }^{303}$ Direct interaction of trehalose with the polar head-group of the lipids could cause the formation of a depletion layer around the liposomes in which the concentration of water is lower than in the bulk phase. The polar head-groups of trehalose, with hydroxyl groups are available for hydrogen bonding. HSPC of MPS-L ${ }^{\text {cRGD }}$ would therefore have this sugar available on the membrane surface for interaction with cosmotropic molecules like trehalose and sucrose. The cosmotropic effect can cause a destructuring of the H-bond network of bulk water, which affects the dynamic properties of 

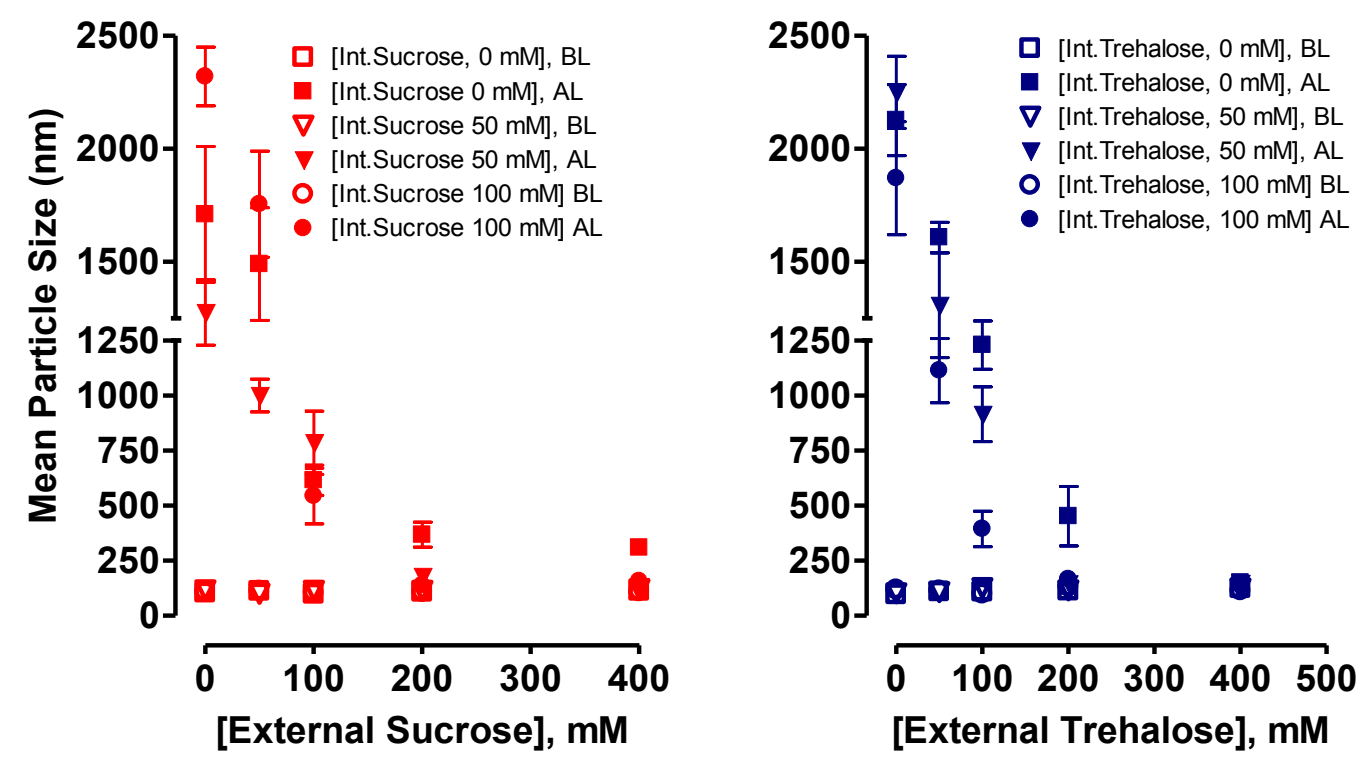

Figure 5.3 Mean diameter of MPS- $\mathrm{L}^{\mathrm{cRGD}}$ as a function of lyoprotectant concentration.

MPS-L ${ }^{\text {cRGD }}$ formulations containing sucrose (red) and trehalose (blue) before (open symbols) and after (closed symbols) lyophilization. All the formulations are a mean \pm SEM, $n=2$ samples. AL indicates after lyophilization and $B L$ indicates before lyophilization. 

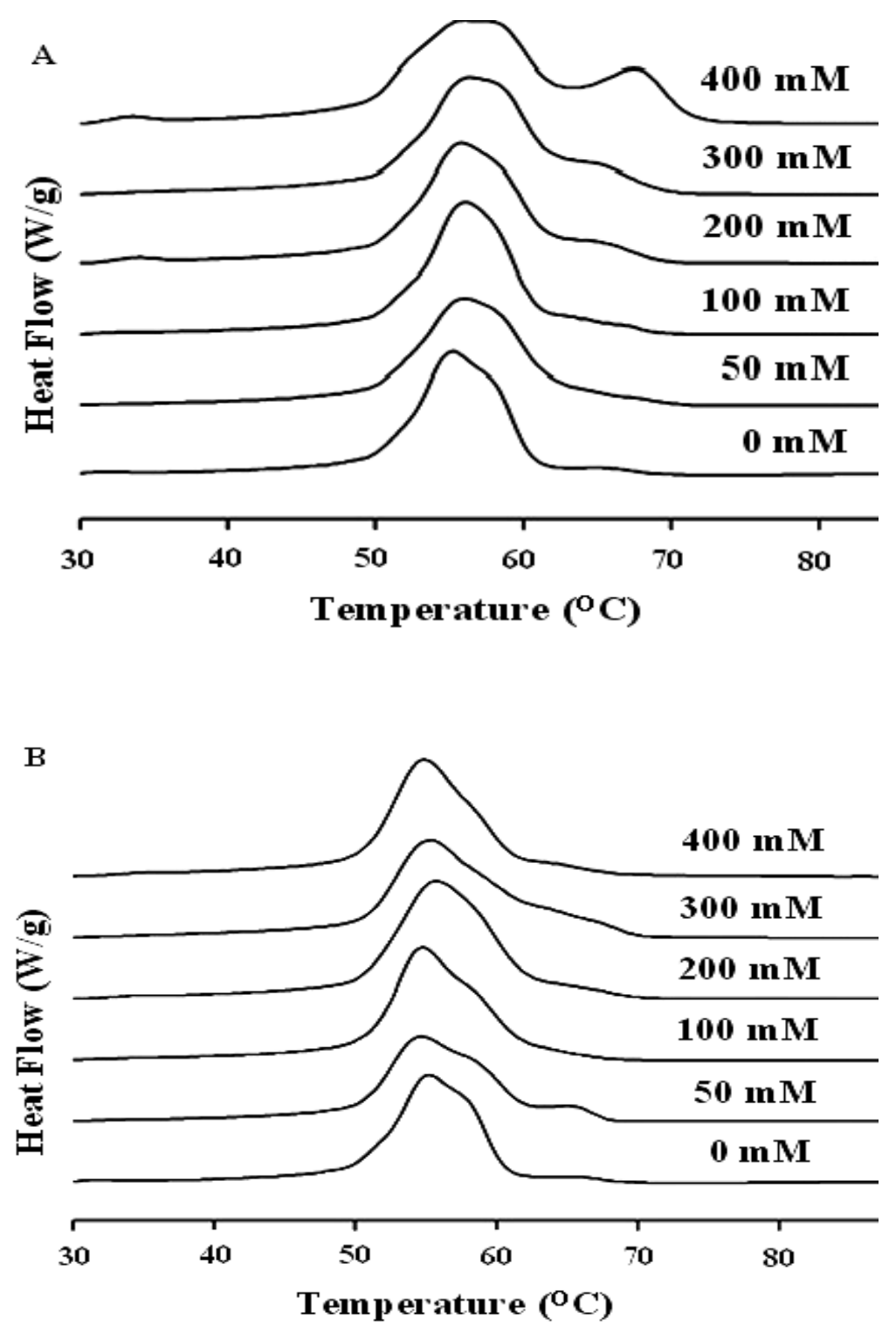

Figure 5.4 Thermograms of MPS-L ${ }^{\text {cRGD }}$ containing increasing concentrations of trehalose (A) and sucrose (B) prior to lyophilization. 
water and reduces the amount of water in the solvation layer surrounding the liposome membranes. ${ }^{304,305}$ This could cause the Tm of the liposome membrane to change due to lower flexibility and higher thermal stability, and thus explains the shoulder on the high temperature side of the peak. ${ }^{304}$ Another explanation for the shoulder on the high temperature side of the peak could therefore be that a phase separation in HSPC local domains with high concentrations of highly ordered HSPC with elevated phase transition temperatures. This phase separation would lead to a lowering of the gel-to-fluid phase transition of these areas. No reduction of Tm was observed suggesting that phase separation cannot explain the shoulder on the high temperature side of the peak. More investigations are required to explain this behaviour further.

\subsubsection{Effect of Lyoprotectants on the Thermotropic Phase Behaviour of HSPC after} Lyophilization

A number of studies have been conducted to examine the interactions of trehalose with the phospholipid membrane at various hydration levels; there is a lack of data pertaining to how the interactions present in the hydrated state translate into those in the dehydrated state. In previous sections, we have examined the effects of trehalose on the transition behavior of HSPC before lyophilization in the presence of sugars. In this section, we examine how these interactions are manifest upon lyophilization. Upon dehydration, the phase transition temperature of HSPC increases from $52^{\circ} \mathrm{C}$ to $83^{\circ} \mathrm{C}$. Removal of water leads to a decrease in the head group spacing of the lipids, thus allowing for increased van der Waals interactions between the lipid hydrocarbon chains. ${ }^{306}$ If the membrane (in the liquid crystalline phase) is dried in the presence of trehalose, the contraction of the phospholipids is avoided and the Tm decreases considerably. ${ }^{307}$ Figure $\mathbf{5 . 5}$ shows the thermograms for the lyophilized liposomes $(\sim 100 \mathrm{~nm})$ containing various outer trehalose or sucrose concentrations. The lipid concentration for all of the samples is $50 \mathrm{mM}$, thus the lowest amount of trehalose or sucrose in any of these systems corresponds to 1:1 ratio. During the freezing step of lyophilization, the membrane (prepared in the liquid crystalline phase) undergoes a phase transition to the gel phase. The tighter packing of the gel phase lipids results in a smaller number of trehalose molecules to interact (via water replacement) with the phospholipids during the drying process. Therefore, Tm is shifted towards high temperature, $83^{\circ} \mathrm{C}$. For the sample containing $50 \mathrm{mM}$ trehalose, the $\mathrm{Tm}$ is observed at approximately $73.5^{\circ} \mathrm{C}$ (Figure 5.5; lower panel), ). The transition is decreased to $67.02^{\circ} \mathrm{C}$ upon increasing the trehalose concentration inside the vesicle to $100 \mathrm{mM}$, while it decreases to approximately $55.62^{\circ} \mathrm{C}$ for the samples containing $400 \mathrm{mM}$ trehalose outside the vesicle. In the fully hydrated state, the decrease in Tm is mainly observed for the sample containing high trehalose concentration inside the vesicle. ${ }^{304}$

Note that the shift in the Tm, observed at the various trehalose distributions, is amplified significantly upon dehydration. The largest Tm change, observed upon the addition of 400 $\mathrm{mM}$ trehalose to liposomes, increases from $1{ }^{\circ} \mathrm{C}$ in the fully hydrated state to $28^{\circ} \mathrm{C}$ in the dried state. Additionally, a second peak, or a shoulder, is observed for the dry sample containing $400 \mathrm{mM}$ trehalose, mirroring the two transitions observed in the hydrated state (before lyophilization; Figure 5.3A). Collectively, our results demonstrate that the 

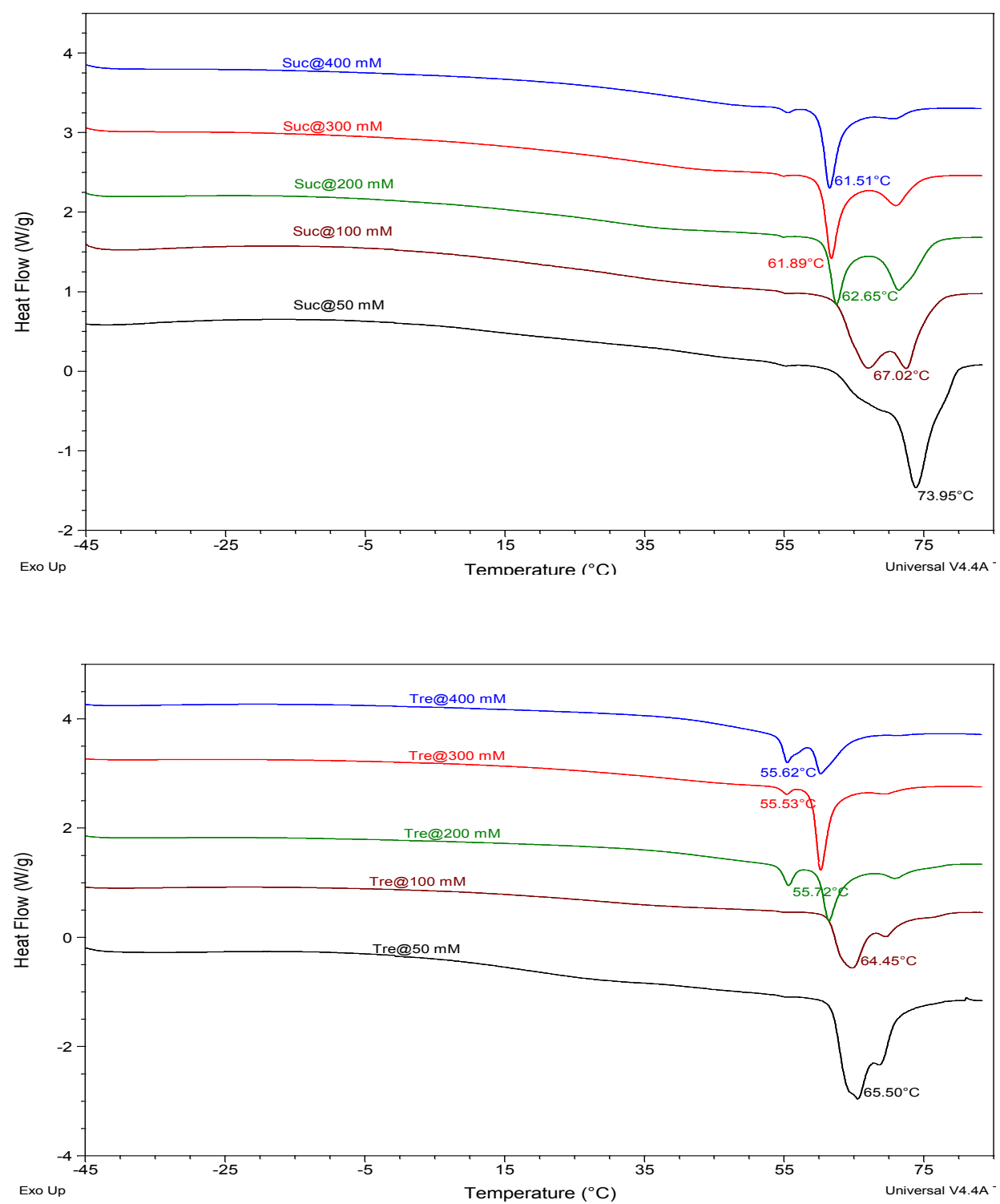

Figure 5.5 Thermograms of lyophilized, rehydrated MPS-L ${ }^{\text {CRGD }}$ formulations containing increasing concentrations of sugar.

The top panel represents that of thermograms with sucrose and the lower panel with trehalose. 
trehalose distribution in the fully hydrated state is maintained upon lyophilization. More importantly, the direct interaction (via hydrogen bonding) between trehalose ( $400 \mathrm{mM}$ trehalose) and phospholipid in the hydrated state is not only maintained, but amplified upon dehydration. Similar pattern was observed with sucrose.

Explanation for the peak at right hand side of the main transition, $52^{\circ} \mathrm{C}$ could be that the lyophilization causes phase separation in the bilayer, resulting in highly ordered HSPC domains, which are difficult to rehydrate. Since the particle size distribution is conserved upon lyophilization and rehydration, the incomplete rehydration cannot be caused by formation of larger aggregates. Further investigations are necessary to verify the reason for the peak observed at $52^{\circ} \mathrm{C}$. The results from this study show that sucrose is needed in higher concentrations than trehalose to protect MPS-L ${ }^{\text {cRGD }}$ during lyophilization and maintain a similar gel-to-liquid phase transition pattern after rehydration. This is in accordance with other studies ${ }^{308}$ and could be explained by a stronger interaction between the lipid membrane and trehalose than with sucrose. ${ }^{303,309}$ Another reason could be the larger hydration volume of trehalose compared to sucrose making it a better kosmotrope. $^{310}$

During freezing and drying this in turn leads to minimized damage by water crystallization. ${ }^{311}$ A final explanation could be the high Tg of trehalose in the dry state compared to other sugars ${ }^{248,312}$ making it a better vitrifier as it is less prone to crystallization than many other sugars. ${ }^{248}$ Crystallization of the sugars could cause a lowered Tg due to residual water, but this would also make the lyophilized cake collapse. Since no collapse was observed in formulations with more than $200 \mathrm{mM}$ sugar, residual water is not believed to cause crystallization. Furthermore, since crystallization of the sugars is a slow process below $\mathrm{Tg}$, there is no reason to believe that trehalose should be better to protect MPS- $\mathrm{L}^{\mathrm{cRGD}}$ during lyophilization, because of its abilities as a vitrifier. It is therefore unlikely that the superior effect of trehalose compared to sucrose is due to its vitrifying effects. In this case, we consider direct interaction with HSPC to be the major reason for the protective effect of sucrose and trehalose. The beneficial effect of trehalose compared to sucrose could on the other hand be due to its quality as a kosmotrope and its strong direct interaction with HSPC.

The data presented here prove that trehalose is able to protect surface modified liposomes. The mechanism of action is not yet fully understood, but these data suggest that the direct interaction with HSPC possibly plays a role, whereas the cosmotropic effect and vitrification of the sugars might be the main causes. Based on these physicochemical data, we have demonstrated that trehalose in concentrations from $200 \mathrm{mM}$ protects MPS- $\mathrm{L}^{\mathrm{cRGD}}$ during lyophilization and enables rapid rehydration (within $10 \mathrm{~min}$ ).

\subsubsection{Amino Acids as Lyoprotectants: Influence of Lyoprotectant Concentration}

To investigate the efficiency of amino acids as lyoprotectants, liposomes were prepared and subjected to lyophilization in the presence of various concentrations of the lyoprotectants ranging from $2-8 \mathrm{~mol} / \mathrm{mol}$ of lipid. Liposomes formulated from 
$\mathrm{HSPC} / \mathrm{CHOL} / \mathrm{DSPE}-\mathrm{PEG}_{2000}(50: 45: 5 \mathrm{~mol})$ were subjected to a freezing cycle at $-45^{\circ} \mathrm{C}$ for $2 \mathrm{~h}$ followed by primary drying at $-36^{\circ} \mathrm{C}$ for $36 \mathrm{~h}$ and, secondary drying at $10^{\circ} \mathrm{C}$ for $24 \mathrm{~h}$. Prior to lyophilization, liposomes were in the range, 100-120 $\mathrm{nm}$. When lyophilized in the absence of lyoprotectants, liposomes of 2-3 $\mu \mathrm{m}$ size were formed after rehydration, an almost 25 times over the original liposomes demonstrating aggregation/fusion of liposomes. Alternatively, formulations lyophilised in the presence of the aminoacids (lysine, histidine or arginine) showed lyoprotection with rehydrated liposomes smaller than the control size $(\sim 2250 \mathrm{~nm})$. Both aminoacid and its molar concentration influenced the lyoprotection efficiency. The lyoprotection profiles of the three amino acids were biphasic in nature, but maximal protection against liposome aggregation varied with the amino acids. At low [lyoprotectant/lipid: 2:1] concentration, lysine, histidine or arginine increased liposome size to 3-4 times over the pre-lyophilized liposomes (Table 5.1). With an increase in lyoprotectant/lipid ratio to 4:1, liposome size retention was observed with all the three amino acids. However, lysine offered better protection with liposome size similar to that of liposomes prior to lyophilization ( $110 \mathrm{~nm})$. Histidine and arginine showed a small increase in size to around $200 \mathrm{~nm}$ compared to pre-lyophilized liposomes. TEM analysis of the rehydrated lysine incorporated lyophilized formulation revealed stable, spherical and uniform sized liposomes (Figure 5.6).

Interestingly, a further increase in amino acid concentration resulted in the decrease of lyoprotection. Liposome lyophilized in the presence of lysine or histidine or arginine $(8: 1)$ demonstrated a significant $(\mathrm{P}<0.05)$ increase in liposome size (Table 5.1). Suzuki et al. ${ }^{313}$ reported the biphasic nature of lyoprotectants. During lyophilization, liposomes if unprotected will undergo membrane damage due to the formation of ice crystals and will ultimately flatten and fuse during dehydration and subsequent rehydration. ${ }^{296}$ As amino acids are charged molecules they are capable of interacting with the phosphate head group of the lipid molecule, which is an important feature exhibited by most of the saccharides. ${ }^{247}$ This offers the potential for the amino acids to form a layer on the liposome surface which could possibly be acting as a protective shield against ice crystal damage. In addition, all the amino acids used has a side chain functional group that reacts with a proton at $\mathrm{pH}$ 6-7 to form a charged ionic group which is capable of forming hydrogen bonds with the phosphate of the lipid head group. Histidine is characterized by the presence of a pentacyclic ring in its structure. Arginine and lysine have the longest side chains with lysine having a terminal amino group and arginine with a terminal guanidinium group. During lyophilization, it is possible that the amino acids interact with the phosphate group and form a barrier around the liposomes with layers of amino acids, possibly acting as cushions against any damage during lyophilization. ${ }^{314}$

Saccharides as lyoprotectants suggested that interactions between the saccharide and the lipid molecules may depend on the hydrophobic/hydrophilic nature of the saccharide molecule, with more hydrophobic moieties promoting membrane fusion at higher concentrations. Therefore, the biphasic nature of the amino acid lyoprotection shown in Table 5.1 may be an outcome of high amino acid concentrations, promoting liposome fusion via either hydrophobic interactions or electrostatic interactions between the positively charged amino acids and the slightly anionic surface of the phospholipid membranes. 
Table 5.1 The effect of lyoprotectant concentration on the mean diameter of empty liposomes (after lyophilization).

\begin{tabular}{lcccc}
\hline & \multicolumn{3}{c}{ Mean liposome diameter (nm) } \\
\cline { 2 - 5 } Lyoprotectant/lipid ratio & Control & Lysine & Histidine & Arginine \\
\hline & $2250 \pm 320$ & NA & NA & NA \\
$2: 1$ & & $489 \pm 45$ & $645 \pm 35$ & $389 \pm 15$ \\
$4: 1$ & & $127 \pm 15$ & $190 \pm 8$ & $184 \pm 14$ \\
$6: 1$ & & $512 \pm 18$ & $644 \pm 18$ & $672 \pm 12$ \\
$8: 1$ & & $985 \pm 47$ & $751 \pm 18$ & $653 \pm 12$ \\
\hline
\end{tabular}

All measurements are a mean \pm SEM, $n=2$ samples. Prior to lyophilization, liposomes were in the 100-120 $\mathrm{nm}$ size range.
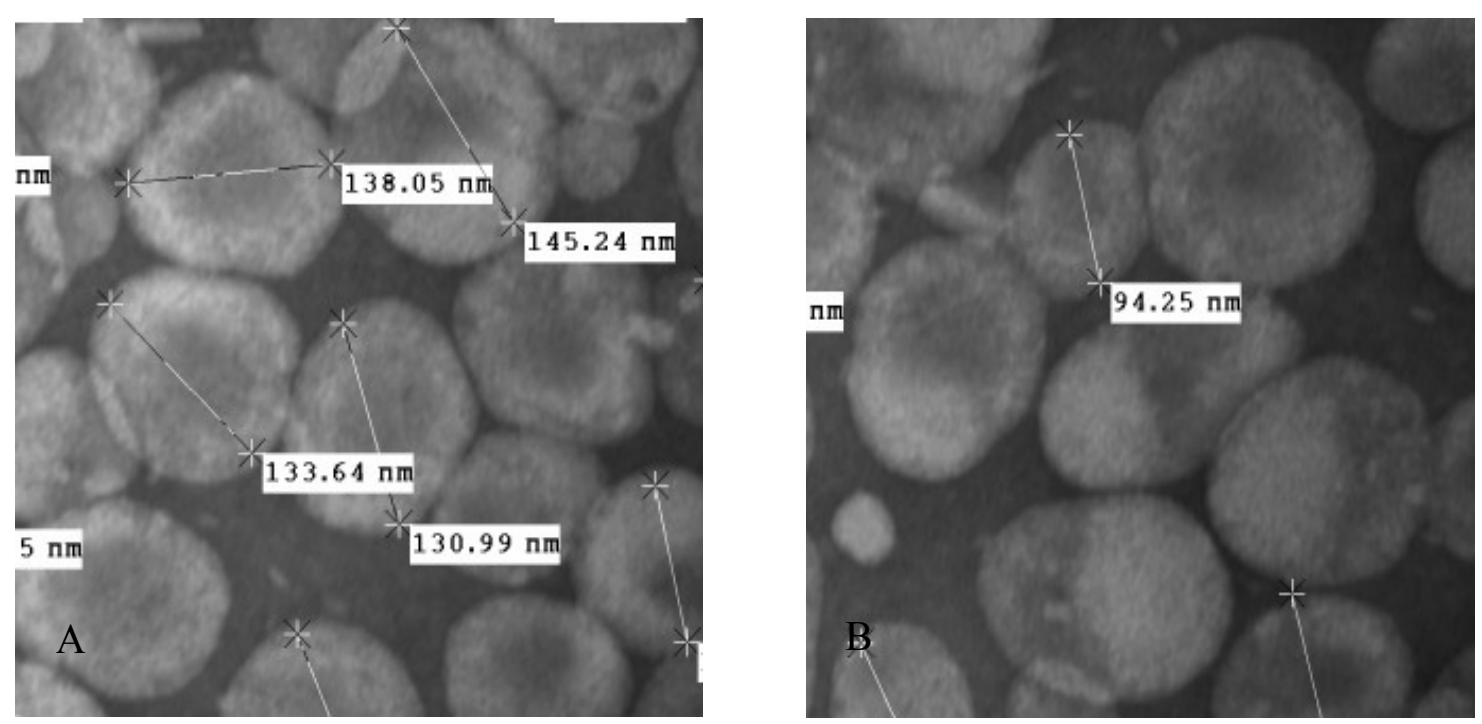

Figure 5.6 Transmission electron micrographs of MPS-L before (A) and after (B) lyophilization with lysine as external lyoprotectant.

Samples were diluted 40 times and subjected to $2 \% \mathrm{w} / \mathrm{v}$ uranyl acetate treatment before acquisition of images $(50,000 \mathrm{x}$ magnification). 


\subsubsection{Influence of Freezing Temperature on the Performance of the Lyoprotectants}

To assess the influence of primary drying temperature on the lyoprotection, liposomes with [lyoprotectant/lipid] ratio at 4:1 were lyophilized. Results (Table 5.2) show that the primary drying temperature influences the liposome product characteristics; formulations subjected to program 1 and 2 were offered protection during lyophilization, with rehydrated liposome size remaining below $200 \mathrm{~nm}$ when any of the aminoacids were employed. In contrast, rehydrated MPS-L subjected to primary drying at $-20^{\circ} \mathrm{C}$ followed by secondary drying at $10^{\circ} \mathrm{C}$ in the presence of lyoprotectant resulted in significant liposome aggregation/fusion, with resultant liposome sizes similar to liposomes lyophilized in the absence of lyoprotection. Crowe et al. ${ }^{247}$ showed that the lyoprotection offered by various solutes is influenced by various factors, including the phase transition temperature of the lipids ( $\mathrm{Tm})$ and the glass transition temperature ( $\mathrm{Tg}^{\prime}$ ) of the amorphous frozen matrix.

In order to preserve the porous nature of the lyophilized cake, primary drying should be performed at approximately $4^{\circ} \mathrm{C}$ below the $\mathrm{Tg}$ ' of the amorphous frozen matrix. For example, trehalose frozen matrix has a $\mathrm{Tg}$ ' of $-32^{\circ} \mathrm{C}$ and when the primary drying temperature was maintained below this temperature (program 1 and 2; Table 5.2), stable products were produced. However, when the frozen samples were subjected to a primary drying temperature around the Tg of the lyoprotectant (program 3; Table 5.2), liposome fusion occurs, possibly due to lack of formation of the glassy state which is responsible for inhibition of liposome aggregation. ${ }^{247}$ Investigation of the influence of duration of primary drying showed that there was no significant difference in the sizes of lyoprotected liposomes when primary drying was carried out at $-36^{\circ} \mathrm{C}$ for 36 or $48 \mathrm{~h}$.

\subsubsection{Influence of Lyoprotectants on MPS Leakage from MPS-L}

Investigations on the protection offered by the amino acids were also extended to determine MPS leakage from MPS-L. In the presence of the three amino acids, MPS-L particle size showed a similar biphasic pattern (Table 5.3) to that of the lyophilized 'empty' liposome shown in Table 5.1. Thus, the presence of MPS within the liposomes did not influence the lyoprotection offered by amino acids suggesting that MPS does not interact with the cushioning effect of amino acids. The moisture levels of all the formulations were less than $3 \% \mathrm{w} / \mathrm{w}$. The presence of low moisture content in the product confers improved stability and drug retention. ${ }^{213}$ This is also in line with the regulations laid down by the regulatory authorities that prefer a maximum water concentration of $3 \%$ w/w.

To determine the particle size distribution pattern and MPS leakage from MPS-L, lyophilized MPS-L were stored at $2-8^{\circ} \mathrm{C}$ and $25^{\circ} \mathrm{C}$ for 4 weeks. With all the three aminoacids as lyoprotectants, a slight increase in liposome size (Figure 5.7) and MPS leakage (Figure 5.8) were observed. Among the three aminoacids, lysine offered protection with a minimal size increase and MPS leakage. It is evident that lyoprotection of liposomes is related to the structure-functional activity of aminoacids. An increase in 
Table 5.2 The effect of lyophilization protocols on the mean diameter of liposomes.

\begin{tabular}{ccccccccc}
\hline Size* & \multicolumn{3}{c}{ Lyophilization protocol temperature $\left({ }^{\circ} \mathrm{C}\right)$} & \multicolumn{3}{c}{ Vesicle diameter after rehydration $(\mathrm{nm})$} \\
\hline \multirow{3}{*}{$110 \pm 13$} & Program & Freezing & Primary Drying & Secondary Drying & Control & Lysine & Histidine & Arginine \\
& 1 & -45 & -36 & 10 & $2564 \pm 124$ & $199 \pm 21$ & $142 \pm 17$ & $157 \pm 8$ \\
& 2 & -45 & -20 & 10 & $3012 \pm 241$ & $145 \pm 14$ & $187 \pm 16$ & $146 \pm 21$ \\
& 3 & -45 & -10 & 10 & $3845 \pm 108$ & $3084 \pm 92$ & $4012 \pm 402$ & $3157 \pm 274$ \\
\hline
\end{tabular}

Size prior to lyophilization (nm). All measurements were a mean \pm SEM, $\mathrm{n}=2$ samples. Prior to lyophilization, liposomes were in the $100-120 \mathrm{~nm}$ size range.

Table 5.3 The effect of lyoprotectant concentration of the mean diameter of MPS-L.

\begin{tabular}{lcccc}
\hline Lyoprotectant/lipid ratio & \multicolumn{3}{c}{ Vesicle diameter (nm) } \\
\hline & Control & Lysine & Histidine & Arginine \\
$2: 1$ & $2850 \pm 124$ & $437 \pm 13$ & $538 \pm 25$ & $389 \pm 15$ \\
$4: 1$ & & $189 \pm 5$ & $176 \pm 13$ & $184 \pm 14$ \\
$6: 1$ & & $486 \pm 91$ & $644 \pm 18$ & $672 \pm 12$ \\
$8: 1$ & & $1356 \pm 91$ & $751 \pm 18$ & $653 \pm 12$ \\
\hline
\end{tabular}

MPS-L (63\% w/v loading efficiency) were prepared and mixed with increasing concentrations of lyoprotectants and lyophilized according to protocol 1 as outlined in Table 5.2. Control liposomes were subjected to the same lyophilization protocol in the absence of lyoprotectants. Mean diameter of liposomes was determined using a zeta sizer. All measurements were a mean \pm SEM, $n=2$ samples. Prior to lyophilization, liposomes were in the 100-120 $\mathrm{nm}$ size range. 


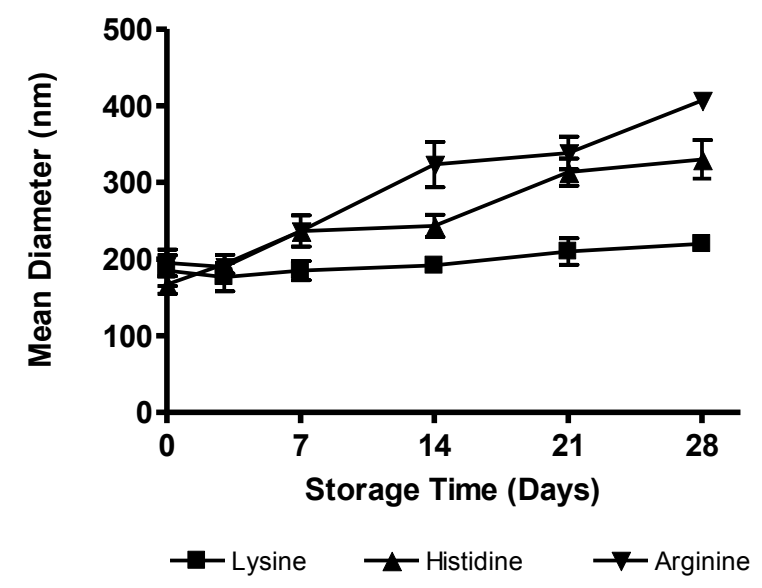

Figure 5.7 Graph depicting the mean diameter of MPS-L as a function of aminoacids as external lyoprotectants used for lyophilization.

Liposomes lyophilized with 4 moles of lyoprotectants/mole of the lipid were stored at $25^{\circ} \mathrm{C}$. At selected time intervals, 1, 3, 7, 14, 21, 28 days, samples were reconstituted and mean diameter of the liposomes was measured. All measurements are a mean \pm SEM, $n=2$ samples.

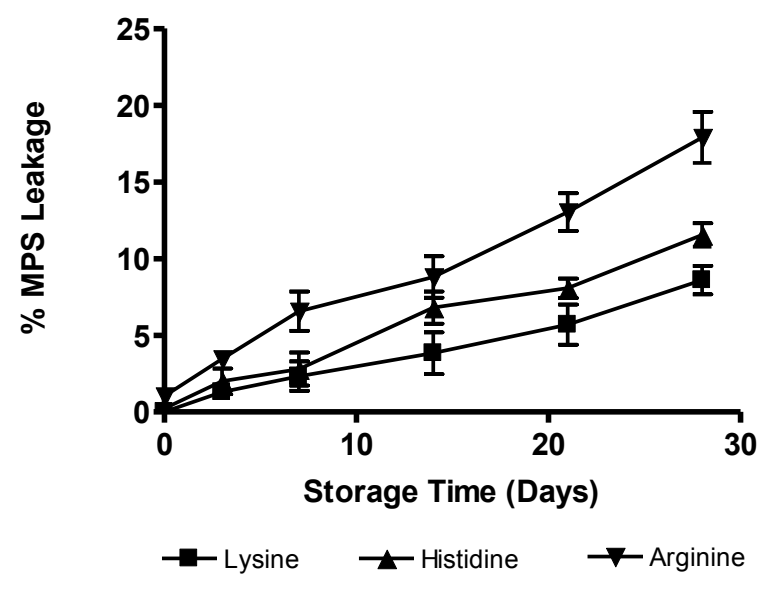

Figure 5.8 Graph depicting the MPS leakage from MPS-L, lyophilized with aminoacids as external lyoprotectants.

Liposomes lyophilized with 4 moles of lyoprotectants/mole of the lipid were stored at $25^{\circ} \mathrm{C}$. At selected time intervals, 1, 3, 7, 14, 21, 28 days, samples were reconstituted and MPS leakage from MPS-L was measured. All measurements are a mean \pm SEM, $n=2$ samples. 
liposome size and MPS leakage over time was observed with arginine or histidine as lyoprotectants. These observations may be attributed to liposome fusion promoted by weak interactions of the amino acids with the phosphate head groups. Therefore, the stability of liposomes was compromised, thus promoting high rates of MPS leakage.The results indicate that lysine appears to offer lyoprotection to liposomes similar to that of trehalose. The ability of amino acids to protect the formulation during lyophilization demonstrates another class of lyoprotectants. 


\section{CHAPTER 6. INHIBITION OF LUNG INFLAMMATION USING MPS-L ${ }^{\text {CRGD }}$}

\subsection{Introduction}

Acute lung injury is a fatal syndrome in critically ill patients with a high mortality rate. ${ }^{315}$ ALI syndrome is characterized by increased permeability of the alveolar-capillary barrier resulting in influx of protein rich edema fluid, and consequently impairment in arterial oxygenation. Low tidal-volume mechanical ventilation, a non-pharmacological approach is the only proven strategy to improve the survival of ALI patients. Despite an improved understanding of the pathogenesis of ALI, effective pharmacological therapies are currently not available. Numerous clinical trials involving pharmacological therapies (e.g. anti-inflammatory agents, anti-coagulants and pulmonary surfactants) aimed at different pathological targets turned unsuccessful. One of the attributed drawbacks is the underappreciation of drug delivery systems in the management of ALI. ${ }^{316}$

During the early ALI phase, numerous pro-inflammatory mediators are released into the alveolar spaces in response to various inflammatory stimuli. ${ }^{4}$ Among them, IL- $1 \beta$ causes pulmonary epithelial and endothelial barrier dysfunction leading to an increase in lung permeability and edema. ${ }^{317-319}$ Ganter et al. reported that IL-1 $\beta$ mediates alveolar epithelial permeability via $\alpha v \beta 6$ integrin dependent TGF- $\beta$ activation. ${ }^{21}$ The $\alpha v \beta 6$ integrin receptors of the alveolar epithelium mediate interaction of the latent TGF- $\beta$ with RGD domain of the LAP. As a result, the latent TGF- $\beta$ undergoes activation leading to an increase in epithelial permeability. ${ }^{19}$ A transient blockade of $\alpha \mathrm{v} \beta 6$ integrin receptors would therefore be a potential strategy to prevent both TGF- $\beta$ activation and an increase in epithelial permeability.

IL-1 $\beta$ stimulates pro-inflammatory cytokine and chemokine release, and upregulates CAMs in alveolar epithelial cells. ${ }^{320}$ Due to a high chemokine gradient across the alveolar barrier, leukocytes (e.g. monocytes and neutrophils) infiltrate from blood circulation into alveoli, and exacerbate edema and lung inflammation. ${ }^{321,322}$ Of the various transcriptional factors, NF- $\mathrm{B}$ p plays a central role in regulating the inflammatory genes encoding CAMs, chemokines and cytokines. ${ }^{75,323} \mathrm{NF}-\kappa \mathrm{B}$ is a heterodimeric protein present in the cytoplasm in an inactive form stabilized by binding to the inhibitory protein, $\mathrm{I} \kappa \mathrm{B} \alpha{ }^{74} \mathrm{In}$ response to IL- $1 \beta$ stimulation, I $\kappa \mathrm{B} \alpha$ undergoes phosphorylation and proteolytic degradation. As a result, phosphorylated $I_{\kappa} B \alpha\left(p-I_{\kappa} B \alpha\right)$ is ubiquitinated allowing nuclear translocation of $\mathrm{NF}-\kappa \mathrm{B}$, which binds to promoter regions of the target genes to initiate the transcription of CAMs, chemokines and pro-inflammatory cytokines. ${ }^{324,325}$ And inhibitors targeting the activated $\mathrm{NF}-\kappa \mathrm{B}$ would suppress CAM expression and chemokines release, which eventually prevent migration of leukocytes. ${ }^{326,327}$ Glucocorticoids, cited as NF- $\mathrm{KB}$ inhibitors mediate the effects through inhibition of $\mathrm{NF}-\kappa \mathrm{B}$ transcriptional activity leading to suppression of pro-inflammatory genes. ${ }^{328}$ Taken together, IL- $1 \beta$ mediates: I) $\alpha v \beta 6$ integrin dependent epithelial permeability and II) induction of NF- $\kappa \mathrm{B}$ dependent proinflammatory genes. In order to alleviate both pulmonary edema and inflammation associated with IL-1 $\beta$ stimulation, I used MPS-L ${ }^{\text {cRGD }}$. 
In the present study, liposomes were chosen for their flexibility: I) to anchor cRGDpeptide onto the surface of liposomes for targeting to $\alpha v \beta 6$ integrin receptors located on alveolar epithelium and II) to encapsulate and deliver MPS, an anti-inflammatory glucocorticoid to alveolar epithelial cells for inhibition of pro-inflammatory cytokines (e.g. TNF- $\alpha$ and IL-6), chemokines (e.g. MCP-1) and CAMs (e.g. ICAM-1) expression.

To evaluate the potential of MPS-L ${ }^{\mathrm{cRGD}}$ treatment in modulating epithelial permeability, we examined IL-1 $\beta$ stimulated A549 cells. A significant decrease in albumin flux across A549 cells was observed. In addition, MPS-L ${ }^{\text {cRGD }}$ targeting the $\alpha v \beta 6$ integrin receptors undergo cell internalization and suppress MCP-1 and ICAM-1 expression. To determine whether such an effect might have relevance to disease, we used IL-1 $\beta$ induced lung inflammation rat model. MPS-L ${ }^{\text {CRGD }}$ suspension was nebulized at a rate of $1 \mathrm{~mL} / \mathrm{min}$ for 5 min prior to instillation (i.t.) of IL- $1 \beta$ in male Sprague-Dawley rats. In vivo results demonstrated that MPS-L ${ }^{\text {cRGD }}$ treatment show a decrease in epithelial permeability, neutrophils migration and pro-inflammatory mediators' expression compared to IL-1 $\beta$ instilled (i.t.) positive control. The results of this study present evidence that MPS-L ${ }^{\text {cRGD }}$ reduces pulmonary edema by blocking $\alpha v \beta 6$ integrin receptors; while MPS release from MPS-L ${ }^{\text {cRGD }}$ inhibits NF- $\kappa$ B transcriptional activity, at least in part by increasing $\mathrm{I} \kappa \mathrm{B} \alpha$ leading to suppression of MCP-1 and ICAM-1 production.

\subsection{Experimental Section}

\subsubsection{Materials}

HSPC1, DSPE-PEG 2000, DSPE-PEG $_{2000}$-maleimide and 1-Oleoyl-2-[6-[(7-nitro-2-1,3benzoxadiazol-4-yl)amino]hexanoyl]-sn-Glycero-3-Phosphoethanolamine (NBD-PE) were obtained from Avanti Polar Lipids (Birmingham, AL). MPS and recombinant human interleukin-1 $\beta$ were purchased from Sigma (St.Louis, MO). cRGD-peptide was purchased from Peptide International Inc. (Louisville, KY). DMEM medium, PBS buffer, HEPES buffer, antibiotics and growth factors were purchased from Cellgro Inc. (Herndon, VA). RPMI 1640 medium was purchased from Gibco (Carlsbad, CA). Antibodies obtained include: Anti- $\alpha$ v (P2W7; sc-9969; Santa Cruz Biotechnology Inc., Santa Cruz, CA), anti- $\beta 6$ (H-110; sc-15329; Santa Cruz Biotechnology Inc), anti- $\beta 1$ (mAb13, Becton-Dickinson, Bedford, MA), anti- $\alpha \mathrm{v} \beta 3$ (LM609; Chemicon, Temecula, CA), anti- $\alpha v \beta 5$ (P1F6; Chemicon) and IgG (Chemicon). Gel electrophoresis chemicals were obtained from Bio-Rad Inc. (Hercules, CA). All other chemicals of ACS grade or higher were purchased either from Sigma (St.Louis, MO) or Fisher Scientific (Suwannee, GA). 


\subsubsection{Methods}

\subsubsection{Preparation of Liposomes}

MPS encapsulation into liposomes was achieved by transmembrane calcium acetate gradient method. Liposomes were prepared with a lipid composition of HSPC, CHOL, DSPE-PEG $_{2000}$ and DSPE-PEG 2000 -maleimide in 50: 45: 3: 2 molar ratios. Lipid (27.2 $\mathrm{mM}$ total phospholipids) solution in chloroform-methanol solution $(3: 1 \% \mathrm{v} / \mathrm{v}) \mathrm{was}$ subjected to vacuum drying at $40^{\circ} \mathrm{C}$ to form a thin dry film, which was hydrated with calcium acetate buffer $(200 \mathrm{mM})$ at $65^{\circ} \mathrm{C}$ to form MLVs. These vesicles were extruded through $200 / 100 \mathrm{~nm}$ sandwiched polycarbonate membrane filters using LIPEX $^{\mathrm{TM}}$ extruder. The MLVs were subjected to 6 extrusion passes to obtain liposomes with a mean diameter of 100-120 nm. For flow cytometry studies, lipid solution was spiked with $3 \mathrm{~mole} \%$ NBD-PE $(\lambda$ ex $=488 \mathrm{~nm}, \lambda$ em $=520 \mathrm{~nm})$ dye during hydration.

To create a transmembrane calcium acetate gradient across the liposomes, the extraliposomal calcium acetate buffer was replaced with $0.9 \%$ saline solution by dialysis (10,000 Da MWCO). Followed by generation of calcium acetate gradient, liposomes were mixed and incubated with MPS solution [19 $\mathrm{mM}$ ] at $48^{\circ} \mathrm{C}$ for $6 \mathrm{~min}$ and then cooled to $4^{\circ} \mathrm{C}$. Unencapsulated MPS and any traces of calcium acetate were removed by dialysis of MPS-L at $4^{\circ} \mathrm{C}$.

To prepare cRGD peptide conjugated liposomes, MPS-L were incubated with cRGD peptide solution $(667 \mu \mathrm{g} / 30 \mu \mathrm{L} ; \mathrm{pH} 6.5)$ at $25^{\circ} \mathrm{C}$ for $12 \mathrm{~h}$. The conjugation reaction was quenched with EDC solution. Unconjugated cRGD peptide and excess EDC were isolated by dialysis using equilibrium chambers separated by a $1000 \mathrm{Da} \mathrm{MWCO}$ membrane (Harvard Apparatus, Holliston, MA). The amount of cRGD peptide conjugated to MPS-L was estimated by subtraction of free cRGD peptide of the dialysate from the total $\mathrm{cRGD}$ peptide used for conjugation. The free cRGD peptide fraction was determined by RP-HPLC method. After dialysis, liposomal MPS and total phospholipids concentrations were $12.02 \mathrm{mM}$ and $23.8 \mathrm{mM}$, respectively. Approximately, 34\% (molar) cRGD peptide was conjugated to the maleimide group of MPS-L.

Mean diameter and zeta potential of liposomes were evaluated by DLS studies as described elsewhere.

\subsubsection{Cell Cultures}

A549 cells from the American Type Culture Collection (Manassas, VA) were grown in Dulbecco's modified Eagle's medium (DMEM) supplemented with 10\% v/v heatinactivated FBS, antibiotics (10 $\mu \mathrm{g} / \mathrm{mL}$ gentamicin and $0.25 \mu \mathrm{g} / \mathrm{mL}$ amphotericin), 1 $\mu \mathrm{g} / \mathrm{mL}$ hydrocortisone, $10 \mu \mathrm{g} / \mathrm{mL}$ human epidermal growth factor, $3 \mathrm{ng} / \mathrm{mL}$ fibroblast growth factor and $10 \mu \mathrm{g} / \mathrm{mL}$ heparin. The cell cultures were incubated in $5 \% \mathrm{CO}_{2}$ at $37^{\circ} \mathrm{C}$. Cells were cultured in T-75 flasks (Beckton Dickinson, Franklin Lakes, NJ) before 
transferring to plates for specific assays. For RT-PCR assays, cells were cultured in collagen-I coated $60 \mathrm{~mm}$ petri dishes (Beckton Dickinson) at a seeding density, $2 \times 10^{6}$ cells per mL. For cell based ELISA, cells were seeded at $2 \times 10^{4}$ per well in collagen-I coated 96 well plates (Becton Dickinson). For monocytes or neutrophils adhesion assays, cells were passed from T-75 flasks and cultured in collagen-I coated 24 well Transwell ${ }^{\circledR}$ filters ( $8 \mu \mathrm{M}$ pore membrane; Becton Dickinson) at a density, $5 \times 10^{4}$ per well. For pemeability assays, cells were cultured in collagen-I coated Transwell $6.5 \mathrm{~mm}$ filters (Costar, Cambridge, MA). Cells were then allowed to quiesce in DMEM incomplete medium overnight before subjecting to treatments. Cells between the passages, 2-4 were used for all studies.

\subsubsection{AlphaVBeta6 (ovß6) Integrin Expression in A549 Cells}

A549 cells were treated with IL-1 $\beta$ (10 ng/mL; $3 \mathrm{~mL}$ total volume) and DMEM incomplete medium (control; $3 \mathrm{~mL}$ ) for $0,3,6$ and $12 \mathrm{~h}$ at $37^{\circ} \mathrm{C}$. Following treatments, cell monolayers were washed with PBS, fixed at $4^{\circ} \mathrm{C}$ for $30 \mathrm{~min}$ in $2 \% \mathrm{v} / \mathrm{v}$ paraformaldehyde and washed again with PBS. Non-fat dry milk $(5 \% \mathrm{w} / \mathrm{v} ; 300 \mu \mathrm{L})$ was added to reduce non-specific binding. Cells were then incubated with primary monoclonal antibodies $(100 \mu \mathrm{L} ; 100 \mu \mathrm{g} / \mathrm{mL}$ in $1 \% \mathrm{w} / \mathrm{v}$ BSA-PBS solution) for $1 \mathrm{~h}$. The monolayers were washed with PBS-Tween20 $(0.01 \% \mathrm{w} / \mathrm{v}$; PBST) buffer and incubated with biotinylated anti-mouse secondary antibody $(100 \mu \mathrm{L}$ of $1 \mu \mathrm{g} / \mathrm{mL})$ for $1 \mathrm{~h}$. Subsequently, cells were washed as above and incubated with streptavidin horseradish peroxidase $(100 \mu \mathrm{L}$; DAKO, Carpinteria, CA) for $30 \mathrm{~min}$. After washing with PBST buffer, TMB peroxidase substrate (Sigma, MO) was added and incubated for $30 \mathrm{~min}$. The reaction was halted by addition of $2 \mathrm{~N}$ sulfuric acid $(50 \mu \mathrm{L} /$ well $)$. All incubations were carried out at room temperature. The expression level of $\alpha v \beta 6$ integrin receptors was determined by difference in absorbance at wavelengths $450 \mathrm{vs} .630 \mathrm{~nm}$. An isotype matched control $\mathrm{Ab}(\mathrm{IgG})$ was used in place of primary antibody in three wells and background was subtracted from the signal.

RT-PCR was performed to estimate mRNA expression of $\alpha \mathrm{v} \beta 6$ integrin receptors. Cells were grown and subjected to treatments as described elsewhere. Cells were lysed with TRIzol ${ }^{\circledR}$ reagent and total cell RNA was extracted according to manufacturer's instructions (Invitrogen, Carlsbad, CA). Equal aliquots (4 $\mu \mathrm{g})$ of RNA were quantified at $260 \mathrm{~nm}$ absorbance. RNA was reverse transcribed into cDNA synthesis with an oligo (dT) primer using the Superscript III First-Strand Synthesis System as described in the manufacturer's instructions. Primers were designed using Primer3 software (http://frodo.wi.mit.edu). The primers were purchased from Integrated DNA technologies (Coralville, IA). Primer sequences are listed in Table.6.1. The concentration of primers was $200 \mathrm{nM}$ for forward and reverse primers. PCR amplification conditions were: $50^{\circ} \mathrm{C}$ for $30 \mathrm{~min}, 1$ cycle; $94^{\circ} \mathrm{C}$ for $5 \mathrm{~min}, 1$ cycle; 35 cycles of $94^{\circ} \mathrm{C}$ for $45 \mathrm{~s}, 55-58^{\circ} \mathrm{C}$ for $45 \mathrm{~s}$ and $72^{\circ} \mathrm{C}$ for $2 \mathrm{~min} ; 68^{\circ} \mathrm{C}$ for $7 \mathrm{~min}, 1$ cycle. Ten microliters of PCR amplification product and a 123-bp DNA ladder were separated using agarose $(1.5 \% \mathrm{w} / \mathrm{v})$ gel electrophoresis. Gels were stained with ethidium bromide and photographed under ultraviolet transillumination. 
Table 6.1 Primer sequences for $\alpha v, \beta 6$, MCP-1, ICAM-1 and $\beta$-actin.

\begin{tabular}{|c|c|c|c|c|}
\hline Primer & Primer sequence $\left(5^{\prime} \rightarrow 3^{\prime}\right)$ & Product size (bp) & $\mathrm{Tm}$ & $\begin{array}{c}\text { Genbank access } \\
\text { No. }\end{array}$ \\
\hline$\alpha \mathrm{V}$-integrin forward primer & 5'-TATGCTACATCTTGACCCACTA-3' & 104 & 55.0 & NM_002210 \\
\hline$\alpha \mathrm{V}$-integrin reverse primer & 5'-CCAATTAGCACTATCAGCAGTA-3' & & & \\
\hline$\beta 6$-integrin forward primer & 5'-TGAATACTCCATGTCAACTGTC-3' & 150 & 55.14 & NM_000888 \\
\hline$\beta 6$-integrin reverse primer & 5'-GTAGCTCCAGGAATAAGTTTTG-3' & & & \\
\hline MCP-1 forward primer & 5'-AАСТTATTTTCСССТАGCTTTC-3' & 172 & 55.26 & NM_002982 \\
\hline MCP-1 reverse primer & 5'-СССCAAGTCTCTGTATCTAAAA-3' & & & \\
\hline ICAM-1 forward primer & 5'-TCAGTCAGATACAACAGCATTT-3' & 223 & 55.07 & NM_000201 \\
\hline ICAM-1 reverse primer & 5'-ATAGGCAGCAAGTTTCAGTATT-3' & & & \\
\hline$\beta$-actin forward primer & 5'-CATTGCTTTCGTGTAAATTATG-3' & 213 & 55.0 & NM_001101 \\
\hline$\beta$-actin reverse primer & 5'-TTATTCAACTGGTCTCAAGTCA-3' & & & \\
\hline
\end{tabular}




\subsubsection{Confocal Laser Scanning Microscopy (CLSM)}

MPS-L ${ }^{\text {CRGD }}$ affinity to $\alpha v \beta 6$ integrin receptors of A549 cells was determined by fluorescence microscopy using NBD-PE labeled, specific MPS- $\mathrm{L}^{\mathrm{cRGD}}$ and non-specific liposomes (MPS-L). A549 cells were treated with either IL-1 $\beta$ or DMEM complete medium (control) for $6 \mathrm{~h}$ at $37^{\circ} \mathrm{C}$. Followed by IL-1 $\beta$ treatment, A549 cells were treated with anti- $\alpha v \beta 3$, anti- $\alpha v \beta 5$ and anti- $\beta 1$ integrin antibodies $(10 \mu \mathrm{g} / \mathrm{mL})$ to facilitate a high degree of binding of MPS- $\mathrm{L}^{\mathrm{CRGD}}$ to $\alpha \mathrm{v} \beta 6$ integrins. After washing with DMEM incomplete medium, cells were incubated separately with the NBD-PE labeled liposome formulations $\left(50 \mu \mathrm{M}\right.$ phospholipids $/ 10^{6}$ cells) at $37^{\circ} \mathrm{C}$ for $1 \mathrm{~h}$. The total volume of liposome formulation was $100 \mu \mathrm{L}$ containing the following (in $\mathrm{mM}$ ): $\mathrm{NaCl}$ (115), $\mathrm{KCl}$ (5), $\mathrm{NaHCO}_{3}(10)$, $\mathrm{HEPES}(25), \mathrm{MgCl}_{2}(0.5), \mathrm{CaCl}_{2}$ (1), glucose (5.6) and BSA (1 $\mathrm{mg} / \mathrm{mL}$ ). Unbound liposomes were removed by washing the plates with ice-cold PBS buffer. Followed by treatments, the cells were fixed in paraformaldehyde and subjected to microscopy under 100,000x maginification.

\subsubsection{MCP-1 and ICAM-1 Expression in A549 Cells}

A549 cells were treated with IL-1 $\beta(10 \mathrm{ng} / \mathrm{mL} ; 3 \mathrm{~mL}$ total volume $)$ and DMEM incomplete medium (control; $3 \mathrm{~mL}$ ) for $0,3,6$ and $12 \mathrm{~h}$ at $37^{\circ} \mathrm{C}$. In pretreatment protocol, A549 cells were pretreated with MPS-L or MPS-L ${ }^{\text {CRGD }}$ (equivalent to 0.03-30 $\mu \mathrm{M}$ MPS) or $\mathrm{L}^{\mathrm{CRGD}}$ for $1 \mathrm{~h}$ followed by IL-1 $\beta(10 \mathrm{ng} / \mathrm{mL})$ incubation for $6 \mathrm{~h}$. Cells were analyzed for MCP-1 and ICAM-1 levels by Western Blot and RT-PCR procedures which were described elsewhere. Primer sequences and RT-PCR conditions are listed in Table 6.1.

\subsubsection{Isolation of Monocytes and Neutrophils}

Blood collected from four healthy volunteers was subjected to dextran (Sigma, St.Louis, MO) sedimentation to obtain leukocyte rich plasma, which was layered onto $2.5 \mathrm{~mL}$ of Nycoprep, 1.068 (Invitrogen, Carlsbad, CA) and centrifuged (500xg, $20 \mathrm{~min}, 20^{\circ} \mathrm{C}$ ). The plasma and cells were then collected into separate tubes and centrifuged $(600 \mathrm{xg}, 10 \mathrm{~min}$, $20^{\circ} \mathrm{C}$ ). The monocyte pellet was then resuspended in RPMI 1640 incomplete medium and centrifuged $\left(90 \mathrm{xg}, 15 \mathrm{~min}, 20^{\circ} \mathrm{C}\right)$ again to remove platelets. This was repeated six times. The resulting cells were resuspended in RPMI 1640 containing $1 \% \mathrm{w} / \mathrm{v} \mathrm{PSG}, 0.5 \% \mathrm{w} / \mathrm{v}$ BSA and $10 \% \mathrm{v} / \mathrm{v}$ FCS (Cellgro) at $1 \times 10^{6}$ per mL. Following this, cells were $90 \%$ pure. For isolation of neutrophils, the leukocyte rich plasma was centrifuged, (400xg, $10 \mathrm{~min}$, $4{ }^{\circ} \mathrm{C}$ ) and the supernatant was discarded. The cell pellet was resuspended in PBS and centrifuged for a second time as before. Following washing, the cell pellet was subjected to discontinuous Percoll gradient (Sigma). Using a discontinuous gradient consisting of three layers $(81,70$ and $55 \% \mathrm{v} / \mathrm{v})$; the cell pellet was resuspended in $3 \mathrm{~mL}$ of $55 \% \mathrm{v} / \mathrm{v}$ (the top layer), which was then overlaid onto the prepared gradient. The cells were then centrifuged $\left(750 \mathrm{xg}, 20 \mathrm{~min}, 4^{\circ} \mathrm{C}\right.$ ) and neutrophils were harvested from the $70 \% / 81 \%$ interface. The neutrophils were washed further in PBS for use in migration assays. 


\subsubsection{Monocytes and Neutrophils Adhesion Assays}

Isolated monocytes or neutrophils were suspended in RPMI 1640 (containing 1\% w/v PSG, $0.5 \% \mathrm{w} / \mathrm{v}$ BSA and $10 \mathrm{mM}$ HEPES, $\mathrm{pH}$ 7.2) medium and radiolabeled by incubating the cells $\left(1 \times 10^{6}\right.$ per $\mathrm{mL}$ ) with $0.05 \mu \mathrm{Ci} \mathrm{NaCrO}_{4}$ (Perkin Elmer, Waltham, MA) at $37^{\circ} \mathrm{C}$ for $1 \mathrm{~h}$. Radiolabeled monocytes or neutrophils were washed twice $(250 \mathrm{~g} ; 5 \mathrm{~min}$, $20^{\circ} \mathrm{C}$ ) with ice-cold incomplete medium to eliminate excess of unincorporated radioactivity and resuspended in RPMI 1640 incomplete medium, respectively. Twenty min later, monocytes or neutrophils were washed and adjusted to a final concentration of $2 \times 10^{6}$ per $\mathrm{mL}$ in RPMI 1640 incomplete medium, supplemented with $\mathrm{CaCl}_{2}(1.3 \mathrm{mM})$ and $\mathrm{MgCl}_{2}(1 \mathrm{mM})$. Prior to addition of radioactive monocytes or neutrophils, A549 cells were exposed to IL-1 $\beta(10 \mathrm{ng} / \mathrm{mL})$ or incomplete medium (control) for $6 \mathrm{~h}$. In a pretreatment protocol, A549 cells were exposed to MPS- ${ }^{\text {cRGD }}$ or MPS-L (equivalent to 0.03-30 $\mu \mathrm{M}$ MPS) or $\mathrm{L}^{\mathrm{cRGD}}$ for $12 \mathrm{~h}$ followed by IL-1 $\beta(10 \mathrm{ng} / \mathrm{mL})$ exposure for $6 \mathrm{~h}$. Radiolabeled monocytes or neutrophils $\left(5 \times 10^{5} /\right.$ well) were then added to treated A549 cells (monocytes or neutrophils /A549 cells: 10/1). After co-incubation at $37^{\circ} \mathrm{C}$ for 30 min, the percentage of monocytes or neutrophils adhered to A549 cells were quantified. Non-adherent monocytes or neutrophils were removed by washing with $0.5 \% \mathrm{w} / \mathrm{v}$ BSAPBS solution. Adherent cells were lysed and counted in a gamma counter (Perkin Elmer, Waltham, MA).

\subsubsection{Epithelial Permeability Assay}

Cells were cultured as described elsewhere. A549 monolayers were grown to confluence until TEER reaches $30 \Omega . \mathrm{cm}^{2}$ (EVOM; World Precision Instruments, Sarasota, FL). Briefly, monolayers were washed in PBS and equilibrated at $37^{\circ} \mathrm{C}$ for $30 \mathrm{~min}$. A549 cells were exposed to IL-1 $\beta(10 \mathrm{ng} / \mathrm{mL})$ or DMEM incomplete medium (control) for $6 \mathrm{~h}$. In a pretreatment protocol, cells were incubated with MPS-L ${ }^{\text {cRGD }}$ or MPS-L (equivalent to 30 $\mu \mathrm{M}$ MPS) or $\mathrm{L}^{\mathrm{cRGD}}$ or antibodies $(10 \mu \mathrm{g} / \mathrm{mL})$ and then stimulated with IL-1 $\beta(10 \mathrm{ng} / \mathrm{mL})$ for $1 \mathrm{~h} .{ }^{14} \mathrm{C}$-albumin $(0.05 \mu \mathrm{Ci})$ (Perkin Elmer, Waltham, MA) was added to upper compartment at $37^{\circ} \mathrm{C}$ for $1 \mathrm{~h}$, after which contents from the lower compartment were collected and counted in scintillation counter.

\subsubsection{Animals}

Male Sprague-Dawley rats, weighing 150-200 g, were purchased from Harlan laboratories and used in all experiments. The protocols were approved by the Institution Animal Care and Use Committee (IACUC) of the University of Tennessee, Memphis.

\subsubsection{Nebulization of Liposome Formulations}

At least $30 \mathrm{~min}$ prior to IL-1 $\beta$ (i.t.) instillation, rats were nebulized with liposome (PL or MPS-L or MPS- $\mathrm{L}^{\mathrm{cRGD}}$ ) formulations using micropump nebulizer (Aeroneb ${ }^{\circledR}$, Aerogen, 
Ireland). Nebulization chamber filled with formulations was placed on the snout of rats. Nebulization was actuated at a rate, $1 \mathrm{~mL} / \mathrm{min}$ for $3 \mathrm{~min}$. MPS-L or MPS-L ${ }^{\text {cRGD }}$ formulations contain MPS dose, $6.3 \mathrm{mg} / \mathrm{mL}$. (Estimation of MPS in Blood and BALF samples was done by RP-HPLC method).

\subsubsection{IL-1 $\beta$ Instillation and BALF Collection}

Male Sprague-Dawley rats, weighing 125-150 g were used in the study. Rats were fed normal diet before the experiment with free access to water. Anesthesia was induced by intra-peritoneal injection of ketamine/xylazine $(87 / 13 \mathrm{mg} / \mathrm{kg})$. Following anesthesia, a midline tracheal incision was made to expose the trachea. A 26 gauge needle was advanced endotracheally to instill IL- $1 \beta$ solution $(200 \mu \mathrm{L}, 100 \mu \mathrm{g} / \mathrm{mL}$ in PBS, pH 7.2) or PBS alone (control). Following the IL-1 $\beta$ instillation, tracheal opening was sutured and animals were returned to cage. Within $15 \mathrm{~min}$ of IL-1 $\beta$ instillation, anti- $\beta 1$ or anti- $\alpha \mathrm{v} \beta 3$ or anti- $\alpha \mathrm{v} \beta 5$ antibodies $(1 \mathrm{mg} / \mathrm{mL} / \mathrm{kg})$ or a combination were administered intravenously (i.v.). Twenty four hours post IL-1 $\beta$ administration, the lung vascular bed was flushed twice by injecting $10 \mathrm{~mL}$ ice-cold PBS into right ventricle. Followed by overdose of pentobarbital $(100 \mathrm{mg} / \mathrm{kg}$, i.v), tracheal sutures were cut open to expose trachea. BALF collection was performed by inserting a 26 gauge needle into trachea and lungs were lavaged twice with PBS ( $3 \mathrm{~mL}$ ). BALF was collected into cryovials and centrifuged at $1100 \mathrm{xg}, 4^{\circ} \mathrm{C}$ for $10 \mathrm{~min}$. The supernatant was stored at $-70^{\circ} \mathrm{C}$ for cytokine and chemokine analysis and the cell pellet was subjected to neutrophils estimation. Aliquots of BALF were estimated for protein concentration by UV absorbance at $280 \mathrm{~nm}$ using albumin (Bio-Rad) as standard.

\subsubsection{Estimation of BALF Cytokines, Chemokines and Adhesion Molecules}

BALF supernatant collected and stored as described above was analyzed for cytokines (TNF- $\alpha$ ), MCP-1 and ICAM-1 levels by ELISA method according to manufacturer (R\&D Systems, Minneapolis, MN) instructions.

\subsubsection{Estimation of Lung Neutrophil Content}

Neutrophil content was assayed as described previously. ${ }^{329}$ Lung tissue from each lobe was pooled $(500 \mathrm{mg})$ and freeze-dried. The samples were then homogenized in HEPES (50 mM, pH 8.0) at $0.5 \% \mathrm{w} / \mathrm{v}$ with tissue homogenizer (Fisher Scientific, Suwannee, GA). Homogenized samples were then centrifuged at $12,000 \mathrm{xg}$ at $4{ }^{\circ} \mathrm{C}$ for $30 \mathrm{~min}$ and the supernatant was discarded. The process was repeated twice. The resulting pellet was homogenized in $0.5 \% \mathrm{w} / \mathrm{v}$ CTAC solution and clarified by repeat centrifugation. The resultant clear supernatant was analyzed for MPO activity. The same protocol was used to extract MPO from BALF cell pellets. The collected supernatant from HEPES/CTAC extracts of lung tissue was diluted 10 fold with $10 \mathrm{mM}$ citrate buffer $(\mathrm{pH} \mathrm{5.0)}$ ) and $100 \mu \mathrm{L}$

of this solution was pipetted into 4 wells of a 96-well plate. As negative control, "stop 
solution" $\left(4 \mathrm{~N} \mathrm{H}_{2} \mathrm{SO}_{4}\right)$ was added to two of the wells. Then, $100 \mu \mathrm{L}$ of the substrate solution (3 mM 3,5,5-tetramethylbenzidine dihydrochloride, $120 \mathrm{mM}$ resorcinol and 2.2 $\mathrm{mM}$ hydrogen peroxide in distilled water) was added to all the wells and the reaction was quenched with stop solution at $25^{\circ} \mathrm{C}$ after 2 min.

A standard curve of MPO activity vs. neutrophils count was developed by homogenizing a known number of Percoll-purified neutrophils using the same technique used in MPO extraction from lung and BALF cells. Lung and BALF neutrophil count was estimated from the standard curve.

\subsubsection{Histological Examination of Lungs}

For histological examination ( $\mathrm{n}=3$ each group), bronchial lavage was omitted and after vascular perfusion, $10 \mathrm{~mL}$ buffered formalin $(3.7 \% \mathrm{v} / \mathrm{v})$ was infused via the pulmonary artery to fix the lung. Lungs were isolated en bloc after tracheal ligation and preserved in $10 \% \mathrm{v} / \mathrm{v}$ neutral buffered formalin for $24 \mathrm{~h}$ at $4^{\circ} \mathrm{C}$. Wedge segments from each lobe were paraffin embedded and $5 \mathrm{~mm}$ sections were taken and stained with haematoxylin and eosin using standard protocol.

\subsubsection{Statistical Analysis}

All data reported are arithmetic means. Error bars represent one SEM. Differences between means of indicated groups were analyzed by ANOVA with Bonferroni corrections for multiple comparisons. A P value $<0.05$ was considered to be statistically significant.

\subsection{Results and Discussion}

The colloidal properties of MPS-L ${ }^{\text {cRGD }}$ and MPS-L determined by DLS method were stable over 14 days of shelf life. As shown in Table 6.2, mean diameter of MPS-L ${ }^{\text {cRGD }}$ and MPS-L were comparable; however, the zeta potential of MPS-L ${ }^{\text {cRGD }}$ was slightly higher than MPS-L due to a partial negative charge of the targeting ligand, cRGDpeptide.

\subsubsection{AlphaVBeta6 ( $\alpha$ v $\beta 6$ ) Integrin Receptor Expression in A549 Cells}

The $\alpha v \beta 6$ integrin receptors are normally expressed at a low level in lung epithelium, but can be upregulated by injury and inflammation. To determine whether IL-1 $\beta$ treatment induces $\alpha v \beta 6$ integrin up-regulation in A549 cells, we performed cell based Western Blot analysis was performed on A549 cells after treatment with either IL-1 $\beta$ or saline. A549 cells were stimulated with IL- $1 \beta$ for various periods of time. After $3 \mathrm{~h}, \beta 6$ subunit protein expression increased by 1.8 folds $(\mathrm{P}<0.05)$ and after $6 \mathrm{~h}$ reached a maximum of 2.9 folds 
Table 6.2 Physico-chemical properties of MPS- $\mathrm{L}^{\mathrm{cRGD}}$ used in in vitro and in vivo studies.

\begin{tabular}{lccc}
\hline Liposomes & $\begin{array}{c}\text { Mean diameter, } \\
\text { Zavg }(\mathrm{nm})\end{array}$ & Zeta potential, $\zeta(\mathrm{mV})$ & $\begin{array}{c}\text { MPS incorporation } \\
\text { efficiency }(\% \mathrm{w} / \mathrm{v})\end{array}$ \\
\hline PL & $98 \pm 9$ & $-22.7 \pm 4.3$ & NA \\
PL $^{\text {cRGD }}$ & $110 \pm 6$ & $-17.1 \pm 3.8$ & NA \\
PL $^{\text {cRAD }}$ & $106 \pm 4$ & $-20.8 \pm 6.1$ & NA \\
MPS-L & $113 \pm 5$ & $-22.4 \pm 3.7$ & $\cong 61$ \\
MPS-L & $117 \pm 8$ & $-25.6 \pm 2.2$ & $\cong 36$ \\
\hline
\end{tabular}

Each value represents the mean $\pm \operatorname{SEM}(\mathrm{n}=3)$. NA indicates not applied. NA was applied to the liposome formulations devoid of MPS encapsulation. PL indicates plain liposomes and $\mathrm{PL}^{\mathrm{CRAD}}$ indicates liposomes with RAD as the control targeting ligand, which has no specificity for integrin receptors. 
$(\mathrm{P}<0.05)$ which persisted thereafter (Figure 6.1). The $\alpha \mathrm{v}$ integrin subunit also showed the same pattern. To determine whether $\alpha \mathrm{v} \beta 6$ integrin receptor protein levels reflected mRNA expression levels in IL-1 $\beta$ activated A549 cells, we performed RT-PCR analysis of the cell extracts. Figure 6.1 shows a representative agarose gel demonstrating $\alpha v \beta 6$ integrin expression after stimulation with IL- $1 \beta$ for $0,3,6$ and $12 \mathrm{~h}$. Stimulation with IL$1 \beta$ revealed an up-regulation of $\alpha \mathrm{v} \beta 6 \mathrm{mRNA}$ expression in A549 cells, with $\beta 6$ subunit reached a peak level at $6 \mathrm{~h}$ (3.8 folds increase) which decreased thereafter. However, $\alpha \mathrm{v}$ integrin subunit mRNA expression persisted for $12 \mathrm{~h}$. The protein expression levels of $\alpha \mathrm{v}$ and $\beta 6$ subunits showed a gradual increase in response to IL- $1 \beta$ stimulation. Quiescent A549 cells showed constitutive $\alpha \mathrm{v}$ and $\beta 6$ subunit integrin mRNA and protein expression. The increase in $\alpha \mathrm{v}$ and $\beta 6$ expression levels at mRNA and protein levels signifify the role played by the integrin receptors in mediating the pulmonary edema.

\subsubsection{Cell Internalization of MPS-L ${ }^{\text {cRGD }}$ : CLSM Studies}

To evaluate the cell internalization of MPS-L ${ }^{\text {cRGD }}$, A549 cells were analyzed by CLSM studies. Either quiescent or activated A549 cells were incubated with MPS-L ${ }^{\text {cRGD }}$ or MPS-L ( $1 \mathrm{mM}$ lipid $/ 10^{6}$ cells $/ \mathrm{mL}$ ) for $1 \mathrm{~h}$ at $37^{\circ} \mathrm{C}$. As represented in Figure.6.2, activated A549 cells incubated with MPS-L ${ }^{\text {cRGD }}$ resulted in NBD fluorescence which is about 3-4 times higher than with MPS-L. In contrast, quiescent A549 cells incubated with MPS-L ${ }^{\text {cRGD }}$ showed a minimal level of NBD fluorescence over activated A549 cells. The fluorescence intensity of MPS-L emitted from either quiescent or IL-1 $\beta$ activated A549 cells was comparable, but insignificant over the background. The results indicate the higher affinity of MPS-L ${ }^{\text {cRGD }}$ towards activated A549 cells. cRGD peptide is known to bind to many heterodimer integrins, such as $\alpha v \beta 3, \alpha v \beta 5$ and $\alpha 5 \beta 1$ while showing minimal effects on $\alpha v \beta 6$ integrin receptors. Therefore, A549 cells were treated with integrin antibodies other than anti- $\alpha \mathrm{v} \beta 6$ integrin antibody prior to incubation with MPS$\mathrm{L}^{\mathrm{cRGD}}$

\subsubsection{Effect of MPS-L ${ }^{\text {cRGD }}$ on MCP-1 Expression in A549 Cells}

MCP-1 expression in alveolar epithelial cells plays a critical role in monocyte recruitment from blood circulation into alveolar spaces. Earlier studies have shown that IL-1 $\beta$ is a potent stimulus for MCP-1 expression in both primary and transformed epithelial cells. To examine the MCP-1 expression in IL-1 $\beta$ activated A549 cells, ELISA was performed. As demonstrated in Figure 6.3, MCP-1 protein was constitutively expressed in quiescent cells. In IL-1 $\beta$ activated cells, a steady and significant $(\mathrm{p}<0.001)$ increase in MCP-1 protein expression was observed till $6 \mathrm{~h}$, and persisted thereafter (data not shown). To investigate whether the effect of IL-1 $\beta$ stimulation on MCP-1 expression would be inhibited by MPS, A549 cells were pretreated with different control and MPS encapsulated formulations containing an MPS equivalent to $30 \mu \mathrm{M}$, before stimulation with IL-1 $\beta(10 \mathrm{ng} / \mathrm{mL})$ for $12 \mathrm{~h}$. 


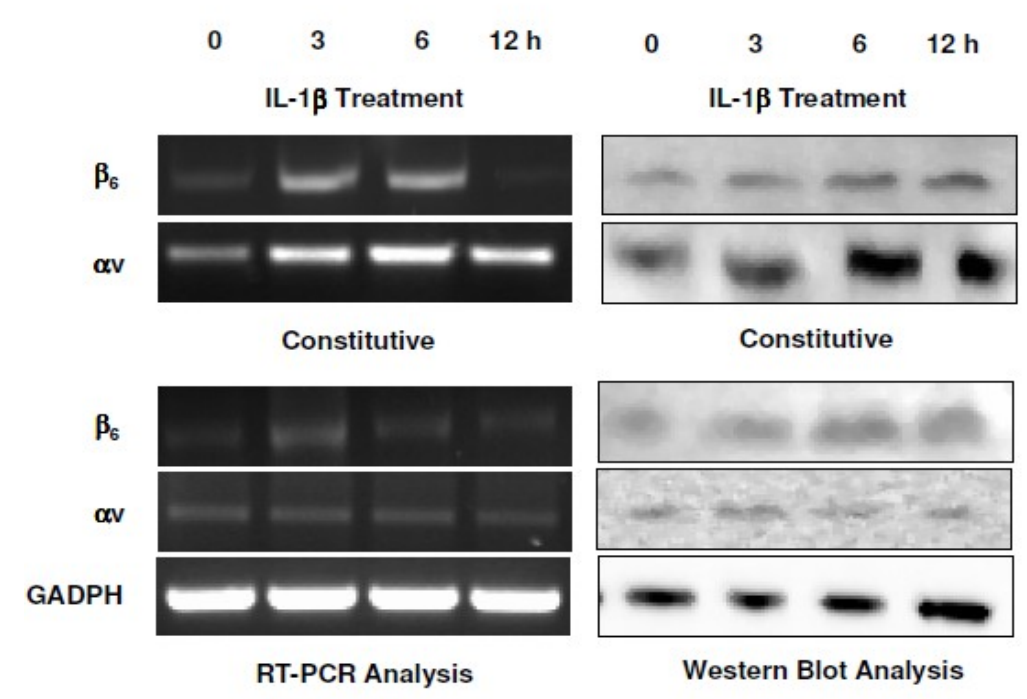

Figure 6.1 RT-PCR and Western Blot analyses of concentration dependent inhibition of $\alpha v \beta 6$ integrin expression by MPS.

After the indicated treatments as described in section, 6.2.2.3, cellular lysates from the experiments were subjected to Western Blot and RT-PCR analyses of integrin subunits and GADPH. For each set of conditions, three blots were conducted and representatives were shown. All the blots are a representative of $n=3$ samples. 


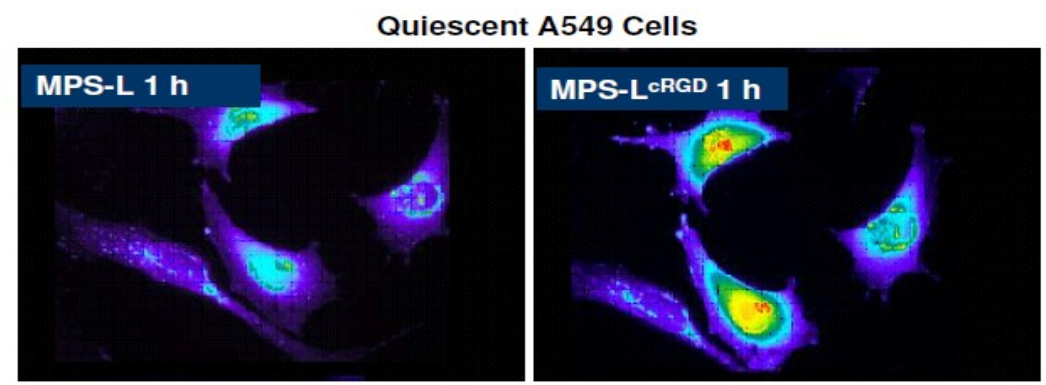

IL-1ß activated A549 Cells

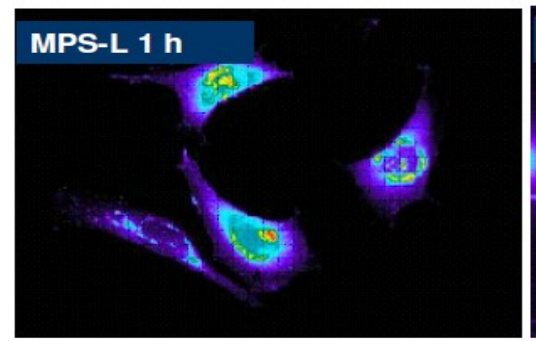

MPS-LCRGD 1 h

Figure 6.2 Confocal laser fluorescence microscopy of cell internalization of MPS-L and MPS-L ${ }^{\text {cRGD }}$ in quiescent and IL-1 $\beta$ activated A549 cells.

A549 cells were treated with IL-1 $\beta(10 \mathrm{ng} / \mathrm{mL})$ prior to treatments with formulations. After the treatments, unbound liposomes were washed with citrate buffer and cells were observed under 100,000x magnification. All the liposome treatments were performed at $37^{\circ} \mathrm{C}$ for $1 \mathrm{~h}$. Top panel shows the fluorescence emitted from quiescent cells and lower panel shows that from IL-1 $\beta$ treated cells. 

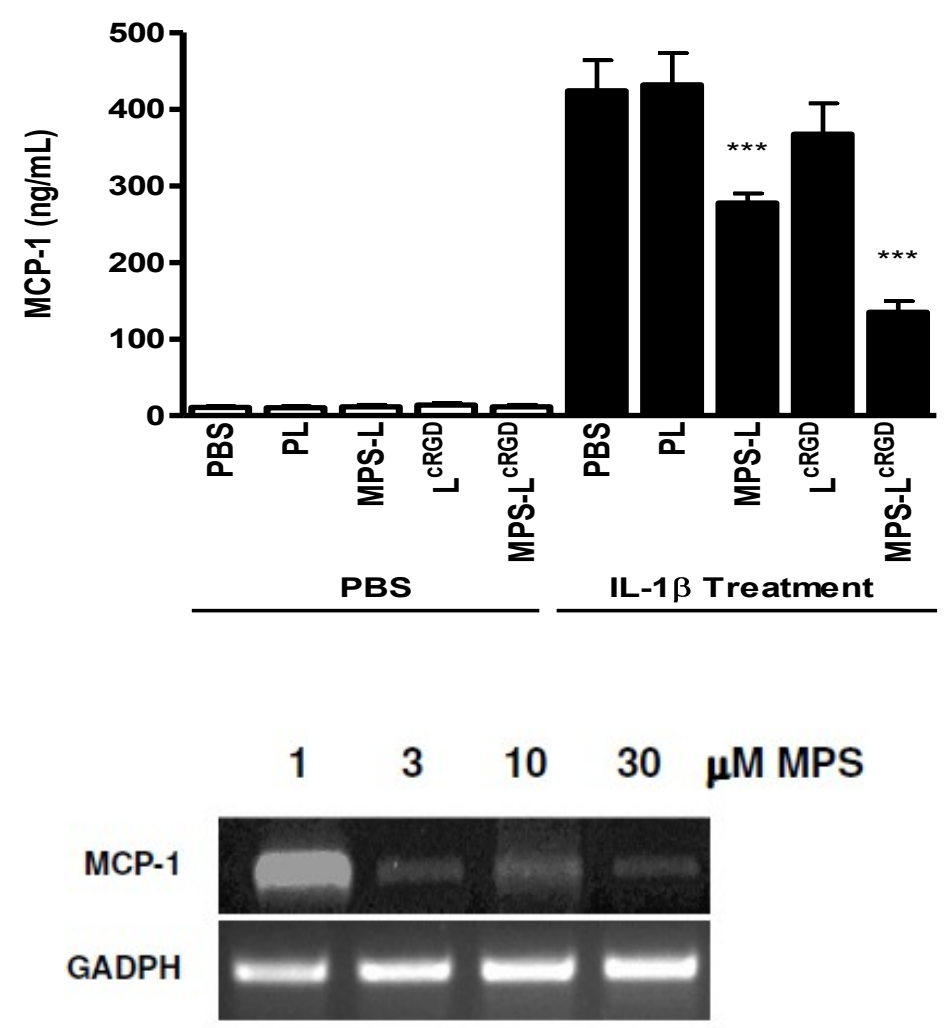

RT-PCR analysis

Figure 6.3 ELISA and RT-PCR analyses of MCP-1 expression in A549 cells as a function of MPS encapsulated formulations.

A549 cells were pre-incubated with MPS-L and MPS-L ${ }^{\text {cRGD }}$ (30 $\mu$ M MPS equivalents). Followed by this, cells were treated with IL- $1 \beta$ at $37^{\circ} \mathrm{C}$ for $1 \mathrm{~h}$. Supernatants were collected for chemokines analysis and cells were harvested for RT-PCR analysis. Data are plotted in nanograms per milliliter as mean \pm SEM. $* * *$ indicates significant difference of MP-L ${ }^{\text {cRGD }}$ treatment effect at $\mathrm{P}<0.001$ from the IL- $1 \beta$ stimulation alone. The top panel shows the ELISA results of MCP-1 expression, while the lower panel represents the RTPCR analysis of the data. 
MPS-L ${ }^{\text {cRGD }}$ inhibited MCP-1 protein expression to $26 \%$ of the IL- $1 \beta$ stimulation alone. Similarly, MPS-L attenuated MCP-1 mRNA to $60 \%$ of IL- $1 \beta$ stimulated levels. The results suggest that MPS released from MPS-L ${ }^{\mathrm{cRGD}}$ attenuates IL-1 $\beta$ activated MCP-1 expression upon cell internalization. MPS-L ${ }^{\mathrm{cRGD}}$ treatments showed no effect on cell viability in 0.3-30 $\mu \mathrm{M}$ range (data not shown). The results from NF- $\kappa \beta$ EMSA and Western Blot analysis also corroborate the evidence that MCP-1 gene, controlled by NF$\kappa \beta$ was modulated using MPS formulation treatments, particularly, MPS- $\mathrm{L}^{\mathrm{cRGD}}$.

In summary, A549 cells instilled with IL-1 $\beta$ showed elevated mRNA and protein levels of MCP-1. Compared with the control, MPS containing formulations treatment reduced mRNA and protein levels of MCP-1 after IL-1 $\beta$ administration.

\subsubsection{Effect of MPS-L ${ }^{\text {cRGD }}$ on Monocytes Adhesion to IL-1 $\beta$ Stimulated A549 Monolayers}

An IL-1 $\beta$ concentration dependent monocytes adhesion to A549 cells was observed. Monocytes adhesion to activated A549 cells increase by $280 \%$ corresponding to 10 $\mathrm{ng} / \mathrm{mL}$ of IL-1 $\beta$ dose, respectively. To examine the effect of MPS on monocyte adhesion, A549 cells were pretreated with MPS- $\mathrm{L}^{\mathrm{cRGD}}$ (equivalent to $30 \mu \mathrm{M}$ MPS) for $12 \mathrm{~h}$ before IL-1 $\beta(10 \mathrm{ng} / \mathrm{mL})$ stimulation for $6 \mathrm{~h}$. MPS-L ${ }^{\text {cRGD }}$ treatment showed a significant decrease in monocyte adhesion to activated A549 cells. As shown in Figure 6.4, at concentration equivalent to $30 \mu \mathrm{M}$ MPS, monocyte adhesion to activated A549 cells was reduced to near basal levels. To examine the effect of $\beta 1$ or $\beta 6$ antibodies, anti- $\beta 1$ or anti$\beta 6$ were used, it is evident anti- $\beta 1$ modulates monocytes adhesion to IL- $1 \beta$ in a mildly.

\subsubsection{Effect of MPS-L ${ }^{\text {cRGD }}$ on ICAM-1 Expression in A549 Cells}

Cell adhesion mediated by ICAM-1 is critical for the transendothelial migration of neutrophils from blood circulation into alveolar spaces. In ALI, interaction between pathogens and macrophages or leukocytes results in release of cytokines, causing lung damage. In vivo, enhanced expression of lung ICAM-1 is presumed to be the result of direct inflammatory stimuli in concert with secondary effects (i.e., cytokines and chemokines) mediated by inflammatory cells. In this study, the direct effects of IL-1 $\beta$ on ICAM-1 expression in A549 epithelial cell line are evaluated. Alveolar epithelium shows constitutive ICAM-1 expression, which is upregulated in response to pro-inflammatory mediators, such as IL-1 $\beta$ and TNF- $\alpha$. To examine the ICAM-1 expression, A549 cells were treated with IL-1 $\beta(10 \mathrm{ng} / \mathrm{mL})$ for $0,3,6$ and $12 \mathrm{~h}$. IL-1 $\beta$ induced a gradual and significant $(\mathrm{P}<0.001)$ increase in $\mathrm{mRNA}$ and protein expression compared to the constitutive expression of quiescent cells (data not shown). Activated A549 cells showed a maximal, $243 \%$ increase in mRNA expression at $6 \mathrm{~h}$ followed by a slight decrease thereafter. 


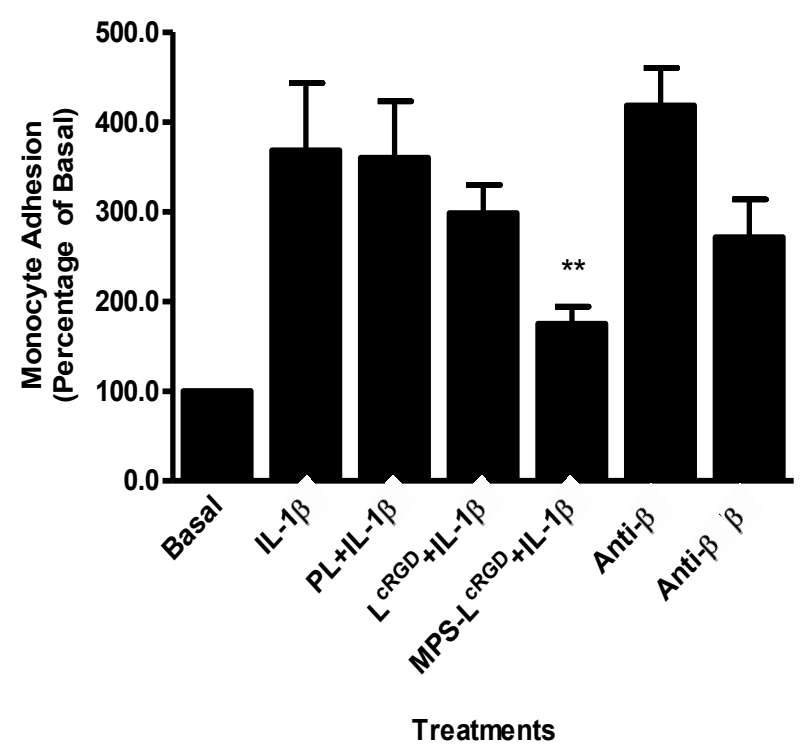

Figure 6.4 Histogram of the monocyte adhesion as a function of MPS encapsulated formulation treatments in IL-1 $\beta$ activated A549 cells.

Monocyte adhesion is expressed as percentage of monocytes adhered to A549 cells in basal and stimulated conditions (IL-1 $\beta$ ). Data are plotted mean \pm SEM, $n=3$ samples. ** indicates significant $(\mathrm{P}<0.01)$ inhibition of monocyte adhesion with MPS-L ${ }^{\text {CRGD }}$ in comparision with IL-1 $\beta$ stimulation alone. 
To investigate the inhibitory potential of MPS on IL-1 $\beta$ induced ICAM-1 up-regulation, A549 cells were pre-incubated with MPS- ${ }^{\text {cRGD }}$ (equivalent to $0.03-30 \mu \mathrm{M}$ of MPS) for $12 \mathrm{~h}$ before stimulation with IL-1 $\beta(10 \mathrm{ng} / \mathrm{mL})$ for $6 \mathrm{~h}$. Figure 6.5 shows that MPS inhibited the up-regulation of ICAM-1 protein expression in a concentration-dependent manner. At $30 \mu \mathrm{M}$ concentration, MPS attenuated ICAM-1 production to $18 \%$ of IL- $1 \beta$ stimulation alone. To further confirm the inhibitory effect of MPS-L ${ }^{\text {CRGD }}$ on IL-1 $\beta$ induced ICAM-1 gene expression, the mRNA levels were analyzed by RT-PCR. A549 cells were subjected to the similar treatment as described above. MPS released from MPS-L ${ }^{\text {cRGD }}$ abrogated IL-1 $\beta$ induced ICAM- 1 mRNA expression in a dose dependent manner (data not shown). It was observed that at $30 \mu \mathrm{M}$ concentration, MPS attenuated $82 \%$ of IL- $1 \beta$ activated mRNA up-regulation. From the results, it was found that MPS released from MPS-L ${ }^{\mathrm{CRGD}}$ significantly attenuated IL-1 $\beta$ induced ICAM-1 production in A549 cells, both at the protein and mRNA levels.

\subsubsection{Effect of MPS- $\mathrm{L}^{\mathrm{cRGD}}$ on Neutrophil Adhesion to A549 Monolayers}

IL-1 $\beta$ stimulation mediates the adherence of neutrophils to epithelial cells through upregulation of ICAM-1 receptors. A549 cells were exposed to IL-1 $\beta$ for $6 \mathrm{~h}$. After the monolayers were washed, neutrophils were added and incubated for $20 \mathrm{~min}$. Nonadherent neutrophils were washed away. Neutrophils adhesion increase by $48 \%, 210 \%$, and $385 \%$ on exposure of A549 cells to $0.1,1$ and $10 \mathrm{ng} / \mathrm{mL}$ of IL- $1 \beta$, respectively (data not shown). To investigate the effect of MPS on neutrophils adhesion, A549 cells were pretreated with MPS- ${ }^{\mathrm{CRGD}}$ (equivalent to $30 \mu \mathrm{M}$ of MPS) containing liposome formulations for $12 \mathrm{~h}$ before IL-1 $\beta$ treatment for $6 \mathrm{~h}$. As shown in Figure 6.6, neutrophil adhesion was reduced to $33 \%$ of IL- $1 \beta$ stimulation alone. A combination of antibodies to $\beta 1$ and $\beta 6$ significantly reduced the neutrophil adhesion to A549 cells. Anti- $\beta 1 / \beta 6$ antibodies combination contributed a synergistic effect on inhibition of neutrophil adhesion. This study reveals the involvement of both $\beta 1$ and $\beta 6$ integrins in mediating the neutrophil migration into alveoli.

\subsubsection{Effect of MPS-L ${ }^{\text {cRGD }}$ on Epithelial Permeability in IL-1 $\beta$ Induced A549 Cells}

IL-1 $\beta$ have been reported to mediate epithelial barrier dysfunction via $\alpha v \beta 6$ integrins, we therefore analyzed the potential of MPS- $\mathrm{L}^{\mathrm{CRGD}}$ on the modulation of epithelial cell permeability. Confluent A549 cells on permeable supports were incubated with IL-1 $\beta$ and transepithelial permeability was determined by measurement of ${ }^{14} \mathrm{C}$-albumin flux. IL-1 $\beta$ increased permeability in A549 cells compared to control. The transepithelial albumin flux increased $220 \%$ following IL- $1 \beta$ treatment. However, MPS-L ${ }^{\text {CRGD }}$ (equivalent to 30 $\mu \mathrm{M}$ MPS) pretreatment reduced the transepithelial permeability of activated A549 cells to basal levels $(\mathrm{P}<0.001)$. To delineate the effect of $\mathrm{L}^{\mathrm{CRGD}}$ from MPS on transepithelial permeability, A549 cells were pretreated with $\mathrm{L}^{\text {cRGD }}$ (equivalent to $33.4 \mu \mathrm{g} / \mathrm{mL}$ of cRGDpeptide), which significantly $(\mathrm{P}<0.05)$ reduced the ${ }^{14} \mathrm{C}$-albumin flux to near basal levels. It has been shown that IL-1 $\beta$ increases alveolar epithelial permeability by activating 

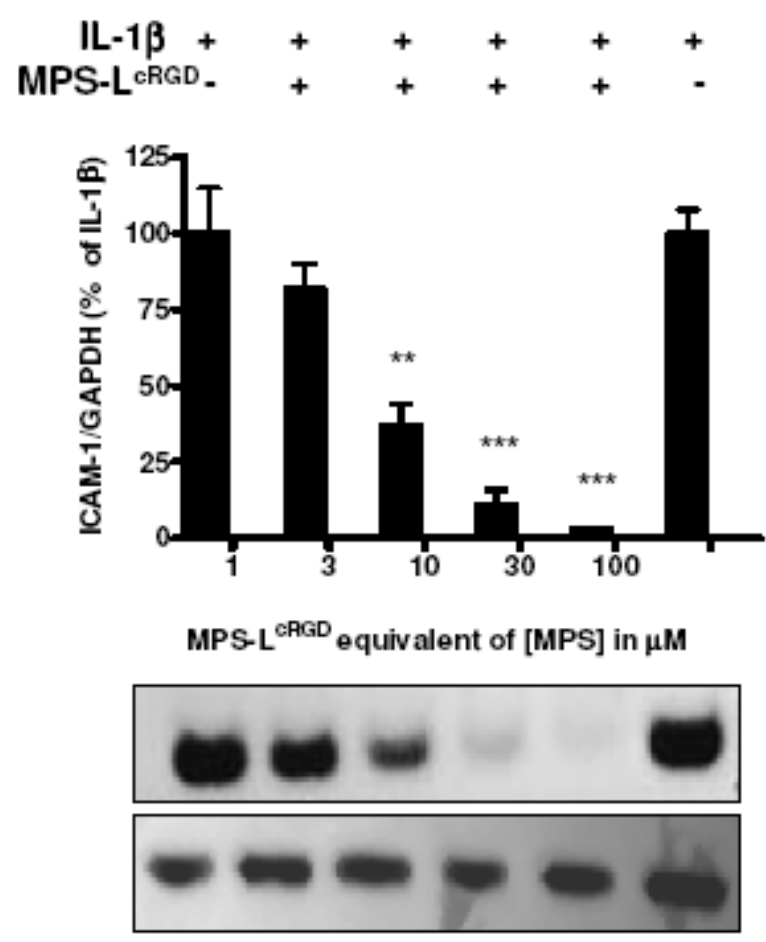

Figure 6.5 Densitometric RT-PCR and Western Blot analyses of ICAM-1 expression in A549 cells, treated with MPS-L ${ }^{\text {RGD }}$.

MPS inhibits the expression of ICAM-1 induced by IL-1 $\beta$. A549 cells were preincubated with MPS-L ${ }^{\text {cRGD }}$ (1-100 $\mu$ M MPS equivalents). After $12 \mathrm{~h}$, supernatants were collected for ICAM-1 analysis and cells were harvested for RT-PCR analysis. Lower panel represents the Western Blot analysis and top panel represents the densitometric RTPCR analysis of ICAM-1 expression. ${ }^{* *}$ and $* * *$ indicate significant levels at $\mathrm{P}<0.01$ and 0.001 , respectively from the IL- $1 \beta$ stimulated levels alone. 


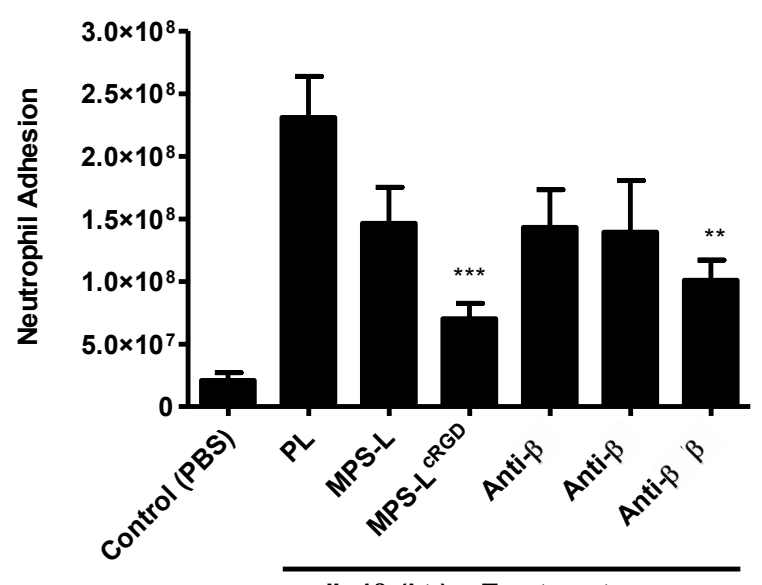

IL-1 $\beta$ (i.t.) + Treatment

Figure 6.6 Histogram of neutrophil adhesion as a function of MPS encapsulated formulation treatments in IL-1 $\beta$ activated A549 cells.

Freshly isolated neutrophils were isolated and incubated with quiescent and stimulated A549 cells. A549 cell monolayers were pretreated with MPS-L ${ }^{\text {cRGD }}$ (MPS equivalent to $30 \mu \mathrm{M})$ and antibody $(10 \mu \mathrm{g} / \mathrm{mL})$. Data are shown as mean \pm SEM. $* *$ and $* * *$ indicate $\mathrm{P}<0.01$ and $\mathrm{P}<0.001$, respectively from IL- $1 \beta$ stimulated levels alone. 
$\alpha v \beta 6$ integrin receptors in alveolar type II cells. Therefore, we examined their role using anti- $\alpha \mathrm{v} \beta 6$ antibodies. A549 cells were pretreated with anti- $\alpha \mathrm{v} \beta 6(20 \mu \mathrm{g} / \mathrm{mL})$ antibodies before IL- $1 \beta$ treatment. ${ }^{14} \mathrm{C}$-albumin flux was reduced to basal levels across the A549 cells (Figure 6.7). The data indicates the role of $\alpha v \beta 6$ integrin receptors in mediating the epithelial cell permeability increase in IL-1 $\beta$ stimulated A549 cells. It is also evident that $\mathrm{L}^{\mathrm{cRGD}}$ reduce the transepithelial albumin flux by blocking the $\alpha \mathrm{v} \beta 6$ integrin receptors. From the results, it is evident that the targeting ligand blocks the integrin receptors and seems to prevent the interaction with LAP doman of the TGF- $\beta$-LAP complex. In both $\mathrm{L}^{\mathrm{cRGD}}$ and MPS- $\mathrm{L}^{\mathrm{cRGD}}$ formulations, a significant reduction was observed. However, a mild reduction in epithelial permeability was observed with MPS-L. The data signifies the importance of integrin receptors in mediating the IL-1 $\beta$ stimulated epithelial permeability.

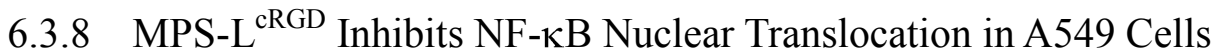

NF- $\kappa \mathrm{B}$ is a major transcription factor mediating IL-1 $\beta$ induced MCP-1 and ICAM-1 expression through the NF- $\kappa \mathrm{B}$ binding sites located in the enhancer region of the MCP-1 and ICAM-1 genes, respectively. To explore the inhibition potential of MPS-L ${ }^{\mathrm{cRGD}}$, we used EMSA to determine whether MPS released from targeted liposomes could affect $\mathrm{NF}-\kappa \mathrm{B}$ activation in A549 cells. Figure 6.8 demonstrates $\mathrm{NF}-\kappa \mathrm{B}$ activity in response to IL-1 $\beta$ exposure.

Since activation of NF- $\kappa \mathrm{B}$ in A549 cells is dependent on phosphorylation and subsequent degradation of I $\mathrm{I} B \alpha$, cytoplasmic extracts from IL-1 $\beta$ activated A549 cells in the presence or absence of MPS- $\mathrm{L}^{\mathrm{cRGD}}$ was examined by Western blot analysis. In quiescent cells, low levels of phosphorylated $I_{\kappa} B \alpha\left(p-I_{\kappa} B \alpha\right)$ were detected. However, $p-I_{\kappa} B \alpha$ was readily detectable following IL- $1 \beta$ treatment. Prior incubation with MPS-L ${ }^{\text {cRGD }}$ led to a concentration dependent inhibition of $\mathrm{p}-\mathrm{I} \kappa \mathrm{B} \alpha$. A549 cells pretreatment with MPS-L ${ }^{\text {cRGD }}$ for $12 \mathrm{~h}$ caused a substantial accumulation of $\mathrm{I} \kappa \mathrm{B} \alpha$ (data not shown) and reduced $\mathrm{p}-\mathrm{I} \kappa \mathrm{B} \alpha$ levels compared with IL-1 $\beta$ alone. However, short interval pretreatment with MPS-L ${ }^{\text {cRGD }}$ did not alter $I_{\kappa} B \alpha$ (data not shown) and $p-I_{\kappa} B \alpha$ levels. The data indicates that MPS released from MPS-L ${ }^{\mathrm{cRGD}}$ inhibits NF- $\kappa \mathrm{B}$ transcriptional activity, at least in part, by increasing I $\mathrm{B} \mathrm{B} \alpha$ due to reduced I $\kappa \mathrm{B} \alpha$ phosphorylation and degradation.

The data indicates that the targeting of NF- $\kappa$ B dependent transcription by MPS represents a major mechanism of anti-inflammatory action. In support of this statement, MPS treatment modulated activated NF- $\kappa$ B in A549 epithelial cells. Furthermore, we now report that the induction of $\mathrm{NF}-\kappa \mathrm{B}$ dependent transcription A549 epithelial cells was affected by MPS in a concentration dependent manner. Therefore, higher concentrations of MPS or longer incubation times are necessary to exert the inhibitory effects on A549 cells. Consistent with this, there was noticeable effect of MPS on the expression of MCP1 and ICAM-1, where inhibition of $\mathrm{p}-\mathrm{I} \kappa \mathrm{B} \alpha$ resulted in a profound repression of MCP-1 and ICAM-1 expression. Thus, NF- $\mathrm{\kappa B}$ inhibitors seem to target processes related to 


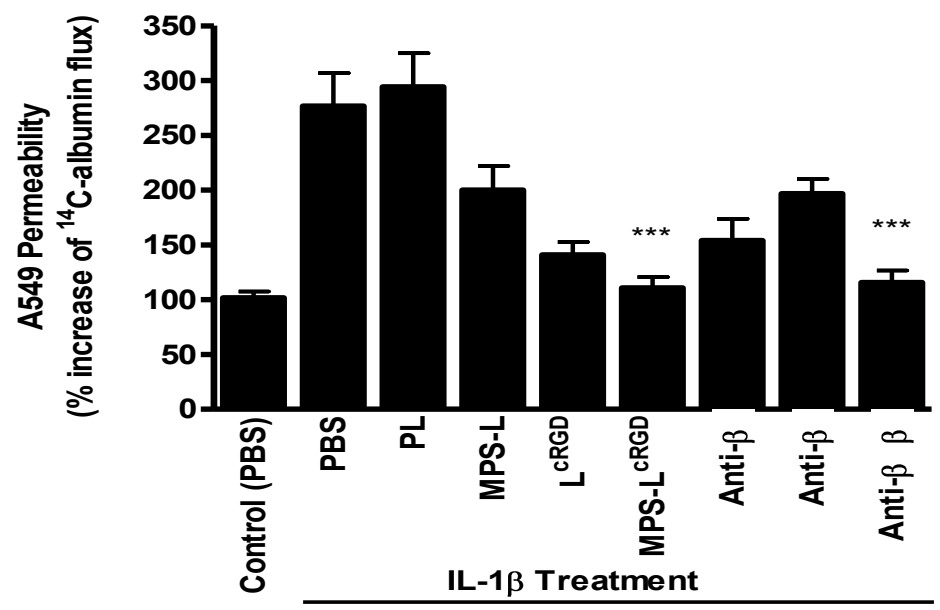

Figure 6.7 Histogram of ${ }^{14} \mathrm{C}$-albumin permeability across $\mathrm{A} 549$ cells, pretreated with MPS encapsulated formulations and antibodies.

Data are shown as mean \pm SEM. $* * *$ indicates $\mathrm{P}<0.001$ from IL-1 $\beta$ stimulated levels alone. 


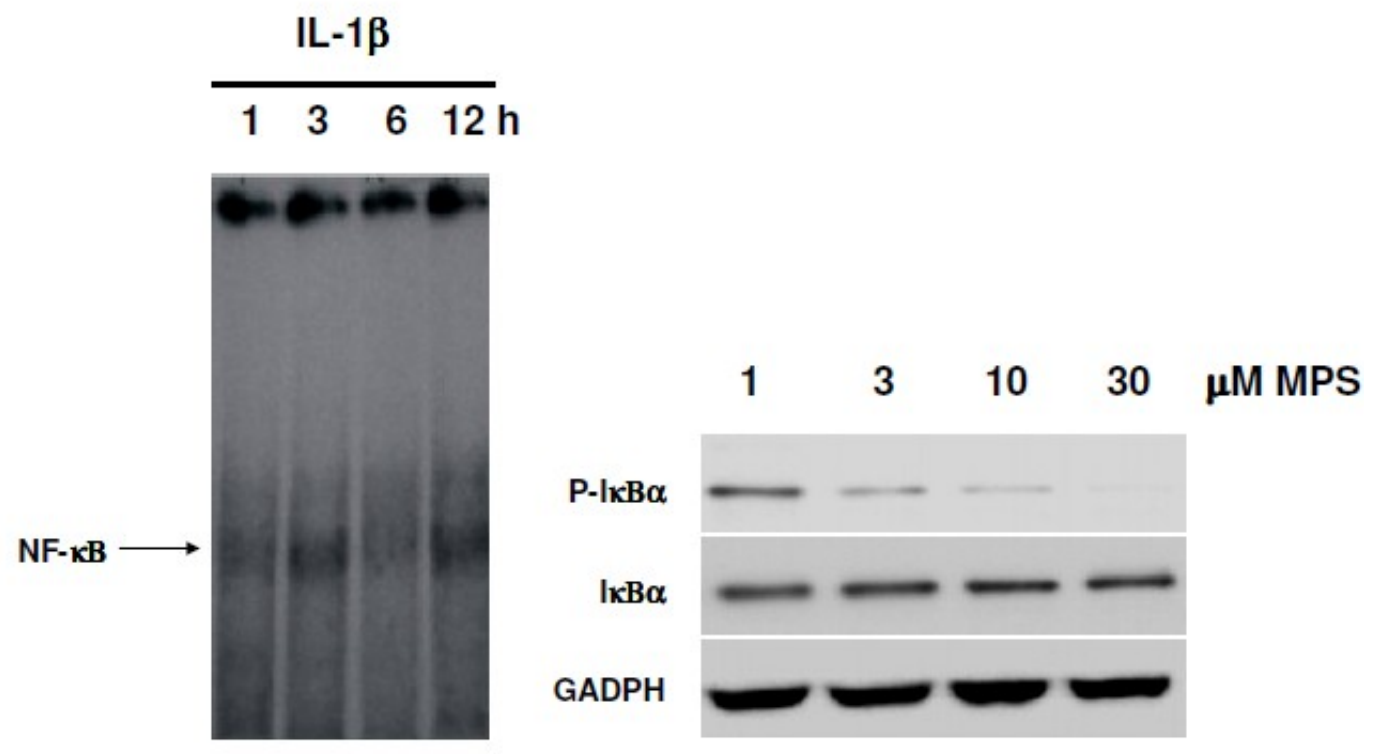

Figure 6.8 EMSA (left panel) of NF- $\kappa$ B elicitation and Western Bot analysis of I $\kappa \mathrm{B} \alpha$ and $\mathrm{p}-\mathrm{I} \kappa \mathrm{B} \alpha$ expression pattern in A549 cells, pretreated with MPS-L ${ }^{\mathrm{cRGD}}$ formulation.

MPS attenuated stimulus loss of I $\mathrm{B} \alpha$. A549 cells were pretreated for $12 \mathrm{~h}$ with the MPS-L ${ }^{\text {cRGD }}$. Cells stimulated with IL- $1 \beta$ were harvested. NF- $\kappa B$ activity was analyzed by EMSA (left panel) on nuclear extracts. A Western Blot analysis (right panel) of total $\mathrm{I}_{\kappa} \mathrm{B} \alpha$ and $\mathrm{p}-\mathrm{I} \kappa \mathrm{B} \alpha$ was performed on cytoplasmic extracts. All blots are representative of three experiments. 
corticosteroids in the repression of inflammatory gene expression. This finding may have a considerable impact in the context of steroid insensitive-ALI.

In summary, we demonstrate that targeting NF- $\kappa \mathrm{B}$ inhibitors with small molecule inhibitors is effective at preventing the activation of NF- $\kappa \mathrm{B}$ in pulmonary epithelial cells. Furthermore, this manipulation results in the reduced expression of proinflammatory genes in both A549 cells. Based on the inflammatory roles of genes, such as ICAM-1, IL6 and MCP-1, and the key role of the alveolar epithelium in the pathogenesis of $\mathrm{ALI} / \mathrm{ARDS}$, we predict that inhibitors of NF- $\kappa \mathrm{B}$ may represent valuable antiinflammatory agents in management of ALI.

\subsubsection{Effect of MPS-L ${ }^{\text {cRGD }}$ on BALF Protein Concentration in IL-1 $\beta$ Induced Rats}

Quantification of BALF protein concentration is considered as an indicator of increase in lung vascular and epithelial permeability resulting from inflammatory injury. In the present study, BALF protein concentration was quantified in control and treated groups. As represented in Figure 6.9, protein concentration in the BALF of IL-1 $\beta$ (i.t.) instilled group was nearly 3.6 folds that of rats receiving saline (i.t.) (IL-1 $\beta$ group, $0.43 \pm 0.07$ $\mathrm{mg} / \mathrm{mL}$; saline group, $0.12 \pm 0.02 \mathrm{mg} / \mathrm{mL} ; \mathrm{P}<0.001)$. A significant inhibition of BALF protein concentration was observed in rats pretreated with MPS- $\mathrm{L}^{\mathrm{cRGD}}(0.18 \pm 0.03$ $\mathrm{mg} / \mathrm{mL} ; \mathrm{P}<0.001$ ) compared to IL-1 $\beta$ (i.t.) instilled group. To delineate the effects of MPS and cRGD-peptide conjugated liposomes on the inhibition of BALF protein, rats were pretreated with MPS-L or $\mathrm{L}^{\mathrm{cRGD}}$, respectively. Reduction of BALF protein was observed in rats pretreated with either MPS-L $(0.28 \pm 0.06 \mathrm{mg} / \mathrm{mL} ; \mathrm{P}<0.05)$ or $\mathrm{L}^{\mathrm{cRGD}}$ $(0.21 \pm 0.02 \mathrm{mg} / \mathrm{mL} ; \mathrm{P}<0.01)$ formulation, but not as significant as MPS-L ${ }^{\mathrm{cRGD}}$. The data suggests the additive effect of MPS-L ${ }^{\mathrm{cRGD}}$ in reducing the BALF protein content.

\subsubsection{Effect of MPS-L ${ }^{\text {CRGD }}$ on MCP-1 Production in BALF of IL-1 $\beta$ Induced Rats}

Since IL-1 $\beta$ induced macrophage and neutrophil migration is largely determined by MCP-1 expression, we measured the BALF levels at $24 \mathrm{~h}$ following IL-1 $\beta$ (i.t.) instillation in pretreated (MPS- $\mathrm{L}^{\mathrm{cRGD}}$, MPS-L or $\mathrm{L}^{\mathrm{cRGD}}$ ) rat groups. Saline and IL-1 $\beta$ alone instilled groups were treated as negative and positive control groups. MPS-L ${ }^{\text {cRGD }}$ treatment resulted in decrease of MCP-1 $(1537.4 \pm 65.4 \mathrm{pg} / \mathrm{mL}$ in IL-1 $\beta$ alone instilled rats vs. $523.2 \pm 92.1 \mathrm{pg} / \mathrm{mL}$ in MPS-L ${ }^{\text {cRGD }}$ treated rats, $\left.\mathrm{P}<0.001\right)$ and the levels were comparable to basal levels as that of saline instilled negative control group (Figure 6.10). MPS-L pretreated group also showed a significant decrease in MCP-1 (987.4 \pm 74.1 $\mathrm{pg} / \mathrm{mL}$ ) in IL- $1 \beta$ instilled rats. While $\mathrm{L}^{\mathrm{cRGD}}$ has no influence on MCP-1 production, whose levels were similar to that of IL-1 $\beta$ instilled positive control group. 


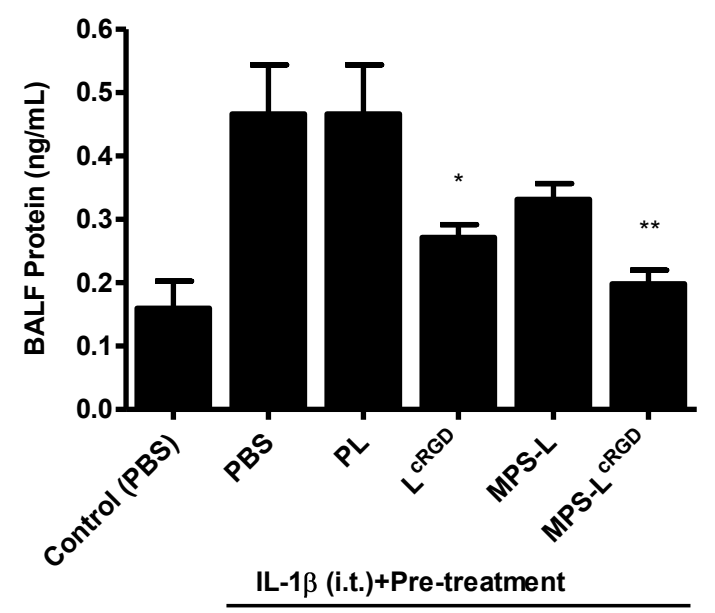

Figure 6.9 Histogram depicting the inhibition of BALF protein after nebulization of MPS-L ${ }^{\text {cRGD }}$ in male Sprague-Dawley rats.

BALF protein levels were measured by Bio-Rad micro-protein assay. Results are expressed as mean \pm SEM of four experiments. * and ** indicate statistically signigicant levels at $\mathrm{P}<0.05$ and 0.01 levels, respectively from IL-1 $\beta$ stimulated levels alone.

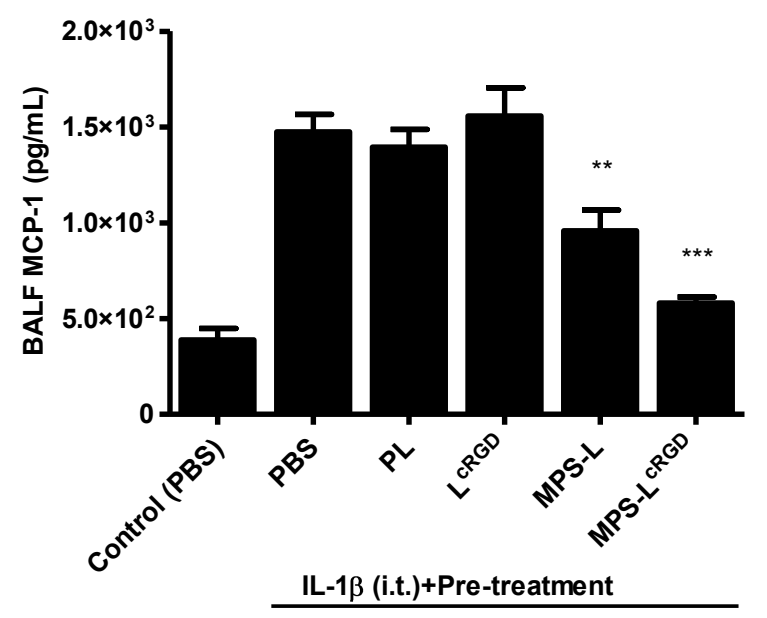

Figure 6.10 Histogram of the inhibition of BALF MCP-1 levels after nebulization of MPS-L ${ }^{\text {cRGD }}$ in male Sprague-Dawley rats.

MCP-1 levels were measured by ELISA. Results are expressed as a mean \pm SEM of four experiments. $* *$ and $* * *$ indicate statistically signigicant levels at $\mathrm{P}<0.01$ and $\mathrm{P}<0.001$ levels, respectively from IL-1 $\beta$ stimulated levels alone. 


\subsubsection{Effect of MPS-L ${ }^{\text {cRGD }}$ on TNF- $\alpha$ Production in BALF of IL- $1 \beta$ Induced Rats}

As compared with saline-treated controls, IL-1 $\beta$ (i.t.) instillation induced a significant increase in BALF levels of TNF- $\alpha$ (Figure 6.11). After MPS- $\mathrm{L}^{\mathrm{cRGD}}$ treatment, BALF levels of TNF- $\alpha$ were significantly $(\mathrm{P}<0.001)$ lower and comparable to saline treated rats. Animals pretreated with MPS solution had lower levels of TNF- $\alpha$ but not as significant $(\mathrm{P}<0.01)$ as animals pretreated with MPS- $\mathrm{L}^{\mathrm{CRGD}}$. In contrast, BALF levels of TNF- $\alpha$ in animals pretreated with $\mathrm{L}^{\mathrm{cRGD}}$ had no effect on TNF- $\alpha$ production and their levels were similar to IL-1 $\beta$ (i.t.) instilled positive control group.

\subsubsection{Effect of MPS-L ${ }^{\mathrm{CRGD}}$ on Neutrophil Migration of IL-1 $\beta$ Induced Rats}

A variety of stimuli induce neutrophil migration into the lung. Adhesion molecules and chemokines have been shown to be involved. Chemokines, such as MCP-1, promote neutrophil migration into the alveolar compartment. Also, ICAM-1 gradient across the alveolar barrier is thought to be required for neutrophil sequestration into the vascular compartment. For quantitative assessment of neutrophil migration into alveolar spaces, MPO assay of BALF was performed in pretreated rat groups (MPS-L ${ }^{\mathrm{cRGD}}$, MPS-L or $\mathrm{L}^{\mathrm{CRGD}}$ ) post IL-1 $\beta$ (i.t.) instillation.

As illustrated in Figure 6.12, pretreatment of rats with MPS-L inhibited the neutrophil migration into alveolar spaces of rats receiving IL-1 $\beta$ (i.t.) as compared with saline treated rats $\left(1.9 \pm 0.1 \times 10^{6} ; \mathrm{p}<0.05\right.$ in MPS-L treated group vs $2 \pm 0.2 \times 10^{5} ; \mathrm{P}<0.05$ in saline treated group).Inhibition of neutrophil migration was further enhanced $\left(1.1 \pm 0.05 \times 10^{6}\right.$; $\mathrm{P}<0.01)$ with MPS- $\mathrm{L}^{\mathrm{CRGD}}$ as compared to MPS-L solution or $\mathrm{L}^{\mathrm{CRGD}}$ pretreated groups. Further, inhibition of BALF neutrophils significantly increased in rats pretreated with anti- $\beta 1 / \beta 6\left(1.4 \pm 0.05 \times 10^{6} ; \mathrm{P}<0.01\right)$. As estimated by MPO assays, BALF neutrophil content was not significantly different from the neutrophil number in BALF determined by Histopaque ${ }^{\circledR}$ assay.

The reduction of lung neutrophil recruitment observed under conditions could in part be attributed to inhibition of ICAM-1 and MCP-1 levels, which possesses chemotactic activity for neutrophils. As well, reduced lung neutrophil accumulation mediated by ICAM-1 and MCP-1 may be a result of reduced intra-pulmonary production of TNF- $\alpha$. Instillation of IL-1 $\beta$ induced lung injury is the primary stimulus for the upregulation of the adhesion molecules on alveolar epithelium or endothelium. In this model, both ICAM-1 and MCP-1 are required for lung neutrophil recruitment. Thus, it is likely that reduced lung neutrophil recruitment is due to reduced pulmonary production of TNF- $\alpha$ and subsequent reduction in the up-regulation of adhesion molecules in the pulmonary vasculature. 


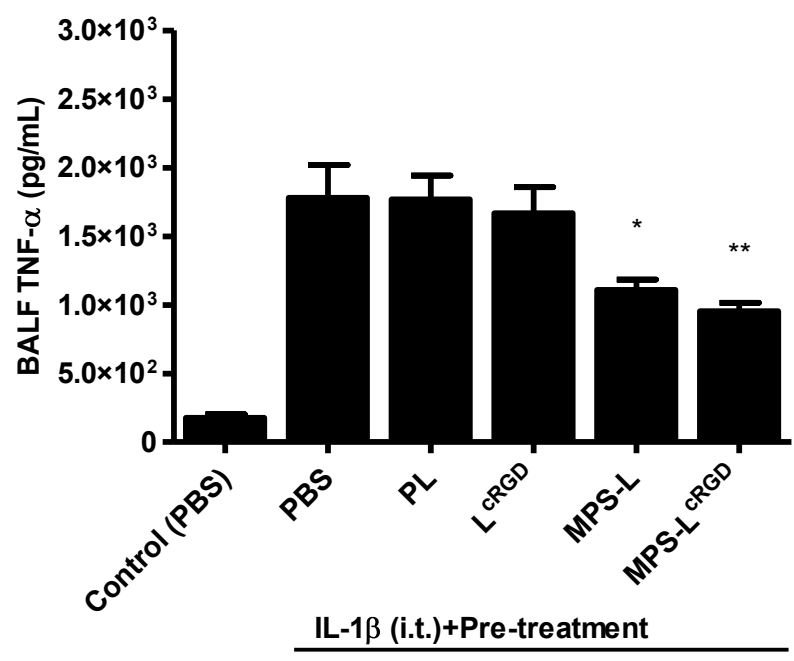

Figure 6.11 Histogram of inhibition of BALF TNF- $\alpha$ levels after nebulization of MPS$\mathrm{L}^{\mathrm{CRGD}}$.

TNF- $\alpha$ levels were measured by ELISA. Results are expressed a mean \pm SEM of four experiments. $*$ and $* *$ indicate significant difference at $\mathrm{P}<0.05$ and $\mathrm{P}<0.01$, respectively from IL-1 $\beta$ stimulated (PBS) alone.

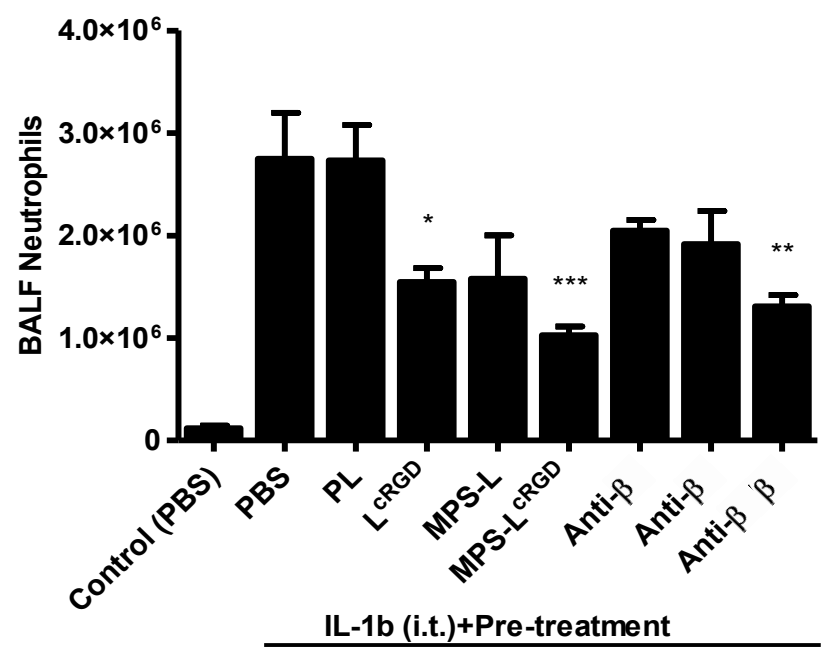

Figure 6.12 Histogram of inhibition of BALF levels of neutrophils after nebulization of MPS-L ${ }^{\text {CRGD }}$ in male Sprague-Dawley rats.

Neutrophils levels were analyzed by Histopaque and MPO assays. Results are expressed as a mean $\pm \mathrm{SEM}$ of four experiments. Statistically significant differences $(* \mathrm{P}<0.05, * *$ $\mathrm{P}<0.01$ and $* * * \mathrm{P}<0.001)$ compared with IL-1 $\beta$ treatment alone in each experiment. 


\subsubsection{Effect of MPS- $\mathrm{L}^{\text {cRGD }}$ on Neutrophil Accumulation in Lung Parenchyma}

Rats received IL-1 $\beta$ (i.t.) or saline as a control and were sacrificed $24 \mathrm{~h}$ later. As shown in Figure 6.13, rats receiving IL- $1 \beta$ showed a 6 fold increase $(\mathrm{P}<0.001)$ in lung parenchyma neutrophil content to $1.8 \pm 0.1 \times 10^{7}$ over saline control. Besides, BALF neutrophils in IL$1 \beta$ (i.t.) treated rats increased 14 folds $(\mathrm{P}<0.001)$ to $2.8 \pm 0.1 \times 10^{6}$ over control group. To investigate the effect of MPS- $\mathrm{L}^{\mathrm{cRGD}}$ on neutrophil migration into the lung during ALI, neutrophil accumulation which occurs prior to migration into alveoli was estimated by MPO assay of the lung tissue. Besides, haematoxylin staining techniques was adopted to visualize the neutrophil accumulation in paraffin embedded lung parenchyma sections. From the staining, it is evident that MPS- ${ }^{\mathrm{CRGD}}$ attenuated neutrophil accumulation in lungs compared to MPS-L or $\mathrm{L}^{\mathrm{CRGD}}$.

\subsection{Summary}

In summary, these studies demonstrate a critical role for the $\alpha v \beta 6$ integrins in mediating development of alveolar edema caused by IL-1 $\beta$, which is among the most biologically important inflammatory mediators in the airspace of patients with early ALI. We specifically used MPS encapsulated $\alpha v \beta 6$ specific integrin liposomes to deliver the drug to alveolar epithelial cells, the site of inflammation. In addition to inhibition of integrin

receptors, MPS- $\mathrm{L}^{\mathrm{CRGD}}$ inhibited inflammation and neutrophil migration in IL- $1 \beta$ instilled ALI model model. The inhibition of $\alpha \mathrm{v} \beta 6$ integrins blocked the IL- $1 \beta$ mediated protein permeability across the distal lung epithelium and prevented, in large part, the development of pulmonary edema. The findings reported here have potential clinical relevance as this mode of treatment may provide new causal therapies for pulmonary edema from ALI. 


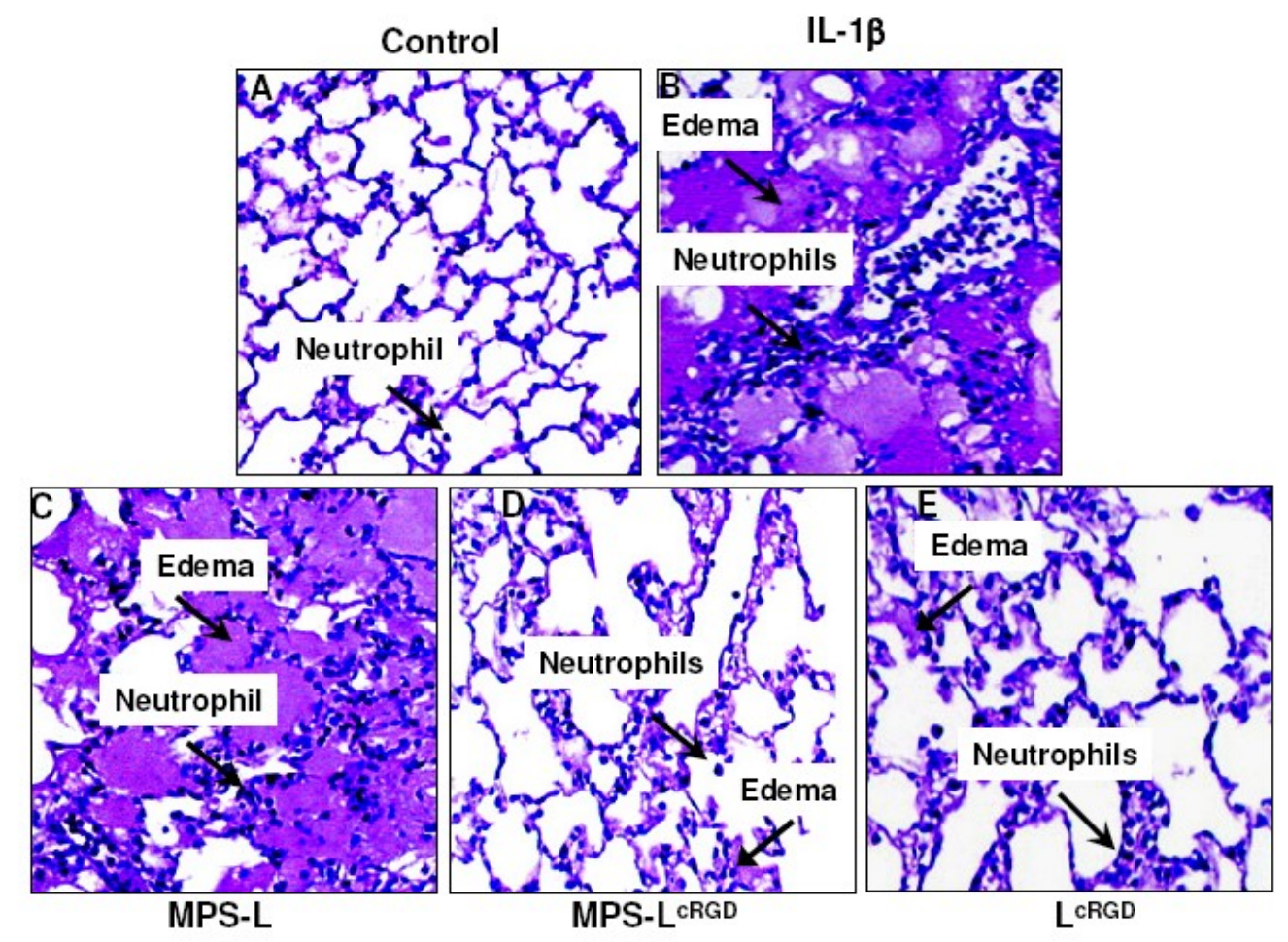

Figure 6.13 Histological sections of lung parenchyma after haematoxylin treatment to stain neutrophils.

Lung parenchyma sections represent the edema and neutrophil modulation pattern after MPS-L ${ }^{\text {cRGD }}$ pretreatment.Control (A) shows neat parenchyma cells without edema and a few neutrophils. IL-1 $\beta$ (B) instilled lung sections shows profuse edema and neutrophil migration. Non-specific liposomes $(C)$ exhibit profuse edema and a mild reduction in neutrophil infiltration. Among the lower panel, both MPS-L ${ }^{\text {cRGD }}$ (D) and $\mathrm{L}^{\mathrm{cRGD}}$ (E) showed reduction in neutrophil levels in alveoli. However, MPS-L ${ }^{\text {CRGD }}$ significantly reduced edema also. All the sections are a representative of $n=3$ samples. 


\section{CHAPTER 7. INHIBITION OF LUNG INFLAMMATION USING MPS-L ${ }^{\text {AB }}$}

\subsection{Introduction}

Acute lung injury and its more severe form, acute respiratory distress syndrome is characterized by inflammation at the alveolar-blood capillary interface, resulting in alveolar influx of protein rich edema fluid and consequently impairment in arterial oxygenation. ${ }^{4}$ ALI/ARDS is usually a major cause of acute respiratory failure with high morbidity and mortality in critically ill patients, affected by various predisposing insults. ${ }^{4,330}$ ALI usually arises within 4-72 $\mathrm{h}$ of the inciting insults and this period offers an opportunity for detection of inflammation and initiation of an effective treatment. ${ }^{331}$

The hallmark event in the pathophysiology of ALI/ARDS is the recruitment of a large number of neutrophils into alveoli with concomitant production of pro-inflammatory cytokines. ${ }^{16}$ Neutrophil recruitment from blood vasculature into alveolar spaces is orchestrated by sequential up-regulation of cell adhesion molecules (e.g. selectins, integrins and immunoglobulin supergene adhesion molecules) and their ligands, located on both vascular endothelial cells and neutrophils. ${ }^{332}$ The initial tethering and rolling of neutrophils over the endothelial cells is mediated by selectins and their ligands present on the apposing cells. During the rolling process, the integrin receptors on neutrophil surface undergo activation as a result of interaction with chemokines on the inflamed endothelium. The activated integrins further interact with immunoglobulin adhesion molecules (e.g. ICAM-1, VCAM-1), upregulated on the inflamed endothelium. These interactions result in firm adhesion of neutrophils to the endothelium. This is followed by the transendothelial migration of neutrophils from blood circulation into the alveolar sites of inflammation. ${ }^{333-335}$

In this multi-step process of neutrophil migration, firm adhesion is predominantly mediated by neutrophil $\beta 2$ integrins in conjunction with ICAM-1 of the endothelium. ${ }^{336}$ ICAM-1 is constitutively expressed on endothelial cells and its levels are upregulated in response to inflammatory stimuli, such as LPS, TNF- $\alpha$ and IL- $1 \beta .{ }^{337}$ The upregulation of ICAM-1 is usually long lasting with a maximum level at 4-6 $\mathrm{h}$ and remains elevated for $24 \mathrm{~h}^{338}$ This offers a potential opportunity for targeting to the endothelial ICAM-1 molecules using anti-ICAM-1 antibody as a specific targeting ligand. This strategy has been applied by conjugating anti-ICAM-1 monoclonal antibody onto the surface of liposomes.

IL-1 $\beta$ stimulates pro-inflammatory cytokine and chemokine release and upregulates CAMs in alveolar epithelial cells. ${ }^{320}$ Due to a high chemokine gradient across the alveolar barrier, leukocytes infiltrate from blood circulation into alveoli, and exacerbate edema and lung inflammation. ${ }^{321,322}$ Of the various transcriptional factors, $N F-\kappa B$ plays a central role in regulating the inflammatory genes encoding CAMs, chemokines and cytokines. ${ }^{75,323} \mathrm{NF}-\mathrm{\kappa B}$ is a heterodimeric protein present in the cytoplasm in an inactive form stabilized by binding to the inhibitory protein, $\mathrm{I} \kappa \mathrm{B} \alpha .{ }^{74}$ In response to IL-1 $\beta$ stimulation, IкB $\alpha$ undergoes phosphorylation and proteolytic degradation. As a result, p- 
$\mathrm{I} \kappa \mathrm{B} \alpha$ is ubiquitinated allowing nuclear translocation of $\mathrm{NF}-\kappa \mathrm{B}$, which binds to promoter regions of the target genes to initiate the transcription of CAMs, chemokines and proinflammatory cytokines. ${ }^{324,325}$ And inhibitors targeting the activated NF- $\mathrm{KB}$ would suppress CAM expression and chemokines release, which eventually prevent migration of leukocytes. ${ }^{326,327}$ Glucocorticoids, cited as NF- $\kappa$ B inhibitors mediate the effects through inhibition of NF- $\kappa$ B transcriptional activity leading to suppression of proinflammatory genes. ${ }^{328}$ Taken together, IL-1 $\beta$ mediates: I) $\beta 2$ integrin dependent neutrophil migration and II) induction of NF- $\kappa \mathrm{B}$ dependent pro-inflammatory genes. In order to reduce both neutrophil migration and inflammation associated with IL-1 $\beta$ stimulation, we used MPS encapsulated and anti-ICAM-1 antibody conjugated liposomes (MPS-L ${ }^{\mathrm{Ab}}$ ). In the present study, liposomes were chosen for their flexibility: I) to anchor anti-ICAM-1 Ab onto the surface of liposomes for targeting to $\beta 2$ integrin receptors located on alveolar epithelium and II) to encapsulate and deliver MPS to alveolar endothelial cells for inhibition of pro-inflammatory cytokines (e.g. TNF- $\alpha$ and IL-6), chemokines (e.g. MCP-1) and CAMs (e.g. ICAM-1) expression.

To determine the effects of the targeting system in vivo, we used IL- $1 \beta$ induced lung inflammation rat model. MPS- $\mathrm{L}^{\mathrm{Ab}}$ suspension was administered by intravenous route prior to intra-tracheal (i.t.) instillation of IL-1 $\beta$ in male Sprague-Dawley rats. In vivo results demonstrated that MPS- $\mathrm{L}^{\mathrm{Ab}}$ treatment show a decrease in neutrophils migration and pro-inflammatory mediators' expression compared to IL-1 $\beta$ instilled (i.t.) positive control. The results of this study present evidence that MPS-L ${ }^{\mathrm{Ab}}$ reduces neutrophil migration by blocking $\beta 2$ integrin receptors; while MPS release from MPS-L ${ }^{\mathrm{Ab}}$ exhibited mild reduction in pulmonary edema.

\subsection{Experimental Section}

\subsubsection{Materials}

HSPC, DSPE-PEG 2000 , DSPE-PEG 2000 -maleimide were obtained from Avanti Polar Lipids (Birmingham, AL). MPS and recombinant human interleukin-1 $\beta$ were purchased from Sigma (St.Louis, MO). Antibodies obtained include: Anti- $\alpha$ v (P2W7; sc-9969;

Santa Cruz Biotechnology Inc., Santa Cruz, CA), anti- $\beta 6$ (H-110; sc-15329; Santa Cruz Biotechnology Inc), anti- $\beta 1$ (mAb13, Becton-Dickinson, Bedford, MA), anti- $\alpha \mathrm{v} \beta 3$ (LM609; Chemicon, Temecula, CA), anti- $\alpha v \beta 5$ (P1F6; Chemicon) and IgG (Chemicon). Gel electrophoresis chemicals were obtained from Bio-Rad Inc. (Hercules, CA). All other chemicals of ACS grade or higher were purchased either from Sigma (St.Louis, MO) or Fisher Scientific (Suwannee, GA). 


\subsubsection{Methods}

\subsubsection{Preparation of Liposomes}

MPS encapsulation into liposomes was achieved by transmembrane calcium acetate gradient method described in Chapter 4. Liposomes were prepared with a lipid composition of HSPC, CHOL, DSPE-PEG 2000 and DSPE-PEG ${ }_{2000}$-maleimide in 50: 45: 3: 2 molar ratios. Lipid (30 $\mathrm{mM}$ total phospholipids) solution in chloroform-methanol solution $(3: 1 \% \mathrm{v} / \mathrm{v})$ was subjected to vacuum drying at $40^{\circ} \mathrm{C}$ to form a thin dry film, which was hydrated with calcium acetate buffer $(200 \mathrm{mM})$ at $65^{\circ} \mathrm{C}$ to form MLVs. These vesicles were extruded through $200 / 100 \mathrm{~nm}$ sandwiched polycarbonate membrane filters using LIPEX ${ }^{\mathrm{TM}}$ extruder. The MLVs were subjected to 6 extrusion passes to obtain liposomes with a mean diameter of 100-120 nm.

To create a transmembrane calcium acetate gradient across the liposomes, the extraliposomal calcium acetate buffer was replaced with $0.9 \%$ saline solution by dialysis (10,000 Da MWCO). Followed by generation of calcium acetate gradient, liposomes were mixed and incubated with MPS solution [19 $\mathrm{mM}$ ] at $48^{\circ} \mathrm{C}$ for $6 \mathrm{~min}$ and then cooled to $4^{\circ} \mathrm{C}$. Non-encapsulated MPS and any traces of calcium acetate were removed by dialysis of MPS-L at $4^{\circ} \mathrm{C}$ for $6 \mathrm{~h}$.

As shown in Figure 7.1, anti-ICAM-1 anti-body solution $(1 \mathrm{mg} / \mathrm{mL})$ was incubated with mercapto-ethylamine (MEA; $3 \mathrm{mg} / \mathrm{mL}, \mathrm{pH} 6.0$ ) at $30^{\circ} \mathrm{C}$ for $20 \mathrm{~min}$. Followed by incubation; the anti-body solution was de-salted to remove MEA using de-salted columns. The de-salting process was repeated 3 times to remove any traces of MEA. Now, the anti-body fragments were subjected to fractionation to isolate anti-body fragments. Fraction number, 6-8 obtained mFAb'2 fragments and fractions, 16-17 obtained $\mathrm{mFab}$ fragments. The isolated $\mathrm{mFab}$ ' fragments were used for conjugation with $\mathrm{DSPE}_{-\mathrm{PEG}} \mathrm{200}$-maleimide in $500 \mu \mathrm{L}$ of HEPES buffer, $\mathrm{pH} 6.8$ for $2 \mathrm{~h}$ at $30^{\circ} \mathrm{C}$, under a nitrogen atmosphere. The reaction was stopped with N-ethyl maleimide solution $(8 \mathrm{mM})$. Now, the lipid-mFab' conjugate was dialyzed for $6 \mathrm{~h}$ at $4^{\circ} \mathrm{C}$ to remove $\mathrm{N}$-ethyl maleimide. The amount of $\mathrm{mFab}$ ' coupled to DSPE-PEG 2000 -maleimide was estimated qualitatively by SDS-PAGE and quantitatively by using Bio-Rad protein assay kit. Approximately, 27\% (molar) yield was obtained.

\subsubsection{SDS-PAGE}

Immunoliposomes were run SDS-PAGE under non-reducing conditions to avoid further fragmentation of anti-body. In this process, reducing agents were not added during the preparation of both samples. As a result, the resolution of the immunoliposomes is based on both molecular weight and charge resided on the surface of liposomes (Figure 7.2). 


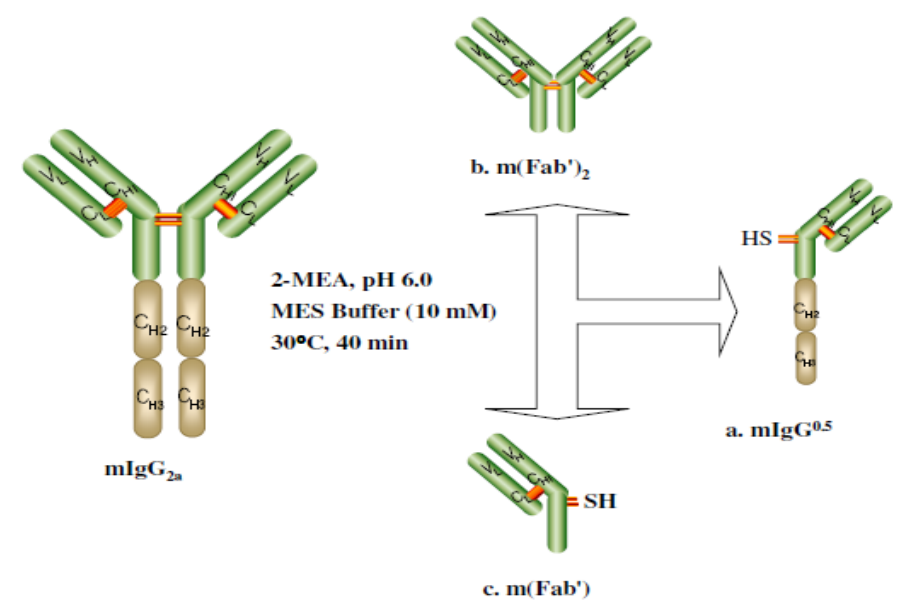

A. Fragmentation of IgG Antibody

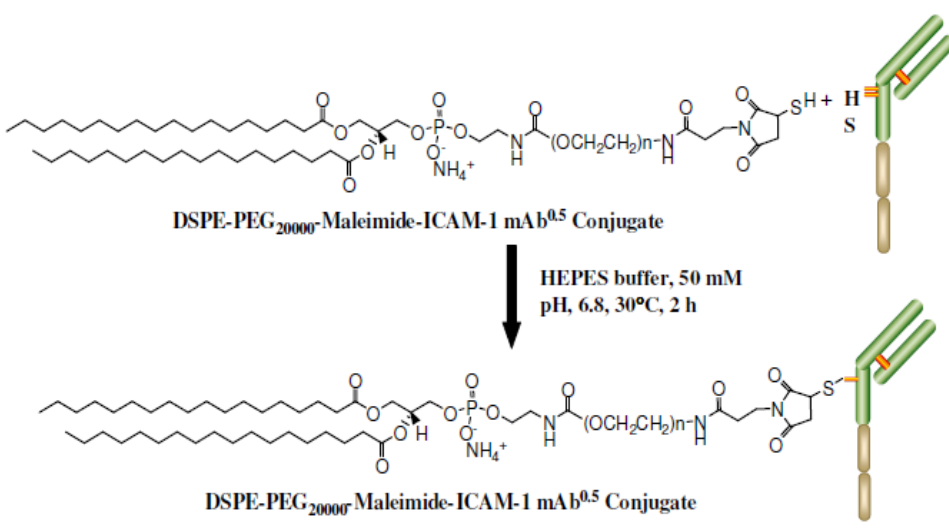

B. Coupling of mFab' with DSPE-PEG2000-maleimide

Figure 7.1 Schematic representation of the coupling of anti-ICAM-1 antibody fragment tethered to the surface of liposomes.

In step A, antibody fragments were synthesized by using MEA buffer. In step B, following the cleavage of antibody, antibody fragments were coupled to DSPE-PEG $2000^{-}$ maleimide by Michael addition reaction. 


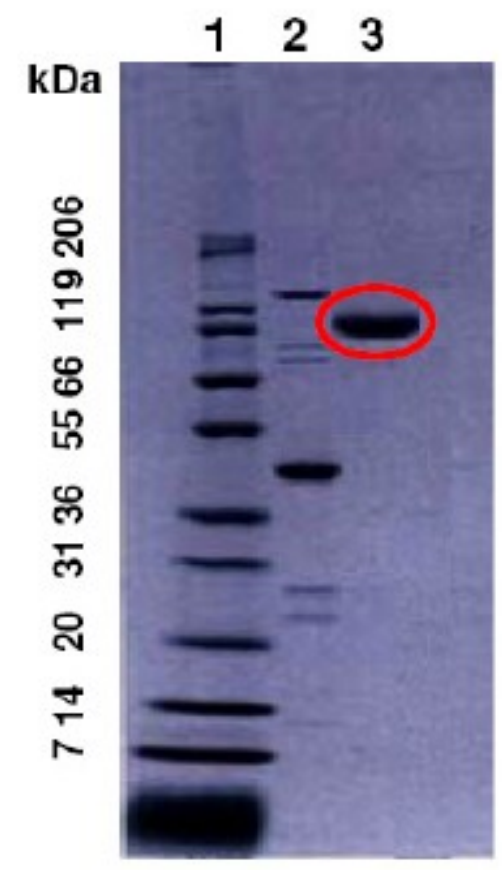

Figure 7.2 SDS-PAGE analysis of DSPE-PEG $2000-\mathrm{mFab}^{\prime}$ fragment under nonreducing conditions.

The molecular weight heavy chain is $50,000 \mathrm{Da}$ and that of light chain is 23,000 Da. And the molecular weight of the lipid is 20,000 Da. Total molecular weight of the conjugate is 93,000 Da which is red circled on lane 3. The bands on lane 1 correspond to standard molecular weight markers (high molecular weight mixture; Bio-Rad). The bands on lane 2 correspond to light chain and heavy chains. 


\subsubsection{Insertion of Lipid-antibody Conjugates into Lipid Bilayers of Liposomes}

Lipid-antibody was inserted into the bilayers of liposomes by post-insertion method as described in Chapter 4.

\subsubsection{Particle Colloidal Properties}

Mean diameter and zeta potential of liposomes were evaluated by DLS studies as described elsewhere.

\subsubsection{Animals}

Male Sprague-Dawley rats were used in all experiments. The experimental protocols were approved by IACUC of the University of Tennessee, Memphis.

\subsubsection{Systemic Administration of Liposome Formulations}

At least 30 min prior to IL-1 $\beta$ (i.t.) instillation, rats were administered with liposome (PL or MPS-L or MPS-L ${ }^{\mathrm{Ab}}$ ) formulations at $2 \mathrm{mg} / \mathrm{kg}$. Dose selection was based on initial dose escalation studies to see the pharmacodynamic effects. Twenty-four hours after the treatment, animals from all groups were sacrificed and lung tissue samples from each animal were fixed in $10 \% \mathrm{v} / \mathrm{v}$ buffered formalin.

\subsubsection{IL-1 $\beta$ Instillation and BALF Collection}

Male Sprague-Dawley rats, weighing 125-150 g were used in the study. Rats were fed normal diet before the experiment with free access to water. Following anesthesia (ketamine/xylazine, $87 / 13 \mathrm{mg} / \mathrm{kg}$ ), a midline tracheal incision was made to expose the trachea. A 26 gauge needle was advanced endotracheally to instill IL-1 $\beta$ solution (200 $\mu \mathrm{L}, 100 \mu \mathrm{g} / \mathrm{mL}$ in PBS, $\mathrm{pH}$ 7.2) or PBS alone (control). Following the IL-1 $\beta$ instillation, tracheal opening was sutured and animals were returned to cage.

Twenty four hours post IL-1 $\beta$ administration, the lung vascular bed was flushed twice by injecting $10 \mathrm{~mL}$ ice-cold PBS into right ventricle. Followed by overdose of pentobarbital $(100 \mathrm{mg} / \mathrm{kg}$, i.v), tracheal sutures were cut open to expose trachea. BALF collection was performed by inserting a 26 gauge needle into trachea and lungs were lavaged twice with PBS $(3 \mathrm{~mL})$. BALF was collected into cryovials and centrifuged at $1100 \mathrm{xg}, 4^{\circ} \mathrm{C}$ for 10 min. The supernatant was stored at $-70^{\circ} \mathrm{C}$ for cytokines and chemokines analysis and the cell pellet was subjected to neutrophils estimation. Aliquots of BALF were estimated for protein concentration by UV absorbance at $280 \mathrm{~nm}$ using albumin (Bio-Rad) as standard. Also, post IL-1 $\beta$ administration, blood was collected at different time intervals for estimation of MPS levels. 


\subsubsection{Estimation of BALF Cytokines and Chemokines}

BALF supernatant collected and stored as described above was analyzed for cytokines (TNF- $\alpha$ ) by ELISA method according to manufacturer (R\&D Systems, Minneapolis, $\mathrm{MN}$ ) instructions.

\subsubsection{Estimation of Lung Neutrophil Content}

Neutrophil content was assayed as described previously. ${ }^{329}$

\subsubsection{Histological Examination of Lungs}

Histological examination was performed as described in Chapter 6.

\subsubsection{Statistical Analysis}

All data reported are arithmetic means. Error bars represent one SEM Differences between means of indicated groups were analyzed by ANOVA with Bonferroni corrections for multiple comparisons. A P value $<0.05$ was considered to be statistically significant.

\subsection{Results and Discussion}

\subsubsection{Physico-chemical Characterization of MPS-L ${ }^{\mathrm{Ab}}$}

The colloidal properties of MPS-L ${ }^{\mathrm{Ab}}$ and MPS-L determined by DLS method were stable over 14 days of shelf life. As shown in Table.7.1, mean particle size and polydispersity index of MPS- $\mathrm{L}^{\mathrm{Ab}}$ is large than MPS-L. This could be attributed due to the large molecular weight of the ligand on the surface of liposomes. Zeta potential of MPS-L ${ }^{\mathrm{Ab}}$ was lower than MPS-L due to a large content of positive charged amino acids residing on surface of the antibody.

\subsubsection{Effect of MPS- $\mathrm{L}^{\mathrm{Ab}}$ on BALF Protein Concentration in IL- $1 \beta$ Induced Rats}

Quantification of BALF protein concentration is considered as an indicator of increase in lung vascular and epithelial permeability resulting from inflammatory injury. In the present study, BALF protein concentration was quantified in control and treated groups. As represented in Figure 7.3, protein concentration in the BALF of IL-1 $\beta$ (i.t.) instilled group was nearly 2.75 folds that of rats receiving saline (i.t.) (IL- $1 \beta$ group, $0.52 \pm 0.03$ $\mathrm{mg} / \mathrm{mL}$; saline group, $0.18 \pm 0.02 \mathrm{mg} / \mathrm{mL} ; \mathrm{p}<0.001)$. A significant inhibition of BALF 
Table 7.1 Physico-chemical properties of MPS- $\mathrm{L}^{\mathrm{Ab}}$ used in in vivo studies.

\begin{tabular}{lccc}
\hline Liposomes & Mean diameter, Zavg $(\mathrm{nm})$ & Zeta potential, $\zeta(\mathrm{mV})$ & $\begin{array}{c}\text { MPS incorporation } \\
\text { efficiency }(\% \mathrm{w} / \mathrm{v})\end{array}$ \\
\hline PL & $113 \pm 5$ & $-22.7 \pm 2.8$ & NA \\
MPS-L & $110 \pm 18$ & $-20.4 \pm 1.4$ & $\cong 59$ \\
MPS-L $^{\mathrm{Ab}}$ & $135 \pm 14$ & $-9.1 \pm 3.7$ & $\cong 24$ \\
\hline
\end{tabular}

Each value represents the mean $\pm \operatorname{SEM}(n=3)$. Mean diameter and zeta potential of liposome formulations were determined at $25^{\circ} \mathrm{C}$. NA indicates not applied. NA was applied to the liposome formulations devoid of MPS encapsulation.

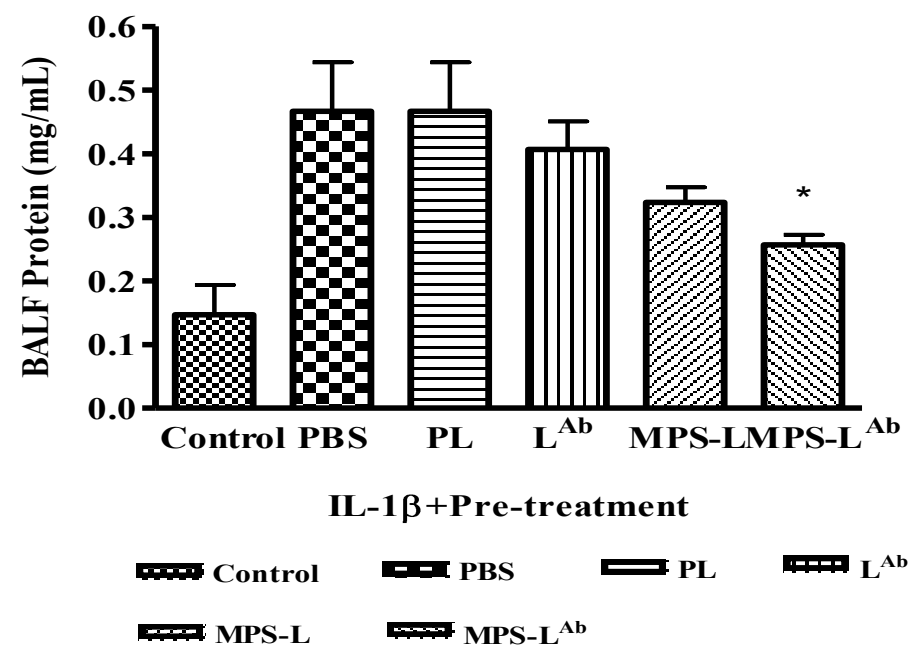

Figure 7.3 Histogram of inhibition of BALF protein in male Sprague-Dawley rats after systemic administration of MPS-L ${ }^{\mathrm{Ab}}$.

BALF protein levels were measured by Bio-Rad micro-protein assay. Results are expressed as mean \pm SEM of 3 samples. Statistically significant differences $(* \mathrm{P}<0.05)$ compared with IL-1 $\beta$ treatment alone in each experiment. 
protein concentration was observed in rats pretreated with MPS-L ${ }^{\mathrm{Ab}}(0.26 \pm 0.01 \mathrm{mg} / \mathrm{mL}$; $\mathrm{p}<0.05$ ) compared to IL-1 $\beta$ (i.t.) instilled group. To delineate the effects of MPS and $\mathrm{L}^{\mathrm{Ab}}$ on the inhibition of BALF protein, rats were pretreated with MPS-L or L ${ }^{\mathrm{Ab}}$, respectively. A mild reduction of BALF protein was observed in rats pretreated with MPS-L $(0.32 \pm 0.02 \mathrm{mg} / \mathrm{mL} ; \mathrm{P}>0.05)$ and $\mathrm{L}^{\mathrm{Ab}}(0.44 \pm 0.02 \mathrm{mg} / \mathrm{mL} ; \mathrm{P}>0.05)$, which indicate formulations had no effect on alveolar endothelial permeability. The data suggests that protein permeability is mediated by pathways other than $\beta 2$ integrins. The inhibition of protein permeability observed with MPS-L ${ }^{\mathrm{Ab}}$ could be due to the synergistic effect of MPS delivery and anti-ICAM-1 Ab targeting.

\subsubsection{Effect of MPS-L ${ }^{\mathrm{Ab}}$ on MCP-1 Production in BALF of IL-1 $\beta$ Induced Rats}

BALF levels at $24 \mathrm{~h}$ following IL-1 $\beta$ (i.t.) instillation in pretreated (MPS-L ${ }^{\mathrm{Ab}}$, MPS-L or $L^{\mathrm{Ab}}$ ) rat groups were measured. Saline and IL-1 $\beta$ alone instilled groups were treated as negative and positive control groups. As shown in Figure 7.4, MPS-L ${ }^{\mathrm{Ab}}$ treatment resulted in a decrease of MCP-1 (1499.2 $\pm 85.2 \mathrm{pg} / \mathrm{mL}$ in IL-1 $\beta$ alone instilled rats vs. $540.7 \pm 61.2 \mathrm{pg} / \mathrm{mL}$ in MPS- $\mathrm{L}^{\mathrm{Ab}}$ treated rats, $\left.\mathrm{P}<0.05\right)$. MPS-L also exerted the similar effects of MCP-1 inhibition as MPS- $\mathrm{L}^{\mathrm{Ab}}$. $\mathrm{L}^{\mathrm{Ab}}$ has no influence on MCP-1 production, whose levels were similar to that of IL-1 $\beta$ instilled positive control group.

\subsubsection{Effect of MPS-L ${ }^{\mathrm{Ab}}$ on TNF- $\alpha$ Production in BALF of IL-1 $\beta$ Induced Rats}

As compared with saline-treated controls, IL-1 $\beta$ (i.t.) instillation induced a significant increase in BALF levels of TNF- $\alpha$ (Figure 7.5). After MPS-L ${ }^{\mathrm{Ab}}$ and MPLS-L treatment, BALF levels of TNF- $\alpha$ were significantly $(\mathrm{P}<0.05)$ lower. In contrast, BALF levels of TNF- $\alpha$ in animals pretreated with $\mathrm{L}^{\mathrm{Ab}}$ had no effect on TNF- $\alpha$ production.

\subsubsection{Effect of MPS- $\mathrm{L}^{\mathrm{Ab}}$ on Neutrophil Migration of IL-1 $\beta$ Induced Rats}

For quantitative assessment of neutrophil migration into alveolar spaces, MPO assay of BALF was performed in pretreated rat groups (MPS-L ${ }^{\mathrm{Ab}}$, MPS-L or $\mathrm{L}^{\mathrm{Ab}}$ ) post IL-1 $\beta$ (i.t.) instillation. As illustrated in Figure 7.6, pretreatment of rats with $\mathrm{L}^{\mathrm{Ab}}$ inhibited the neutrophil migration into alveolar spaces of rats receiving IL-1 $\beta$ (i.t.) as compared with saline treated rats $\left(1.2 \pm 0.2 \times 10^{6} ; \mathrm{p}<0.05\right.$ in $\mathrm{L}^{\mathrm{Ab}}$ treated group vs. $2.4 \pm 0.5 \times 10^{6} ; \mathrm{P}<0.05$ in saline treated group). Inhibition of neutrophil migration was similar to $\mathrm{L}^{\mathrm{Ab}}$ pretreatment. Only, a mild reduction of BALF neutrophil content was observed with MPS-L.

\subsubsection{Effect of MPS- $\mathrm{L}^{\mathrm{Ab}}$ on Neutrophil Accumulation in Lung Parenchyma}

Rats received IL-1 $\beta$ (i.t.) or saline as a control, and were sacrificed $24 \mathrm{~h}$ later. As shown in Figure 7.7, rats receiving IL-1 $\beta$ showed a 11.3 fold increase $(\mathrm{P}<0.001)$ in lung 


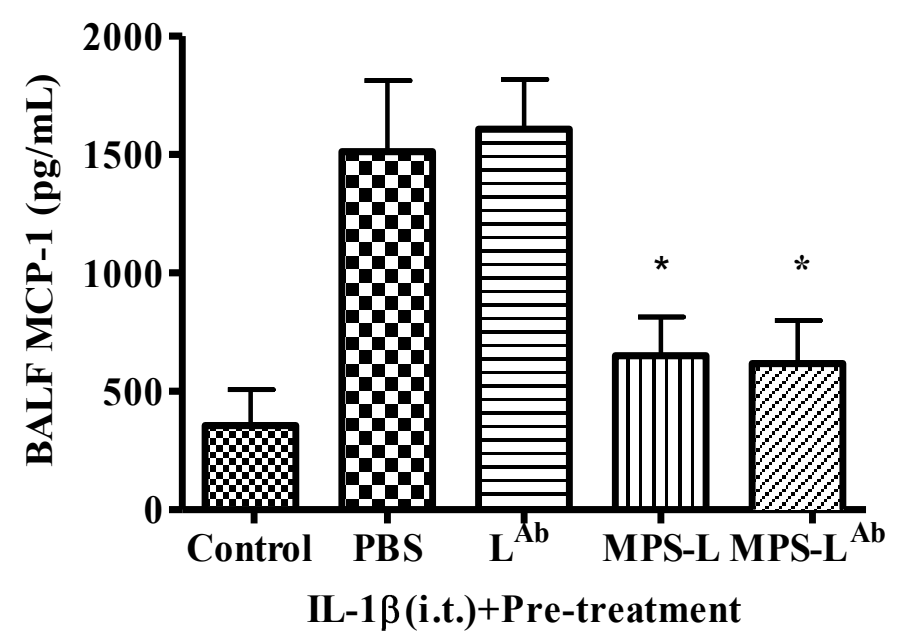

Figure 7.4 Histogram of inhibition of BALF MCP-1 levels in male Sprague-Dawley rats after systemic administration of MPS-L ${ }^{\mathrm{Ab}}$.

Results are expressed as a mean \pm SEM of 3 samples. Statistically significant differences $(* \mathrm{P}<0.05)$ compared with IL-1 $\beta$ treatment alone in each experiment.

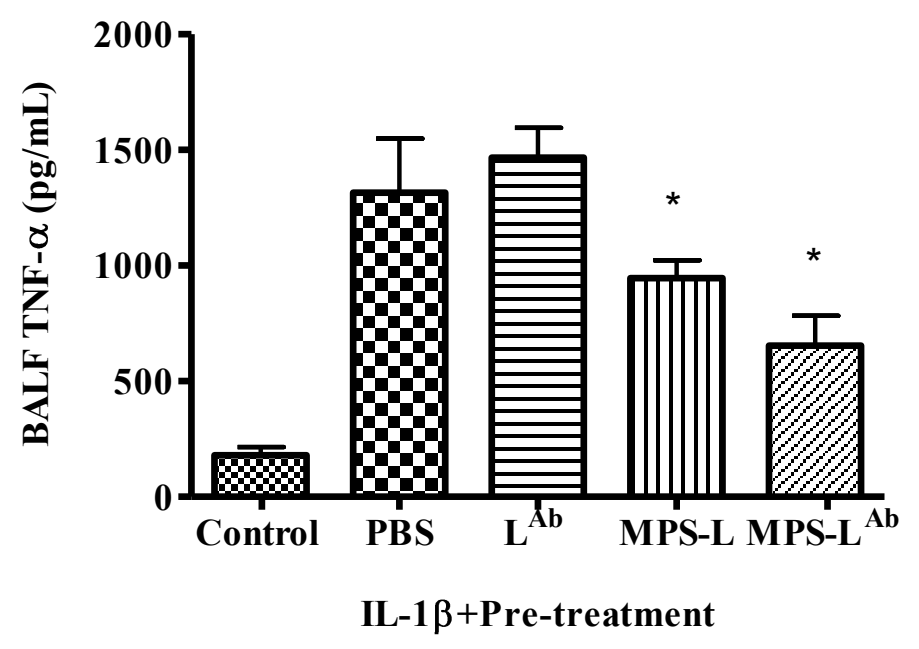

Figure 7.5 Histogram of inhibition of BALF TNF- $\alpha$ levels in male Sprague-Dawley rats after systemic administration of MPS-L ${ }^{\mathrm{Ab}}$.

TNF- $\alpha$ levels were measured by ELISA. Results are expressed as a mean \pm SEM of 3 samples. Statistically significant differences $(* \mathrm{P}<0.05)$ compared with IL- $1 \beta$ treatment alone in each experiment. 


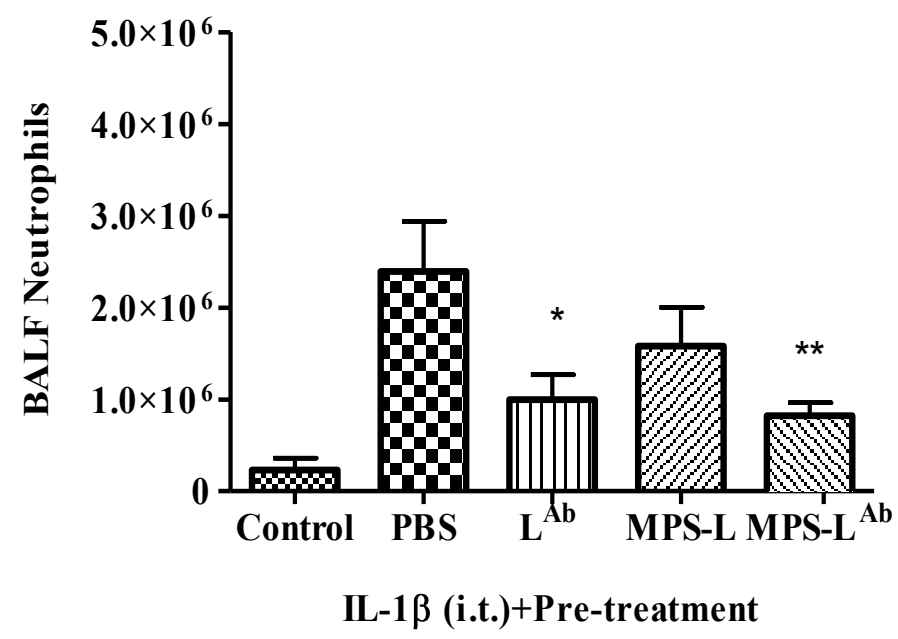

Figure 7.6 Histogram of inhibition of BALF levels of neutrophils after nebulization of MPS- $\mathrm{L}^{\mathrm{Ab}}$ in male Spague-Dawley rats.

Neutrophil levels were analyzed by Histopaque and MPO assays. Results are expressed as a mean \pm SEM of 3 samples. Statistically significant differences $(* \mathrm{P}<0.05, * * \mathrm{P}<0.01)$ compared with IL-1 $\beta$ treatment alone in each experiment. 


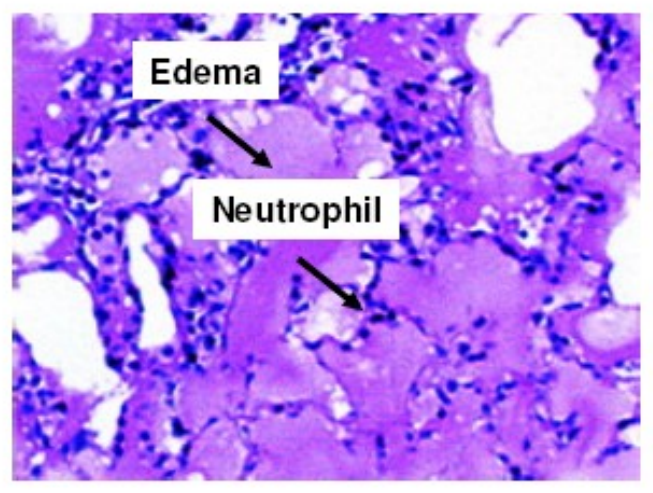

IL-1 $\beta$

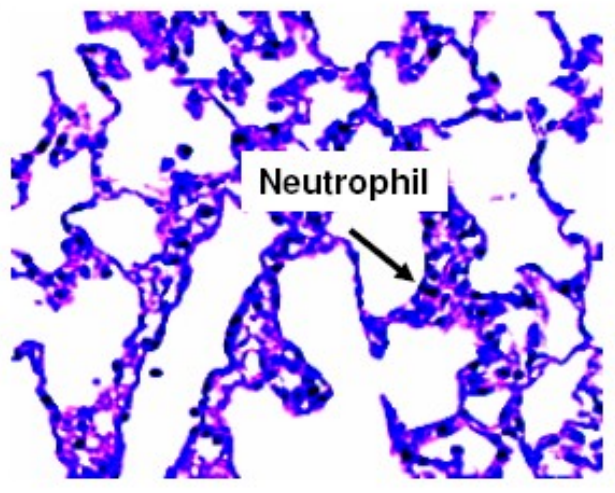

$L^{A b}$

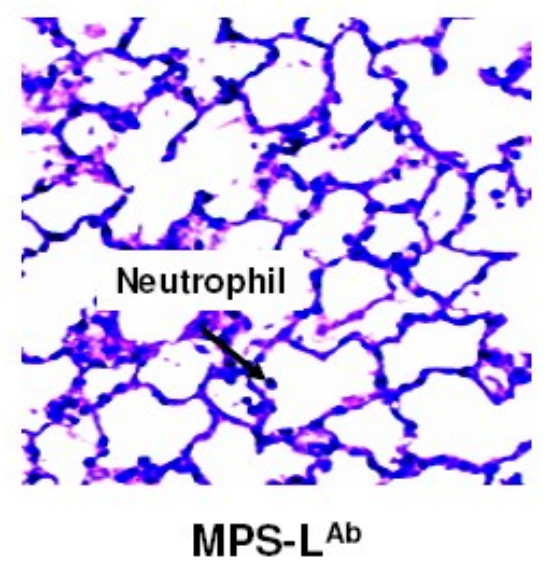

Figure 7.7 Histological sections of lung parenchyma after haematoxylin treatment to stain neutrophils.

Lung parenchyma sections represent the edema and neutrophil modulation pattern after MPS- ${ }^{\mathrm{Ab}}$ pretreatment. IL-1 $\beta$ instilled lung sections shows profuse edema and neutrophil migration. Both MPS-L ${ }^{\mathrm{Ab}}$ and $\mathrm{L}^{\mathrm{Ab}}$ showed neutrophil reduction in alveoli. However, MPS- $\mathrm{L}^{\mathrm{Ab}}$ significantly reduced edema also. All the sections are representative of $\mathrm{n}=3$ samples. 
parenchyma neutrophil content to $3.2 \pm 0.8 \times 10^{8}$ over saline control. To investigate the effect of MPS-L ${ }^{\mathrm{Ab}}$ on neutrophil migration into the lung during ALI, neutrophil accumulation which occurs prior to migration into alveoli was estimated by MPO assay of the lung tissue. Besides, haematoxylin staining technique was adopted to visualize the neutrophil accumulation in paraffin embedded lung parenchyma sections. From the staining, it is evident that MPS- $\mathrm{L}^{\mathrm{Ab}}(\mathrm{P}<0.01)$ completely attenuated neutrophil accumulation compared to saline control. Also, $\mathrm{L}^{\mathrm{Ab}}(\mathrm{P}<0.05)$ exerted a significant inhibition on lung parenchymal accumulation, which runs in correlation with BALF neutrophil content.

\subsection{Summary}

In summary, these studies demonstrate that $\beta 2$ integrins mediate the neutrophil migration into alveoli in response to IL-1 $\beta$ stimulation. To disrupt the interaction between $\beta 2$ integrins and ICAM-1 receptors of alveolar endothelium, anti-ICAM-1 antibody conjugated liposomes were administered by systemic route. This strategy was adopted as ICAM-1 receptors could be accessed from the circulation rather than from the alveoli. MPS- $\mathrm{L}^{\mathrm{Ab}}$ completely attenuated neutrophil migration. However, only a mild reduction in pulmonary edema and inhibition on inflammatory response were observed. This could possibly be due to the fact that IL-1 $\beta$ stimulates $\alpha v \beta 5$ and $\alpha v \beta 6$ integrins and mediates pulmonary edema. Anti-ICAM-1 antibody is not specific to either of the integrins and hence could exert no inhibitory effects on integrin receptors. In such cases, multiple ligand targeting strategy or combination approaches using integrin and ICAM-1 receptor specific targeting agents may favor the inhibition of pulmonary edema and neutrophil migration. 


\section{CHAPTER 8. SUMMARY AND CONCLUSIONS}

The alveolar epithelium and endothelium are prime targets for intervention of lung inflammation. ALI inflammation is characterized by pulmonary edema, pro-inflammatory inflammatory mediators' production and leukocyte migration into alveoli. Underlying the inflammation, integrin receptors play a critical role in the mediation of various pathological events of ALI. Proinflammatory cytokines like TNF- $\alpha$ and IL- $1 \beta$ activate alveolar epithelium and endothelium via NF- $\kappa \mathrm{B}$ pathway. The activated $\mathrm{EC}$ excrete cytokines and chemokines, which in concert with cellular adhesion molecules promote leukocyte recruitment and migration. Blockade of these processes has attained an immense attention in recent years, and many new signal transduction inhibitors against $\mathrm{NF}-\kappa \mathrm{B}$ were developed.

In this thesis, I developed novel surface modified liposomes aiming at the inhibition of proinflammatory stimuli in the lung. The first part of the thesis concerns the development and stabilization of surface modified nano-sized targeted liposomes. In the second part of the presented work, the drug targeting concept was applied to anti-inflammatory agents, in order to selectively deliver therapeutic moieties to the affected disease sites. I applied a cyclic arginine-glycine-D-aspartic acid tri-peptide and anti-ICAM-1 antibody as targeting ligands. The cRGD-peptide selectively binds to subsets of integrin receptor that are upregulated on activated epithelium and endothelium, but hardly expressed on quiescent cells. Anti-ICAM-1 antibody selectively binds to ICAM-1 receptors upregulated on alveolar endothelium. The task of a carrier is to carry the drug to the disease site. For this purpose, natural and synthetic polymers, antibodies (ligand and carrier), nanoparticles and even erythrocytes have been used. I chose liposomes for it is flexible enough for drug encapsulation, and imparts long circulation and targeting properties. Using functionalized lipids, I modified the surface of liposomes with cRGD-peptide and anti-ICAM-1 Ab, leading to a receptor binding drug carriers with high avidity.

However, liposomes are thermodynamically unstable structures and undergo aggregation/fusion resulting in the loss of colloidal properties of the drug delivery systems. I addressed the concerns related to the stability of these uniquely designed targeted drug delivery systems. Lyophilization approach was adopted to improve the shelf-life of the liposomes. However, lyophilization itself is an aggressive process, which may be detrimental to the liposomes. Disaccharides and aminoacids were investigated to exert lyoprotection on these colloidal systems. Our studies showed that trehalose and sucrose among the sugars, and lysine among the aminoacids protected the liposomes from aggregation and leakage of the encapsulated drug moieties.

This thesis reports how improved the delivery of the drug to the targeted sites with cRGD-peptide and anti-ICAM-1 Ab conjugated systems in comparison to the nontargeted systems. The drug targeting systems showed high cell internalization and adequate drug release, since the uptake eventually led to release of the drug. To enhance overall delivery of the targeted liposomes, I explored different routes of administration to take advantage of the spatial and temporal expression of the receptors. Nebulization and 
systemic routes of administration were investigated. Since $\alpha v \beta 6$ integrins are expressed on the apical side of the alveolar epithelium, I adopted nebulization approach for targeting to the integrin receptors. Nebulization of MPS- $\mathrm{L}^{\mathrm{CRGD}}$ was performed prior to IL$1 \beta$ instillation in rats. MPS- $\mathrm{L}^{\mathrm{CRGD}}$ liposome drug targeting system inhibited the inflammation and neutrophil migration associated with IL-1 $\beta$ instilled ALI model. The inhibition of $\alpha v \beta 6$ integrins blocked the IL- $1 \beta$ mediated protein permeability across the distal lung epithelium, and prevented, in large part, the development of pulmonary edema. MPS released from the MPS-L ${ }^{\mathrm{CRGD}}$ modulated the NF- $\mathrm{\kappa B}$ transcription, and attenuated the production of pro-inflammatory TNF- $\alpha$ and MCP-1 levels. Neutrophil migration and accumulation in lungs were inhibited. This study demonstrated that route of administration and targeted delivery system potentiate anti-inflammatory efficacy of MPS-L ${ }^{\mathrm{CRGD}}$ targeted delivery system.

I demonstrated that MPS- $\mathrm{L}^{\mathrm{Ab}}$ could be targeted to ICAM-1 receptors, and thereby reduce the neutrophil migration into alveoli. MPS- $\mathrm{L}^{\mathrm{Ab}}$ was able to inhibit the pro-inflammatory cytokines and chemokines production. However, only mild effects on edema were observed.

In conclusion, I developed receptor specific surface modified nanoparticles to lung in an attempt to inhibit ALI pathogenesis. I demonstrated that route of administration of the delivery system plays a critical role to exert beneficial effects on ALI treatment. Furthermore, I stabilized the targeted liposomes, whose shelf-life was improved. The research works strengthened the development of novel nanoparticulate systems, in particular, liposomes for achieving anti-inflammatory drugs with high efficiency and specificity. Our studies demonstrated that the demands of many of these listed parameters were met, but the mild outcome for MPS- $\mathrm{L}^{\mathrm{Ab}}$ demands that further work is required to see the benefits. Nevertheless, our drug targeting approach must be transferred to new drug moieties. Thereby, we can exploit our experience on targeting surface modified liposomes as shown in this thesis. The novel drug encapsulation and conjugation strategies, along with the strategic delivery facilitated improved results. 


\section{LIST OF REFERENCES}

1. Ashbaugh, D.G., Bigelow, D.B., Petty, T.L. \& Levine, B.E. Acute respiratory distress in adults. Lancet 2, 319-323 (1967).

2. Bernard, G.R. et al. The American-European Consensus Conference on ARDS. Definitions, mechanisms, relevant outcomes, and clinical trial coordination. Am J Respir Crit Care Med 149, 818-824 (1994).

3. Morris, A.H. et al. Randomized clinical trial of pressure-controlled inverse ratio ventilation and extracorporeal $\mathrm{CO}_{2}$ removal for adult respiratory distress syndrome. Am J Respir Crit Care Med 149, 295-305 (1994).

4. Ware, L.B. \& Matthay, M.A. The acute respiratory distress syndrome. $N$ Engl $J$ Med 342, 1334-1349 (2000).

5. Davidson, T.A., Caldwell, E.S., Curtis, J.R., Hudson, L.D. \& Steinberg, K.P. Reduced quality of life in survivors of acute respiratory distress syndrome compared with critically ill control patients. JAMA 281, 354-360 (1999).

6. Atabai, K. \& Matthay, M.A. The pulmonary physician in critical care. 5: Acute lung injury and the acute respiratory distress syndrome: Definitions and epidemiology. Thorax 57, 452-458 (2002).

7. Fowler, A.A. et al. Adult respiratory distress syndrome: Risk with common predispositions. Ann Intern Med 98, 593-597 (1983).

8. Dorinsky, P.M. \& Gadek, J.E. Multiple organ failure. Clin Chest Med 11, 581-591 (1990).

9. Welty-Wolf, K.E., Carraway, M.S., Ortel, T.L. \& Piantadosi, C.A. Coagulation and inflammation in acute lung injury. Thromb Haemost 88, 17-25 (2002).

10. Downey, G.R. Mechanism of acute lung injury. Curr Opin Pulm Med 3, 177-241 (1997).

11. Park, W.Y. et al. Cytokine balance in the lungs of patients with acute respiratory distress syndrome. Am J Respir Crit Care Med 164, 1896-1903 (2001).

12. Strieter, R.M., Kunkel, S.L., Keane, M.P. \& Standiford, T.J. Chemokines in lung injury: Thomas A. Neff lecture. Chest 116, 103S-110S (1999).

13. Martin, T.R. Lung cytokines and ARDS: Roger S. Mitchell lecture. Chest 116, 2S8S (1999).

14. Zimmerman, G.A. et al. Endothelial activation in ARDS. Chest 116, 18S-24S (1999).

15. Doerschuk, C., Mizgerd, J., Kubo, H., Qin, L. \& Kumasaka, T. Adhesion molecules and cellular biomechanical changes in acute lung injury: Giles F. Filley lecture. Chest 116, 37S-43S (1999).

16. Abraham, E. Neutrophils and acute lung injury. Crit Care Med 31, S195-199 (2003).

17. Downey, G.P., Dong, Q., Kruger, J., Dedhar, S. \& Cherapanov, V. Regulation of neutrophil activation in acute lung injury. Chest 116, 46S-54S (1999).

18. Pittet, J.F. et al. TGF-beta is a critical mediator of acute lung injury. J Clin Invest 107, 1537-1544 (2001). 
19. Munger, J.S. et al. The integrin alpha v beta 6 binds and activates latent TGF beta 1: A mechanism for regulating pulmonary inflammation and fibrosis. Cell 96, 319-328 (1999).

20. Jenkins, R.G. et al. Ligation of protease-activated receptor 1 enhances alpha(v)beta6 integrin-dependent TGF-beta activation and promotes acute lung injury. J Clin Invest 116, 1606-1614 (2006).

21. Ganter, M.T. et al. Interleukin-1 $\{$ beta $\}$ causes acute lung injury via $\{$ alpha $\}$ v $\{$ beta $\} 5$ and $\{$ alpha $\}$ v $\{$ beta 6 integrin-dependent mechanisms. Circ Res 102, 804-812 (2008).

22. Michel, C.C. The transport of albumin: a critique of the vesicular system in transendothelial transport. Am Rev Respir Dis 146, S32-36 (1992).

23. Garcia, J.G., Davis, H.W. \& Patterson, C.E. Regulation of endothelial cell gap formation and barrier dysfunction: role of myosin light chain phosphorylation. $J$ Cell Physiol 163, 510-522 (1995).

24. Lum, H. \& Malik, A.B. Regulation of vascular endothelial barrier function. Am J Physiol Lung Cell Mol Physiol 267, L223-241 (1994).

25. Lu, Q. et al. Transforming growth factor-betal-induced endothelial barrier dysfunction involves Smad2-dependent p38 activation and subsequent RhoA activation. J Appl Physiol 101, 375-384 (2006).

26. Sun, H., Breslin, J.W., Zhu, J., Yuan, S.Y. \& Wu, M.H. Rho and ROCK signaling in VEGF-induced microvascular endothelial hyperpermeability. Microcirculation 13, 237-247 (2006).

27. Clements, R.T., Minnear, F.L., Singer, H.A., Keller, R.S. \& Vincent, P.A. RhoA and Rho-kinase dependent and independent signals mediate TGF-beta-induced pulmonary endothelial cytoskeletal reorganization and permeability. Am J Physiol Lung Cell Mol Physiol 288, L294-306 (2005).

28. von Andrian, U.H. et al. Two-step model of leukocyte-endothelial cell interaction in inflammation: distinct roles for LECAM-1 and the leukocyte beta 2 integrins in vivo. Proc Natl Acad Sci U S A 88, 7538-7542 (1991).

29. Lawrence, M.B. \& Springer, T.A. Leukocytes roll on a selectin at physiologic flow rates: distinction from and prerequisite for adhesion through integrins. Cell 65, 859-873 (1991).

30. Li, X., Abdi, K., Rawn, J., Mackay, C.R. \& Mentzer, S.J. LFA-1 and L-selectin regulation of recirculating lymphocyte tethering and rolling on lung microvascular endothelium. Am J Respir Cell Mol Biol 14, 398-406 (1996).

31. Dransfield, I., Cabanas, C., Barrett, J. \& Hogg, N. Interaction of leukocyte integrins with ligand is necessary but not sufficient for function. J Cell Biol 116, 1527-1535 (1992).

32. Springer, T.A. Traffic signals for lymphocyte recirculation and leukocyte emigration: the multistep paradigm. Cell 76, 301-314 (1994).

33. Doerschuk, C.M., Beyers, N., Coxson, H.O., Wiggs, B. \& Hogg, J.C. Comparison of neutrophil and capillary diameters and their relation to neutrophil sequestration in the lung. J Appl Physiol 74, 3040-3045 (1993).

34. Kuebler, W.M., Kuhnle, G.E., Groh, J. \& Goetz, A.E. Contribution of selectins to leucocyte sequestration in pulmonary microvessels by intravital microscopy in rabbits. J Physiol 501 ( Pt 2), 375-386 (1997). 
35. Bevilacqua, M.P. \& Nelson, R.M. Selectins. J Clin Invest 91, 379-387 (1993).

36. Crockett-Torabi, E. \& Fantone, J.C. The selectins: insights into selectin-induced intracellular signaling in leukocytes. Immunol Res 14, 237-251 (1995).

37. Simon, S.I. et al. L-selectin (CD62L) cross-linking signals neutrophil adhesive functions via the Mac-1 (CD11b/CD18) beta2-integrin. J Immunol 155, 15021514 (1995).

38. Zimmerman, G.A., McIntyre, T.M. \& Prescott, S.M. Adhesion and signaling in vascular cell--cell interactions. J Clin Invest 98, 1699-1702 (1996).

39. Marchesi, V. \& Florey, H. Electron micrographic observations on the emigration of leucocytes. J Exp Physiol 45, 343-348 (1960).

40. Zen, K. \& Parkos, C. Leukocyte-epithelial interactions. Curr Opin Cell Biol 15, 557-564 (2003).

41. Liu, Y. et al. Regulation of leukocyte transmigration: cell surface interactions and signaling events. J Immunol 172, 7-13 (2004).

42. Doerschuk, C.M., Winn, R.K., Coxson, H.O. \& Harlan, J.M. CD18-dependent and - independent mechanisms of neutrophil emigration in the pulmonary and systemic microcirculation of rabbits. J Immunol 144, 2327-2333 (1990).

43. Qin, L. et al. The roles of CD11/CD18 and ICAM-1 in acute Pseudomonas aeruginosa-induced pneumonia in mice. J Immunol 157, 5016-5021 (1996).

44. Ridger, V.C., Wagner, B.E., Wallace, W.A. \& Hellewell, P.G. Differential effects of CD18, CD29, and CD49 integrin subunit inhibition on neutrophil migration in pulmonary inflammation. J Immunol 166, 3484-3490 (2001).

45. Shang, X.Z. \& Issekutz, A.C. Beta 2 (CD18) and beta 1 (CD29) integrin mechanisms in migration of human polymorphonuclear leucocytes and monocytes through lung fibroblast barriers: shared and distinct mechanisms. Immunology 92, 527-535 (1997).

46. Shang, T., Yednock, T. \& Issekutz, A.C. alpha9beta1 integrin is expressed on human neutrophils and contributes to neutrophil migration through human lung and synovial fibroblast barriers. J Leukoc Biol 66, 809-816 (1999).

47. Burns, J.A., Issekutz, T.B., Yagita, H. \& Issekutz, A.C. The beta2, alpha4, alpha5 integrins and selectins mediate chemotactic factor and endotoxin enhanced neutrophil sequestration in the lung. Am J Pathol 158, 1809-1819 (2001).

48. Roman, J., Ritzenthaler, J.D., Boles, B., Lois, M. \& Roser-Page, S. Lipopolysaccharide induces expression of fibronectin alpha5 beta 1 integrin receptors in human monocytic cells in a protein kinase $\mathrm{C}$-dependent fashion. $\mathrm{Am} J$ Physiol Lung Cell Mol Physiol 287, L239-249 (2004).

49. Erzurum, S.C. et al. Mechanisms of lipopolysaccharide-induced neutrophil retention. Relative contributions of adhesive and cellular mechanical properties. $J$ Immunol 149, 154-162 (1992).

50. Sandström, T., Bjermer, L. \& Rylander, R. Lipopolysaccharide (LPS) inhalation in healthy subjects increases neutrophils, lymphocytes and fibronectin levels in bronchoalveolar lavage fluid. Eur Respir J 5, 992-996 (1992).

51. Pittet, J.F., Mackersie, R.C., Martin, T.R. \& Matthay, M.A. Biological markers of acute lung injury: prognostic and pathogenetic significance. Am J Respir Crit Care Med 155, 1187-1205 (1997). 
52. Kjæve, J., Næss, L., Ingebrigtsen, T., Vaage, J. \& Bjertnæs, L. Toxic oxygen metabolites increase microvascular permeability in isolated perfused rat lungs: The effect of methylprednisolone. Circ Shock L33, 228-232 (1991).

53. Gillissen, A. \& Nowak, D. Characterization of N-acetylcysteine and ambroxol in anti-oxidant therapy. Respir Med 92, 609-623 (1998).

54. Rosseau, S. et al. Monocyte migration through the alveolar epithelial barrier: Adhesion molecule mechanisms and impact of chemokines. J Immunol 164, 427435 (2000).

55. Maus, U.A. et al. Monocytes are potent facilitators of alveolar neutrophil emigration during lung inflammation: Role of the CCL2-CCR2 axis. J Immunol 170, 3273-3278 (2003).

56. Matute-Bello, G., Frevert, C.W. \& Martin, T.R. Animal models of acute lung injury. Am J Physiol Lung Cell Mol Physiol 295, L379-399 (2008).

57. Doyle, R.L., Szaflarski, N., Modin, G.W., Wiener-Kronish, J.P. \& Matthay, M.A. Identification of patients with acute lung injury: Predictors of mortality. Am J Respir Crit Care Med 152, 1818-1824 (1995).

58. Mendelson, $\mathrm{C}$. The aspiration of stomach contents into the lungs during obstetric anesthesia. Am J Obstet Gynecol 52, 191 (1946).

59. Howlett, C.E. et al. Inhaled nitric oxide protects against hyperoxia-induced apoptosis in rat lungs. Am J Physiol Lung Cell Mol Physiol 277, L596-605 (1999).

60. Lachmann, B., Robertson, B. \& Vogel, J. In vivo lung lavage as an experimental model of the respiratory distress syndrome. Acta Anaesthesiol Scand 24, 231-236 (1980).

61. Vlahakis, N.E., Schroeder, M.A., Limper, A.H. \& Hubmayr, R.D. Stretch induces cytokine release by alveolar epithelial cells in vitro. Am J Physiol Lung Cell Mol Physiol 277, L167-173 (1999).

62. Walley, K.R., Lukacs, N.W., Standiford, T.J., Strieter, R.M. \& Kunkel, S.L. Balance of inflammatory cytokines related to severity and mortality of murine sepsis. Infect Immun 64, 4733-4738 (1996).

63. Villar, J. et al. Induction of the heat shock response reduces mortality rate and organ damage in a sepsis-induced acute lung injury model. Crit Care Med 22, 914-921 (1994).

64. Kang, J.L. et al. Genistein prevents nuclear factor-kappa B activation and acute lung injury induced by lipopolysaccharide. Am J Respir Crit Care Med 164, 2206$2212(2001)$.

65. van Helden, H., Kuijpers, W., Steenvoorden, D., Go, C. \& Bruijnzeel, P. Intratracheal aerosolization of endotoxin (LPS) in the rat: A comprehensive animal model to study adult (acute) respiratory distress syndrome. Exp Lung Res 23, 297-316 (1997).

66. O'Grady, N.P. et al. Local inflammatory responses following bronchial endotoxin instillation in humans. Am J Respir Crit Care Med 163, 1591-1598 (2001).

67. Jaeschke, H., Farhood, A. \& Smith, C.W. Neutrophil-induced liver cell injury in endotoxin shock is a CD11b/CD18-dependent mechanism. Am J Physiol 261, G1051-1056 (1991). 
68. Wiener-Kronish, J.P. et al. Alveolar epithelial injury and pleural empyema in acute P. aeruginosa pneumonia in anesthetized rabbits. J Appl Physiol 75, 16611669 (1993).

69. Wang, H.L. et al. The intrinsic apoptotic pathway is required for lipopolysaccharide-induced lung endothelial cell death. J Immunol 179, 18341841 (2007).

70. Wiggs, B.R. et al. Contributions of capillary pathway size and neutrophil deformability to neutrophil transit through rabbit lungs. $J$ Appl Physiol 77, 463470 (1994).

71. Welty-Wolf, K.E. et al. Coagulation blockade prevents sepsis induced respiratory and renal failure in baboons. Am J Respir Crit Care Med 164, 1988-1996 (2001).

72. Suzuki, Y. et al. Effect of steroid on hyperoxia-induced ICAM-1 expression in pulmonary endothelial cells. Am J Physiol Lung Cell Mol Physiol 278, L245-252 (2000).

73. Brostjan, C., Anrather, J., Csizmadia, V., Natarajan, G. \& Winkler, H. Glucocorticoids inhibit E-selectin expression by targeting NF-kappaB and not ATF/c-Jun. J Immunol 158, 3836-3844 (1997).

74. Baeuerle, P.A. \& Baltimore, D. NF-kappa B: ten years after. Cell 87, 13-20 (1996).

75. Barnes, P.J. \& Karin, M. Nuclear factor-kappaB: a pivotal transcription factor in chronic inflammatory diseases. N Engl J Med 336, 1066-1071 (1997).

76. Bamberger, C.M., Schulte, H.M. \& Chrousos, G.P. Molecular determinants of glucocorticoid receptor function and tissue sensitivity to glucocorticoids. Endocr Rev 17, 245-261 (1996).

77. Wissink, S., van Heerde, E.C., vand der Burg, B. \& van der Saag, P.T. A dual mechanism mediates repression of NF-kappaB activity by glucocorticoids. Mol Endocrinol 12, 355-363 (1998).

78. Scheinman, R.I., Cogswell, P.C., Lofquist, A.K. \& Baldwin, A.S., Jr. Role of transcriptional activation of I kappa B alpha in mediation of immunosuppression by glucocorticoids. Science 270, 283-286 (1995).

79. Hofmann, T.G., Hehner, S.P., Bacher, S., Droge, W. \& Schmitz, M.L. Various glucocorticoids differ in their ability to induce gene expression, apoptosis and to repress NF-kappaB-dependent transcription. FEBS Lett 441, 441-446 (1998).

80. Sheppard, K.A. et al. Nuclear integration of glucocorticoid receptor and nuclear factor-kappaB signaling by CREB-binding protein and steroid receptor coactivator-1. J Biol Chem 273, 29291-29294 (1998).

81. Franchimont, D., Kino, T., Galon, J., Meduri, G.U. \& Chrousos, G. Glucocorticoids and inflammation revisited: The state of the art. NIH clinical staff conference. Neuroimmunomodulation 10, 247-260 (2002).

82. Miyamasu, M. et al. Glucocorticoids inhibit chemokine generation by human eosinophils. J Allergy Clin Immunol 101, 75-83 (1998).

83. Elovaara, I., Lalla, M., Spare, E., Lehtimaki, T. \& Dastidar, P. Methylprednisolone reduces adhesion molecules in blood and cerebrospinal fluid in patients with MS. Neurology 51, 1703-1708 (1998).

84. Cronstein, B.N., Kimmel, S.C., Levin, R.I., Martiniuk, F. \& Weissmann, G. A Mechanism for the antiinflammatory effects of corticosteroids: The glucocorticoid 
receptor regulates leukocyte adhesion to endothelial cells and expression of endothelial-leukocyte adhesion molecule 1 and intercellular adhesion molecule 1. Proc Natl Acad Sci U S A 89, 9991-9995 (1992).

85. Meduri, G.U. et al. Effect of prolonged methylprednisolone therapy in unresolving acute respiratory distress syndrome: A randomized controlled trial. JAMA 280, 159-165 (1998).

86. Filep, J.G., Delalandre, A., Payette, Y. \& Foldes-Filep, E. Glucocorticoid receptor regulates expression of L-selectin and CD11/CD18 on human neutrophils.

Circulation 96, 295-301 (1997).

87. Meduri, G.U. et al. Corticosteroid rescue treatment of progressive fibroproliferation in late ARDS. Patterns of response and predictors of outcome. Chest 105, 1516-1527 (1994).

88. Meduri, G.U. et al. Fibroproliferative phase of ARDS. Clinical findings and effects of corticosteroids. Chest 100, 943-952 (1991).

89. Meduri, G.U. \& Chrousos, G.P. Duration of glucocorticoid treatment and outcome in sepsis: Is the right drug used the wrong way? Chest 114, 355-360 (1998).

90. Holcroft, J.W., Vassar, M.J. \& Weber, C.J. Prostaglandin E1 and survival in patients with the adult respiratory distress syndrome. A prospective trial. Ann Surg 203, 371-378 (1986).

91. Bone, R.C. et al. Randomized double-blind, multicenter study of prostaglandin E1 in patients with the adult respiratory distress syndrome. Prostaglandin E1 Study Group. Chest 96, 114-119 (1989).

92. Abraham, E. et al. Liposomal prostaglandin E1 (TLC C-53) in acute respiratory distress syndrome: A controlled, randomized, double-blind, multicenter clinical trial. TLC C-53 ARDS Study Group. Crit Care Med 27, 1478-1485 (1999).

93. Ketoconazole for early treatment of acute lung injury and acute respiratory distress syndrome: A randomized controlled trial. The ARDS Network. JAMA 283, 1995-2002 (2000).

94. Welsh, C.H., Lien, D., Worthen, G.S. \& Weil, J.V. Pentoxifylline decreases endotoxin-induced pulmonary neutrophil sequestration and extravascular protein accumulation in the dog. Am Rev Respir Dis 138, 1106-1114 (1988).

95. Bursten, S.L. et al. Lisofylline causes rapid and prolonged suppression of serum levels of free fatty acids. J Pharmacol Exp Ther 284, 337-345 (1998).

96. Randomized, placebo-controlled trial of lisofylline for early treatment of acute lung injury and acute respiratory distress syndrome. Crit Care Med 30, 1-6 (2002).

97. Kietzmann, D., Kahl, R., Muller, M., Burchardi, H. \& Kettler, D. Hydrogen peroxide in expired breath condensate of patients with acute respiratory failure and with ARDS. Intensive Care Med 19, 78-81 (1993).

98. Bernard, G. N. Acetylcysteine in experimental and clinical acute lung injury. Am J Med 91, 54S-59S (1991).

99. Demling, R., LaLonde, C., Ikegami, K., Picard, L. \& Nayak, U. Alpha-tocopherol attenuates lung edema and lipid peroxidation caused by acute zymosan-induced peritonitis. Surgery 117, 226-231 (1995).

100. Wagner, P.D. et al. Protection against pulmonary $\mathrm{O}_{2}$ toxicity by $\mathrm{N}$-acetylcysteine. Eur Respir J 2, 116-126 (1989). 
101. Bernard, G.R. et al. A trial of antioxidants N-acetylcysteine and procysteine in ARDS. The antioxidant in ARDS study group. Chest 112, 164-172 (1997).

102. Meydani, S.N., Hayek, M. \& Coleman, L. Influence of vitamins E and B6 on immune response. Ann N Y Acad Sci 669, 125-139 (1992).

103. Folkesson, H.G., Matthay, M.A., Hebert, C.A. \& Broaddus, V.C. Acid aspiration induced lung injury in rabbits is mediated by interleukin-8-dependent mechanisms. J Clin Invest 96, 107-116 (1995).

104. Lewis, J. \& Jobe, A. Surfactant and the adult respiratory distress syndrome. Am Respir Dis 147, 218-233 (1993).

105. Gregory, T.J. et al. Surfactant chemical composition and biophysical activity in acute respiratory distress syndrome. J Clin Invest 88, 1976-1981 (1991).

106. Kesecioglu, J.Treatment of ALI/ARDS with surfactant. Am J Respir Crit Care Med 163, A819 (2001).

107. Anzueto, A. \& Melo, J. Acute respiratory distress syndrome: Liquid ventilation. Respir Care Clin N Am 4, 679-694 (1998).

108. Bernard, G.R. et al. Efficacy and safety of recombinant human activated protein C for severe sepsis. $N$ Engl J Med 344, 699-709 (2001).

109. Root, R.K. et al. Multicenter, double-blind, placebo-controlled study of the use of filgrastim in patients hospitalized with pneumonia and severe sepsis. Crit Care Med 31, 367-373 (2003).

110. McAuley, D.F., Frank, J.A., Fang, X. \& Matthay, M.A. Clinically relevant concentrations of beta 2 adrenergic agonists stimulate maximal cyclic adenosine monophosphate dependent airspace fluid clearance and decrease pulmonary edema in experimental acid induced lung injury. Crit Care Med 32, 1470-1476 (2004).

111. Lewis, C.A. \& Martin, G.S. Understanding and managing fluid balance in patients with acute lung injury. Curr Opin Crit Care 10, 13-17 (2004).

112. Gorman, S. \& Brown, P. Taming high-tech particles: Cautious steps into the nanotech future. The drug delivery companies report, 31-34 (2003).

113. Papahadjopoulos, D. \& Heath, T.Optimization of liposomes as carrier system for the intracellular delivery of the drugs and macromolecules. In: Liposomes and immunobiology, ed Baldwin, H.T. Elsevier, New York, 151-164 (1980).

114. Sapra, P. \& Allen, T. Ligand targeted liposomal anticancer drugs. Prog Lipid Res. Sep; 42, 439-462 (2003).

115. Zhou, R., Mazurchuk, R. \& Straubinger, R.M. Antivasculature effects of doxorubicin containing liposomes in an intracranial rat brain tumor model. Cancer Res 62, 2561-2566 (2002).

116. Chobanian, J.V., Tall, A.R. \& Brecher, P.I. Interaction between unilamellar egg yolk lecithin vesicles and human high density lipoprotein. Biochemistry 18, 180187 (1979).

117. Senior, J. \& Gregoriadis, G. Stability of small unilamellar liposomes in serum and clearance from the circulation: The effect of the phospholipid and cholesterol components. Life Sci 30, 2123-2136 (1982).

118. Semple, S.C., Chonn, A. \& Cullis, P.R. Influence of cholesterol on the association of plasma proteins with liposomes. Biochemistry 35, 2521-2525 (1996). 
119. Dos Santos, N. et al. Influence of poly(ethylene glycol) grafting density and polymer length on liposomes: Relating plasma circulation life times to protein binding. Biochim Biophys Acta 1768, 1367-1377 (2007).

120. Gabizon, A., Price, D.C., Huberty, J., Bresalier, R.S. \& Papahadjopoulos, D. Effect of liposome composition and other factors on the targeting of liposomes to experimental tumors: biodistribution and imaging studies. Cancer Res 50, 63716378 (1990).

121. Woodle, M.C., Newman, M.S. \& Cohen, J.A. Sterically stabilized liposomes: Physical and biological properties. J Drug Target 2, 397-403 (1994).

122. Drummond, D.C., Meyer, O., Hong, K., Kirpotin, D.B. \& Papahadjopoulos, D. Optimizing liposomes for delivery of chemotherapeutic agents to solid tumors. Pharmacol Rev 51, 691-743 (1999).

123. Allen, T., Hansen, C., Martin, F., Redemann, C. \& Yau-Young, A. Liposomes containing synthetic lipid derivatives of poly(ethylene glycol) show prolonged circulation half-lives in vivo. Biochim Biophys Acta. 1066, 29-36 (1991).

124. Needham, D. \& Nunn, R.S. Elastic deformation and failure of lipid bilayer membranes containing cholesterol. Biophys J 58, 997-1009 (1990).

125. Sankaram, M.B. \& Thompson, T.E. Interaction of cholesterol with various glycerophospholipids and sphingomyelin. Biochemistry 29, 10670-10675 (1990).

126. Veiga, M.P., Arrondo, J.L., Goni, F.M., Alonso, A. \& Marsh, D. Interaction of cholesterol with sphingomyelin in mixed membranes containing phosphatidylcholine, studied by spin-label ESR and IR spectroscopies. A possible stabilization of gel-phase sphingolipid domains by cholesterol. Biochemistry $\mathbf{4 0}$, 2614-2622 (2001).

127. Niemela, P., Hyvonen, M.T. \& Vattulainen, I. Structure and dynamics of sphingomyelin bilayer: Insight gained through systematic comparison to phosphatidylcholine. Biophys J 87, 2976-2989 (2004).

128. Johnston, M. et al. Characterization of the drug retention and pharmacokinetic properties of liposomal nanoparticles containing dihydrosphingomyelin. Biochim Biophys Acta 1768, 1121-1127 (2007).

129. Sharma, A. \& Straubinger, R.M. Novel taxol formulations: preparation and characterization of taxol-containing liposomes. Pharm Res 11, 889-896 (1994).

130. Chonn, A., Semple, S.C. \& Cullis, P.R. Association of blood proteins with large unilamellar liposomes in vivo. Relation to circulation life times. J Biol Chem 267, 18759-18765 (1992).

131. Yeeprae, W., Kawakami, S., Suzuki, S., Yamashita, F. \& Hashida, M. Physicochemical and pharmacokinetic characteristics of cationic liposomes. Pharmazie 61, 102-105 (2006).

132. Schmitt-Sody, M. et al. Neovascular targeting therapy: Paclitaxel encapsulated in cationic liposomes improves antitumoral efficacy. Clin Cancer Res 9, 2335-2341 (2003).

133. Blau, S., Jubeh, T.T., Haupt, S.M. \& Rubinstein, A. Drug targeting by surface cationization. Crit Rev Ther Drug Carrier Syst 17, 425-465 (2000).

134. Gabizon, A., Chemla, M., Tzemach, D., Horowitz, A.T. \& Goren, D. Liposome longevity and stability in circulation: Effects on the in vivo delivery to tumors and 
therapeutic efficacy of encapsulated anthracyclines. J Drug Target 3, 391-398 (1996).

135. JH, S. Fate and behaviour of liposomes in vivo: A review of controlling factors. CRC Crit Rev Ther Drug Carrier Syst 3, 123-193 (1987).

136. Kamps, J. \& Scherphof, G. Receptor versus non-receptor mediated clearance of liposomes. Adv Drug Deliv Rev 32, 81-97 (1998).

137. Gabizon, A., Barenholz, Y. \& Bialer, M. Prolongation of the circulation time of doxorubicin encapsulated in liposomes containing a polyethylene glycol derivatized phospholipid: Pharmacokinetic studies in rodents and dogs. Pharm Res 10, 703-708 (1993).

138. Li, X.M., Momsen, M.M., Smaby, J.M., Brockman, H.L. \& Brown, R.E. Cholesterol decreases the interfacial elasticity and detergent solubility of sphingomyelins. Biochemistry 40, 5954-5963 (2001).

139. Lande, M., Donovan, J. \& Zeidel, M. The relationship between membrane fluidity and permeabilities to water, solutes, ammonia, and protons. J Gen Physiol 106, 67-84 (1995).

140. Webb, M.S., Harasym, T.O., Masin, D., Bally, M.B. \& Mayer, L.D. Sphingomyelin-cholesterol liposomes significantly enhance the pharmacokinetic and therapeutic properties of vincristine in murine and human tumour models. $\mathrm{Br}$ J Cancer 72, 896-904 (1995).

141. Adlakha-Hutcheon, G., Bally, M.B., Shew, C.R. \& Madden, T.D. Controlled destabilization of a liposomal drug delivery system enhances mitoxantrone antitumor activity. Nat Biotechnol 17, 775-779 (1999).

142. Clerc, S. \& Barenholz, Y. Loading of amphipathic weak acids into liposomes in response to transmembrane calcium acetate gradients. Biochim Biophys Acta 1240, 257-265 (1995).

143. Fahr, A., van Hoogevest, P., Kuntsche, J. \& Leigh, M.L. Lipophilic drug transfer between liposomal and biological membranes: What does it mean for parenteral and oral drug delivery? J Liposome Res 16, 281-301 (2006).

144. Hayes, M.E. et al. Genospheres: self-assembling nucleic acid lipid nanoparticles suitable for targeted gene delivery. Gene Ther 13, 646-651 (2006).

145. Lasic, D. Doxorubicin in sterically stabilized liposomes. Nature April 11, 380, 561-562 (1996).

146. Woodle, M.C. et al. Versatility in lipid compositions showing prolonged circulation with sterically stabilized liposomes. Biochim Biophys Acta 1105, 193 200 (1992).

147. Allen, T.M. \& Chonn, A. Large unilamellar liposomes with low uptake into the reticuloendothelial system. FEBS Lett 223, 42-46 (1987).

148. Gabizon, A. \& Papahadjopoulos, D. Liposome formulations with prolonged circulation time in blood and enhanced uptake by tumors. Proc Natl Acad Sci US A 85, 6949-6953 (1988).

149. Torchilin, V.P., Shtilman, M.I., Trubetskoy, V.S., Whiteman, K. \& Milstein, A.M. Amphiphilic vinyl polymers effectively prolong liposome circulation time in vivo. Biochim Biophys Acta 1195, 181-184 (1994). 
150. Torchilin, V.P. et al. Poly(ethylene glycol) on the liposome surface: on the mechanism of polymer-coated liposome longevity. Biochim Biophys Acta 1195, 11-20 (1994).

151. Gabizon, A. \& Martin, F. Polyethylene glycol coated (pegylated) liposomal doxorubicin. Rationale for use in solid tumours. Drugs 54, 15-21 (1997).

152. Hashizume, H. et al. Openings between defective endothelial cells explain tumor vessel leakiness. Am J Pathol 156, 1363-1380 (2000).

153. Torchilin, V. Recent advances with liposomes as pharmaceutical carriers. Nat Rev Drug Discov. 4, 145-160 (2005).

154. Bangham, A.D. Liposomes: The Babraham connection. Chem Phys Lipids 64, 275-285 (1993).

155. Lasic, D.D. Novel applications of liposomes. Trends Biotechnol 16, 307-321 (1998).

156. Maruyama, K. et al. Targetability of novel immunoliposomes modified with amphipathic polyethylene glycols conjugated at their distal terminals to monoclonal antibodies. Biochim Biophys Acta 1234, 74-80 (1995).

157. Huwyler, J., Wu, D. \& Pardridge, W.M. Brain drug delivery of small molecules using immunoliposomes. Proc Natl Acad Sci U S A 93, 14164-14169 (1996).

158. Pagnan, G. et al. GD2-mediated melanoma cell targeting and cytotoxicity of liposome entrapped fenretinide. Int J Cancer 81, 268-274 (1999).

159. Blume, G. \& Cevc, G. Molecular mechanism of the lipid vesicle longevity in vivo. Biochim Biophys Acta 1146, 157-168 (1993).

160. Huang, A., Huang, L. \& Kennel, S.J. Monoclonal antibody covalently coupled with fatty acid. A reagent for in vitro liposome targeting. J Biol Chem 255, 80158018 (1980).

161. Weissig, V., Lasch, J., Klibanov, A.L. \& Torchilin, V.P. A new hydrophobic anchor for the attachment of proteins to liposomal membranes. FEBS Lett 202, 86-90 (1986).

162. Nobs, L., Buchegger, F., Gurny, R. \& Allémann, E. Attachment of homing moieties to colloidal carriers for active targeting: Comparison of different coupling methods, Edn. 2. (Marcel Dekker, New York).

163. Harsch, M., Walther, P. \& Weder, H.G. Targeting of monoclonal antibody coated liposomes to sheep red blood cells. Biochem Biophys Res Commun 103, 10691076 (1981).

164. Torchilin, V.P. et al. Incorporation of hydrophilic protein modified with hydrophobic agent into liposome membrane. Biochim Biophys Acta 602, 511-521 (1980).

165. Crosasso, P. et al. Antitumoral activity of liposomes and immunoliposomes containing 5-fluorouridine prodrugs. J Pharm Sci 86, 832-839 (1997).

166. Derksen, J. \& Scherphof, G. An improved method for the covalent coupling of proteins to liposomes. Biochim Biophys Acta 814, 151-155 (1985).

167. Derksen, J., Morselt, H. \& Scherphof, G. Uptake and processing of immunoglobulin coated liposomes by subpopulations of rat liver macrophages. Biochim Biophys Acta 971, 127-136 (1988).

168. Park, J.W. et al. Development of anti-p185HER2 immunoliposomes for cancer therapy. Proc Natl Acad Sci U S A 92, 1327-1331 (1995). 
169. Mercadal, M., Domingo, J., Petriz, J., Garcia, J. \& de Madariaga, M. A novel strategy affords high yield coupling of antibody to extremities of liposomal surface grafted PEG chains. Biochim Biophys Acta 1418, 232-238 (1999).

170. Hansen, C.B., Kao, G.Y., Moase, E.H., Zalipsky, S. \& Allen, T.M. Attachment of antibodies to sterically stabilized liposomes: Evaluation, comparison and optimization of coupling procedures. Biochim Biophys Acta 1239, 133-144 (1995).

171. Park, J.W. et al. Anti-HER2 immunoliposomes for targeted therapy of human tumors. Cancer Lett 118, 153-160 (1997).

172. Kirpotin, D. et al. Sterically stabilized anti-HER2 immunoliposomes: Design and targeting to human breast cancer cells in vitro. Biochemistry 36, 66-75 (1997).

173. Allen, T., Brandeis, E., Hansen, C., Kao, G. \& Zalipsky, S. A new strategy for attachment of antibodies to sterically stabilized liposomes resulting in efficient targeting to cancer cells. Biochim Biophys Acta 127, 99-108 (1995).

174. Martin, F.J., Hubbell, W.L. \& Papahadjopoulos, D. Immunospecific targeting of liposomes to cells: A novel and efficient method for covalent attachment of Fab' fragments via disulfide bonds. Biochemistry 20, 4229-4238 (1981).

175. Leserman, L., Barbet, J., Kourilsky, F. \& Weinstein, J. Targeting to cells of fluorescent liposomes covalently coupled with monoclonal antibody or protein. Nature 288, 602-604 (1980).

176. Ishida, O. et al. Liposomes bearing polyethyleneglycol coupled transferrin with intracellular targeting property to the solid tumors in vivo. Pharm Res 18, 10421048 (2001).

177. Maruyama, K. et al. Characterization of in vivo immunoliposome targeting to pulmonary endothelium. J Pharm Sci 79, 978-984 (1990).

178. Chua, M., Fan, S. \& Karush, F. Attachment of immunoglobulin to liposomal membrane via protein carbohydrate. Biochim Biophys Acta 800, 291-300 (1984).

179. Harding, J., Engbers, C., Newman, M., Goldstein, N. \& Zalipsky, S. Immunogenicity and pharmacokinetic attributes of poly(ethylene glycol) grafted immunoliposomes. Biochim Biophys Acta 1327, 181-192 (1997).

180. Zalipsky, S. Synthesis of an end-group functionalized polyethylene glycol-lipid conjugate for preparation of polymer grafted liposomes. Bioconjug Chem 4, 296299 (1993).

181. Torchilin, V., Goldmacher, V. \& Smirnov, V. Comparative studies on covalent and non-covalent immobilization of protein molecules on the surface of liposomes. Biochem Biophys Res Commun 85, 983-990 (1978).

182. Torchilin, V., Khaw, B., Smirnov, V. \& Haber, E. Preservation of antimyosin antibody activity after covalent coupling to liposomes. Biochem Biophys Res Commun 89, 1114-1119 (1979).

183. Kang, Y.S. \& Pardridge, W.M. Use of neutral avidin improves pharmacokinetics and brain delivery of biotin bound to an avidin-monoclonal antibody conjugate. $J$ Pharmacol Exp Ther 269, 344-350 (1994).

184. Schnyder, A., Krahenbuhl, S., Torok, M., Drewe, J. \& Huwyler, J. Targeting of skeletal muscle in vitro using biotinylated immunoliposomes. Biochem J 377, 6167 (2004). 
185. Loughrey, H., Bally, M. \& Cullis, P. A non-covalent method of attaching antibodies to liposomes. Biochim Biophys Acta 901, 157-160 (1987).

186. Mao, S.Y. Biotinylation of antibodies. Methods Mol Biol 34, 49-52 (1994).

187. Lasic, D.D., Frederik, P.M., Stuart, M.C., Barenholz, Y. \& McIntosh, T.J. Gelation of liposome interior. A novel method for drug encapsulation. FEBS Lett 312, 255 258 (1992).

188. Winterhalter, M. \& Lasic, D.D. Liposome stability and formation: Experimental parameters and theories on the size distribution. Chem Phys Lipids 64, 35-43 (1993).

189. Peleg-Shulman, T., Gibson, D., Cohen, R., Abra, R. \& Barenholz, Y. Characterization of sterically stabilized cisplatin liposomes by nuclear magnetic resonance. Biochim Biophys Acta 1510, 278-291 (2001).

190. Olson, F., Hunt, C.A., Szoka, F.C., Vail, W.J. \& Papahadjopoulos, D. Preparation of liposomes of defined size distribution by extrusion through polycarbonate membranes. Biochim Biophys Acta 557, 9-23 (1979).

191. Li, W. \& Szoka, F.C., Jr. Lipid based nanoparticles for nucleic acid delivery. Pharm Res 24, 438-449 (2007).

192. Mayer, L., Bally, M., Hope, M. \& Cullis, P. Uptake of antineoplastic agents into large unilamellar vesicles in response to a membrane potential. Biochim Biophys Acta 816, 294-302 (1985).

193. Cullis, P.R. et al. Influence of $\mathrm{pH}$ gradients on the transbilayer transport of drugs, lipids, peptides and metal ions into large unilamellar vesicles. Biochim Biophys Acta 1331, 187-211 (1997).

194. Allen, T.M., Newman, M.S., Woodle, M.C., Mayhew, E. \& Uster, P.S. Pharmacokinetics and anti-tumor activity of vincristine encapsulated in sterically stabilized liposomes. Int J Cancer 62, 199-204 (1995).

195. Haran, G., Cohen, R., Bar, L.K. \& Barenholz, Y. Transmembrane ammonium sulfate gradients in liposomes produce efficient and stable entrapment of amphipathic weak bases. Biochimica et Biophysica Acta - Biomembranes 1151, 201-215 (1993).

196. Lasic, D.D. et al. Transmembrane gradient driven phase transitions within vesicles: Lessons for drug delivery. Biochim Biophys Acta 1239, 145-156 (1995).

197. Drummond, D.C. et al. Development of a highly active nanoliposomal irinotecan using a novel intraliposomal stabilization strategy. Cancer Res 66, 3271-3277 (2006).

198. Ramsay, E. et al. Transition metal mediated liposomal encapsulation of irinotecan (CPT-11) stabilizes the drug in the therapeutically active lactone conformation. Pharm Res 23, 2799-2808 (2006).

199. Taggar, A.S. et al. Copper-topotecan complexation mediates drug accumulation into liposomes. J Control Release 114, 78-88 (2006).

200. Abraham, S.A. et al. An evaluation of transmembrane ion gradient mediated encapsulation of topotecan within liposomes. J Control Release 96, 449-461 (2004).

201. Kirpotin, D. Compound loaded liposomes and methods for their preparation. US PTO 6110491, 1-11 (2000). 
202. Fritze, A., Hens, F., Kimpfler, A., Schubert, R. \& Peschka-Suss, R. Remote loading of doxorubicin into liposomes driven by a transmembrane phosphate gradient. Biochim Biophys Acta 1758, 1633-1640 (2006).

203. Messerer, C.L. et al. Liposomal irinotecan: formulation development and therapeutic assessment in murine xenograft models of colorectal cancer. Clin Cancer Res 10, 6638-6649 (2004).

204. Zhigaltsev, I.V., Maurer, N., Edwards, K., Karlsson, G. \& Cullis, P.R. Formation of drug-arylsulfonate complexes inside liposomes: A novel approach to improve drug retention. J Control Release 110, 378-386 (2006).

205. Tardi, P.G. et al. Coencapsulation of irinotecan and floxuridine into low cholesterol containing liposomes that coordinate drug release in vivo. Biochim Biophys Acta 1768, 678-687 (2007).

206. Maurer-Spurej, E., Wong, K.F., Maurer, N., Fenske, D.B. \& Cullis, P.R. Factors influencing uptake and retention of amino-containing drugs in large unilamellar vesicles exhibiting transmembrane $\mathrm{pH}$ gradients. Biochim Biophys Acta 1416, 110 (1999).

207. Webb, M.S. et al. Comparison of different hydrophobic anchors conjugated to poly(ethylene glycol): Effects on the pharmacokinetics of liposomal vincristine. Biochim Biophys Acta 1372, 272-282 (1998).

208. Mayer, L.D. \& St-Onge, G. Determination of free and liposome associated doxorubicin and vincristine levels in plasma under equilibrium conditions employing ultrafiltration techniques. Anal Biochem 232, 149-157 (1995).

209. Sparreboom, A. et al. Comparative preclinical and clinical pharmacokinetics of a cremophor-free, nanoparticle albumin-bound paclitaxel (ABI-007) and paclitaxel formulated in Cremophor (Taxol). Clin Cancer Res 11, 4136-4143 (2005).

210. Mross, K. et al. Pharmacokinetics of liposomal doxorubicin (TLC-D99; Myocet) in patients with solid tumors: An open-label, single dose study. Cancer Chemother Pharmacol 54, 514-524 (2004).

211. Manochakian, R., Miller, K.C. \& Chanan-Khan, A.A. Bortezomib in combination with pegylated liposomal doxorubicin for the treatment of multiple myeloma. Clin Lymphoma Myeloma 7, 266-271 (2007).

212. Koshkina, N.V., Golunski, E., Roberts, L.E., Gilbert, B.E. \& Knight, V. Cyclosporin A aerosol improves the anticancer effect of paclitaxel aerosol in mice. J Aerosol Med 17, 7-14 (2004).

213. Lo, Y.L., Tsai, J.C. \& Kuo, J.H. Liposomes and disaccharides as carriers in spraydried powder formulations of superoxide dismutase. $J$ Control Release 94, 259272 (2004).

214. Vyas, S.P., Kannan, M.E., Jain, S., Mishra, V. \& Singh, P. Design of liposomal aerosols for improved delivery of rifampicin to alveolar macrophages. Int $J$ Pharm 269, 37-49 (2004).

215. Konduri, K.S. et al. Efficacy of liposomal budesonide in experimental asthma. $J$ Allergy Clin Immunol 111, 321-327 (2003).

216. Gilbert, B.E., Seryshev, A., Knight, V. \& Brayton, C. 9-nitrocamptothecin liposome aerosol: lack of subacute toxicity in dogs. Inhal Toxicol 14, 185-197 (2002). 
217. Koshkina, N.V. et al. 9-Nitrocamptothecin liposome aerosol treatment of melanoma and osteosarcoma lung metastases in mice. Clin Cancer Res 6, 28762880 (2000).

218. Desai, T.R., Hancock, R.E. \& Finlay, W.H. A facile method of delivery of liposomes by nebulization. J Control Release 84, 69-78 (2002).

219. Heurtault, B., Saulnier, P., Pech, B., Proust, J.E. \& Benoit, J.P. A novel phase inversion based process for the preparation of lipid nanocarriers. Pharm Res 19, 875-880 (2002).

220. Heurtault, B., Saulnier, P., Pech, B., Proust, J.E. \& Benoit, J.P. Physico-chemical stability of colloidal lipid particles. Biomaterials 24, 4283-4300 (2003).

221. Smoluchowski, M. Zur kinetischen theorie der brownschen molekularbewegung und der suspensionen. Annalen der Physik, 21 756-780 (1906).

222. Gouy, G.L. Sur la constitution de la charge électrique a la surface d'un électrolyte J Phys 9, 457 (1910).

223. Jones, M.N. The surface properties of phospholipid liposome systems and their characterisation. Adv Colloid Interface Sci 54, 93-128 (1995).

224. Chapman, D. A contribution to the theory of electrocapillarity. Philos Mag 25, 475 (1913).

225. Derjaguin, B.V., Rabinovich, Y.I., Churaev, N.V. Direct measurement of molecular forces Nature Reviews 272, 313-318 (1978).

226. Hunter, R.J Particle size and zeta potential and other parameters of the model dispersed phase. In: Zeta potential in colloid science, ed Hunter, R.J. Academic press, London, 1-33 (1981).

227. Boroske, E. \& Helfrich, W. Magnetic anisotropy of egg lecithin membranes. Biophys J 24, 863-868 (1978).

228. Israelachvili, J.N. \& McGuiggan, P.M. Forces between surfaces in liquids. Science 241, 795-800 (1988).

229. de Gennes, P.G. Polymers at an interface; A simplified view. Advances in Colloid and Interface Science 27, 189-209 (1987).

230. Dolan, A. \& Edwards, S. Theory of the stabilization of colloids by adsorbed polymer. Proceedings of the royal society of London. Series A, mathematical and physical sciences 337, 509-516 (1974).

231. Hristova, K. \& Needham, D. The influence of polymer grafted lipids on the physical properties of lipid bilayers: A theoretical study. Journal of Colloid and Interface Science 168, 302-314 (1994).

232. Klein, J. The interdiffision of polymers. Science 250, 640-646 (1990).

233. Kenworthy, A.K., Hristova, K., Needham, D. \& McIntosh, T.J. Range and magnitude of the steric pressure between bilayers containing phospholipids with covalently attached poly(ethylene glycol). Biophys $J$ 68, 1921-1936 (1995).

234. Kenworthy, A.K., Simon, S.A. \& McIntosh, T.J. Structure and phase behavior of lipid suspensions containing phospholipids with covalently attached poly(ethylene glycol). Biophys J 68, 1903-1920 (1995).

235. Needham, D., McIntosh, T.J. \& Lasic, D.D. Repulsive interactions and mechanical stability of polymer grafted lipid membranes. Biochim Biophys Acta 1108, 40-48 (1992). 
236. Smoluchowski, M. Drei vorträge über diffusion, brownsche molekularbewegung und koagulation von kolloidteilchen Phys Z 17, 557-571 (1916).

237. Smoluchowski, M. Versuch einer mathematischen theorie der koagulationskinetik kolloider lösungen Z Phys Chem 92, 129-168 (1917).

238. Epstein, H. et al. Number concentration of nanoparticles in liposomal and polymeric multiparticulate preparations: empirical and calculation methods. Biomaterials 27, 651-659 (2006).

239. Jennings, T.A. The primary drying process. In: Lyophilization: Introduction and basic principles, ed Jennings, T.A. Interpharm press, Engelwood, CO, 283-354 (1990).

240. Gea, G.H. Thermodynamic lyophilization control-TLC. Process Analytical Technology 12, 1-9 (2008).

241. Her, L.M. \& Nail, S.L. Measurement of glass transition temperatures of freezeconcentrated solutes by differential scanning calorimetry. Pharm Res 11, 54-59 (1994).

242. van Winden, E.C., Talsma, H. \& Crommelin, D.J. Thermal analysis of freezedried liposome-carbohydrate mixtures with modulated temperature differential scanning calorimetry. J Pharm Sci 87, 231-237 (1998).

243. Searles, J.A., Carpenter, J.F. \& Randolph, T.W. Annealing to optimize the primary drying rate, reduce freezing-induced drying rate heterogeneity, and determine $\mathrm{T}(\mathrm{g})$ ' in pharmaceutical lyophilization. J Pharm Sci 90, 872-887 (2001).

244. van Winden, E.C., Zhang, W. \& Crommelin, D.J. Effect of freezing rate on the stability of liposomes during freeze-drying and rehydration. Pharm Res 14, 11511160 (1997).

245. Ybema, H., Kolkman-Roodbeen, L., te Booy, M.P. \& Vromans, H. Vial lyophilization: Calculations on rate limitation during primary drying. Pharm Res 12, 1260-1263 (1995).

246. Crowe, J.M \& Crowe, L.M Preservation of liposomes by freeze-drying. In: Liposome technology: Entrapment of drugs and other materials, ed Gregoriadis, G. CRC Press, Boca Raton, FL, 229-252 (1993).

247. Crowe, J.H., Leslie, S.B. \& Crowe, L.M. Is vitrification sufficient to preserve liposomes during freeze-drying? Cryobiology 31, 355-366 (1994).

248. Wolfe, J. \& Bryant, G. Freezing, drying, and/or vitrification of membrane- solutewater systems. Cryobiology 39, 103-129 (1999).

249. Koster, K.L., Lei, Y.P., Anderson, M., Martin, S. \& Bryant, G. Effects of vitrified and non-vitrified sugars on phosphatidylcholine fluid-to-gel phase transitions. Biophys J 78, 1932-1946 (2000).

250. Crowe, J.H. et al. Interactions of sugars with membranes. Biochim Biophys Acta 947, 367-384 (1988).

251. van Winden Ewoud, C.A. Freeze-drying of liposomes: Theory and practice. Methods in Enzymology 367, 99-110 (2003).

252. Jungalwala, F.B., Turel, R.J., Evans, J.E. \& McCluer, R.H. Sensitive analysis of ethanolamine- and serine-containing phosphoglycerides by high performance liquid chromatography. Biochem J 145, 517-526 (1975). 
253. Gokhale, P.C. et al. Improved safety, pharmacokinetics and therapeutic efficacy profiles of a novel liposomal formulation of mitoxantrone. Anticancer Res 21, 3313-3321 (2001).

254. Zhang, J.A. et al. Development and characterization of a novel liposome based formulation of SN-38. Int J Pharm 270, 93-107 (2004).

255. Carroll, R. \& Rudel, L. Evaluation of a high-performance liquid chromatography method for isolation and quantitation of cholesterol and cholesteryl esters. J. Lipid Res. 22, 359-363 (1981).

256. Hamilton, J. \& Comai, K. Separation of neutral lipids and free fatty acids by high performance liquid chromatography using low wavelength ultraviolet detection. $J$. Lipid Res. 25, 1142-1148 (1984).

257. Hax, W.M. \& van Kessel, W.S. High performance liquid chromatographic separation and photometric detection of phospholipids. J Chromatogr 142, 735741 (1977).

258. Van Kessel, W.S., Hax, W.M., Demel, R.A. \& De Gier, J. High performance liquid chromatographic separation and direct ultraviolet detection of phospholipids. Biochim Biophys Acta 486, 524-530 (1977).

259. Meyer, O., Roch, O., Elmlinger, D. \& Kolbe, H.V. Direct lipid quantitation of cationic liposomes by reversed-phase HPLC in lipoplex preparation process. Eur J Pharm Biopharm 50, 353-356 (2000).

260. Lang, J.K. Quantitative determination of cholesterol in liposome drug products and raw materials by high performance liquid chromatography. J Chromatogr 507, 157-163 (1990).

261. Chow, C.Y. \& Heath, T.D. Rapid diffusion of the lipid phosphorus of phosphatidylglycerol liposomes through polycarbonate membranes is caused by the oxidation of the unsaturated fatty acids. Biochim Biophys Acta 1239, 168-176 (1995).

262. Shimizu, Y., Nakata, M., Matsunuma, J. \& Mizuochi, T. Simultaneous quantification of components of neoglycolipid-coated liposomes using high performance liquid chromatography with evaporative light scattering detection. $J$ Chromatogr B Biomed Sci Appl 754, 127-133 (2001).

263. ICH. Q2B validation of analytical procedures: Methodology, international conference on harmonization of technical requirements for registration of pharmaceutical for human use. ICH guidelines, 1-12 (1997).

264. ICH. Reviewer guidance: Validation of chromatographic methods, US department of health and human services, US food and drug administration, center for drug evaluation and research. ICH guidelines, 1-33 (1994).

265. Meduri, G.U. \& Kanangat, S. Glucocorticoid treatment of sepsis and acute respiratory distress syndrome: time for a critical reappraisal. Crit Care Med 26, 630-633 (1998).

266. Ellman, G.L. Tissue sulfhydryl groups. Arch Biochem Biophys 82, $70-77$ (1959).

267. Iden, D.L. \& Allen, T.M. In vitro and in vivo comparison of immunoliposomes made by conventional coupling techniques with those made by a new postinsertion approach. Biochim Biophys Acta 1513, 207-216 (2001). 
268. Saul, J.M., Annapragada, A., Natarajan, J.V. \& Bellamkonda, R.V. Controlled targeting of liposomal doxorubicin via the folate receptor in vitro. J Control Release 92, 49-67 (2003).

269. Mayer, L.D., Hope, M.J. \& Cullis, P.R. Vesicles of variable sizes produced by a rapid extrusion procedure. Biochim Biophys Acta 858, 161-168 (1986).

270. Eastman, S.J., Wilschut, J., Cullis, P.R. \& Hope, M.J. Intervesicular exchange of lipids with weak acid and weak base characteristics: Influence of transmembrane pH gradients. Biochim Biophys Acta 981, 178-184 (1989).

271. Kolchens, S., Ramaswami, V., Birgenheier, J., Nett, L. \& O'Brien, D.F. Quasielastic light scattering determination of the size distribution of extruded vesicles. Chem Phys Lipids 65, 1-10 (1993).

272. Costa, P. \& Sousa Lobo, J.M. Modeling and comparison of dissolution profiles. Eur J Pharm Sci 13, 123-133 (2001).

273. Higuchi, T. Rate of release of medicaments from ointment bases containing drugs in suspension. J Pharm Sci 50, 874-875 (1961).

274. Goldberg, A.H., Higuchi, W.I., Ho, N.F. \& Zografi, G. Mechanisms of interphase transport. I. Theoretical considerations of diffusion and interfacial barriers in transport of solubilized systems. J Pharm Sci 56, 1432-1437 (1967).

275. Lapidus, H. \& Lordi, N.G. Drug release from compressed hydrophilic matrices. $J$ Pharm Sci 57, 1292-1301 (1968).

276. Langer, R. \& Peppas, N. Advances in biomaterials, drug delivery, and bionanotechnology. AICHE 49, 2990-3006 (2003).

277. Narasimhan, B., Mallapragada, S. \& Peppas, N. Release kinetics-data interpretation. In: Encyclopedia of controlled drug delivery, ed Mathiwitz, E. John Wiley, New York, 921-935 (1999).

278. Chen, H. \& Langer, R. Magnetically responsive polymerized liposomes as potential oral delivery vehicles. Pharm Res 14, 537-540 (1997).

279. Silvander, M., Hansson, P. \& Edwards, K. Liposomal surface potential and bilayer packing as affected by PEG-lipid inclusion. Langmuir 8, 3696-3702 (2000).

280. Szoka, F. \& Tang, M. Amphotericin B formulated in liposomes and lipid based systems: A review. J. Liposome Res 3, 363-375 (1993).

281. Villivalam, V., Rege, P. \& Collins, C. Development in release testing of topical dosage forms: Use of the enhancer cell TM with automated sampling. J Pharm Biomed Anal. 17, 1225-1233 (1998).

282. Romero-Cano, M., Martín-Rodríguez, A. \& de las Nieves, F. Electrosteric stabilization of polymer colloids with different functionality. Langmuir 17, 35053511 (2001).

283. Bisby, R.H., Mead, C., Mitchell, A.C. \& Morgan, C.G. Fast laser-induced solute release from liposomes sensitized with photochromic lipid: Effects of temperature, lipid host, and sensitizer concentration. Biochemical and Biophysical Research Communications 262, 406-410 (1999).

284. Bolotin, E.M. et al. Ammonium sulfate gradients for efficient and stable remote loading of amphipathic weak bases into liposomes and ligandoliposomes. Journal of Liposome Research 4, 455-479 (1994). 
285. Stubbs, M., McSheehy, P.M., Griffiths, J.R. \& Bashford, C.L. Causes and consequences of tumour acidity and implications for treatment. Mol Med Today $\mathbf{6}$, 15-19 (2000).

286. Hafez, I.M., Ansell, S. \& Cullis, P.R. Tunable pH sensitive liposomes composed of mixtures of cationic and anionic lipids. Biophysical Journal 79, 1438-1446 (2000).

287. Hofmeister, F. Zur lehre von der wirkung der salze. zweite mitteilung. Arch Exp Pathol Pharmakol 24, 247-260 (1888).

288. Bowron, D.T. \& Finney, J.L. Structure of a salt--amphiphile--water solution and the mechanism of salting out. $J$ Chem Phys 118, 8357-8372 (2003).

289. Gregoriadis, G. Drug entrapment in liposomes. FEBS Lett 36, 292-296 (1973).

290. Perrie, Y. \& Gregoriadis, G. Liposome entrapped plasmid DNA: characterization studies. Biochim Biophys Acta 1475, 125-132 (2000).

291. McMullen, T.P., Lewis, R.N. \& McElhaney, R.N. Differential scanning calorimetric study of the effect of cholesterol on the thermotropic phase behavior of a homologous series of linear saturated phosphatidylcholines. Biochemistry 32, 516-522 (1993).

292. Komatsu, H. et al. Effects of the acyl chain composition of phosphatidylcholines on the stability of freeze-dried small liposomes in the presence of maltose. Chem Phys Lipids 113, 29-39 (2001).

293. Wolkers, W.F., Oldenhof, H., Tablin, F. \& Crowe, J.H. Preservation of dried liposomes in the presence of sugar and phosphate. Biochim Biophys Acta 1661, 125-134 (2004).

294. Anchordoguy, T., Carpenter, J.F., Loomis, S.H. \& Crowe, J.H. Mechanisms of interaction of amino acids with phospholipid bilayers during freezing. Biochim Biophys Acta 946, 299-306 (1988).

295. Crowe, J.H. \& Crowe, L.M. Factors affecting the stability of dry liposomes. Biochim Biophys Acta 939, 327-334 (1988).

296. Kirby, C.J. \& Gregoriadis, G. Preparation of liposomes containing factor VIII for oral treatment of haemophilia. J Microencapsul 1, 33-45 (1984).

297. Crowe, L.M., Crowe, J.H., Rudolph, A., Womersley, C. \& Appel, L. Preservation of freeze-dried liposomes by trehalose. Arch Biochem Biophys 242, 240-247 (1985).

298. Crowe, L.M., Crowe, J.H. \& Chapman, D. Interaction of carbohydrates with dry dipalmitoylphosphatidylcholine. Arch Biochem Biophys 236, 289-296 (1985).

299. Crowe, J., Clegg, J. \& Crowe, L. Anhydrobiosis: The water replacement hypothesis.In: The properties of water in foods (ISOPOW 6), ed Reid, D.S. Chapman \& Hall, New York, 440-455 (1998).

300. Koster, K.L., Webb, M.S., Bryant, G. \& Lynch, D.V. Interactions between soluble sugars and POPC (1-palmitoyl-2-oleoylphosphatidylcholine) during dehydration: vitrification of sugars alters the phase behavior of the phospholipid. Biochim Biophys Acta 1193, 143-150 (1994).

301. Leslie, S.B., Israeli, E., Lighthart, B., Crowe, J.H. \& Crowe, L.M. Trehalose and sucrose protect both membranes and proteins in intact bacteria during drying. Appl Environ Microbiol 61, 3592-3597 (1995). 
302. Connors, K.A. The Karl Fisher titration of water. Drug Dev Ind Pharm. 14, 1891 1903 (1988).

303. Luzardo, M.C. et al. Effect of trehalose and sucrose on the hydration and dipole potential of lipid bilayers. Biophys J 78, 2452-2458 (2000).

304. Ohtake, S., Schebor, C., Palecek, S.P. \& de Pablo, J.J. Phase behavior of freezedried phospholipid-cholesterol mixtures stabilized with trehalose. Biochim Biophys Acta 1713, 57-64 (2005).

305. Koynova, R., Brankov, J. \& Tenchov, B. Modulation of lipid phase behavior by kosmotropic and chaotropic solutes. Experiment and thermodynamic theory. Eur Biophys J. 25, 261-274 (1997).

306. Crowe, J.H., Carpenter, J.F. \& Crowe, L.M. The role of vitrification in anhydrobiosis. Annu Rev Physiol 60, 73-103 (1998).

307. Crowe, J.H., Hoekstra, F.A., Nguyen, K.H. \& Crowe, L.M. Is vitrification involved in depression of the phase transition temperature in dry phospholipids? Biochim Biophys Acta 1280, 187-196 (1996).

308. Crowe, J.H. et al. The trehalose myth revisited: Introduction to a symposium on stabilization of cells in the dry state. Cryobiology 43, 89-105 (2001).

309. Nagase, H., Ueda, H. \& Nakagaki, M. Effect of water on lamellar structure of DPPC/sugar systems. Biochim Biophys Acta 1328, 197-206 (1997).

310. Sola-Penna, M. \& Meyer-Fernandes, J. Stabilization against thermal inactivation promoted by sugars on enzyme structure and function: Why is trehalose more effective than other sugars? Arch Biochem Biophys 360, 10-14 (1998).

311. Magazu, S., Migliardo, F., Mondelli, C. \& Vadala, M. Correlation between bioprotective effectiveness and dynamic properties of trehalose-water, maltosewater and sucrose-water mixtures. Carbohydr Res 340, 2796-2801 (2005).

312. Crowe, L.M., Reid, D.S. \& Crowe, J.H. Is trehalose special for preserving dry biomaterials? Biophys J 71, 2087-2093 (1996).

313. Suzuki, T., Komatsu, H. \& Miyajima, K. Effects of glucose and its oligomers on the stability of freeze-dried liposomes. Biochim Biophys Acta 1278, 176-182 (1996).

314. Kundu, C.N., Das, K. \& Majumder, G.C. Effect of amino acids on goat cauda epididymal sperm cryopreservation using a chemically defined model system. Cryobiology 42, 21-27 (2001).

315. Rubenfeld, G.D. \& Herridge, M.S. Epidemiology and outcomes of acute lung injury. Chest 131, 554-562 (2007).

316. Frank, J. et al. Transforming growth factor-beta1 decreases expression of the epithelial sodium channel alphaENaC and alveolar epithelial vectorial sodium and fluid transport via an ERK1/2-dependent mechanism. J Biol Chem 278, 4393943950 (2003).

317. Olman, M.A. et al. Microarray analysis indicates that pulmonary edema fluid from patients with acute lung injury mediates inflammation, mitogen gene expression, and fibroblast proliferation through bioactive interleukin-1. Chest 121, 69S-70S (2002).

318. Su, G. et al. Integrin \{alpha\} vbeta5 regulates lung vascular permeability and pulmonary endothelial barrier function. Am J Respir Cell Mol Biol 36, 377-386 (2007). 
319. Leff, J.A. et al. Interleukin-1-induced lung neutrophil accumulation and oxygen metabolite mediated lung leak in rats. Am J Physiol 266, L2-8 (1994).

320. Newton, R. et al. Repression of inflammatory gene expression in human pulmonary epithelial cells by small molecule IkappaB kinase inhibitors. $J$ Pharmacol Exp Ther 321, 734-742 (2007).

321. Goodman, R.B., Pugin, J., Lee, J.S. \& Matthay, M.A. Cytokine mediated inflammation in acute lung injury. Cytokine Growth Factor Rev 14, 523-535 (2003).

322. Lin, C.C. et al. Transactivation of Src, PDGF receptor, and Akt is involved in IL1beta-induced ICAM-1 expression in A549 cells. J Cell Physiol 211, 771-780 (2007).

323. Karin, M., Yamamoto, Y. \& Wang, Q.M. The IKK NF-kappa B system: A treasure trove for drug development. Nat Rev Drug Discov 3, 17-26 (2004).

324. Baldwin, A.S., Jr. The NF-kappa B and I kappa B proteins: New discoveries and insights. Annu Rev Immunol 14, 649-683 (1996).

325. Beg, A.A. \& Baldwin, A.S., Jr. The I kappa B proteins: multifunctional regulators of Rel/NF-kappa B transcription factors. Genes Dev 7, 2064-2070 (1993).

326. Sadikot, R. et al. Selective IkB Kinase Expression in airway epithelium generates neutrophilic lung inflammation. Journal of Immunology 170, 1091-1098 (2003).

327. Blackwell, T.S., Blackwell, T.R. \& Christman, J.W. Impaired activation of nuclear factor-kappaB in endotoxin tolerant rats is associated with down regulation of chemokine gene expression and inhibition of neutrophilic lung inflammation. $J$ Immunol 158, 5934-5940 (1997).

328. Newton, R. et al. Effect of dexamethasone on interleukin-1beta (IL-1beta)induced nuclear factor-kappaB (NF-kappaB) and kappaB-dependent transcription in epithelial cells. Eur J Biochem 254, 81-89 (1998).

329. Schneider, T. \& Issekutz, A. Quantitation of eosinophil and neutrophil infiltration into rat lung by specific assays for eosinophil peroxidase and myeloperoxidase: application in a Brown Norway rat model of allergic pulmonary inflammation. $J$. Immunol Methods 198 (1996).

330. Atabai, K. et al. Keratinocyte growth factor can enhance alveolar epithelial repair by nonmitogenic mechanisms. Am J Physiol Lung Cell Mol Physiol 283, L163169 (2002).

331. Hudson, L.D., Milberg, J.A., Anardi, D. \& Maunder, R.J. Clinical risks for development of the acute respiratory distress syndrome. Am J Respir Crit Care Med 151, 293-301 (1995).

332. Gonzalez-Amaro, R. \& Sanchez-Madrid, F. Cell adhesion molecules: Selectins and integrins. Crit Rev Immunol 19, 389-429 (1999).

333. Springer, T.A. Traffic signals on endothelium for lymphocyte recirculation and leukocyte emigration: The multistep paradigm. Cell 76, 301-314 (1995).

334. Roman, J. Extracellular matrix and lung inflammation. Immunol Res 15, 163-178 (1996).

335. Tsai, L.H. Stuck on the ECM. Trends Cell Biol 8, 292-295 (1998).

336. Abdul, B. et al. ICAM-1 and LFA play critical roles in LPS-induced neutrophil recruitment into the alveolar space. Am J Physiol Lung Cell Mol Physiol 291, L200-L207 (2006). 
337. Dustin, M.L. \& Springer, T.A. Lymphocyte function associated antigen-1 (LFA-1) interaction with intercellular adhesion molecule-1 (ICAM-1) is one of at least three mechanisms for lymphocyte adhesion to cultured endothelial cells. $J$ Cell Biol 107, 321-331 (1988).

338. Kumasaka, T. et al. Role of the intercellular adhesion molecule-1 (ICAM-1) in endotoxin-induced pneumonia evaluated using ICAM-1 antisense oligonucleotides, anti-ICAM-1 monoclonal antibodies, and ICAM-1 mutant mice. J Clin Invest 97, 2362-2369 (1996). 


\section{VITA}

Mr. Hari R. Desu son of Mr. Narayana Desu and Mrs. Lakshmi Desu was born in Narasaraopet, Andhra Pradesh, India. He was admitted to the College of Pharmacy, Jawaharlal Nehru Technology University (JNTU), Hyderabad, India. Mr. Hari Desu graduated with a Bachelor of Pharmacy degree with distinction. He received a Master of Science in Pharmaceutics from National Institute of Pharmaceutical Education and Research (NIPER), Chandigarh, India. Mr. Desu joined as a formulation research scientist in Dr. Reddy's Laboratories Ltd, Hyderabad, India.

Mr. Hari Desu was enrolled in the Doctor of Philosophy program at the University of Tennessee Health Science Center in August 2003 and received a Graduate Teaching Assistantship. At the same time, he joined Parenteral Medications Laboratories with Dr. George C. Wood as major advisor. During this period, he presented research work on novel parenteral formulations at various international conferences. He published the research work in various international journals. After graduation, he plans to pursue his research interests in formulation development sciences. Mr. Hari Desu is a member of the American Association of Pharmaceutical Scientists, Parenteral Drug Association and the Controlled Release Society. He was inducted into Rho Chi Society in 2006 for his accomplishments in academic carrier. Because of the unique and novel nature of the research work in $\mathrm{PhD}$ dissertation, Hari drafted two provisional patents. 